University of Rhode Island

DigitalCommons@URI

Open Access Master's Theses

2020

\title{
APPROXIMATE INVERSION OF MIMO SYSTEMS FOR PRECISION TRACKING
}

Roland G. Schurig

University of Rhode Island, roland.schurig@gmx.de

Follow this and additional works at: https://digitalcommons.uri.edu/theses

\section{Recommended Citation}

Schurig, Roland G., "APPROXIMATE INVERSION OF MIMO SYSTEMS FOR PRECISION TRACKING" (2020). Open Access Master's Theses. Paper 1871.

https://digitalcommons.uri.edu/theses/1871

This Thesis is brought to you for free and open access by DigitalCommons@URI. It has been accepted for inclusion in Open Access Master's Theses by an authorized administrator of DigitalCommons@URI. For more information, please contact digitalcommons-group@uri.edu. 


\section{APPROXIMATE INVERSION OF MIMO SYSTEMS \\ FOR PRECISION TRACKING \\ BY \\ ROLAND G. SCHURIG}

A THESIS SUBMITTED IN PARTIAL FULFILLMENT OF THE

REQUIREMENTS FOR THE DEGREE OF

MASTER OF SCIENCE

IN

ELECTRICAL ENGINEERING

UNIVERSITY OF RHODE ISLAND

2020 
MASTER OF SCIENCE THESIS

$\mathrm{OF}$

ROLAND G. SCHURIG

\title{
APPROVED:
}

Thesis Committee:

\author{
Major Professor Richard J. Vaccaro \\ Paolo Stegagno \\ Chengzhi Yuan \\ Brenton DeBoef \\ DEAN OF THE GRADUATE SCHOOL
}

\section{UNIVERSITY OF RHODE ISLAND}




\begin{abstract}
The problem considered is the design of a digital control system for precision tracking control of a fully-coupled non-minimum phase MIMO plant. The first step is to design a 2-degree-of-freedom feedback tracking system using standard pole-placement or linear quadratic regulator techniques. The result is a stable closed-loop system having zero steady-state error to step inputs. In order to obtain precision tracking for other types of inputs, some kind of feedforward control is needed. Two different tracking architectures are considered in this thesis, both feature a feedforward inverse filter to enable precision tracking. The filters are the inverse of a closed-loop system. The derivations of the filters are given; for non-minimum phase systems the result is approximately a decoupled system of delays over a certain bandwidth. Two different possibilities to design the inverse filters are considered: the first one is based on a novel frequency approximation, while the other one relies on the addition of feedback. Several options to design the feedback are discussed. The tracking performances of the resulting precision tracking architectures are demonstrated and evaluated for several non-minimum phase example systems via simulations.
\end{abstract}




\section{ACKNOWLEDGMENTS}

First and foremost, I would like to thank my major professor, Dr. Richard J. Vaccaro. His expertise was extremely helpful and priceless in every single meeting, in which we always made remarkable progress and I consistently left with new ideas to try out. Especially for me, as an international student, this very close cooperation with a professor was a new and unique experience, and I am truly grateful for Dr. Vaccaro's support.

I want to thank my committee members as well; Dr. Paolo Stegagno and Dr. Chengzhi Yuan, for their time, enriching remarks and helpful questions, and my Defense Chair; Dr. David Taggart, for a pleasant and well-organized oral examination.

Last but not least, I want to thank my family since, without their support, I may have never dared to spend a year abroad and without whom this dual degree would have not been possible. 


\section{TABLE OF CONTENTS}

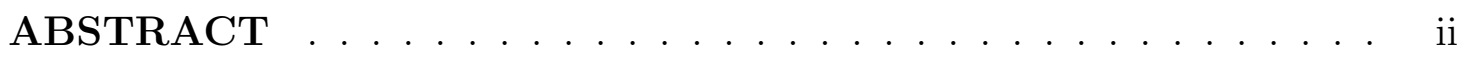

ACKNOWLEDGMENTS ................ iii

TABLE OF CONTENTS $\ldots \ldots \ldots \ldots \ldots \ldots$ iv

LIST OF FIGURES . . . . . . . . . . . . . . vii

LIST OF TABLES ................... . ix

\section{CHAPTER}

1 Introduction . . . . . . . . . . . . . . . 1

1.1 Overview of Thesis $\ldots \ldots \ldots \ldots \ldots \ldots$

2 Review of Literature . . . . . . . . . . . . . 4

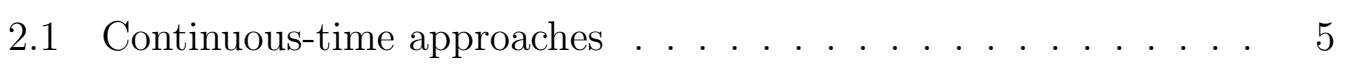

2.2 Discrete-time approaches . . . . . . . . . . . . . . . 11

3 Stable MIMO System Inversion $\ldots \ldots \ldots \ldots$

3.1 Feedback Tracking System _ . . . . . . . . . . . . . . 16

3.2 Algorithm for Stable System Inversion _ . . . . . . . . . 21

3.2.1 Eigenvalues of the Inverse Filter . . . . . . . . . . . 23

3.2 .2 Adding Additional Advances . . . . . . . . . . . . 27

3.2.3 Extension to Feedback Approach . . . . . . . . . 30

3.3 Command Shaping Filter . . . . . . . . . . . . . . . 34

3.4 Inverse Modified Plant . . . . . . . . . . . . . . 37

3.5 Inclusion of a State Observer . . . . . . . . . . . . . . . 39 


\section{Page}

4 Feedback Inversion Design Techniques . . . . . . . . . . . . 42

4.1 Classical Feedback Design . . . . . . . . . . . . . . 43

4.2 Norm Minimization Approach . . . . . . . . . . . . . . . 44

4.2.1 Constraints for the Feedback Gain Matrix . . . . . . . . 45

4.2.2 Design by Optimization . . . . . . . . . . . . 50

4.2.3 Discussion of the Approach . . . . . . . . . . . . 55

4.3 Frequency Optimization Approach . . . . . . . . . . . . 56

4.3.1 Main Diagonal Elements . . . . . . . . . . . . 57

4.3.2 Off-Diagonal Elements . . . . . . . . . . . 58

4.3.3 Stability Constraint . . . . . . . . . . . 59

4.3.4 Discussion of the Approach . . . . . . . . . . 60

5 Example Systems and Simulation Results . . . . . . . . . 62

$5.1 \quad$ H-Frame System . . . . . . . . . . . . . . . . . . . . . 68

5.2 Atomic Force Microscope . . . . . . . . . . . . . . . . . 79

5.3 Scanning Tunneling Microscope . . . . . . . . . . . . . . . 93

5.4 Bell 205 Helicopter . . . . . . . . . . . . . . . . . . 96

5.5 Multilink Flexible Manipulator . . . . . . . . . . . . . . . . . . . 102

5.6 Overhead Crane . . . . . . . . . . . . . . . . 107

5.7 Two Discs . . . . . . . . . . . . . . . . . . . . . 112

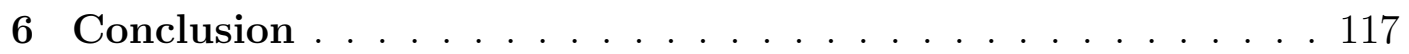

6.1 Areas of Future Work . . . . . . . . . . . . . . . . 118

LIST OF REFERENCES . . . . . . . . . . . . . . . . . . 120

\section{APPENDIX}


Page

Derivation of the Dual Problem . . . . . . . . . . . . . . . 124

BIBLIOGRAPHY . . . . . . . . . . . . . . . 133 


\section{LIST OF FIGURES}

$\begin{array}{lll}\text { Figure } & \text { Page }\end{array}$

2.1 Tracking architectures ................ 5

$2.2 \quad$ Partitioned SISO system . . . . . . . . . . . . . . 11

2.3 Inverse Dynamic Control Architecture (according to [1]) . . . . 13

$3.1 \quad$ A digital state-space tracking system . . . . . . . . . . . . 17

3.2 Input-multiplicative stability robustness bound . . . . . . . . 20

3.3 Input-feedback plant perturbation model . . . . . . . . . . . . . 21

$3.4 \quad$ Pole radius for a stable $\Phi_{c} \ldots \ldots$. . . . . . . . . . . 30

3.5 Precision Tracking System - Command Shaping Filter .... 37

$3.6 \quad$ Precision Tracking System - Inverse Modified Plant . . . . . . . 38

5.1 Frequency response of the H-Frame precision tracking system comparing a standard feedback tracking system (FTS) without feedforward, and the same feedback system with a feedforward CSF filter . . . . . . . . . . . . . . . 70

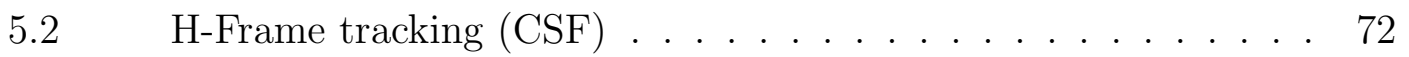

5.3 Frequency response of the H-Frame precision tracking system (CSF - frequency optimization) . . . . . . . . . . 73

$5.4 \quad$ H-Frame tracking (CSF - frequency optimization) . . . . . . . . 74

5.5 H-Frame tracking (CSF - with observer) . . . . . . . . 76

5.6 H-Frame tracking (CSF - observer with uncertainties) . . . . 78

5.7 Frequency response of the H-Frame precision tracking system (IMP) . . . . . . . . . . . . . . . . . . . 80

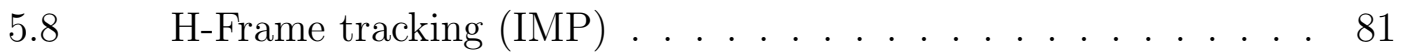

5.9 Frequency response of the AFM precision tracking system (CSF) 84 
5.10 AFM tracking $(\mathrm{CSF}) \ldots \ldots \ldots . \ldots . \ldots . \ldots 4$

5.11 Frequency response of the AFM precision tracking system (CSF - frequency optimization) . . . . . . . . . . 85

5.12 AFM tracking (CSF - frequency optimization) . . . . . . 86

5.13 AFM tracking (CSF - uncertain plant with observer) . . . . 88

5.14 Frequency response of the AFM precision tracking system (IMP) 90

5.15 AFM tracking (IMP) ................... 91

$5.16 \quad$ STM reference trajectories . . . . . . . . . . . 95

5.17 Helicopter reference trajectory for $y_{2}$ and $y_{3} \ldots \ldots$. . . . . 98

5.18 Pole zero map of the continuous-time flexible manipulator plant 103

5.19 Overhead crane reference trajectory . . . . . . . . . . 110

5.20 Two discs reference trajectory . . . . . . . . . . . . 114 


\section{LIST OF TABLES}

Table

Page

5.1 Normalized Bessel poles for 1st through 6th-order systems with 1-second settling time. To get a settling time of $T_{S}$ seconds, divide all poles by $T_{S}$. . . . . . . . . . . . . . . . 62

$5.2 \quad$ H-Frame tracking results . . . . . . . . . . . . . . . . . 79

5.3 AFM tracking results . . . . . . . . . . . . . . . 92

5.4 STM tracking results . . . . . . . . . . . . 96

5.5 Bell 205 Helicopter tracking results . . . . . . . . . . . . 101

5.6 Multilink Flexible Manipulator tracking results . . . . . . . . 106

5.7 Overhead Crane parameters ............... 108

5.8 Overhead Crane tracking results . . . . . . . . . . . . 111

5.9 Two Discs tracking results . . . . . . . . . . . . 116 


\section{CHAPTER 1}

\section{Introduction}

Tracking is often used to achieve zero steady-state error for control systems. This thesis, however, focuses on the transient response of control systems, and does not only care about the steady-state error. For this reason, the approach is called precision tracking because it is designed to precisely follow the reference trajectory

during all time. In order to reach this goal, some kind of system inversion is required.

If the system under consideration is non-minimum phase, the realization of an exact inversion of the system is not used since this scenario leads to unstable eigenvalues in the inverted system. Therefore, approximations are needed. In this thesis, two architectures for the approximate inversion of discrete-time linear systems will be considered. Even if the continuous-time system is minimum phase, the corresponding discrete-time system can be non-minimum phase, due to the presence of sampling zeros (for example there will always be zeros outside the unit circle if the relative degree of the continuous-time system is greater than or equal to three, and the sampling period is sufficiently short [2]).

Classic feedback control systems with integral control may face the problem of having only a small bandwidth for precision tracking, since they only guarantee zero steady-state error. This work will address this problem by using and designing a feedforward filter to improve the precision tracking bandwidth of the control system.

Precision tracking is important in many applications such as atomic force microscopes (AFM) [3, 4, 5], scanning tunneling microscopes (STM) [6], piezoelectricstack actuated nanopositioning platforms [7, 8] and hypersonic vehicles [9]. Two 
of the applications will be briefly explained below to demonstrate the importance of precision tracking.

The AFM is a versatile instrument which is able to image nanoscale structures with an increasing importance in molecular biology [10]. It is particularly interesting for control engineers since the imaging depends utterly on the feedback control loop. Advantages of the AFM over optical methods comprise a higher resolution and the fact that the AFM produces a 3D surface map. The applications include material sciences (photovoltaic cells, crystallization, semiconductor properties) and the scan of biologically relevant materials (membrane stability, cell motility) [10]. The AFM can also be used to manipulate material, making it a useful actuator for nanotechnology. The quality and speed of AFM images is dependent on the overall dynamics of the AFM system [4], hence a good tracking of the reference trajectory is desired.

The STM is an important tool in nanofabrication, but its problem is that it cannot compete with more established techniques due to its limited operating speed (throughput) [6]. The throughput, however, is limited because of positioning errors in the STM system, and therefore a high-speed precision positioning system is required.

\section{$1.1 \quad$ Overview of Thesis}

The thesis is structured as follows: Chapter 2 gives an overview of the existing literature.

Next, in Chapter 3, the considered approaches to invert a given system are explained: two different ways to derive a stable feedforward filter which approximately inverts the system are presented. Starting point is the exact, unstable feedforward filter. The first design possibility relies on considering a certain number of advances for the system outputs to come up with a stable filter, while the 
other technique focuses on designing feedback to stabilize the filter. In the context of these inversion techniques, two different precision tracking architectures will be presented.

In Chapter 4, several alternatives to calculate the mentioned feedback controller are presented. Among these alternatives, especially an idea to formulate an optimization problem to obtain an ideal feedback gain matrix which optimizes the system's frequency response excels.

Chapter 5 introduces various example systems which are to be inverted for precision tracking and provides simulation results. Finally, Chapter 6 gives the conclusion. 


\section{CHAPTER 2}

\section{Review of Literature}

Inversion of non-minimum phase systems has been studied previously by various authors. First, some basic thoughts on the inversion of non-minimum phase systems are presented. In the following sections, a few approaches and ideas shall be discussed, together with a (personal) evaluation of the advantages and disadvantages between these approaches and the method derived in this thesis.

Different kinds of inversion approaches and architectures can be distinguished and exist in the literature. In general, inversion approaches can be split up into closed-loop inversion feedforward (CLIF) and plant-inversion feedforward (PIF) architecures, as shown in Fig $2.1[11,12]$. The inverse filter is denoted by $\mathbf{F}, \mathbf{G}_{c}$ is a feedback controller and $\mathbf{G}_{p}$ a plant model. The goal of the architectures is to track the desired output $\boldsymbol{y}_{d}$ with the actual plant output $\boldsymbol{y}$.

Moreover, different approaches to find the inverse filter can be distinguished. On the one hand, various stable approximate model-inversion techniques exist [11, 13] (e.g. Tomizuka's zero phase error tracking control [14]), especially for discrete-time, single-input, single-output (SISO) plants. These approaches try to exactly invert the system model, but replace the unstable part of the zero dynamics with a stable approximation [13]. On the other hand, techniques have been published which use the exact, unstable inversion and ensure stability of the system by using noncausal plant inputs [13] (e.g. in $[15,6,16])$. Some of the noncausal approaches use some kind of preview time to make the inversion more applicable to applications where the desired output trajectory is not completely pre-specified [15].

An aspect that many inversion approaches (both continuous- and discrete- 


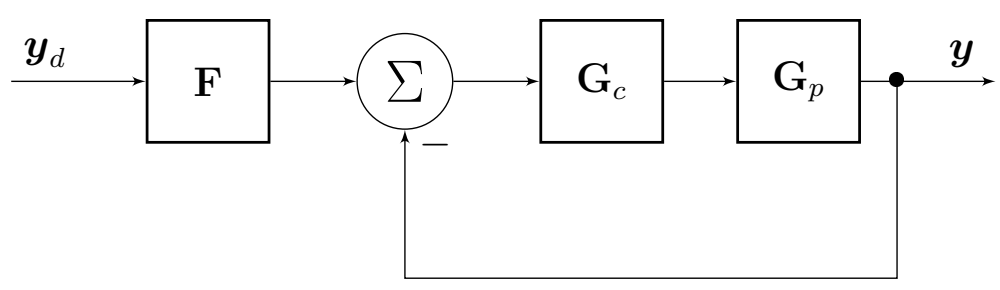

(a) Closed-loop inversion feedforward (CLIF) architecture

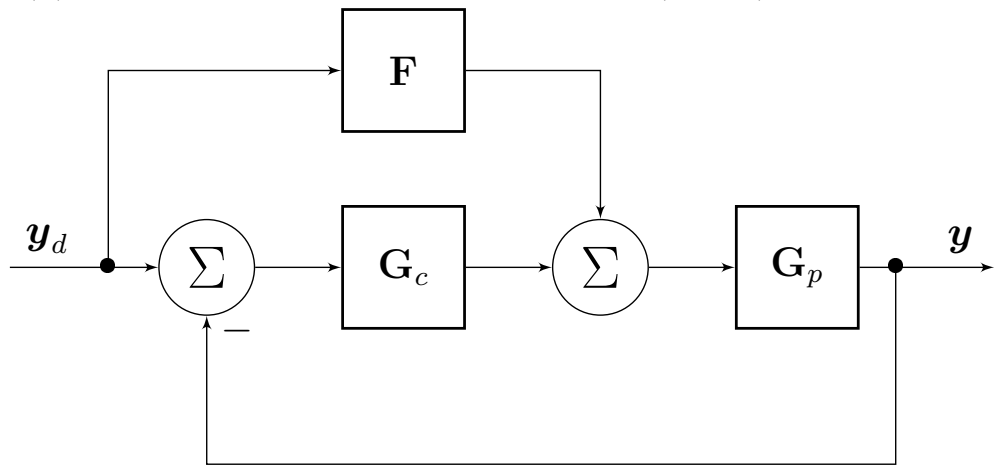

(b) Plant inversion feedforward (PIF) architecture

Figure 2.1: Tracking architectures

time) have in common is that they specifically exclude systems with a transmission zero on the imaginary axis or on the unit circle, respectively (e.g. [15, 6, 17, 16, 18]).

The inverse filter that will be derived in Chapter 3 is an approximate inverse for a discret-time non-minimum phase system which uses an advanced version of the reference input (i.e. advanced by a finite number of samples) to drive the inverse filter.

\subsection{Continuous-time approaches}

One continuous-time approach can be found in [15]. It calculates the feedforward input that leads to precision output tracking. The method will be discussed in more detail here.

The starting point is the square state-space system

$$
\begin{aligned}
& \dot{\boldsymbol{x}}(t)=\mathbf{A} \boldsymbol{x}(t)+\mathbf{B} \boldsymbol{u}(t) \\
& \boldsymbol{y}(t)=\mathbf{C} \boldsymbol{x}(t)
\end{aligned}
$$


with $\boldsymbol{x}(t) \in \mathbb{R}^{n}$ and $\boldsymbol{u}(t), \boldsymbol{y}(t) \in \mathbb{R}^{p}$. First, the relation

$$
\boldsymbol{y}^{(\boldsymbol{r})}(t)=\mathbf{A}_{x} \boldsymbol{x}(t)+\mathbf{B}_{y} \boldsymbol{u}(t)
$$

with

$$
\mathbf{A}_{x}=\left[\begin{array}{c}
\boldsymbol{c}_{1}^{\mathrm{T}} \mathbf{A}^{r_{1}} \\
\vdots \\
\boldsymbol{c}_{p}^{\mathrm{T}} \mathbf{A}^{r_{p}}
\end{array}\right] \quad \mathbf{B}_{y}=\left[\begin{array}{c}
\boldsymbol{c}_{1}^{\mathrm{T}} \mathbf{A}^{r_{1}-1} \mathbf{B} \\
\vdots \\
\boldsymbol{c}_{p}^{\mathrm{T}} \mathbf{A}^{r_{p}-1} \mathbf{B}
\end{array}\right]
$$

and

$$
\boldsymbol{r}=\left[\begin{array}{lll}
r_{1} & \ldots & r_{p}
\end{array}\right]^{\mathrm{T}}
$$

has to be found. This relation motivates

$$
\boldsymbol{u}_{f f}(t)=\mathbf{B}_{y}^{-1}\left(\boldsymbol{y}_{d}^{(\boldsymbol{r})}(t)-\mathbf{A}_{x} \boldsymbol{x}(t)\right)
$$

as the choice of the control law $\boldsymbol{u}(t)$, where the term $\boldsymbol{y}_{d}^{(\boldsymbol{r})}$ is defined by $\boldsymbol{y}_{d}^{(\boldsymbol{r})}=$ $\left[\begin{array}{lll}\frac{d^{r} y_{d, 1}}{d t^{r} 1} & \ldots & \frac{d^{r} y_{d, p}}{d t^{r p}}\end{array}\right]^{\mathrm{T}}$ and $\boldsymbol{y}_{d}(t)$ is the desired output trajectory.

The next step is to introduce a transformation $\mathbf{T}$ to divide the state vector $\boldsymbol{x}(t)$ into a known part $\boldsymbol{\xi}(t)$, which depends on the desired output trajectory $\boldsymbol{y}_{d}(t)$, and the internal dynamics $\boldsymbol{\eta}(t)$, i.e. $\left[\begin{array}{ll}\boldsymbol{\xi}(t) & \boldsymbol{\eta}(t)\end{array}\right]^{\mathrm{T}}=\mathbf{T} \boldsymbol{x}(t)$. The part $\boldsymbol{\xi}(t)$ is given by

$$
\boldsymbol{\xi}(t)=\left[\begin{array}{lllllllll}
y_{1} & \dot{y_{1}} & \ldots & \frac{d^{r_{1}-1} y_{1}}{d t^{r_{1}-1}} & \ldots & y_{p} & \dot{y_{p}} & \ldots & \frac{d^{r_{p}-1} y_{p}}{d t^{r_{p}-1}}
\end{array}\right]^{\mathrm{T}}
$$

so the control law becomes

$$
\boldsymbol{u}_{f f}(t)=\mathbf{B}_{y}^{-1}\left(\boldsymbol{y}_{d}^{(\boldsymbol{r})}(t)-\mathbf{A}_{\xi} \boldsymbol{\xi}_{d}(t)-\mathbf{A}_{\eta} \boldsymbol{\eta}(t)\right)
$$

where $\boldsymbol{\xi}_{d}(t)$ is the desired $\boldsymbol{\xi}(t)$, which is known when the desired output and its time derivatives are specified.

Finally, the internal dynamics $\boldsymbol{\eta}(t)$ have to be calculated. If the system is hyperbolic (i.e. none of the zeros of the system is located on the imaginary axis), the internal dynamics can be decoupled with a transformation matrix $\mathbf{U}$ into a 
stable $\left(\boldsymbol{\sigma}_{s}(t)\right)$ and an unstable $\left(\boldsymbol{\sigma}_{u}(t)\right)$ subsystem

$$
\begin{aligned}
\dot{\boldsymbol{\sigma}}_{s}(t) & =\overline{\mathbf{A}}_{s} \boldsymbol{\sigma}_{s}(t)+\overline{\mathbf{B}}_{s} \boldsymbol{Y}_{d}(t) \\
\dot{\boldsymbol{\sigma}}_{u}(t) & =\overline{\mathbf{A}}_{u} \boldsymbol{\sigma}_{u}(t)+\overline{\mathbf{B}}_{u} \boldsymbol{Y}_{d}(t)
\end{aligned}
$$

where $\boldsymbol{Y}_{d}(t)=\left[\begin{array}{ll}\boldsymbol{y}_{d}^{(\boldsymbol{r})}(t) & \boldsymbol{\xi}_{d}(t)\end{array}\right]^{\mathrm{T}}$. In this hyperbolic case, the solutions for the internal dynamics are given by

$$
\begin{aligned}
& \boldsymbol{\sigma}_{s}(t)=\int_{-\infty}^{t} \mathrm{e}^{\overline{\mathbf{A}}_{s}(t-\tau)} \overline{\mathbf{B}}_{s} \boldsymbol{Y}_{d}(\tau) \mathrm{d} \tau \\
& \boldsymbol{\sigma}_{u}(t)=-\int_{t}^{\infty} \mathrm{e}^{-\overline{\mathbf{A}}_{u}(\tau-t)} \overline{\mathbf{B}}_{u} \boldsymbol{Y}_{d}(\tau) \mathrm{d} \tau
\end{aligned}
$$

i.e. the internal dynamics $\boldsymbol{\eta}(t)$ can be calculated (if the system is non-hyperbolic, the author developed an idea to deal with this case in [19]). It is important to notice that the desired output must be completely specified (including future information) in order to compute the solution for the unstable part $\boldsymbol{\sigma}_{u}(t)$. For online computation, it is assumed that $\boldsymbol{y}_{d}$ is known for a preview time of $T_{p}$ seconds, i.e. $\boldsymbol{Y}_{d}(\tau)$ is known for all $t \leq \tau \leq t+T_{p}$, and

$$
\overline{\boldsymbol{\sigma}}_{u}(t)=-\int_{t}^{t+T_{p}} \mathrm{e}^{-\overline{\mathbf{A}}_{u}(\tau-t)} \overline{\mathbf{B}}_{u} \boldsymbol{Y}_{d}(\tau) \mathrm{d} \tau
$$

is the approximate solution for the internal dynamics. It can be shown that it is possible to make $\boldsymbol{\sigma}_{u}(t)-\overline{\boldsymbol{\sigma}}_{u}(t)$ arbitrarily small by having a large enough preview time $T_{p}$. The proposed method was tested on an experimental flexible structure consisting of two discs, which are connected by a thin freely rotating shaft. The input of the system is the voltage applied to a DC motor, and the output is the angular rotation of the second disc. The method was able to track the desired output signal, and increasing the preview time improved the tracking performance. It was also possible to specify the trajectory online.

A similar approach by the same authors is presented in [6]. The starting point is the same as above, i.e. a square continuous-time state-space system, with the 
Laplace-domain representation given by

$$
\boldsymbol{y}(s)=\mathbf{C}(s \mathbf{I}-\mathbf{A})^{-1} \mathbf{B} \boldsymbol{u}(s)=\mathbf{G}(s) \boldsymbol{u}(s) .
$$

The optimal inversion problem is formulated as an optimization problem. The goal is to minimize the cost function

$$
J=\int_{-\infty}^{\infty}\left\{\boldsymbol{u}^{*}(j \omega) \mathbf{R}(j \omega) \boldsymbol{u}(j \omega)+\left[\boldsymbol{y}(j \omega)-\boldsymbol{y}_{d}(j \omega)\right]^{*} \mathbf{Q}(j \omega)\left[\boldsymbol{y}(j \omega)-\boldsymbol{y}_{d}(j \omega)\right]\right\} \mathrm{d} \omega
$$

where $\mathbf{R}(j \omega)$ is a weight on the input energy and $\mathbf{Q}(j \omega)$ a weight on the outputtracking error (both frequency-dependent). The corresponding optimal inverse is given as the filter $\mathbf{G}_{\mathrm{opt}}(s)$

$$
\mathbf{G}_{\mathrm{opt}}(s)=\left[\mathbf{R}(s)+\mathbf{G}^{\mathrm{T}}(-s) \mathbf{Q}(s) \mathbf{G}(s)\right]^{-1} \mathbf{G}^{\mathrm{T}}(-s) \mathbf{Q}(s)
$$

with

$$
\boldsymbol{u}_{\mathrm{opt}}(j \omega)=\mathbf{G}_{\mathrm{opt}}(j \omega) \boldsymbol{y}_{d}(j \omega)
$$

The problem with this filter is that it tends to be unstable if the system under consideration is non-minimum phase. Due to the non-causality of the filter, a preview-based implementation approach for the optimal inverse filter is developed. First, the filter has to be rewritten

$$
\boldsymbol{u}_{\mathrm{opt}}(s)=\mathbf{G}_{\mathrm{opt}}(s) \boldsymbol{y}_{d}(s)=\hat{\mathbf{G}}_{\mathrm{opt}}(s) \hat{\boldsymbol{y}}_{d}(s)
$$

such that $\hat{\mathbf{G}}_{\mathrm{opt}}(s)$ is proper and $\hat{\boldsymbol{y}}_{d}(s)$ is the Laplace transformation of a linear combination of the desired output and its time derivatives. Next, the filter is decoupled into a stable $\left(\mathbf{G}_{\mathrm{opt}}^{s}(s)\right)$ and an unstable $\left(\mathbf{G}_{\mathrm{opt}}^{u}(s)\right)$ part

$$
\hat{\mathbf{G}}_{\mathrm{opt}}(s)=\mathbf{G}_{\mathrm{opt}}^{s}(s)+\mathbf{G}_{\mathrm{opt}}^{u}(s)
$$

by partial fraction expansion. Let the state-space representations be given by

$$
\begin{aligned}
\dot{\boldsymbol{x}}_{s}(t) & =\mathbf{A}_{s} \boldsymbol{x}_{s}(t)+\mathbf{B}_{s} \hat{\boldsymbol{y}}_{d}(t) \\
\boldsymbol{u}_{\mathrm{opt}}^{s}(t) & =\mathbf{C}_{s} \boldsymbol{x}_{s}(t)+\mathbf{D}_{s} \hat{\boldsymbol{y}}_{d}(t)
\end{aligned}
$$


and

$$
\begin{gathered}
\dot{\boldsymbol{x}}_{u}(t)=\mathbf{A}_{u} \boldsymbol{x}_{u}(t)+\mathbf{B}_{u} \hat{\boldsymbol{y}}_{d}(t) \\
\boldsymbol{u}_{\mathrm{opt}}^{u}(t)=\mathbf{C}_{u} \boldsymbol{x}_{u}(t)+\mathbf{D}_{u} \hat{\boldsymbol{y}}_{d}(t) .
\end{gathered}
$$

If the desired output and its time derivatives $\hat{\boldsymbol{y}}_{d}(t)$ are bounded in time, the bounded solution to the optimal-inverse input $\boldsymbol{u}_{\mathrm{opt}}(t)$ is found as

$$
\begin{aligned}
& \boldsymbol{u}_{\mathrm{opt}}^{s}(t)=\mathbf{C}_{s} \int_{-\infty}^{t} \mathrm{e}^{\mathbf{A}_{s}(t-\tau)} \mathbf{B}_{s} \hat{\boldsymbol{y}}_{d}(\tau) \mathrm{d} \tau+\mathbf{D}_{s} \hat{\boldsymbol{y}}_{d}(\tau) \\
& \boldsymbol{u}_{\mathrm{opt}}^{u}(t)=-\mathbf{C}_{u} \int_{t}^{\infty} \mathrm{e}^{\mathbf{A}_{u}(\tau-t)} \mathbf{B}_{u} \hat{\boldsymbol{y}}_{d}(\tau) \mathrm{d} \tau+\mathbf{D}_{u} \hat{\boldsymbol{y}}_{d}(\tau) \\
& \boldsymbol{u}_{\mathrm{opt}}(t)=\boldsymbol{u}_{\mathrm{opt}}^{s}(t)+\boldsymbol{u}_{\mathrm{opt}}^{u}(t) .
\end{aligned}
$$

The computation of the optimal-inverse input, at any time $t$, requires the knowledge of all future values of the desired output trajectory $\boldsymbol{y}_{d}(t)$. The input can be approximated by using a finite preview time $T_{p}$, so that the values are known within the time interval $\left[t, t+T_{p}\right]$. The approximation for the unstable part becomes

$$
\tilde{\boldsymbol{u}}_{\mathrm{opt}}^{u}(t)=-\mathbf{C}_{u} \int_{t}^{t+T_{p}} \mathrm{e}^{\mathbf{A}_{u}(\tau-t)} \mathbf{B}_{u} \hat{\boldsymbol{y}}_{d}(\tau) \mathrm{d} \tau+\mathbf{D}_{u} \hat{\boldsymbol{y}}_{d}(\tau)
$$

and

$$
\tilde{\boldsymbol{u}}_{\mathrm{opt}}(t)=\boldsymbol{u}_{\mathrm{opt}}^{s}(t)+\tilde{\boldsymbol{u}}_{\mathrm{opt}}^{u}(t)
$$

is the result for the finite preview based optimal-inverse input. It is shown that the tracking error can be made arbitrarily small by choosing a sufficiently large preview time. A rule of thumb is introduced as well, the preview time $T_{p}$ should be "greater than four times the time constant of of the dominant unstable pole" of the optimal inversion filter $\mathbf{G}_{\text {opt }}(s)$. This method was applied to the STM system, and compared to a "dc-gain approach", where the desired trajectory is scaled by the dc gain of the STM model. The optimal-inverse can greatly improve the tracking performance of the STM system in comparison to the dc-gain approach, when using a large enough preview time. An insufficient preview time, however, can lead to substantial tracking errors. An advantage of the method considered in this thesis 
in comparison to this continuous-time approach may be that neither in [15] nor in [6] the author says anything about the digital implementation of the approach, e.g. the sampling rate necessary to perform the integral calculations with sufficient accuracy.

Another continuous-time approach can be found in [9]. It deals with the control of an unstable, non-minimum phase hypersonic vehicle model. The basic idea of the approach is to add a stabilizing term to a standard dynamic inversion method to move the unstable zero into the left-half plane. The proposed method is valid for multiple-input, multiple-output (MIMO) systems. One disadvantage of the approach is that it involves a transformation to Jordan form, which can be difficult in practice, especially when the matrix which is to be converted possesses multiple eigenvalues.

In [20], an approximate-inverse method for SISO systems (that can also include a term $\left.G_{0}(s)=e^{-s T_{0}}\right)$ is presented, which uses no preview time and a causal inverse control law. For this method, the desired output trajectory must be known at any time instant $t \geq 0$. The perfect inversion control law for a plant $G(s)$ with $m$ transmission zeros is found by partitioning the plant:

$$
G(s)=G_{10}(s)\left(b_{m} s^{m}+\cdots+b_{1} s+b_{0}\right) .
$$

The structure can be seen in Fig. 2.2, and the ideal feedforward inversion control law follows as

$$
u_{\mathrm{FF}}=c_{n_{\nu}} y_{1 d}^{\left(n_{\nu}\right)}+\cdots+c_{1} y_{1 d}^{(1)}+c_{0} y_{1 d}
$$

where the coefficients $c_{k}, k=1, \ldots, n_{\nu}$ are determined by the coefficients in $G_{10}(s)$ $\left(n_{\nu}\right.$ depends on the system order and the order of the Taylor approximation of $\left.G_{0}(s)=e^{-s T_{0}}\right)[20]$. The problem is that (2.23) cannot be used, since signals $y_{1 d}^{(k)}$ are not available for a designer. The paper focuses on finding good approximations for this control law. An advantage of this approach is that it specifically includes 


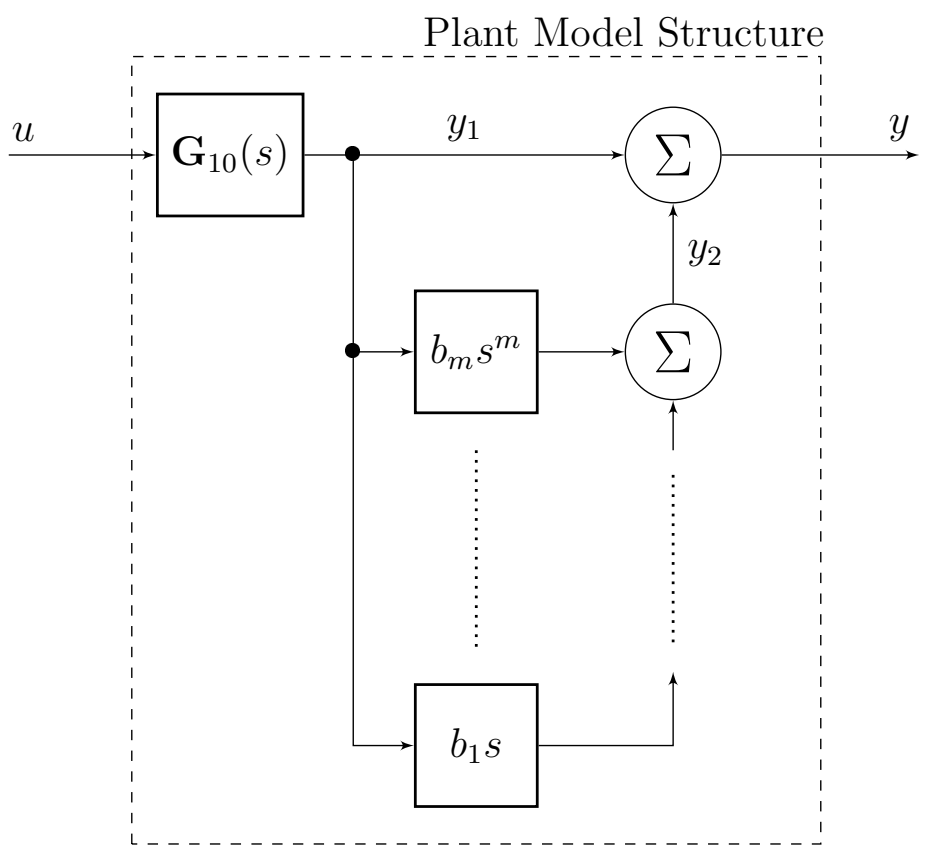

Figure 2.2: Partitioned SISO system

a term $G_{0}(s)=e^{-s T_{0}}$, which is not the case for the method in Chapter 3, but the author does not generalize the approach to MIMO systems.

\subsection{Discrete-time approaches}

The theoretical problem of inverting non-minimum phase discrete-time MIMO systems is solved in [16]. It is shown, however, that the perfect inversion requires an infinite number of "preaction" samples. Almost perfect tracking can be achieved if a preview of the reference trajectory is available that is "significantly greater than the maximum time constant associated to the inverses of the controlled system invariant zeros".

One of the standard approaches for discrete-time non-minimum phase SISO systems, the zero phase error tracking control (ZPETC), was presented in [14]. The method designs a feedforward controller for a closed loop system, where a combination of pole/zero cancellation and phase cancellation (applied to uncancellable zeros) is used in order to ensure that the desired and the actual output are 
in phase for all frequencies. In [1], a filter is proposed to ensure unity gain, instead of zero phase. The ZPETC could be successfully applied to motion control of a robot arm, excelling both in terms of tracking error and smoothness of velocity. Unfortunately, the original ZPETC, as proposed in [14], is sensitive to modeling errors and plant uncertainties, whereas the method from Chapter 3 explicitly deals with stability robustness. Therefore, an adaptive ZPETC was presented in [21].

In [17], a trajectory tracking control for non-minimum phase SISO systems is introduced by factorizing the system into a minimum phase and a zero phase system. By inverting both systems individually, a feedforward controller can be constructed. For the zero phase system, a discrete zero-phase FIR filter was designed. The method was tested successfully on the mock model of a XY gantry stage.

A different approach for perfect tracking for discrete-time systems was proposed in [22]. The authors eliminate the unstable zero problem in the design of the inverse discrete-time system by using a multirate feedforward controller. A highly robust performance is achieved by the controller. Advantages of the method in comparison to ZPETC could be shown through simulation and experiments, in the context of a position control using a de servomotor.

An approach for a filter design using optimization can be found in [1]. It is called model matching and the basic idea is to find a filter $F$ which minimizes the worst case frequency domain gain deviation, i.e.

$$
F=\arg \min _{F \in \mathcal{R} \mathcal{H} \infty}\|G F-W\|_{\infty}
$$

where $G$ is the non-minimum phase plant and $W$ a weight chosen based on the frequencies contained in the desired output. A choice of $W=1$ for all frequencies would cause difficulties, since the minimization will try to approach $F=G^{-1}$. Instead, $W$ should be chosen close to unity for the frequency range of interest. 


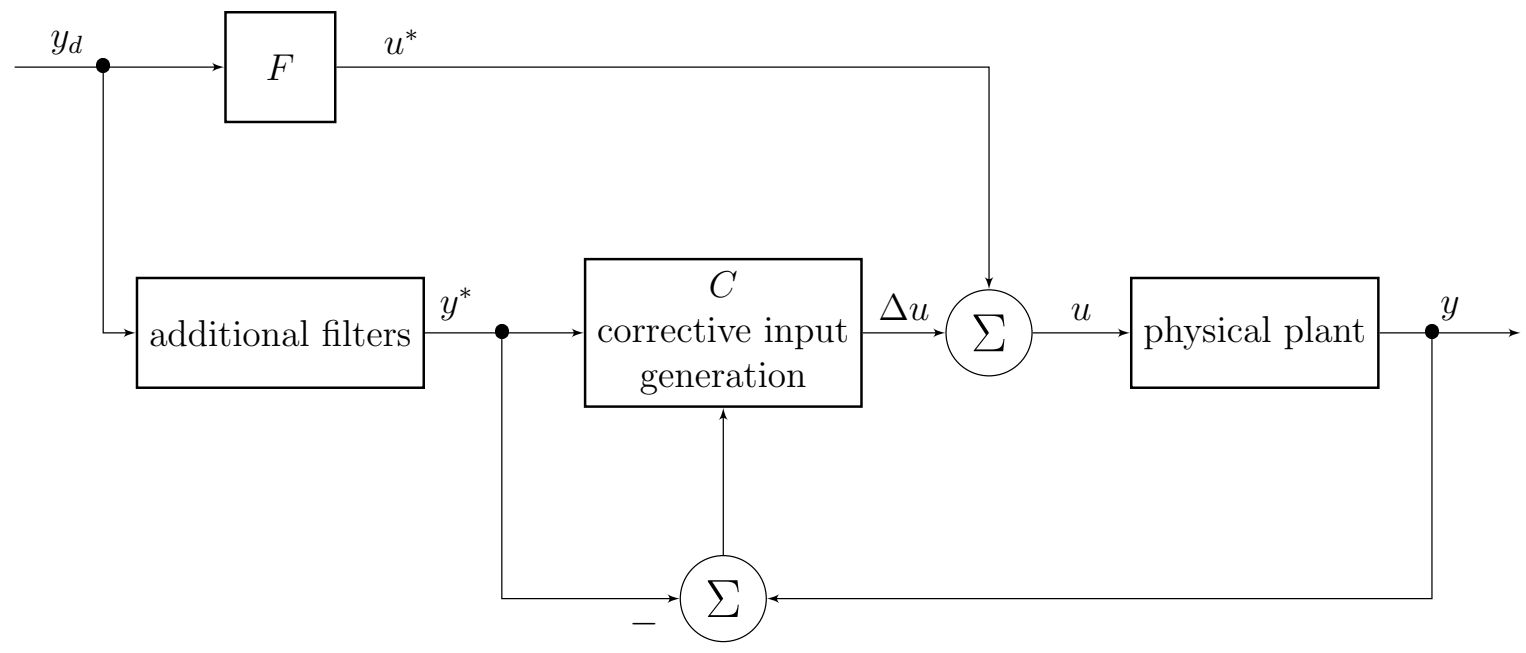

Figure 2.3: Inverse Dynamic Control Architecture (according to [1])

An idea from the same authors to improve robustness can be found in $[1$, 23]. The first step is to design an inverse dynamic filter $F$ for a feedback control system (the papers propose several approaches for that filter, e.g. ZPETC or model matching). The authors' concern, however, derives from an unsatisfactory filter performance due to a mismatch between the model and the physical system. Therefore, they propose an iterative refinement approach to update the input of the control system based on the output tracking error. An initial input sequence $\boldsymbol{u}^{*}$

$$
\boldsymbol{u}^{*}:=\left\{u^{*}\left(t_{i}\right): i \in 0,1, \ldots, N\right\}
$$

is given, which is called the nominal command input. It derives from the filter $F$.

A gradient descent approach was used to develop an algorithm for the update process. The algorithm takes the input of the proceeding loop and updates it in the following way:

$$
u_{\text {new }}=u_{\text {old }}+\Delta u \text {. }
$$

Assume a SISO system with its transfer function $G$ (from input $u$ to output $y$ ), where the feedback-loop is already included. Given is a desired output sequence 
$y^{*}$

$$
\boldsymbol{y}^{*}:=\left\{y^{*}\left(t_{i}\right): i \in 0,1, \ldots, N\right\}
$$

The starting point of the algorithm is to set $\boldsymbol{u}=\boldsymbol{u}^{*}$, and it works as follows:

1. Apply $\boldsymbol{u}$ to physical system and obtain output sequence $\boldsymbol{y}$ :

$$
\boldsymbol{y}:=\left\{y\left(t_{i}\right): i \in 0,1, \ldots, N\right\}
$$

2. Update $\boldsymbol{u}$ by adding a corrective term $\Delta \boldsymbol{u}$ :

$$
\Delta \boldsymbol{u}=-\underbrace{\alpha}_{\text {can be found by a line search adjoint of } G} \underbrace{G^{*}}\left(\boldsymbol{y}-\boldsymbol{y}^{*}\right)
$$

3. Iterate until $\left\|\boldsymbol{y}-\boldsymbol{y}^{*}\right\|$ or $\|\Delta \boldsymbol{u}\|$ becomes sufficiently small

The architecture can be seen in Fig. 2.3. For the update process, not the ideal desired output trajectory $y_{d}$ is used, since the use of it turned out to be too aggressive and leading to saturation. Instead, filtering is applied to $y_{d}$ (e.g $y^{*}=$ $G u^{*}$ could be used).

For this algorithm, a complete run of the control system is needed in order to make one update. For a real-time implementation, it is proposed by the authors to use the results from the iterative refinement to train a FIR filter mapping $y_{d}$ to the corrective input $\Delta \boldsymbol{u}$ :

$$
\Delta u_{k}=w_{1} y_{k+n_{1}}^{*}+w_{2} y_{k+n_{1}-1}^{*}+\cdots+w_{n} y_{k-n_{2}+1}^{*}
$$

with $n=n_{1}+n_{2}$ as the order of the filter and $n_{1}$ as the look-ahead horizon. The filter coefficients can be obtained through a least square fit to the data obtained from the iterative refinement. The method was tested on a high speed positioning system. The system model was obtained by identification. The fixed-coefficient FIR filter was able to improve the tracking performance. The tracking error was 
significantly and uniformly reduced in comparison to the case where just the inverse dynamic filter was used. An advantage of the proposed inversion method in Chapter 3 could be that, as mentioned earlier, the stability robustness is taken into account while designing the entire inversion-based tracking system, without running an algorithm to update the ideal inversion input. 


\section{CHAPTER 3}

\section{Stable MIMO System Inversion}

In the following chapter, the tracking architectures that were examined and derived in the course of this thesis will be presented. They were first introduced in $[24,25]$. The design of digital control systems for precision tracking will be discussed. The first step is to design a feedback control system using classic poleplacement or linear-quadratic regulator techniques, together with integral control, so a stable closed-loop system having zero steady-state error to step inputs is guaranteed. In order to have precision tracking for other types of inputs, different kinds of feedforward filters for the closed-loop system will be derived, based on a novel approximation. If the closed-loop system is non-minimum phase, the filter and the closed-loop system are approximately a decoupled system of delays over a certain bandwidth. Moreover, an alternative feedback design technique to stabilize the inverse filter is considered and presented.

\subsection{Feedback Tracking System}

The first step is to design a 2-degree-of-freedom feedback control system in order to achieve a desired bandwidth with good stability robustness.

Consider an $n$ th-order linear, $m$-input, $p$-output plant with state-space model

$$
\begin{aligned}
& \dot{\boldsymbol{x}}(t)=\mathbf{A} \boldsymbol{x}(t)+\mathbf{B} \boldsymbol{u}(t) \\
& \boldsymbol{y}(t)=\mathbf{C} \boldsymbol{x}(t) .
\end{aligned}
$$

For digital control with sampling time $T$ seconds the zero-order hold $(\mathrm{ZOH})$ equivalent plant model is

$$
\begin{aligned}
\boldsymbol{x}[k+1] & =\boldsymbol{\Phi} \boldsymbol{x}[k]+\boldsymbol{\Gamma} \boldsymbol{u}[k] \\
\boldsymbol{y}[k] & =\mathbf{C} \boldsymbol{x}[k]
\end{aligned}
$$




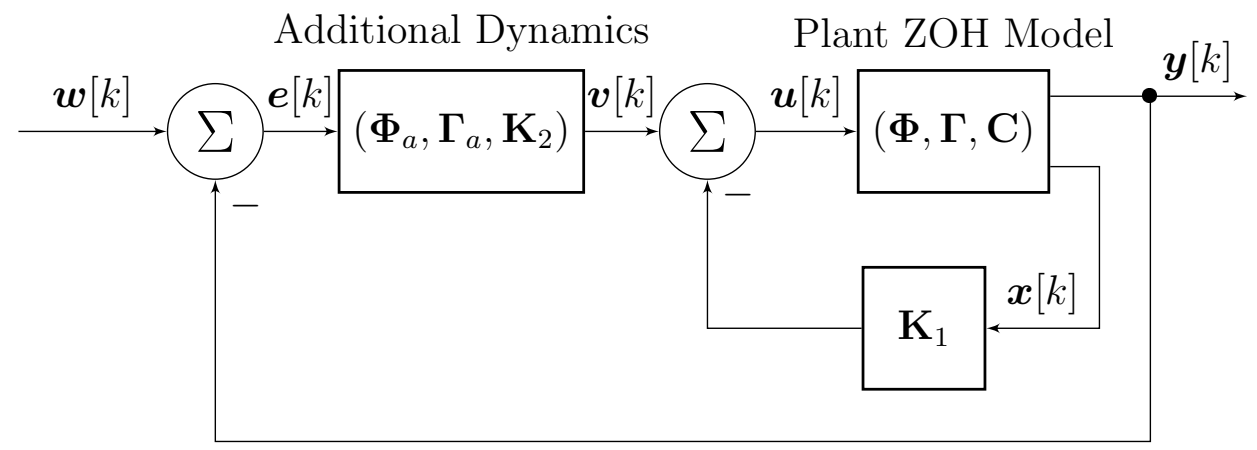

Figure 3.1: A digital state-space tracking system

where

$$
\boldsymbol{\Phi}=e^{\mathbf{A} T} \quad \boldsymbol{\Gamma}=\int_{0}^{T} e^{\mathbf{A} \tau} \mathbf{B} d \tau .
$$

For the design of a control system in which $\boldsymbol{y}[k]$ tracks a class of reference signals $\{\boldsymbol{w}[k]\}$ with zero steady-state error, we must use additional dynamics as shown in Fig. 3.1, where the poles of $\boldsymbol{\Phi}_{a}$ are the poles of the $z$-transform of $\boldsymbol{w}[k]$ [26]. The additional dynamics have the following form

$$
\begin{aligned}
\boldsymbol{x}_{a}[k+1] & =\boldsymbol{\Phi}_{a} \boldsymbol{x}_{a}[k]+\boldsymbol{\Gamma}_{a} \boldsymbol{e}[k] \\
\boldsymbol{v}[k] & =\mathbf{K}_{2} \boldsymbol{x}_{a}[k]
\end{aligned}
$$

where $\boldsymbol{e}[k]=\boldsymbol{w}[k]-\boldsymbol{y}[k]$.

Tracking step signals requires an eigenvalue at $z=1$, which gives digital integral control. For MIMO tracking systems, the additional dynamic poles have to be replicated up to the number of plant outputs, i.e. to track step signals for a plant with $p$ outputs the additional dynamics consist of a parallel combination of $p$ digital integrators:

$$
\mathbf{\Phi}_{a}=\mathbf{I}_{p} \quad \boldsymbol{\Gamma}_{a}=\mathbf{I}_{p} .
$$

In order to compute the feedback gain $\mathbf{K}_{1}$ and the integrator gain matrix $\mathbf{K}_{2}$ 
in Fig. 3.1, a design model is needed. The equations

$$
\begin{aligned}
\boldsymbol{x}[k+1] & =\boldsymbol{\Phi} \boldsymbol{x}[k]+\boldsymbol{\Gamma} \boldsymbol{u}[k] & \boldsymbol{x}_{a}[k+1] & =\boldsymbol{\Phi}_{a} \boldsymbol{x}_{a}[k]+\boldsymbol{\Gamma}_{a} \boldsymbol{e}[k] \\
\boldsymbol{y}[k] & =\mathbf{C} \boldsymbol{x}[k] & \boldsymbol{v}[k] & =\mathbf{K}_{2} \boldsymbol{x}_{a}[k] \\
\boldsymbol{u}[k] & =\boldsymbol{v}[k]-\mathbf{K}_{1} \boldsymbol{x}[k] & \boldsymbol{e}[k] & =\boldsymbol{w}[k]-\boldsymbol{y}[k]
\end{aligned}
$$

are used to form the system

$$
\begin{aligned}
{\left[\begin{array}{c}
\boldsymbol{x}[k+1] \\
\boldsymbol{x}_{a}[k+1]
\end{array}\right] } & =\left[\begin{array}{cc}
\boldsymbol{\Phi}-\boldsymbol{\Gamma} \mathbf{K}_{1} & \boldsymbol{\Gamma} \mathbf{K}_{2} \\
-\boldsymbol{\Gamma}_{a} \mathbf{C} & \boldsymbol{\Phi}_{a}
\end{array}\right]\left[\begin{array}{c}
\boldsymbol{x}[k] \\
\boldsymbol{x}_{a}[k]
\end{array}\right]+\left[\begin{array}{c}
\mathbf{0} \\
\boldsymbol{\Gamma}_{a}
\end{array}\right] \boldsymbol{w}[k] \\
& =\left(\left[\begin{array}{cc}
\boldsymbol{\Phi} & \mathbf{0} \\
-\boldsymbol{\Gamma}_{a} \mathbf{C} & \boldsymbol{\Phi}_{a}
\end{array}\right]-\left[\begin{array}{c}
\boldsymbol{\Gamma} \\
\mathbf{0}
\end{array}\right]\left[\begin{array}{ll}
\mathbf{K}_{1} & -\mathbf{K}_{2}
\end{array}\right]\right)\left[\begin{array}{c}
\boldsymbol{x}[k] \\
\boldsymbol{x}_{a}[k]
\end{array}\right]+\left[\begin{array}{c}
\mathbf{0} \\
\boldsymbol{\Gamma}_{a}
\end{array}\right] \boldsymbol{w}[k] .
\end{aligned}
$$

With the definitions

$$
\boldsymbol{\Phi}_{d}=\left[\begin{array}{cc}
\boldsymbol{\Phi} & \mathbf{0} \\
-\boldsymbol{\Gamma}_{a} \mathbf{C} & \boldsymbol{\Phi}_{a}
\end{array}\right], \quad \boldsymbol{\Gamma}_{d}=\left[\begin{array}{l}
\boldsymbol{\Gamma} \\
\mathbf{0}
\end{array}\right], \quad \mathbf{K}_{d}=\left[\begin{array}{ll}
\mathbf{K}_{1} & -\mathbf{K}_{2}
\end{array}\right]
$$

it becomes clear that $\mathbf{K}_{1}$ and $\mathbf{K}_{2}$ can be found after a feedback control design on the design model (3.8) has been performed (with an according partition of $\mathbf{K}_{d}$ ). There are several possibilities for the design of the feedback matrix $\mathbf{K}_{d}$. Given a desired set of $n+n_{a}$ desired closed-loop poles, where $n_{a}$ is the order of the additional dynamics, a $m \times\left(n+n_{a}\right)$ feedback gain matrix $\mathbf{K}_{d}$ is computed such that the eigenvalues of $\boldsymbol{\Phi}_{d}-\boldsymbol{\Gamma}_{d} \mathbf{K}_{d}$ coincide with the desired closed-loop poles. In principle, any pole-placement algorithm could be used to calculate $\mathbf{K}_{d}$. For a MIMO system, however, there are an infinite number of matrices $\mathbf{K}_{d}$ that lead to the desired closedloop pole locations. Therefore, a robust pole-placement algorithm is recommended ([27]), which finds the feedback gain matrix that maximizes a combination of the input-multiplicative $\left(\delta_{1}\right)$ and the input-feedback $\left(\delta_{2}\right)$ stability robustness bounds. The algorithm described in [27] returns the optimal $\delta_{1}$, but it was slightly modified in this thesis so that it optimizes a combination of both bounds (a weighted measure for $\delta_{2}$ was added to the cost function).

The two robustness bounds will be shortly introduced. For $\delta_{1}$, an inputmultiplicative plant pertubation model, shown in Fig 3.2a, is considered [28]. Let 
the system from $\boldsymbol{w}[k]$ to $\boldsymbol{v}[k]$, when all external inputs are set to zero, be denoted by $\mathbf{H}_{1}(z)$. The result is shown is Fig. $3.2 \mathrm{~b}$. Note that $\mathbf{H}_{1}(z)$ is stable, since it has the same poles as the nominal closed-loop control system that is stable by design [28]. If the plant is a $p$-input system, then $\boldsymbol{\Delta}_{1}$ is a $p$-input, $p$-output system which is assumed to be stable. It represents a perturbation to the nominal plant model. The small gain theorem is used to examine the stability of the system shown in Fig. 3.2. It gives a sufficient condition for stability for the feedback interconnection of two stable systems, as shown in Fig. 3.2b. It states states that the closed-loop system is guaranteed to be stable if

$$
\left\|\mathbf{H}_{1}(z)\right\|_{\infty}\left\|\boldsymbol{\Delta}_{1}(z)\right\|_{\infty}<1
$$

holds, where $\|\cdot\|_{\infty}$ denotes the system infinity norm [28]. This inequality may be rewritten to obtain a bound on the size of the unknown pertubation system:

$$
\left\|\boldsymbol{\Delta}_{1}(z)\right\|_{\infty}<\frac{1}{\left\|\mathbf{H}_{1}(z)\right\|_{\infty}}=: \delta_{1}
$$

The right-hand size of this inequality is defined as the input-multiplicative stability robustness bound $\delta_{1}[28]$. For the feedback tracking system in Fig. 3.1, the system $\mathbf{H}_{1}(z)$ is given as the following state-space system (note that $\boldsymbol{w}[k]$ refers to the notation in Fig. 3.2 here, and not to Fig. 3.1):

$$
\begin{aligned}
{\left[\begin{array}{c}
\boldsymbol{x}[k+1] \\
\boldsymbol{x}_{a}[k+1]
\end{array}\right] } & =\left[\begin{array}{cc}
\boldsymbol{\Phi}-\boldsymbol{\Gamma} \mathbf{K}_{1} & \boldsymbol{\Gamma} \mathbf{K}_{2} \\
-\boldsymbol{\Gamma}_{a} \mathbf{C} & \boldsymbol{\Phi}_{a}
\end{array}\right]\left[\begin{array}{c}
\boldsymbol{x}[k] \\
\boldsymbol{x}_{a}[k]
\end{array}\right]+\left[\begin{array}{c}
\boldsymbol{\Gamma} \\
\mathbf{0}
\end{array}\right] \boldsymbol{w}[k] \\
\boldsymbol{v}[k] & =\left[\begin{array}{ll}
-\mathbf{K}_{1} & \mathbf{K}_{2}
\end{array}\right]\left[\begin{array}{c}
\boldsymbol{x}[k] \\
\boldsymbol{x}_{a}[k]
\end{array}\right]
\end{aligned}
$$

With this system description, $\delta_{1}$ can be computed as the reciprocal of the system infinity norm.

The input-feedback stability robustness bound $\left(\delta_{2}\right)$ is defined in a similar way. The difference to $\delta_{1}$ is that a input-feedback plant perturbation model, shown in Fig. 3.3, is considered [28]. The procedure to determine $\delta_{2}$ is the same, i.e. find 


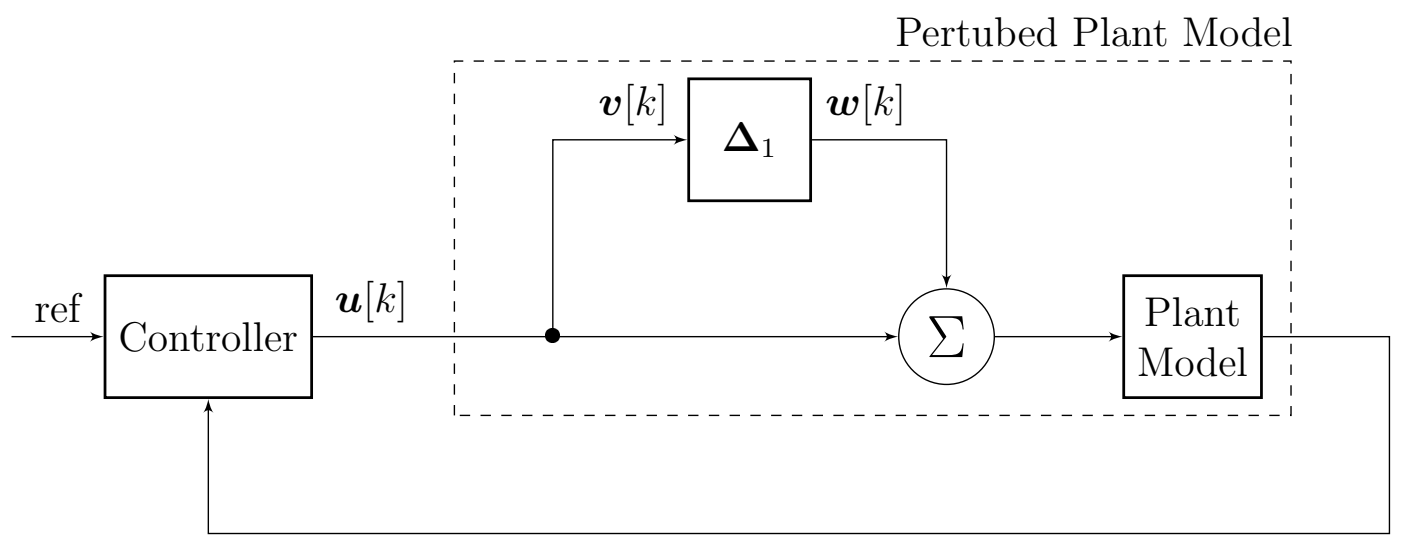

(a) Input-muliplicative plant pertubarion model

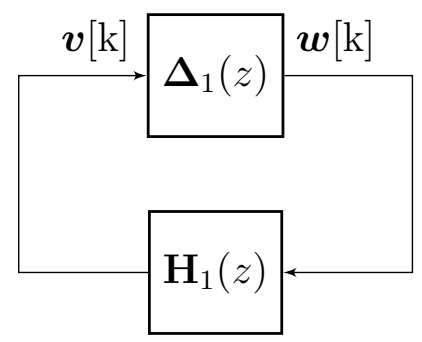

(b) Closed-loop system for input-muliplicative plant pertubarion model when all external inputs are set to zero

Figure 3.2: Input-multiplicative stability robustness bound 


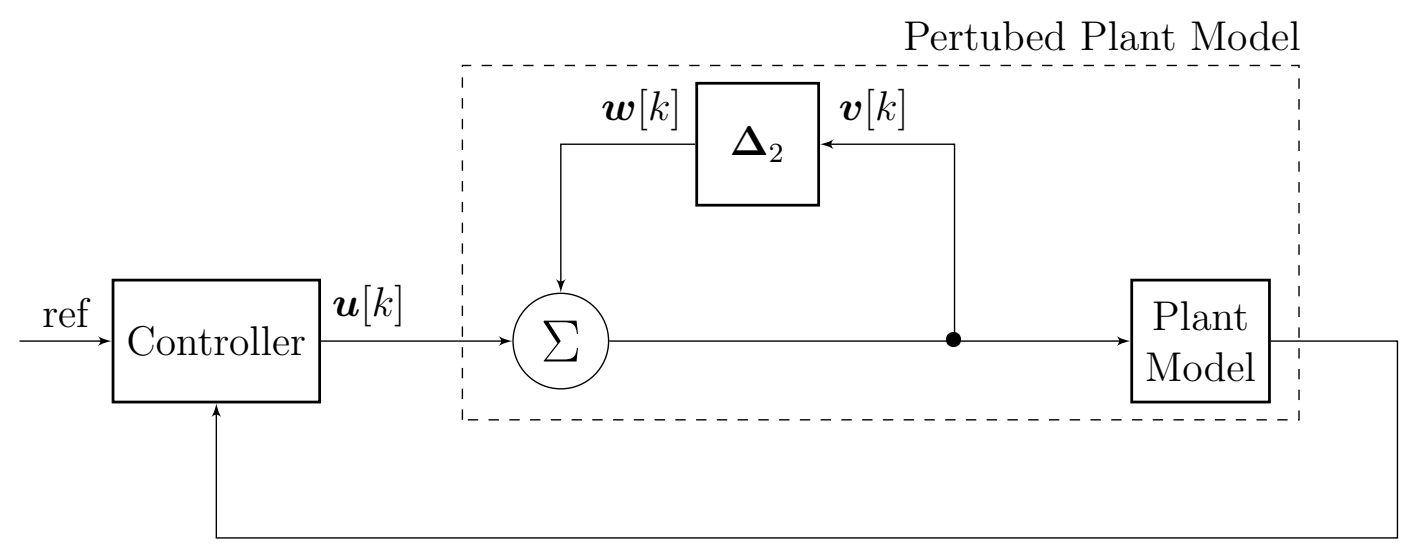

Figure 3.3: Input-feedback plant perturbation model

the system from $\boldsymbol{w}[k]$ to $\boldsymbol{v}[k]$, called $\mathbf{H}_{2}(z)$, and use the small gain theorem to determine the stability of the interconnection of $\mathbf{H}_{2}$ and $\boldsymbol{\Delta}_{2}$. The result is

$$
\delta_{2}:=\frac{1}{\left\|\mathbf{H}_{2}(z)\right\|_{\infty}}
$$

The system $\mathbf{H}_{2}(z)$ for the feedback tracking system in Fig. 3.1 is given by:

$$
\begin{aligned}
{\left[\begin{array}{c}
\boldsymbol{x}[k+1] \\
\boldsymbol{x}_{a}[k+1]
\end{array}\right] } & =\left[\begin{array}{cc}
\boldsymbol{\Phi}-\boldsymbol{\Gamma} \mathbf{K}_{1} & \boldsymbol{\Gamma} \boldsymbol{K}_{2} \\
-\boldsymbol{\Gamma}_{a} \mathbf{C} & \boldsymbol{\Phi}_{a}
\end{array}\right]\left[\begin{array}{c}
\boldsymbol{x}[k] \\
\boldsymbol{x}_{a}[k]
\end{array}\right]+\left[\begin{array}{c}
\boldsymbol{\Gamma} \\
\mathbf{0}
\end{array}\right] \boldsymbol{w}[k] \\
\boldsymbol{v}[k] & =\left[\begin{array}{ll}
-\mathbf{K}_{1} & \mathbf{K}_{2}
\end{array}\right]\left[\begin{array}{c}
\boldsymbol{x}[k] \\
\boldsymbol{x}_{a}[k]
\end{array}\right]+\mathbf{I}_{p} \boldsymbol{w}[k] .
\end{aligned}
$$

The larger the bound $\delta_{1}$ is, the more tolerant the control system is to errors in the plant model, the same goes for $\delta_{2}$. As a rule of thumb, a control system with $\delta_{1}>0.5$ is desired [28].

Recommendations for the pole locations of feedback tracking systems can also be found in [27]. Another possibility for the feedback design is to use the discretetime linear quadratic regulator $(d l q r)$ formulas.

In the next sections, the derivations of filters is given which, by cascading them with the closed-loop system in Fig. 3.1, increase the precision-tracking bandwidth.

\subsection{Algorithm for Stable System Inversion}

In this section, the general procedure to invert a stable digital system, with input $\boldsymbol{w}[k]$ and output $\boldsymbol{y}[k]$, is presented $[24,25]$. Let the $n_{c}$ th order, $p$ input and 
$p$ output, system be given by its state-space representation

$$
\begin{aligned}
\boldsymbol{x}_{c}[k+1] & =\boldsymbol{\Phi}_{c} \boldsymbol{x}_{c}[k]+\boldsymbol{\Gamma}_{c} \boldsymbol{w}[k] \\
\boldsymbol{y}[k] & =\mathbf{C}_{c} \boldsymbol{x}_{c}[k] .
\end{aligned}
$$

The goal of the following filter design is to invert the model of the system (3.14). Consider $r$ advances of the plant output, $\boldsymbol{y}[k+r]$. Let $r$ be the smallest integer for which $\mathbf{C}_{c} \boldsymbol{\Phi}_{c}^{r-1} \boldsymbol{\Gamma}_{c}$ is a nonzero matrix. It follows that

$$
\boldsymbol{y}[k+r]=\mathbf{C}_{c} \boldsymbol{\Phi}_{c}^{r} \boldsymbol{x}_{c}[k]+\mathbf{C}_{c} \boldsymbol{\Phi}_{c}^{r-1} \boldsymbol{\Gamma}_{c} \boldsymbol{w}[k]
$$

holds. This result can be easily shown:

$$
\begin{gathered}
\boldsymbol{y}[k+1]=\mathbf{C}_{c} \boldsymbol{x}_{c}[k+1]=\mathbf{C}_{c} \boldsymbol{\Phi}_{c} \boldsymbol{x}_{c}[k]+\underbrace{\mathbf{C}_{c} \boldsymbol{\Gamma}_{c}}_{=\mathbf{0}} \boldsymbol{w}[k] \\
\boldsymbol{y}[k+2]=\mathbf{C}_{c} \boldsymbol{\Phi}_{c} \boldsymbol{x}_{c}[k+1]=\mathbf{C}_{c} \boldsymbol{\Phi}_{c}^{2} \boldsymbol{x}_{c}[k]+\underbrace{\mathbf{C}_{c} \boldsymbol{\Phi}_{\mathbf{c}} \boldsymbol{\Gamma}_{c}}_{=\mathbf{0}} \boldsymbol{w}[k] \\
\vdots \\
\boldsymbol{y}[k+m]=\mathbf{C}_{c} \boldsymbol{\Phi}_{c}^{m-1} \boldsymbol{x}_{c}[k+1]=\mathbf{C}_{c} \boldsymbol{\Phi}_{c}^{m} \boldsymbol{x}_{c}[k]+\underbrace{\mathbf{C}_{c} \boldsymbol{\Phi}_{c}^{m-1} \boldsymbol{\Gamma}_{c}}_{=\mathbf{0}} \boldsymbol{w}[k] \\
\vdots \\
\boldsymbol{y}[k+r]=\mathbf{C}_{c} \boldsymbol{\Phi}_{c}^{r-1} \boldsymbol{x}_{c}[k+1]=\mathbf{C}_{c} \boldsymbol{\Phi}_{c}^{r} \boldsymbol{x}_{c}[k]+\underbrace{\mathbf{C}_{c} \boldsymbol{\Phi}_{c}^{r-1} \boldsymbol{\Gamma}_{c}}_{\neq \mathbf{0}} \boldsymbol{w}[k]
\end{gathered}
$$

with $m<r$. Define

$$
\widetilde{\mathbf{D}}_{f}=\left(\mathbf{C}_{c} \boldsymbol{\Phi}_{c}^{r-1} \boldsymbol{\Gamma}_{c}\right)^{-1}
$$

and rearrange $(3.15)$

$$
\boldsymbol{w}[k]=-\widetilde{\mathbf{D}}_{f} \mathbf{C}_{c} \boldsymbol{\Phi}_{c}^{r} \boldsymbol{x}_{c}[k]+\widetilde{\mathbf{D}}_{f} \boldsymbol{y}[k+r]
$$

to obtain the ideal inversion control law $\boldsymbol{w}[k]$. Substituting this equation into (3.14a) yields

$$
\begin{aligned}
\boldsymbol{x}_{c}[k+1] & =\boldsymbol{\Phi}_{c} \boldsymbol{x}_{c}+\boldsymbol{\Gamma}_{c}\left(-\widetilde{\mathbf{D}}_{f} \mathbf{C}_{c} \boldsymbol{\Phi}_{c}^{r} \boldsymbol{x}_{c}[k]+\widetilde{\mathbf{D}}_{f} \boldsymbol{y}[k+r]\right) \\
& =\left(\boldsymbol{\Phi}_{c}-\boldsymbol{\Gamma}_{c} \widetilde{\mathbf{D}}_{f} \mathbf{C}_{c} \boldsymbol{\Phi}_{c}^{r}\right) \boldsymbol{x}_{c}[k]+\boldsymbol{\Gamma}_{c} \widetilde{\mathbf{D}}_{f} \boldsymbol{y}[k+r]
\end{aligned}
$$


Equations (3.19) and (3.18) show that a system

$$
\begin{aligned}
\boldsymbol{x}_{f}[k+1] & =\widetilde{\boldsymbol{\Phi}}_{f} \boldsymbol{x}_{f}[k]+\widetilde{\boldsymbol{\Gamma}}_{f} \boldsymbol{y}[k+r] \\
\boldsymbol{w}[k] & =\widetilde{\mathbf{C}}_{f} \boldsymbol{x}_{f}[k]+\widetilde{\mathbf{D}}_{f} \boldsymbol{y}[k+r]
\end{aligned}
$$

where $\widetilde{\mathbf{D}}_{f}$ is given by (3.17) and where

$$
\begin{aligned}
\widetilde{\mathbf{C}}_{f} & =-\widetilde{\mathbf{D}}_{f} \mathbf{C}_{c} \boldsymbol{\Phi}_{c}^{r} \\
\widetilde{\boldsymbol{\Gamma}}_{f} & =\boldsymbol{\Gamma}_{c} \widetilde{\mathbf{D}}_{f} \\
\widetilde{\boldsymbol{\Phi}}_{f} & =\boldsymbol{\Phi}_{c}+\boldsymbol{\Gamma}_{c} \widetilde{\mathbf{C}}_{f}
\end{aligned}
$$

holds may be used as a feedforward filter to invert the system (3.14), since it produces the signal $\boldsymbol{w}[k]$, which inverts the system model (3.14) with a delay of $r$ samples, from the advanced plant output $\boldsymbol{y}[k+r]$. That is, the cascade of the filter (3.20) and the system (3.14) is a pure delay of $r$ samples on each output signal. Thus, the filter in (3.20) is theoretically able to achieve perfect tracking with a delay of $r$ samples.

However, the eigenvalues of the filter will include the zeros of the system under consideration (see Section 3.2.1). Therefore, if the system (3.14) is non-minimum phase (either because the continuous-time system is already non-minimum phase or due to the presence of unstable "sampling zeros"), the filter in (3.20) cannot be used as a feedforward filter, since it is an unstable system. Additional calculations are needed to obtain a stable approximate inverse of the system.

\subsubsection{Eigenvalues of the Inverse Filter}

This section shows why the system (3.20), which inverts the system (3.14) with a delay of $r$ samples, cannot be used as a feedforward filter for non-minimum phase systems (see also [9]). As mentioned before, this is due to the fact that it contains the zeros of system (3.14) as eigenvalues. 
First, we will make an assumptions about system (3.14): we assume that it is minimal, because then the transmission zeros of the system coincide with its invariant zeros [29].

The transmission zeros of a system with transmission matrix $\mathbf{G}(z)$ are defined as the values $\eta_{i}$ for which

$$
\operatorname{rank}\left(\mathbf{G}\left(\eta_{i}\right)\right)<\max _{z} \operatorname{rank}(\mathbf{G}(z))
$$

holds [30]. For systems where the number of inputs is equal to the number of outputs, this condition reduces to

$$
\operatorname{det}\left(\mathbf{G}\left(\eta_{i}\right)\right)=0
$$

It is noted that other definitions for the transmission zeros exist in the literature, they can also be defined with the help of the Smith-McMillan form [31, 32]. Particularly in [32] it is mentioned that (3.23) "can not in general be used to find or define the zeros and poles of a square matrix $\mathbf{G}(s)$ ". This is due to the fact that in

$$
\operatorname{det}(\mathbf{G}(s))=\alpha \frac{z(s)}{p(s)}
$$

the polynomials $z(s)$ and $p(s)$ "are not necessarily relatively prime" [32]. In this thesis, however, it is assumed that the definition for the transmission zeros in (3.23) and the definition with the Smith-McMillan form coincide because we consider only minimal state-space models.

The invariant zeros are defined with the help of the Rosenbrock matrix $\mathbf{P}(z)$ $[30,31,32]$. The Rosenbrock matrix for a system $(\mathbf{A}, \mathbf{B}, \mathbf{C}, \mathbf{D})$ is defined as

$$
\mathbf{P}(z)=\left[\begin{array}{cc}
z \mathbf{I}-\mathbf{A} & -\mathbf{B} \\
\mathbf{C} & \mathbf{D}
\end{array}\right] .
$$

The invariant zeros are the values $\eta_{i}$ for which the Rosenbrock matrix is rankdeficient:

$$
\operatorname{rank}\left(\mathbf{P}\left(\eta_{i}\right)\right)<\max _{z} \operatorname{rank}(\mathbf{P}(z))
$$


Again, for a system with the same number of inputs and outputs, this reduces to

$$
\operatorname{det}\left(\mathbf{P}\left(\eta_{i}\right)\right)=0 .
$$

Let the transmission matrix of system (3.14) be denoted by $\mathbf{G}(z)$ :

$$
\mathbf{G}(z)=\mathbf{C}_{c}\left(z \mathbf{I}-\boldsymbol{\Phi}_{c}\right)^{-1} \boldsymbol{\Gamma}_{c} .
$$

Therefore, its transmission zeros can be found according to (3.23). We now consider the same system, but we assume that the output is $\boldsymbol{y}[k+r]$, and not $\boldsymbol{y}[k]$. The transfer function of this system is given by

$$
\widetilde{\mathbf{G}}(z)=\frac{\mathcal{Z}\{y[k+r]\}}{\mathcal{Z}\{w[k]\}}=z^{r} \underbrace{\frac{\mathcal{Z}\{y[k]\}}{\mathcal{Z}\{w[k]\}}}_{=\mathbf{G}(z)}=z^{r} \mathbf{G}(z)
$$

where $\mathcal{Z}\{\cdot\}$ denotes the $z$-transform and the shifting property of the $z$-transform was used (see for example $[26,33])$. Thus, the transmission zeros of system (3.14), where the output is advanced by $r$ samples, can be found by

$$
\operatorname{det}(\widetilde{\mathbf{G}}(z))=\operatorname{det}\left(z^{r} \mathbf{G}(z)\right)=z^{p \cdot r} \operatorname{det}(\mathbf{G}(z))=0 .
$$

For this computation, the fact that for a square $n \times n$ matrix $\mathbf{A}$

$$
\operatorname{det}(\lambda \mathbf{A})=\lambda^{n} \operatorname{det}(\mathbf{A})
$$

holds, was used [26]. It follows from (3.30) that the system with the advanced output has the same transmission zeros as the original system, but additionally it has $p \cdot r$ transmission zeros in 0 .

The state-space representation of the system, when the output is advanced by $r$ samples, is given by

$$
\begin{aligned}
\boldsymbol{x}_{c}[k+1] & =\boldsymbol{\Phi}_{c} \boldsymbol{x}_{c}[k]+\boldsymbol{\Gamma}_{c} \boldsymbol{w}[k] \\
\boldsymbol{y}[k+r] & =\mathbf{C}_{c} \boldsymbol{\Phi}_{c}^{r} \boldsymbol{x}_{c}[k]+\mathbf{C}_{c} \boldsymbol{\Phi}_{c}^{r-1} \boldsymbol{\Gamma}_{c} \boldsymbol{w}[k] .
\end{aligned}
$$


Due to our assumption, the transmission zeros of the system coincide with its invariant zeros, so the Rosenbrock matrix can be used as well to find the transmission zeros. The Rosenbrock matrix for the system (3.32) is defined as

$$
\mathbf{P}(z)=\left[\begin{array}{cc}
z \mathbf{I}-\boldsymbol{\Phi}_{c} & -\boldsymbol{\Gamma}_{c} \\
\mathbf{C}_{c} \boldsymbol{\Phi}_{c}^{r} & \mathbf{C}_{c} \boldsymbol{\Phi}_{c}^{r-1} \boldsymbol{\Gamma}_{c}
\end{array}\right]
$$

and for every invariant zero $\eta_{i}$ of system (3.32), this matrix is rank-deficient. Each invariant zero $\eta_{i}$ is associated with an invariant-zero direction $\boldsymbol{z}_{0}=\left[\begin{array}{l}\boldsymbol{z}_{x 0} \\ \boldsymbol{z}_{w 0}\end{array}\right]$ which lies in the kernel or null space of $\mathbf{P}\left(\eta_{i}\right)[32]$ :

$$
\left[\begin{array}{cc}
\eta_{i} \mathbf{I}-\boldsymbol{\Phi}_{c} & -\boldsymbol{\Gamma}_{c} \\
\mathbf{C}_{c} \boldsymbol{\Phi}_{c}^{r} & \mathbf{C}_{c} \boldsymbol{\Phi}_{c}^{r-1} \boldsymbol{\Gamma}_{c}
\end{array}\right]\left[\begin{array}{l}
\boldsymbol{z}_{x 0} \\
\boldsymbol{z}_{w 0}
\end{array}\right]=\mathbf{0} .
$$

From the definition of the zero direction, the two equations

$$
\begin{array}{r}
\left(\eta_{i} \mathbf{I}-\boldsymbol{\Phi}_{c}\right) \boldsymbol{z}_{x 0}-\boldsymbol{\Gamma}_{c} \boldsymbol{z}_{w 0}=0 \\
\mathbf{C}_{c} \boldsymbol{\Phi}_{c}^{r} \boldsymbol{z}_{x 0}+\mathbf{C}_{c} \boldsymbol{\Phi}_{c}^{r-1} \boldsymbol{\Gamma}_{c} \boldsymbol{z}_{w 0}=0
\end{array}
$$

follow. Equation (3.35b) can be rearranged

$$
\boldsymbol{z}_{w 0}=\overbrace{-\underbrace{\left(\mathbf{C}_{c} \boldsymbol{\Phi}_{c}^{r-1} \boldsymbol{\Gamma}_{c}\right)^{-1}}_{=\widetilde{\mathbf{D}}_{f}} \mathbf{C}_{c} \boldsymbol{\Phi}_{c}^{r}}^{=\widetilde{\mathbf{C}}_{f}} \boldsymbol{z}_{x 0}=\widetilde{\mathbf{C}}_{f} \boldsymbol{z}_{x 0}
$$

where the definitions (3.18) and (3.21a) have been used. This result is then plugged into (3.35a):

$$
\left(\eta_{i} \mathbf{I}-(\underbrace{\boldsymbol{\Phi}_{c}+\boldsymbol{\Gamma}_{c} \widetilde{\mathbf{C}}_{f}}_{=\widetilde{\boldsymbol{\Phi}}_{f}})\right) \boldsymbol{z}_{x 0}=\left(\eta_{i} \mathbf{I}-\widetilde{\boldsymbol{\Phi}}_{f}\right) \boldsymbol{z}_{x 0}=0
$$

so it becomes clear that the invariant zeros $\eta_{i}$ of system (3.32) are the eigenvalues of the matrix $\widetilde{\boldsymbol{\Phi}}_{f}=\boldsymbol{\Phi}_{c}+\boldsymbol{\Gamma}_{c} \widetilde{\mathbf{C}}_{f}$ defined in (3.21c), but this is also the state-transition matrix of the inverse filter. Together with the previous result from this section, it can be concluded that the eigenvalues of the inverse filter in (3.20) consist of the invariant zeros of system (3.14) (this includes possible non-minimum phase zeros) and $p \cdot r$ eigenvalues at 0 . Consequently, more steps have to be taken in order to stabilize the inverse filter (3.20), which will be discussed in the following section. 


\subsubsection{Adding Additional Advances}

Now, the stabilization of the filter (3.20) by adding more advances is shown. Consider adding $s$ more advances to the $r$ advances in (3.15). Let $d=r+s$. Then it follows:

$$
\boldsymbol{y}[k+d]=\mathbf{C}_{c} \boldsymbol{\Phi}_{c}^{d} \boldsymbol{x}_{c}[k]+\sum_{i=0}^{s} \mathbf{C}_{c} \boldsymbol{\Phi}_{c}^{d-i-1} \boldsymbol{\Gamma}_{c} \boldsymbol{w}[k+i] .
$$

With the approximation

$$
\boldsymbol{w}[k+i] \approx \boldsymbol{w}[k], \quad i=1, \ldots, s
$$

which will hold true for low frequency signals, equation (3.38) becomes

$$
\boldsymbol{y}[k+d] \approx \mathbf{C}_{c} \boldsymbol{\Phi}_{c}^{d} \boldsymbol{x}_{c}[k]+\left(\sum_{i=0}^{s} \mathbf{C}_{c} \boldsymbol{\Phi}_{c}^{d-i-1} \boldsymbol{\Gamma}_{c}\right) \boldsymbol{w}[k] .
$$

Define

$$
\mathbf{D}_{f}=\left(\sum_{i=0}^{s} \mathbf{C}_{c} \boldsymbol{\Phi}_{c}^{d-i-1} \boldsymbol{\Gamma}_{c}\right)^{-1}
$$

Now the same procedure as before is used, i.e. solving (3.40) for $\boldsymbol{w}[k]$ and substituting it into (3.14a), so the new filter $\left(\boldsymbol{\Phi}_{f}, \boldsymbol{\Gamma}_{f}, \mathbf{C}_{f}, \mathbf{D}_{f}\right)$ is obtained, where $\mathbf{D}_{f}$ is defined according to (3.41) and

$$
\begin{aligned}
\mathbf{C}_{f} & =-\mathbf{D}_{f} \mathbf{C}_{c} \boldsymbol{\Phi}_{c}^{d} \\
\boldsymbol{\Gamma}_{f} & =\boldsymbol{\Gamma}_{c} \mathbf{D}_{f} \\
\boldsymbol{\Phi}_{f} & =\boldsymbol{\Phi}_{c}+\boldsymbol{\Gamma}_{c} \mathbf{C}_{f}
\end{aligned}
$$

holds. The advantage of adding more delays is that the new filter (3.42) will be stable for a sufficiently large $d$. The state-transition matrix is $\boldsymbol{\Phi}_{f}=\boldsymbol{\Phi}_{c}+\boldsymbol{\Gamma}_{c} \mathbf{C}_{f}$ and the entries of $\mathbf{C}_{f}=-\mathbf{D}_{f} \mathbf{C}_{c} \boldsymbol{\Phi}_{c}^{d}$ can be made arbitrarily small by increasing $d$, so the eigenvalues of $\boldsymbol{\Phi}_{f}$ will move towards the eigenvalues of $\boldsymbol{\Phi}_{c}$, from which we assume that it is stable. 
This result can be verified using the Bauer/Fike Theorem [34]. Let $\mathbf{A} \in \mathbb{C}^{n \times n}$ be a diagonalizable matrix with eigenvalues $\lambda_{i}, i=1, \ldots, n$, so

$$
\mathbf{T}^{-1} \mathbf{A} \mathbf{T}=\operatorname{diag}\left(\lambda_{1}, \ldots, \lambda_{n}\right)
$$

Assume that $\mu$ is an eigenvalue of the perturbed matrix $\mathbf{A}+\Delta \mathbf{A}$, with $\Delta \mathbf{A} \in \mathbb{C}^{n \times n}$. Then

$$
\min _{i=1, \ldots, n}\left|\mu-\lambda_{i}\right| \leq \operatorname{cond}_{2}(\mathbf{T})\|\Delta \mathbf{A}\|_{2}
$$

holds, i.e. $\mu$ is within a circle with radius $\operatorname{cond}_{2}(\mathbf{T})\|\Delta \mathbf{A}\|_{2}$ centered on an eigenvalue of $\mathbf{A}$. Here, $\|\cdot\|_{2}$ denotes the matrix norm induced by the Euclidean norm, and $\operatorname{cond}_{2}(\mathbf{T})=\|\mathbf{T}\|_{2}\left\|\mathbf{T}^{-1}\right\|_{2}$ is the condition number of the transformation matrix $\mathbf{T}$. From this result, it follows that the eigenvalues of $\boldsymbol{\Phi}_{f}$ are within a circle that is centered in an eigenvalue of $\boldsymbol{\Phi}_{c}$, and the radius of this circle depends on the matrix norm of $\boldsymbol{\Gamma}_{c} \mathbf{C}_{f}$ (and also on how "well" $\boldsymbol{\Phi}_{c}$ can be diagonalized), since we have $\mathbf{A}=\boldsymbol{\Phi}_{c}$ and $\Delta \mathbf{A}=\boldsymbol{\Gamma}_{c} \mathbf{C}_{f}$. Accordingly, the inequality

$$
\min _{i=1, \ldots, n}\left|\mu-\lambda_{i}\right| \leq \operatorname{cond}_{2}(\mathbf{T})\left\|\boldsymbol{\Gamma}_{c} \mathbf{C}_{f}\right\|_{2}
$$

follows. Since any matrix norm induced by a vector norm is submultiplicative $[34,35]$, we can further write, together with (3.42a):

$$
\begin{aligned}
\left\|\boldsymbol{\Gamma}_{c} \mathbf{C}_{f}\right\|_{2} & =\left\|-\boldsymbol{\Gamma}_{c} \mathbf{D}_{f} \mathbf{C}_{c} \boldsymbol{\Phi}_{c}^{d}\right\|_{s}=\left\|\boldsymbol{\Gamma}_{c} \mathbf{D}_{f} \mathbf{C}_{c} \boldsymbol{\Phi}_{c}^{d}\right\|_{2} \\
& \leq\left\|\boldsymbol{\Gamma}_{c} \mathbf{D}_{f} \mathbf{C}_{c}\right\|_{2}\left\|\boldsymbol{\Phi}_{c}^{d}\right\|_{2} \leq K_{2}\left\|\mathbf{D}_{f}\right\|_{2}\left\|\boldsymbol{\Phi}_{c}^{d}\right\|_{2}
\end{aligned}
$$

where $K_{2}$ has been defined as $K_{2}=\left\|\boldsymbol{\Gamma}_{c}\right\|_{2}\left\|\mathbf{C}_{c}\right\|_{2}$. The 2-norm of a matrix can also be expressed as its maximum singular value [34], and it can be shown that

$$
\left\|\mathbf{A}^{-1}\right\|_{2}=\sigma_{\max }\left(\mathbf{A}^{-1}\right)=\frac{1}{\sigma_{\min }(\mathbf{A})}
$$

holds [36]. Here, $\sigma_{\min }(\cdot)$ denotes the minimum singular value of a matrix. In the light of this result, it becomes clear that

$$
\frac{\left\|\boldsymbol{\Phi}_{c}^{d}\right\|_{2}}{\sigma_{\min }\left(\sum_{i=0}^{s} \mathbf{C}_{c} \boldsymbol{\Phi}_{c}^{d-i-1} \boldsymbol{\Gamma}_{c}\right)} \stackrel{d \rightarrow \infty}{\longrightarrow} 0
$$


has to be shown, where the definition in (3.41) was used. Since we assume that $\boldsymbol{\Phi}_{c}$ is stable, we have $\left|\lambda_{i}\right|<1, i=1, \ldots, n_{c}$ for every eigenvalue of $\boldsymbol{\Phi}_{c}$, thus $\boldsymbol{\Phi}_{c}^{d}$ converges to the zero matrix as $d$ tends towards infinity [26]. This means that the numerator of (3.48) tends towards zero. It is further well known that $\sigma_{\min }>0$ holds for a full-rank matrix [26]. Because $\mathbf{D}_{f}$ is defined as the inverse of the matrix $\left(\sum_{i=0}^{s} \mathbf{C}_{c} \boldsymbol{\Phi}_{c}^{d-i-1} \boldsymbol{\Gamma}_{c}\right)$ in (3.41), we can assume that $\left(\sum_{i=0}^{s} \mathbf{C}_{c} \boldsymbol{\Phi}_{c}^{d-i-1} \boldsymbol{\Gamma}_{c}\right)$ has full rank (because otherwise the entire method is not applicable), and therefore the denominator in (3.48) is greater than zero, so $\left\|\Phi_{c}^{d}\right\|_{2}\left\|\mathbf{D}_{f}\right\|_{2}$ does converge to zero as $d$ tends towards infinity.

From (3.46), this implies that

$$
\lim _{d \rightarrow \infty}\left\|\boldsymbol{\Gamma}_{c} \mathbf{C}_{f}\right\|_{2}=0
$$

Using (3.42c), this results leads to the recognition that, as $d$ approaches infinity, the eigenvalues of $\boldsymbol{\Phi}_{f}$ and $\boldsymbol{\Phi}_{c}$ coincide. Due to the assumption that $\boldsymbol{\Phi}_{c}$ is stable, it follows that the inverse filter will eventually be stabilized when more advances $d$ are added.

Consequently, the value of $d$ is chosen to be smallest integer for which the state-transition matrix $(3.42 \mathrm{c})$ of the inverse filter is stabilized. That is

$$
d^{*}=\text { smallest } d \text { s.t. } \max \left(\operatorname{abs}\left(\operatorname{eig}\left(\boldsymbol{\Phi}_{c}-\boldsymbol{\Gamma}_{c} \mathbf{D}_{f} \mathbf{C}_{c} \boldsymbol{\Phi}_{c}^{d}\right)\right)\right)<R
$$

where $R$ is a user-defined maximum pole radius. If the assumption that $\boldsymbol{\Phi}_{c}$ is stable is fair, the choice of $R$ can be based on the eigenvalues of $\boldsymbol{\Phi}_{c}$. It is recommended to chose $R$ to be exactly in between the largest absolute value of the eigenvalues of $\boldsymbol{\Phi}_{c}$ and 1. That is, if $\lambda_{\max }$ denotes the eigenvalue with the largest absolute value, $R$ is calculated according to

$$
R=\frac{1}{2}\left(\left|\lambda_{\max }\right|+1\right) .
$$




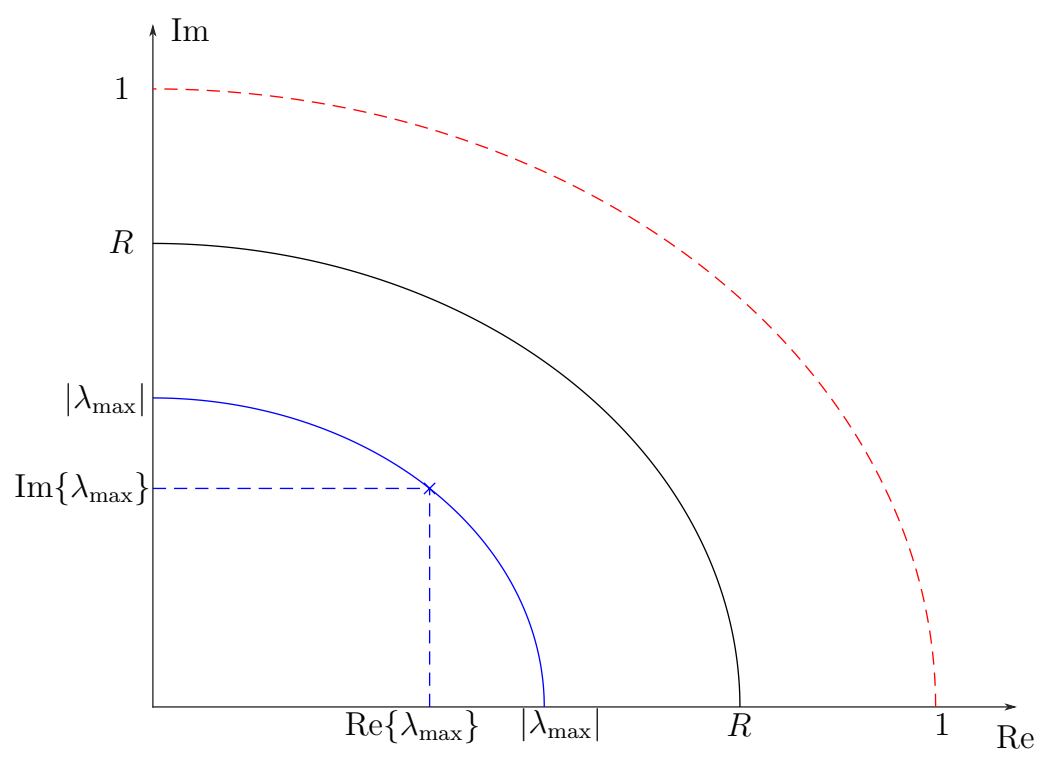

Figure 3.4: Pole radius for a stable $\boldsymbol{\Phi}_{c}$

This is illustrated in Fig. 3.4. Note that this choice of halfway was made arbitrarily, other choices (e.g. $75 \%$ of the distance from the maximum eigenvalue to the unit circle) are possible and could be investigated. The pole radius can, for example, also be chosen such that the algorithm just stabilizes the filter, e.g. $R=0.99$, without any more requirements for the absolute value of the pole locations.

Due to the approximation in (3.39), the cascade of the filter and the system will not be a pure delay of $d$ samples. The approximation is exact at dc and the error caused by the approximation will increase with increasing frequency of the signal $\boldsymbol{w}[k]$. The frequency range over which the filter provides an accurate inversion may be obtained by evaluating the Bode plot of the cascade of the filter and the system.

\subsubsection{Extension to Feedback Approach}

In this section, an alternative approach to stabilize the inverse filter (3.20), which inverts the system (3.14) with a delay of $r$ samples, is presented. In contrast 
to the algorithm shown in Section 3.2.2, this new approach does not necessarily use more advances, but uses feedback to stabilize the filter. The idea for this approach was taken from [19].

The goal is still to find an inverse filter for the system (3.14), and the first steps are exactly the same as presented in Section 3.2, i.e. consider $r$ advances of the output and rearrange (3.15) to obtain the ideal inversion control law (3.18). Next, a possibility to stabilize (3.20) is discussed. Similar to [19], an additive term $\boldsymbol{v}[k]$ is included in the optimal inversion control law (3.18)

$$
\boldsymbol{w}[k]=-\widetilde{\mathbf{D}}_{f} \mathbf{C}_{c} \boldsymbol{\Phi}_{c}^{r} \boldsymbol{x}_{c}[k]+\widetilde{\mathbf{D}}_{f} \boldsymbol{y}_{d}[k+r]+\boldsymbol{v}[k]
$$

SO

$$
\begin{aligned}
\boldsymbol{x}_{c}[k+1] & =\widetilde{\boldsymbol{\Phi}}_{f} \boldsymbol{x}_{c}[k]+\widetilde{\boldsymbol{\Gamma}}_{f} \boldsymbol{y}_{d}[k+r]+\boldsymbol{\Gamma}_{c} \boldsymbol{v}[k] \\
\boldsymbol{w}[k] & =\widetilde{\mathbf{C}}_{f} \boldsymbol{x}_{c}[k]+\widetilde{\mathbf{D}}_{f} \boldsymbol{y}_{d}[k+r]+\boldsymbol{v}[k]
\end{aligned}
$$

is obtained as the "inverse" system. This system, however, is not the exact inverse anymore, since the ideal inversion control law (3.18) was modified. Subsequently, it is determined how the new term $\boldsymbol{v}[k]$ can be chosen. If the original system $\left(\boldsymbol{\Phi}_{c}, \boldsymbol{\Gamma}_{c}\right)$ in (3.14) is controllable, the system $\left(\widetilde{\boldsymbol{\Phi}}_{f}, \boldsymbol{\Gamma}_{c}\right)$ is controllable as well. For that reason, feedback of the form

$$
\boldsymbol{v}[k]=\mathbf{F} \boldsymbol{x}_{c}[k]
$$

may be introduced to move the eigenvalues of the inverse system into the unit circle. Due to the feedback (3.54), the approximate inverse filter becomes

$$
\begin{aligned}
\boldsymbol{x}_{f}[k+1] & =\boldsymbol{\Phi}_{f} \boldsymbol{x}_{f}+\boldsymbol{\Gamma}_{f} \boldsymbol{y}[k+r] \\
\boldsymbol{w}[k] & =\mathbf{C}_{f} \boldsymbol{x}_{f}[k]+\mathbf{D}_{f} \boldsymbol{y}[k+r]
\end{aligned}
$$


where

$$
\begin{aligned}
\mathbf{D}_{f} & =\widetilde{\mathbf{D}}_{f} \\
\mathbf{C}_{f} & =\widetilde{\mathbf{C}}_{f}+\mathbf{F} \\
\boldsymbol{\Gamma}_{f} & =\widetilde{\boldsymbol{\Gamma}}_{f} \\
\boldsymbol{\Phi}_{f} & =\widetilde{\boldsymbol{\Phi}}_{f}+\boldsymbol{\Gamma}_{c} \mathbf{F} .
\end{aligned}
$$

From (3.56d) it becomes evident that $\mathbf{F}$ must be chosen such that the matrix $\widetilde{\boldsymbol{\Phi}}_{f}+\boldsymbol{\Gamma}_{c} \mathbf{F}$ is stable. This can be done by any state-space controller design technique (e.g. any pole placement algorithm).

The filter (3.55) may be used as a feedforward filter to invert system (3.14). Due to the addition of $\boldsymbol{v}[k]$ to the ideal inversion control law in (3.52), the cascade of the filter and the system is not a pure delay of $r$ samples. The precision tracking bandwidth has to be evaluated with the help of the Bode plot of the precision tracking system.

Next, the statement that $\left(\widetilde{\boldsymbol{\Phi}}_{f}, \boldsymbol{\Gamma}_{c}\right)$ is controllable if $\left(\boldsymbol{\Phi}_{c}, \boldsymbol{\Gamma}_{c}\right)$ is controllable shall be shortly demonstrated. Consider the state-transition matrix of the inverse filter in $(3.56 \mathrm{~d})$. With the definiton in $(3.21 \mathrm{c})$, it can be rewritten as

$$
\Phi_{f}=\Phi_{c}+\Gamma_{c}\left(\widetilde{\mathbf{C}}_{f}+\mathbf{F}\right)
$$

Define

$$
\hat{\mathbf{F}}=-\left(\widetilde{\mathbf{C}}_{f}+\mathbf{F}\right)
$$

The eigenvalues of the inverse filter are therefore determined by the eigenvalues of the matrix

$$
\Phi_{f}=\Phi_{c}-\Gamma_{c} \hat{\mathbf{F}}
$$

It becomes clear now that the feedback gain matrix $\hat{\mathbf{F}}$ can place the eigenvalues arbitrarily if the system $\left(\boldsymbol{\Phi}_{c}, \boldsymbol{\Gamma}_{c}\right)$ is controllable. Even if not every eigenvalue of 
$\left(\boldsymbol{\Phi}_{c}, \boldsymbol{\Gamma}_{c}\right)$ is controllable, the feedback $\hat{\mathbf{F}}$ can stabilize the inverse filter if the noncontrollable eigenvalues of $\boldsymbol{\Phi}_{c}$ are stable, which we know is true since we assume that $\boldsymbol{\Phi}_{c}$ is stable. For example, if $\hat{\mathbf{F}}=\mathbf{0}$ is chosen (i.e. $\mathbf{F}=-\widetilde{\mathbf{C}}_{f}$ ), the eigenvalues of $\boldsymbol{\Phi}_{f}$ and $\boldsymbol{\Phi}_{c}$ coincide.

Once the matrix $\hat{\mathbf{F}}$ has been found, it is easy to obtain the feedback gain matrix F:

$$
\mathbf{F}=-\left(\widetilde{\mathbf{C}}_{f}+\hat{\mathbf{F}}\right)
$$

The matrix $\widetilde{\mathbf{C}}_{f}$, which is needed for this computation, only depends on the matrices of system (3.14), i.e. it is known at any point. The rewriting of the inverse filter state-transition matrix $\boldsymbol{\Phi}_{f}$ was only carried out here to demonstrate that $\mathbf{F}$ is able to stabilize $\widetilde{\boldsymbol{\Phi}}_{f}$ over $\boldsymbol{\Gamma}_{c}$; the design of $\mathbf{F}$ in this thesis will always be based on the system $\left(\widetilde{\boldsymbol{\Phi}}_{f}, \boldsymbol{\Gamma}_{c}\right)$ and never on $\left(\boldsymbol{\Phi}_{c}, \boldsymbol{\Gamma}_{c}\right)$.

The idea of adding delays from Section 3.2.2 and the feedback approach presented in this section may also be combined. Assume that $s_{\max }$ denotes the number of advances needed in the algorithm described in Section 3.2.2 to stabilize the inverse filter. Now consider that $s \leq s_{\max }$ advances are added to the $r$ advances shown in (3.15). Again, let $d=r+s$, so (3.38) is obtained and the approximation (3.39) is applied. Next, feedback of the form $\boldsymbol{v}[k]=\mathbf{F} \boldsymbol{x}_{c}[k]$ is added to the resulting control law, so

$\boldsymbol{w}[k]=-\left(\sum_{i=0}^{s} \mathbf{C}_{c} \boldsymbol{\Phi}_{c}^{d-i-1} \boldsymbol{\Gamma}_{c}\right)^{-1} \mathbf{C}_{c} \boldsymbol{\Phi}_{c}^{d} \boldsymbol{x}_{c}[k]+\left(\sum_{i=0}^{s} \mathbf{C}_{c} \boldsymbol{\Phi}_{c}^{d-i-1} \boldsymbol{\Gamma}_{c}\right)^{-1} \boldsymbol{y}[k+d]+\mathbf{F} \boldsymbol{x}_{c}[k]$

follows. The resulting inverse filter is still given by (3.55) and (3.56), only the definitions of $\widetilde{\mathbf{C}}_{f}$ and $\widetilde{\mathbf{D}}_{f}$ in (3.21a) and (3.17) change to

$$
\begin{aligned}
\widetilde{\mathbf{D}}_{f} & =\left(\sum_{i=0}^{s} \mathbf{C}_{c} \boldsymbol{\Phi}_{c}^{d-i-1} \boldsymbol{\Gamma}_{c}\right)^{-1} \\
\widetilde{\mathbf{C}}_{f} & =-\widetilde{\mathbf{D}}_{f} \mathbf{C}_{c} \boldsymbol{\Phi}_{c}^{d}
\end{aligned}
$$


The remaining definitions $\widetilde{\boldsymbol{\Phi}}_{f}=\boldsymbol{\Phi}_{c}+\boldsymbol{\Gamma}_{c} \widetilde{\mathbf{C}}_{f}$ and $\widetilde{\boldsymbol{\Gamma}}_{f}=\boldsymbol{\Gamma}_{c} \widetilde{\mathbf{D}}_{f}$ stay the same, but the new definitions from (3.62) have to be plugged in. Note that the design of $\mathbf{F}$ is slightly different now, since $\widetilde{\boldsymbol{\Phi}}_{f}$ depends on $\widetilde{\mathbf{C}}_{f}$, so if (3.62) is used instead of (3.17), the eigenvalues of $\widetilde{\boldsymbol{\Phi}}_{f}$ will be different. The resulting inverse filter approximately inverts system (3.14) with a delay of $d=r+s \leq d_{\max }$ samples.

The advantage of combining the two methods is that fewer advances than in Section 3.2.2 may be needed, and that the additive term $\boldsymbol{v}[k]$ may be kept "smaller" (and thus does not "disturb" the ideal inversion control law (3.18) as much) if the feedback has to stabilize a filter that inverts the system with $d=r+s$ delays, instead of $r$ delays.

\subsection{Command Shaping Filter}

First, a filter that will be called the command shaping filter (CSF) is presented. It follows the inversion approach shown in Section 3.2. The system (3.14), which will be considered for the inversion, is the feedback tracking system in Fig. 3.1. Let the closed-loop system from $\boldsymbol{w}[k]$ to $\boldsymbol{y}[k]$ be denoted by

$$
\begin{aligned}
\boldsymbol{x}_{c}[k+1] & =\boldsymbol{\Phi}_{c} \boldsymbol{x}_{c}[k]+\boldsymbol{\Gamma}_{c} \boldsymbol{w}[k] \\
\boldsymbol{y}[k] & =\mathbf{C}_{c} \boldsymbol{x}_{c}[k]
\end{aligned}
$$

where

$$
\begin{aligned}
\boldsymbol{x}_{c}[k] & =\left[\begin{array}{c}
\boldsymbol{x}[k] \\
\boldsymbol{x}_{a}[k]
\end{array}\right] & \boldsymbol{\Phi}_{c} & =\left[\begin{array}{cc}
\boldsymbol{\Phi}-\boldsymbol{\Gamma} \mathbf{K}_{1} & \boldsymbol{\Gamma} \mathbf{K}_{2} \\
-\boldsymbol{\Gamma}_{a} \mathbf{C} & \boldsymbol{\Phi}_{a}
\end{array}\right] \\
\boldsymbol{\Gamma}_{c} & =\left[\begin{array}{c}
\mathbf{0} \\
\boldsymbol{\Gamma}_{a}
\end{array}\right] & \mathbf{C}_{c} & =\left[\begin{array}{ll}
\mathbf{C} & 0
\end{array}\right]
\end{aligned}
$$

so for the CSF, the system considered in Section 3.2 is given by (3.63), and the notation in (3.63) coincides with the notation in (3.14).

Next, a short argument shall be given that the system to be inverted contains the zeros of the discrete-time plant model (3.2). In order to do so, it is assumed 
that the order of the additional dynamics is equal to the number of plant outputs, $n_{a}=p$, and that both $\boldsymbol{\Gamma}_{c}$ and $\mathbf{K}_{2}$ are not rank deficient, i.e. they are $p \times p$ matrices with a nonzero determinant. The invariant zeros of system (3.63) are found via its Rosenbrock matrix $\mathbf{P}_{c}(z)$ (see also Section 3.2.1):

$$
\operatorname{det}\left(\mathbf{P}_{c}(z)\right)=\operatorname{det}\left(\left[\begin{array}{ccc}
z \mathbf{I}-\mathbf{\Phi}+-\boldsymbol{\Gamma} \mathbf{K}_{1} & \boldsymbol{\Gamma}_{a} \mathbf{K}_{2} & \mathbf{0} \\
\boldsymbol{\Gamma}_{a} \mathbf{C} & z \mathbf{I}-\mathbf{\Phi}_{a} & -\boldsymbol{\Gamma} \\
\mathbf{C} & \mathbf{0} & \mathbf{0}
\end{array}\right]\right)=0
$$

It is known that

$$
\operatorname{det}(\mathbf{A B})=\operatorname{det}(\mathbf{A}) \cdot \operatorname{det}(\mathbf{B})
$$

holds for $\mathbf{A}, \mathbf{B} \in \mathbb{R}^{n}$. Define a matrix

$$
\mathbf{E}=\left[\begin{array}{ccc}
\mathbf{I}_{n} & \mathbf{0} & \mathbf{0} \\
\mathbf{0} & \mathbf{0} & \mathbf{I}_{p} \\
\mathbf{0} & \mathbf{I}_{p} & \mathbf{0}
\end{array}\right]
$$

Since $|\operatorname{det}(\mathbf{E})|=1 \neq 0,(3.65)$ can be expressed as

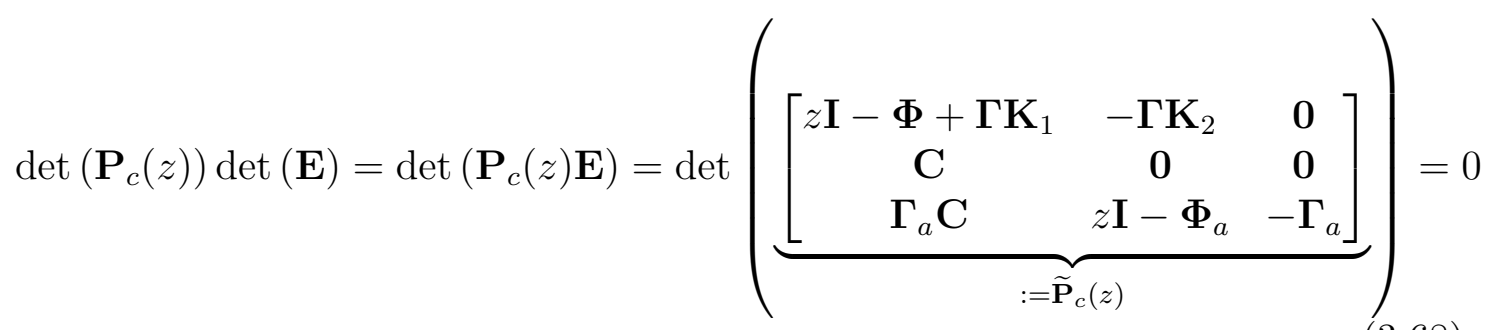

so that we can as well find the values $\eta_{i}$ for which $\widetilde{\mathbf{P}}_{c}\left(\eta_{i}\right)$ is rank deficient in order to find the invariant zeros of (3.63). Next, the rule

$$
\operatorname{det}\left(\left[\begin{array}{ll}
\mathbf{A} & \mathbf{B} \\
\mathbf{C} & \mathbf{D}
\end{array}\right]\right)=\operatorname{det}\left(\mathbf{A}-\mathbf{B D}^{-1} \mathbf{C}\right) \cdot \operatorname{det}(\mathbf{D})
$$

is used [36], and since $\mathbf{B}=\mathbf{0}$ in our case and it is assumed that $\boldsymbol{\Gamma}_{a}$ is a square matrix with full rank

$$
\operatorname{det}(\widetilde{\mathbf{P}}(z))=\operatorname{det}\left(\left[\begin{array}{cc}
z \mathbf{I}-\mathbf{\Phi}+\boldsymbol{\Gamma} \mathbf{K}_{1} & -\boldsymbol{\Gamma} \mathbf{K}_{2} \\
\mathbf{C} & \mathbf{0}
\end{array}\right]\right) \cdot \operatorname{det}\left(-\boldsymbol{\Gamma}_{a}\right)=0
$$

follows. This equation can be further rewritten

$$
(-1)^{p} \cdot \operatorname{det}\left(\boldsymbol{\Gamma}_{a}\right) \cdot \operatorname{det}\left(\left[\begin{array}{cc}
z \mathbf{I}-\mathbf{\Phi} & -\boldsymbol{\Gamma} \\
\mathbf{C} & \mathbf{0}
\end{array}\right]\left[\begin{array}{cc}
\mathbf{I} & \mathbf{0} \\
-\mathbf{K}_{1} & \mathbf{K}_{2}
\end{array}\right]\right)=0
$$


and due to our assumption that $\mathbf{K}_{2}$ and $\boldsymbol{\Gamma}_{a}$ have full rank, we finally obtain

$$
\operatorname{det}\left(\boldsymbol{\Gamma}_{a}\right) \cdot \operatorname{det}\left(\mathbf{K}_{2}\right) \cdot \operatorname{det}(\underbrace{\left[\begin{array}{cc}
z \mathbf{I}-\mathbf{\Phi} & -\boldsymbol{\Gamma} \\
\mathbf{C} & \mathbf{0}
\end{array}\right]}_{=\mathbf{P}(z)})=0 \quad \Rightarrow \operatorname{det}(\mathbf{P}(z))=0
$$

with $\operatorname{det}\left(\boldsymbol{\Gamma}_{a}\right) \neq 0$ and $\operatorname{det}\left(\mathbf{K}_{2}\right) \neq 0$. The matrix $\mathbf{P}(z)$, however, is the Rosenbrock matrix of the discrete-time plant (3.2). Thus the invariant zeros of (3.63) and (3.2) coincide if the order of the additional dynamics match the number of plant outputs and both $\boldsymbol{\Gamma}_{a}$ and $\mathbf{K}_{2}$ have full rank $\left(\boldsymbol{\Gamma}_{a}\right.$ has full rank if integral additional dynamics are used). If these conditions are not met, the argumentation has to be modified.

Since the CSF is the inverse of a closed-loop system, it belongs to the class of closed-loop-inversion feedforward (CLIF) architectures [11, 12], and we know that the closed-loop system (3.63) is stable. The CSF $\left(\boldsymbol{\Phi}_{f}, \boldsymbol{\Gamma}_{f}, \mathbf{C}_{f}, \mathbf{D}_{f}\right)$ can be derived by using either of the algorithms shown in Section 3.2. If the feedback approach from Section 3.2.3 is applied, it should be checked whether the system (3.63) is controllable, but it is assumed that this should be the case if no pole/zero cancellations occur during the design process of the feedback tracking system. If they do occur and the system is not controllable, a model reduction and inverting the reduced system may solve the problem. It is noted that, according to Section 3.2 , the feedback is always able to stabilize the inverse filter; however, it may not be possible to place its eigenvalues arbitrarily.

An advantage of the CSF is that the feedback design in Section 3.1 determines the eigenvalues of the state-transition matrix $\boldsymbol{\Phi}_{c}$, which is inverted in Section 3.2. Thus, it is ensured that $\boldsymbol{\Phi}_{c}$ is stable. Additionally, if all eigenvalues of $\boldsymbol{\Phi}_{c}$ are distinct (which can be achieved by design), the matrix $\mathbf{T}$ which diagonalizes $\boldsymbol{\Phi}_{c}$ is known to have full rank [37], so its condition number in (3.45) will be finite.

A precision tracking system is obtained by inserting a feedforward CSF into 


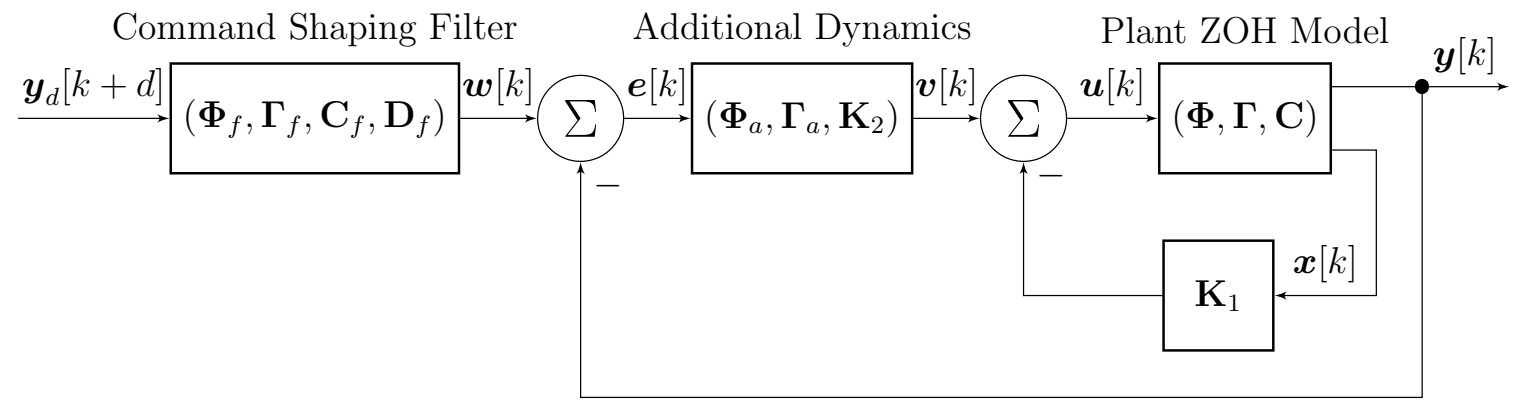

Figure 3.5: Precision Tracking System - Command Shaping Filter

Fig. 3.1 and accounting for the delay of $d$ samples, as shown in Fig. 3.5. Here, $\boldsymbol{y}_{d}[k]$ denotes the desired output trajectory.

\subsection{Inverse Modified Plant}

The second inverse filter that is considered in this thesis is the so-called inverse modified plant (IMP). The difference between the IMP and the CSF presented in Section 3.3 is that not the model of the entire feedback tracking system in Fig. 3.1 is inverted, but the model for the system from $\boldsymbol{v}[k]$ to $\boldsymbol{y}[k]$, which will be called the modified plant. It is given as follows:

$$
\begin{aligned}
\boldsymbol{x}[k+1] & =\left(\boldsymbol{\Phi}-\boldsymbol{\Gamma} \mathbf{K}_{1}\right) \boldsymbol{x}[k]+\boldsymbol{\Gamma} \boldsymbol{v}[k] \\
\boldsymbol{y}[k] & =\mathbf{C} \boldsymbol{x}[k] .
\end{aligned}
$$

Thus, for the IMP, the system (3.14), which is inverted in Section 3.2, corresponds to (3.73). The system (3.73) has the same invariant zeros as the discrete-time plant (3.2). This can be shown with its Rosenbrock matrix:

$$
\begin{aligned}
\operatorname{det}\left(\mathbf{P}_{c}(z)\right) & =\operatorname{det}\left(\left[\begin{array}{cc}
z \mathbf{I}-\mathbf{\Phi}+\boldsymbol{\Gamma} \mathbf{K}_{1} & -\boldsymbol{\Gamma} \\
\mathbf{C} & \mathbf{0}
\end{array}\right]\right)=\operatorname{det}\left(\left[\begin{array}{cc}
z \mathbf{I}-\mathbf{\Phi} & -\boldsymbol{\Gamma} \\
\mathbf{C} & \mathbf{0}
\end{array}\right]\left[\begin{array}{cc}
\mathbf{I} & \mathbf{0} \\
-\mathbf{K}_{1} & \mathbf{I}
\end{array}\right]\right) \\
& =\operatorname{det}(\underbrace{\left[\begin{array}{cc}
z \mathbf{I}-\mathbf{\Phi} & -\boldsymbol{\Gamma} \\
\mathbf{C} & \mathbf{0}
\end{array}\right]}_{=\mathbf{P}(z)}) \cdot \underbrace{\operatorname{det}\left(\left[\begin{array}{cc}
\mathbf{I} & \mathbf{0} \\
-\mathbf{K}_{1} & \mathbf{I}
\end{array}\right]\right)}_{=1}=\operatorname{det}(\mathbf{P}(z))=0
\end{aligned}
$$

where $\mathbf{P}(z)$ denotes the Rosenbrock matrix of the plant (3.2). 


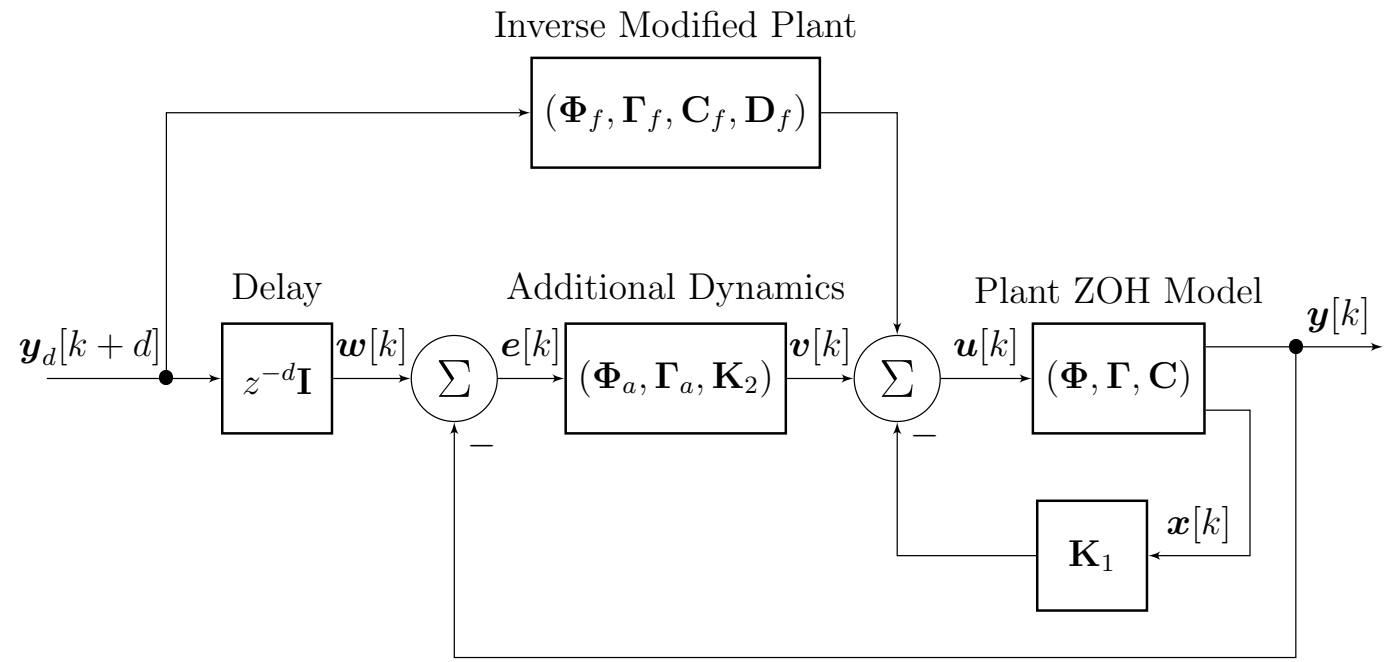

Figure 3.6: Precision Tracking System - Inverse Modified Plant

After the IMP $\left(\boldsymbol{\Phi}_{f}, \boldsymbol{\Gamma}_{f}, \mathbf{C}_{f}, \mathbf{D}_{f}\right)$ has been found, using one of the algorithms presented in Section 3.2, it has to be inserted into the feedback tracking system in Fig. 3.1 to obtain a precision tracking system. Furthermore, the delay of $d$ samples has to be accounted for. The result can be seen in Fig. 3.6. Again, the desired output trajectory is denoted by $\boldsymbol{y}_{d}[k]$.

The IMP belongs to the class of plant inversion feedforward (PIF) architectures (see Fig. 2.1b). Even though the modified plant in Fig. 3.6 consists of a feedback loop (closed by $\mathbf{K}_{1}$ ), it is not guaranteed by the feedback design discussed in Section 3.1 that $\mathbf{\Phi}_{c}=\mathbf{\Phi}-\boldsymbol{\Gamma} \mathbf{K}_{1}$ is stable (it is only guaranteed that $\boldsymbol{\Phi}_{d}-\boldsymbol{\Gamma}_{d} \mathbf{K}_{d}$ possesses the desired pole locations, so the feedback design is not based on the system $(\boldsymbol{\Phi}, \boldsymbol{\Gamma}))$. For that reason, the IMP is classified as PIF. However, since the algorithm in Section 3.2 assumes that the system to be inverted is stable, it should be checked whether the eigenvalues of $\boldsymbol{\Phi}_{c}=\boldsymbol{\Phi}-\boldsymbol{\Gamma} \mathbf{K}_{1}$ are all located inside the unit circle or not. If this is not the case, the feedback matrix $\mathbf{K}_{d}$ may be recalculated (e.g. by selecting new pole locations or using a different design technique), or the usage of the CSF from Section 3.3 may be considered. 


\subsection{Inclusion of a State Observer}

The preceding precision tracking architectures assumed that all the state variables of the plant are available in the design of the feedback tracking system. In practice, however, this will not always be the case. Therefore, this section focuses on the question whether and how the inclusion of a state observer will affect the CSF and IMP precision tracking systems in Sections 3.3 and 3.4, respectively.

Consider system (3.2). A state observer provides an estimate $\hat{\boldsymbol{x}}[k]$ for the actual state vector $\boldsymbol{x}[k][26]$ :

$$
\begin{aligned}
\hat{\boldsymbol{x}}[k+1] & =\boldsymbol{\Phi} \hat{\boldsymbol{x}}[k]+\boldsymbol{\Gamma} \boldsymbol{u}[k]+\mathbf{L}(\boldsymbol{y}[k]-\hat{\boldsymbol{y}}[k]) \\
\hat{\boldsymbol{y}}[k] & =\mathbf{C} \hat{\boldsymbol{x}}[k]
\end{aligned}
$$

Here, $\mathbf{L}$ denotes the observer gain. Let $\boldsymbol{e}_{x}[k]=\boldsymbol{x}[k]-\hat{\boldsymbol{x}}[k]$ be the observer error, SO

$$
\boldsymbol{e}_{x}[k+1]=(\boldsymbol{\Phi}-\mathbf{L C}) \boldsymbol{e}_{x}[k]
$$

follows. A feedback tracking system which includes a plant, additional dynamics and state observer, can therefore be written as

$$
\begin{aligned}
{\left[\begin{array}{c}
\boldsymbol{x}[k+1] \\
\boldsymbol{x}_{a}[k+1] \\
\boldsymbol{e}_{x}[k+1]
\end{array}\right] } & =\left[\begin{array}{ccc}
\boldsymbol{\Phi}-\boldsymbol{\Gamma K}_{1} & \boldsymbol{\Gamma} \mathbf{K}_{2} & \mathbf{0} \\
-\boldsymbol{\Gamma}_{a} & \boldsymbol{\Phi}_{a} & \mathbf{0} \\
\mathbf{0} & \mathbf{0} & \boldsymbol{\Phi}-\mathbf{L C}
\end{array}\right]\left[\begin{array}{c}
\boldsymbol{x}[k] \\
\boldsymbol{x}_{a}[k] \\
\boldsymbol{e}_{x}[k]
\end{array}\right]+\left[\begin{array}{c}
\mathbf{0} \\
\boldsymbol{\Gamma}_{a} \\
\mathbf{0}
\end{array}\right] \mathbf{w}[k] \\
\boldsymbol{y}[k] & =\left[\begin{array}{lll}
\mathbf{C} & \mathbf{0} & \mathbf{0}
\end{array}\right]\left[\begin{array}{c}
\boldsymbol{x}[k] \\
\boldsymbol{x}_{a}[k] \\
\boldsymbol{e}_{x}[k]
\end{array}\right]
\end{aligned}
$$

Since the state-transition matrix of the feedback tracking system in (3.77) is a block diagonal matrix, the eigenvalues of the tracking system are determined by the eigenvalues of the matrices $\left[\begin{array}{cc}\boldsymbol{\Phi}-\boldsymbol{\Gamma} \mathbf{K}_{1} & \boldsymbol{\Gamma} \mathbf{K}_{2} \\ -\boldsymbol{\Gamma}_{a} & \boldsymbol{\Phi}_{a}\end{array}\right]$ and $[\boldsymbol{\Phi}-\mathbf{L C}]$. The first matrix can be rewritten as $\boldsymbol{\Phi}_{d}-\boldsymbol{\Gamma}_{d} \mathbf{K}_{d}$, with the matrices $\left(\boldsymbol{\Phi}_{d}, \boldsymbol{\Gamma}_{d}, \mathbf{K}_{d}\right)$ being defined in (3.8) in Section 3.1, and the latter matrix describes the dynamics of the observer

error. Hence, the feedback gain $\mathbf{K}_{d}$ and the observer gain $\mathbf{L}$ can be designed independently, i.e. the observer gain does not affect the eigenvalues of the feedback 
tracking system shown in Fig. 3.1. A recommendation for the eigenvalue locations of the observer dynamics can be found in [27].

When an observer is used in the feedback tracking system in Fig. 3.1, the system $\left(\boldsymbol{\Phi}_{c}, \boldsymbol{\Gamma}_{c}, \mathbf{C}_{c}\right)$ that is inverted in Section 3.2 has to be adjusted for both CSF and IMP. For the CSF, the system from $\boldsymbol{w}[k]$ to $\boldsymbol{y}[k]$ is inverted, which is given by

$$
\begin{aligned}
{\left[\begin{array}{c}
\boldsymbol{x}[k+1] \\
\boldsymbol{x}_{a}[k+1] \\
\hat{\boldsymbol{x}}[k+1]
\end{array}\right] } & =\underbrace{\left[\begin{array}{ccc}
\boldsymbol{\Phi}-\boldsymbol{\Gamma K}_{1} & \boldsymbol{\Gamma \mathbf { K } _ { 2 }} & \mathbf{0} \\
-\boldsymbol{\Gamma}_{a} & \boldsymbol{\Phi}_{a} & \mathbf{0} \\
\mathbf{L C} & \boldsymbol{\Gamma \boldsymbol { K } _ { 2 }} & \boldsymbol{\Phi}-\mathbf{L C}
\end{array}\right]}_{=\boldsymbol{\Phi}_{c}}\left[\begin{array}{c}
\boldsymbol{x}[k] \\
\boldsymbol{x}_{a}[k] \\
\hat{\boldsymbol{x}}[k]
\end{array}\right]+\underbrace{\left[\begin{array}{c}
\mathbf{0} \\
\boldsymbol{\Gamma}_{a} \\
\mathbf{0}
\end{array}\right]}_{=\boldsymbol{\Gamma}_{c}} \mathbf{w}[k] \\
\boldsymbol{y}[k] & =\underbrace{\left[\begin{array}{lll}
\mathbf{C} & \mathbf{0} & \mathbf{0}
\end{array}\right]}_{=\mathbf{C}_{c}}\left[\begin{array}{c}
\boldsymbol{x}[k] \\
\boldsymbol{x}_{a}[k] \\
\hat{\boldsymbol{x}}[k]
\end{array}\right] .
\end{aligned}
$$

The IMP, in contrast, inverts the system from $\boldsymbol{v}[k]$ to $\boldsymbol{y}[k]$ :

$$
\begin{aligned}
{\left[\begin{array}{c}
\boldsymbol{x}[k+1] \\
\hat{\boldsymbol{x}}[k+1]
\end{array}\right] } & =\underbrace{\left[\begin{array}{cc}
\boldsymbol{\Phi} & -\boldsymbol{\Gamma} \mathbf{K}_{1} \\
\mathbf{L C} & \boldsymbol{\Phi}-\mathbf{L C}-\boldsymbol{\Gamma} \mathbf{K}_{1}
\end{array}\right]}_{=\boldsymbol{\Phi}_{c}}\left[\begin{array}{l}
\boldsymbol{x}[k] \\
\hat{\boldsymbol{x}}[k]
\end{array}\right]+\underbrace{\left[\begin{array}{c}
\boldsymbol{\Gamma} \\
\boldsymbol{\Gamma}
\end{array}\right]}_{=\boldsymbol{\Gamma}_{c}} \boldsymbol{v}[k] \\
\boldsymbol{y}[k] & =\underbrace{\left[\begin{array}{ll}
\mathbf{C} & \mathbf{0}
\end{array}\right]}_{=\mathbf{C}_{c}}\left[\begin{array}{l}
\boldsymbol{x}[k] \\
\hat{\boldsymbol{x}}[k]
\end{array}\right] .
\end{aligned}
$$

Furthermore, the systems (3.11) and (3.13), which are used to calculate the robustness bounds $\delta_{1}$ and $\delta_{2}$ in Section 3.1, have to be adjusted if an observer is added to the feedback tracking system. System $\mathbf{H}_{1}(z)$ is now given by

$$
\begin{aligned}
{\left[\begin{array}{c}
\boldsymbol{x}[k+1] \\
\boldsymbol{x}_{a}[k+1] \\
\hat{\boldsymbol{x}}[k+1]
\end{array}\right] } & =\left[\begin{array}{ccc}
\boldsymbol{\Phi}-\boldsymbol{\Gamma} \mathbf{K}_{1} & \boldsymbol{\Gamma} \mathbf{K}_{2} & -\boldsymbol{\Gamma} \mathbf{K}_{1} \\
-\boldsymbol{\Gamma}_{a} \mathbf{C} & \boldsymbol{\Phi}_{a} & \mathbf{0} \\
\mathbf{L C} & \boldsymbol{\Gamma} \mathbf{K}_{2} & \boldsymbol{\Phi}-\mathbf{L C}-\boldsymbol{\Gamma} \mathbf{K}_{1}
\end{array}\right]\left[\begin{array}{c}
\boldsymbol{x}[k] \\
\boldsymbol{x}_{a}[k] \\
\hat{\boldsymbol{x}}[k]
\end{array}\right]+\left[\begin{array}{c}
\boldsymbol{\Gamma} \\
\mathbf{0} \\
\mathbf{0}
\end{array}\right] \boldsymbol{w}[k] \\
\boldsymbol{v}[k] & =\left[\begin{array}{lll}
\mathbf{0} & \mathbf{K}_{2} & -\mathbf{K}_{1}
\end{array}\right]\left[\begin{array}{c}
\boldsymbol{x}[k] \\
\boldsymbol{x}_{a}[k] \\
\hat{\boldsymbol{x}}[k]
\end{array}\right]
\end{aligned}
$$

whereas

$$
\begin{aligned}
{\left[\begin{array}{c}
\boldsymbol{x}[k+1] \\
\boldsymbol{x}_{a}[k+1] \\
\hat{\boldsymbol{x}}[k+1]
\end{array}\right] } & =\left[\begin{array}{ccc}
\boldsymbol{\Phi}-\boldsymbol{\Gamma} \mathbf{K}_{1} & \boldsymbol{\Gamma} \mathbf{K}_{2} & -\boldsymbol{\Gamma} \mathbf{K}_{1} \\
-\boldsymbol{\Gamma}_{a} \mathbf{C} & \boldsymbol{\Phi}_{a} & \mathbf{0} \\
\mathbf{L C} & \boldsymbol{\Gamma} \mathbf{K}_{2} & \boldsymbol{\Phi}-\mathbf{L C}-\boldsymbol{\Gamma} \mathbf{K}_{1}
\end{array}\right]\left[\begin{array}{c}
\boldsymbol{x}[k] \\
\boldsymbol{x}_{a}[k] \\
\hat{\boldsymbol{x}}[k]
\end{array}\right]+\left[\begin{array}{c}
\boldsymbol{\Gamma} \\
\mathbf{0} \\
\mathbf{0}
\end{array}\right] \boldsymbol{w}[k] \\
\boldsymbol{v}[k] & =\left[\begin{array}{lll}
\mathbf{0} & \mathbf{K}_{2} & -\mathbf{K}_{1}
\end{array}\right]\left[\begin{array}{c}
\boldsymbol{x}[k] \\
\boldsymbol{x}_{a}[k] \\
\hat{\boldsymbol{x}}[k]
\end{array}\right]+\mathbf{I}_{p} \boldsymbol{w}[k]
\end{aligned}
$$


holds for $\mathbf{H}_{2}(z)$. Please note that $\boldsymbol{w}[k]$ in (3.80) and (3.81) refers to the notation in Fig. 3.2 and Fig. 3.3, not to the notation in Fig 3.1. 


\section{CHAPTER 4}

\section{Feedback Inversion Design Techniques}

This chapter deals with possibilities to design the feedback matrix $\mathbf{F}$, which was introduced in Section 3.2.3 to stabilize the inverse filter. Clearly, $\mathbf{F}$ has to move

the eigenvalues of $\boldsymbol{\Phi}_{f}=\widetilde{\boldsymbol{\Phi}}_{f}+\boldsymbol{\Gamma}_{c} \mathbf{F}$ inside the unit circle, but there are multiple ways how this can be done. Since there are no strict rules for the exact pole locations of the inverse filter, this degree of freedom can be used to try out different design approaches.

A first idea is to use "classical" feedback design techniques, such as poleplacement or dlqr. For pole-placement, the inverse filter's pole location could be based on the influence of pole and zero locations of a dynamic system on its frequency response, and the weights for a dlqr design on the demand that the control effort deriving from the addition of the feedback is low. Thoughts on these design techniques are presented in Section 4.1.

As another step, a design was developed in this thesis which focuses on finding a matrix $\mathbf{F}$ which stabilizes the system while having a minimal norm. It is assumed that the influence of the feedback on the ideal inversion control law is reduced if the norm of $\mathbf{F}$ is minimal. This design is explicated in Section 4.2.

Finally, inspired by the optimization in Section 4.2, another optimization design is introduced in Section 4.3. The main idea is to design $\mathbf{F}$ so that it "compensates" the deviation of the tracking system's frequency response from the ideal frequency response (due to the approximation in (3.39)), while a stable inverse filter is guaranteed. From all design techniques that were considered in order to calculate $\mathbf{F}$, this proved to be the most successful, which is why it will be considered as the main design method for the inversion via feedback in the discussion in 
Chapter 5 .

\subsection{Classical Feedback Design}

The easiest way to design the feedback matrix $\mathbf{F}$ is to choose the desired locations for the filter with the feedback included. For the discussion of the "closedloop" poles, we assume that the state-transition matrix $\boldsymbol{\Phi}_{f}$ is given as

$$
\boldsymbol{\Phi}_{f}=\widetilde{\boldsymbol{\Phi}}_{f}+\boldsymbol{\Gamma}_{c} \mathbf{F}
$$

where $\widetilde{\boldsymbol{\Phi}}_{f}$ is defined according to $(3.21 \mathrm{c})$, i.e. $\widetilde{\boldsymbol{\Phi}}_{f}$ is the state-transition matrix of the (unstable) filter which inverts system (3.14) with a pure delay of $r$ samples (see Section 3.2.3 for details). Consequently, the eigenvalues of $\widetilde{\boldsymbol{\Phi}}_{f}$ are moved with the help of $\mathbf{F}$ to obtain the eigenvalues of $\boldsymbol{\Phi}_{f}$. Assume that system (3.14) has $n_{z}$ zeros. Then, $n_{z}$ eigenvalues of $\widetilde{\boldsymbol{\Phi}}_{f}$ coincide with the zeros of (3.14). The remaining $n_{c}-n_{z}$ eigenvalues are located in zero. Since the idea of the feedback approach is to simply stabilize the filter, without interfering too much with the ideal inversion control law (3.18), it is proposed here to keep all the stable eigenvalues in their respective spot and just move the unstable eigenvalues to obtain a stable filter. Sometimes, problems might arise if the attempt is made to place multiple eigenvalues in the same spot (e.g. the $n_{c}-n_{z}$ eigenvalues in zero). In this thesis, the eigenvalues were not placed exactly in zero, but $n_{c}-n_{z}$ equally spaced eigenvalues at radius $R_{\text {zero }}$ were placed around the origin.

There are several possibilities how to choose the "closed-loop" locations of the unstable eigenvalues of $\widetilde{\boldsymbol{\Phi}}_{f}$. The simplest way to determine the desired eigenvalue locations is to choose a spot in the stability region that is very close to the original position of the eigenvalue (e.g. an eigenvalue in 1.1 could be moved to 0.99 and an eigenvalue in -1.1 to -0.99$)$.

Another possibility is to reflect the unstable eigenvalues into the unit circle, so that the magnitude is maintained in the Bode plot. Assume that $\widetilde{\boldsymbol{\Phi}}_{f}$ has $\widetilde{n}_{z}$ 
unstable eigenvalues, that are given as the roots of the polynomial

$$
B_{u}=b_{\widetilde{n}_{z}} z^{\widetilde{n}_{z}}+b_{\widetilde{n}_{z}-1} z^{\widetilde{n}_{z}-1}+\cdots+b_{1} z+b_{0}
$$

Then, the roots of the polynomial

$$
B_{s}=b_{0} z^{\widetilde{n}_{z}}+b_{1} z^{\widetilde{n}_{z}-1}+\cdots+b_{\widetilde{n}_{z}-1} z+b_{\widetilde{n}_{z}}
$$

should be chosen as the eigenvalues of $\boldsymbol{\Phi}_{f}[13]$.

If the system under consideration is a MIMO system, it is proposed to select the eigenvalues according to this section, and to simultaneously try to minimize the influence of $\mathbf{F}$ (i.e. $\boldsymbol{v}[k]$ ) in the inversion control law (3.52). A reasonable approach is to minimize a norm $\|\mathbf{F}\|$, which was achieved in this thesis by adapating the algorithm described in [27].

Another possibility to calculate $\mathbf{F}$ is via a $d l q r$ design. For a state-space system with $n$ variables and $p$ inputs, a $d l q r$ design minimizes the following quadratic function of the states and inputs:

$$
J_{\mathrm{dlqr}}=\frac{1}{2} \sum_{k=0}^{\infty}\left(\boldsymbol{x}^{\mathrm{T}}[k] \mathbf{Q} \boldsymbol{x}[k]+\boldsymbol{u}^{\mathrm{T}}[k] \mathbf{S} \boldsymbol{u}[k]\right)
$$

where $\mathbf{Q} \in \mathbb{R}^{n \times n}$ and $\mathbf{S} \in \mathbb{R}^{p \times p}$ are symmetric, positive-definite weighting matrices. If $\mathbf{S}$ is large with respect to $\mathbf{Q}$, the resulting regulator will stabilize the plant without using much control effort [26]. In the light of this discussion, a dlqr design for $\mathbf{F}$ may be used with $\mathbf{S}$ being much larger than $\mathbf{Q}$. As a reminder: the term $\boldsymbol{v}[k]=\mathbf{F} \boldsymbol{x}_{c}[k]$ was added to the inversion control law (3.18) to stabilize the inverse filter, so it can be argued that the tracking performance will be best if the control effort $\boldsymbol{v}[k]$ is as small as possible, while it is still large enough to stabilize the filter.

\subsection{Norm Minimization Approach}

One of the feedback design approaches that is considered and developed in this thesis is to stabilize the inverse filter and simultaneously attempt to minimize 
the Frobenius norm of the resulting gain matrix $\mathbf{F}$. The idea behind this is that if the norm of $\mathbf{F}$ is minimal, the influence of the additive term $\boldsymbol{v}[k]=\mathbf{F} \boldsymbol{x}_{c}[k]$ (see Section 3.2.3) will probably also be limited.

In order to design the matrix $\mathbf{F}$, an idea from [9] is used. The author designs feedback to stabilize the inverse of a continuous-time hypersonic vehicle plant, which has one non-minimum phase zero. The basic idea is generalized here to the general case of multiple (and possibly complex) non-minimum phase zeros for a digital system.

The result of the design process is a set of linear equality constraints and nonlinear inequality constraints, which could be solved "by hand" and, by themselves, do not ensure a minimum norm. An advantage of this design is that an analytical relation between the eigenvalues of the stabilized inverse filter and the entries of $\mathbf{F}$ is obtained. In this thesis, they were included in an optimization problem which minimizes the norm of the feedback gain matrix. One of the (theoretic) advantages of minimizing the norm is the Lagrange dualism could be applied to the problem, and the Lagrange dual problem is known to be convex [38].

This section is organized as follows: first, the constraints for the entries of the gain matrix $\mathbf{F}$ are derived. After this, they are included in an optimization problem, which is solved using the Lagrange dualism. A discussion of the approach is given in the last subsection.

\subsubsection{Constraints for the Feedback Gain Matrix}

Following the idea in [9], it is desired that the matrix $\mathbf{F}$ moves the unstable eigenvalues of the inverse filter (resulting from the plant's non-minimum phase zeros) into the stability region, while it keeps the remaining (stable) eigenvalues in their respective spot. In order to do so, system (3.53) is transformed to canonical 
modal form. A state transformation

$$
\boldsymbol{x}_{c}[k]=\mathbf{V} \boldsymbol{\psi}[k]
$$

is introduced, so

$$
\boldsymbol{\psi}[k+1]=\mathbf{V}^{-1} \widetilde{\boldsymbol{\Phi}}_{f} \mathbf{V} \boldsymbol{\psi}[k]+\mathbf{V}^{-1} \widetilde{\boldsymbol{\Gamma}}_{f} \boldsymbol{y}_{d}[k]+\mathbf{V}^{-1} \boldsymbol{\Gamma}_{c} \underbrace{\mathbf{F} \mathbf{V} \boldsymbol{x}_{c}[k]}_{=\boldsymbol{v}[k]}
$$

is the result. The transformation matrix $\mathbf{V}$ is the right eigenvector matrix of $\widetilde{\boldsymbol{\Phi}}_{f}$, but if $\lambda_{j, j+1}$ is a conjugate-complex eigenvalue pair of $\widetilde{\boldsymbol{\Phi}}_{f}$, the $j$ th column is replace by the real part of the $j$ th eigenvector, and the $(j+1)$ th column by the imaginary part of the $j$ th eigenvalue. One realization aspect that was not discussed in [9], however, is that $\widetilde{\boldsymbol{\Phi}}_{f}$ possesses a multiple eigenvalue in 0 (with multiplicity $p \cdot r$, see Section 3.2.1), and usually there are less than $p \cdot r$ linearly independent eigenvectors associated with the eigenvalue in 0 (i.e. $\mathbf{V}$ would be rank-deficient). For that reason, generalzed eigenvectors have to be used [37]. Let $\boldsymbol{v}_{i}^{(1)}$ be an eigenvector to the eigenvalue 0 . Then the generalized eigenvector of second order, denoted by $\boldsymbol{v}_{i}^{(2)}$ can be calculated according to [37]:

$$
\widetilde{\boldsymbol{\Phi}}_{f} \boldsymbol{v}_{i}^{(2)}=\boldsymbol{v}_{i}^{(1)}
$$

The usage of the generalized eigenvectors results in Jordan blocks for the multiple eigenvalue in 0 , i.e. in ones above the main diagonal.

With the definitons

$$
\begin{aligned}
\mathbf{W} & :=\mathbf{V}^{-1} \\
\boldsymbol{\Lambda} & :=\mathbf{W} \widetilde{\boldsymbol{\Phi}}_{f} \mathbf{V} \\
\boldsymbol{\Gamma}_{\psi} & :=\mathbf{W} \boldsymbol{\Gamma}_{c} \\
\boldsymbol{\Gamma}_{y} & :=\mathbf{W} \widetilde{\boldsymbol{\Gamma}}_{f} \\
\hat{\mathbf{F}} & :=-\mathbf{F V}
\end{aligned}
$$


we get

$$
\boldsymbol{\psi}[k+1]=\boldsymbol{\Lambda} \boldsymbol{\psi}[k]-\boldsymbol{\Gamma}_{\psi} \hat{\mathbf{F}} \boldsymbol{\psi}[k]+\boldsymbol{\Gamma}_{y} \boldsymbol{y}[k+r]
$$

where $\boldsymbol{\Lambda}$ is in canonical modal form. Next, the partitionings

$$
\begin{aligned}
& \hat{\mathbf{F}}=\left[\begin{array}{lll}
\hat{\boldsymbol{f}}_{1} & \ldots & \hat{\boldsymbol{f}}_{n_{c}}
\end{array}\right] \\
& \boldsymbol{\Gamma}_{\psi}=\left[\begin{array}{c}
\gamma_{\psi, 1} \\
\vdots \\
\gamma_{\psi, n_{c}}
\end{array}\right] \\
& \boldsymbol{\Gamma}_{y}=\left[\begin{array}{c}
\gamma_{y, 1} \\
\vdots \\
\gamma_{y, n_{c}}
\end{array}\right]
\end{aligned}
$$

are introduced. Furthermore, it is assumed (in this section) that the first $n_{z}$ eigenvalues of $\boldsymbol{\Lambda}$ are located outside the unit circle (without loss of generality). These are the eigenvalues that correspond to the non-minimum phase zeros of (3.14). It is also assumed that the first $2 n_{i}$ eigenvalues within the first $n_{z}$ unstable eigenvalues are complex (i.e. there are $n_{i}$ pairs of of conjugate-complex unstable eigenvalues). The conjugate complex eigenvalues of $\boldsymbol{\Lambda}$ are denoted by $\delta_{j} \pm \omega_{j}$ and the real eigenvalues by $\lambda_{j}$. With these definitons and assumptions, we have

$$
\left.\begin{array}{c}
\psi_{j}[k+1]=\delta_{j} \psi_{j}[k]+\omega_{j} \psi_{j+1}[k]-\sum_{l=1}^{n_{c}} \gamma_{\psi, j}^{\mathrm{T}} \hat{\boldsymbol{f}}_{l} \psi_{l}[k]+\boldsymbol{\psi}_{y, j} \boldsymbol{y}[k+r] \\
\psi_{j+1}[k+1]=-\omega_{j} \psi_{j}[k]+\delta_{j} \psi_{j+1}[k]-\sum_{l=1}^{n_{c}} \boldsymbol{\gamma}_{\psi, j+1}^{\mathrm{T}} \hat{\boldsymbol{f}}_{l} \psi_{l}[k]+\boldsymbol{\psi}_{y, j+1} \boldsymbol{y}[k+r]
\end{array}\right\} j=1,3, \ldots, 2 n_{i}-1
$$

for the first $2 n_{i}$ modal states (which are affected by the complex unstable eigenvalues of $\widetilde{\boldsymbol{\Phi}}_{f}$ ) and

$\psi_{j}[k+1]=\lambda_{j} \psi_{j}[k]-\sum_{l=1}^{n_{c}} \boldsymbol{\gamma}_{\psi, j+1}^{\mathrm{T}} \hat{\boldsymbol{f}}_{l} \psi_{l}[k]+\boldsymbol{\gamma}_{y, j} \boldsymbol{y}[k+r] \quad j=2 n_{i}+1,2 n i+2, \ldots, n_{z}$

for the next $n_{z}-2 n_{i}$ modal states (which are affected by the real-valued unstable eigenvalues of $\left.\widetilde{\boldsymbol{\Phi}}_{f}\right)$. The remaining $n_{c}-n_{z}$ modal states are only affected by the 
stable eigenvalues of $\widetilde{\boldsymbol{\Phi}}_{f}$, which we do not wish to move.

Following the idea in [9], we want the modal states associated with the unstable eigenvalues (i.e. the first $n_{z}$ modal states) to be only excited by themselves. That is, we want $\psi_{j}[k]$ and $\psi_{j+1}[k]$ to be only excited by $\psi_{j}[k]$ and $\psi_{j+1}[k]$ for $j=1,3, \ldots, 2 n_{i}-1$ and $\psi_{j}[k]$ to be only excited by $\psi_{j}[k]$ for $j=2 n_{i}+1,2 n+i+2, \ldots, n_{z}$. As a first step, the choices

$$
\hat{\boldsymbol{f}}_{l}=\mathbf{0} \quad l=n_{z}+1, n_{z}+2, \ldots, n_{c} .
$$

are made, so that the last $n_{c}-n_{z}$ modal states do not affect the first $n_{z}$ modal states. Next, it is desired that

$$
\begin{array}{lll}
\boldsymbol{\gamma}_{\psi, j}^{\mathrm{T}} \hat{\boldsymbol{f}}_{l}=\mathbf{0} & j=1,3, \ldots, 2 n_{i}-1 & l=1,2, \ldots, n_{z}, l \neq j, l \neq j+1 \\
\boldsymbol{\gamma}_{\psi, j}^{\mathrm{T}} \hat{\boldsymbol{f}}_{l}=\mathbf{0} & j=2,4, \ldots, 2 n_{i} & l=1,2, \ldots, n_{z}, l \neq j, l \neq j-1
\end{array}
$$

holds, so that the modal states associated with the complex eigenvalues are only excited by themselves. Similarly, we want

$$
\boldsymbol{\gamma}_{\psi, j}^{\mathrm{T}} \hat{\boldsymbol{f}}_{l}=0 \quad j=2 n_{i}+1,2 n_{i}+2, \ldots, n_{z} \quad l=1,2, \ldots, n_{z}, l \neq j
$$

for the modal states associated with the real unstable eigenvalues. If we achieve (4.14) and (4.15) and use (4.13), we have

$$
\begin{aligned}
\psi_{j}[k+1] & =\left(\delta_{j}-\gamma_{\psi, j}^{\mathrm{T}} \hat{\boldsymbol{f}}_{j}\right) \psi_{j}[k]+\left(\omega_{j}-\boldsymbol{\gamma}_{\psi, j}^{\mathrm{T}} \hat{\boldsymbol{f}}_{j+1}\right) \psi_{j+1}[k]+\boldsymbol{\psi}_{y, j} \boldsymbol{y}[k+r] \\
\psi_{j+1}[k+1] & =-\left(\omega_{j}+\boldsymbol{\gamma}_{\psi, j+1}^{\mathrm{T}} \hat{\boldsymbol{f}}_{j}\right) \psi_{j}[k]+\left(\delta_{j}-\boldsymbol{\gamma}_{\psi, j+1} \hat{\boldsymbol{f}}_{j+1}\right) \psi_{j+1}[k]+\boldsymbol{\psi}_{y, j+1} \boldsymbol{y}[k+r]
\end{aligned}
$$

for $j=1,3, \ldots, 2 n_{i}-1$, and

$$
\psi_{j}[k+1]=\left(\lambda_{j}-\gamma_{\psi, j} \hat{\boldsymbol{f}}_{j}\right) \psi_{j}[k]+\boldsymbol{\gamma}_{y, j} \boldsymbol{y}[k+r] \quad j=2 n_{i}+1,2 n i+2, \ldots, n_{z}
$$

for the first $n_{z}$ states. Note that the first $n_{z}$ states of the closed-loop system $\boldsymbol{\Lambda}-\boldsymbol{\Gamma}_{\psi} \hat{\mathbf{F}}$ are in canonical modal form as well, so that the first $n_{z}$ columns of $\hat{\mathbf{F}}$ 
allow us to place the corresponding eigenvalues. The complex eigenvalues of the closed-loop system have to be conjugate-complex again, so we want (from (4.16))

$$
\begin{aligned}
\delta_{j}-\gamma_{\psi, j}^{\mathrm{T}} \hat{\boldsymbol{f}}_{j} \stackrel{!}{=} \delta_{j}-\gamma_{\psi, j+1} \hat{\boldsymbol{f}}_{j+1} & \Rightarrow \boldsymbol{\gamma}_{\psi, j}^{\mathrm{T}} \hat{\boldsymbol{f}}_{j}-\boldsymbol{\gamma}_{\psi, j+1} \hat{\boldsymbol{f}}_{j+1}=0 \\
\omega_{j}-\gamma_{\psi, j}^{\mathrm{T}} \hat{\boldsymbol{f}}_{j+1} \stackrel{!}{=} \omega_{j}+\gamma_{\psi, j+1}^{\mathrm{T}} \hat{\boldsymbol{f}}_{j} & \Rightarrow \quad \gamma_{\psi, j+1}^{\mathrm{T}} \hat{\boldsymbol{f}}_{j}+\boldsymbol{\gamma}_{\psi, j}^{\mathrm{T}} \hat{\boldsymbol{f}}_{j+1}=0
\end{aligned}
$$

for $j=1,3, \ldots, 2 n_{i}-1$. This means that for the $i$ th conjugate-complex eigenvalue pair $\left(i=1,2, \ldots, n_{i}\right)$ in modal form we need a block

$$
\left[\begin{array}{cc}
\delta_{i, \text { closed-loop }} & \omega_{i, \text { closed-loop }} \\
-\omega_{i, \text { closed-loop }} & \delta_{i, \text { closed-loop }}
\end{array}\right]
$$

with (naturally) $\delta_{i, \text { closed-loop }}=\delta_{i, \text { closed-loop }}$ and $\omega_{i, \text { closed-loop }}=\omega_{i, \text { closed-loop }}$

Moreover, we want the closed-loop system to be stable, so we demand

$$
\left|\left(\delta_{j}-\boldsymbol{\gamma}_{\psi, j}^{\mathrm{T}} \hat{\boldsymbol{f}}_{j}\right) \pm j\left(\omega_{j}-\boldsymbol{\gamma}_{\psi, j}^{\mathrm{T}} \hat{\boldsymbol{f}}_{j+1}\right)\right|<1 \quad j=1,3, \ldots, 2 n_{i}-1 .
$$

and

$$
\left|\lambda_{j}-\gamma_{\psi, j} \hat{\boldsymbol{f}}_{j}\right|<1 \quad j=2 n_{i}+1,2 n_{i}+2, \ldots, n_{z}
$$

Finally, it has to be examined how the addition of $\hat{\mathbf{F}}$ has influenced the remaining $n_{c}-n_{z}$ eigenvalues (with (4.13) we only ensured that the last $n_{c}-n_{z}$ modal states do not affect the first $n_{z}$ modal states; the first $n_{z}$ modal states, however, do have an influence on the last $n_{c}-n_{z}$ modal states). In order to do so, we first state that we can partition

$$
\Lambda=\left[\begin{array}{cc}
\Lambda_{1} & 0 \\
0 & \Lambda_{2}
\end{array}\right]
$$

where $\boldsymbol{\Lambda}_{1}$ and $\boldsymbol{\Lambda}_{2}$ are matrices in canonical modal form containing the unstable and stable eigenvalues of $\widetilde{\boldsymbol{\Phi}}_{f}$, respectively (if generalized eigenvectors have been used, $\boldsymbol{\Lambda}_{2}$ additionaly has ones above the main diagonal). Together with the other results from this section, we can write

$$
\boldsymbol{\psi}[k+1]=\underbrace{\left[\begin{array}{ll}
\hat{\boldsymbol{\Lambda}}_{1} & \hat{\boldsymbol{\Lambda}}_{2} \\
\hat{\boldsymbol{\Lambda}}_{3} & \hat{\boldsymbol{\Lambda}}_{4}
\end{array}\right]}_{=\boldsymbol{\Lambda}-\boldsymbol{\Gamma}_{\psi} \hat{\mathbf{F}}} \boldsymbol{\psi}[k]+\boldsymbol{\Gamma}_{y} \boldsymbol{y}[k+r]
$$


for the transformed system with

$$
\begin{aligned}
& \hat{\boldsymbol{\Lambda}}_{1}=\left[\begin{array}{cc}
\hat{\boldsymbol{\Lambda}}_{1,1} & \mathbf{0} \\
\mathbf{0} & \hat{\boldsymbol{\Lambda}}_{1,2}
\end{array}\right] \\
& \hat{\boldsymbol{\Lambda}}_{1,1}=\operatorname{blkdiag}\left(\mathbf{A}_{1}, \mathbf{A}_{2}, \ldots, \mathbf{A}_{n_{i}}\right) \\
& \mathbf{A}_{i}=\left[\begin{array}{cc}
\delta_{2 i-1}-\gamma_{\psi, 2 i-1} \hat{\boldsymbol{f}}_{2 i-1} & \omega_{2 i-1}-\gamma_{\psi, 2 i-1} \hat{\boldsymbol{f}}_{2 i} \\
-\left(\omega_{2 i-1}-\gamma_{\psi, 2 i-1} \hat{\boldsymbol{f}}_{2 i}\right) & \delta_{2 i-1}-\boldsymbol{\gamma}_{\psi, 2 i-1} \hat{\boldsymbol{f}}_{2 i-1}
\end{array}\right] \quad i=1,2, \ldots, n_{i} \\
& \hat{\boldsymbol{\Lambda}}_{1,2}=\left[\begin{array}{lll}
\lambda_{2 n_{i}+1}-\gamma_{\psi, 2 n_{i}+1} \hat{\boldsymbol{f}}_{2 n_{i}+1} & & \\
& \ddots & \\
& & \lambda_{n_{z}}-\boldsymbol{\gamma}_{\psi, n_{z}} \hat{\boldsymbol{f}}_{n_{z}}
\end{array}\right] \\
& \hat{\Lambda}_{2}=\mathbf{0} \\
& \hat{\boldsymbol{\Lambda}}_{3}=\left[\begin{array}{ccc}
-\gamma_{\psi, n_{z}+1} \hat{\boldsymbol{f}}_{1} & \ldots & -\boldsymbol{\gamma}_{\psi, n_{z}+1} \hat{\boldsymbol{f}}_{n_{z}} \\
\vdots & \ddots & \vdots \\
-\gamma_{\psi, n_{c}} \hat{\boldsymbol{f}}_{1} & \cdots & -\boldsymbol{\gamma}_{\psi, n_{c}} \hat{\boldsymbol{f}}_{n_{z}}
\end{array}\right] \\
& \hat{\Lambda}_{4}=\Lambda_{2} \text {. }
\end{aligned}
$$

This summarizes the whole approach. Because $\hat{\boldsymbol{\Lambda}}_{2}=\mathbf{0}$ in $(4.24 \mathrm{e})$, the eigenvalues

of the closed-loop system are the eigenvalues of $\hat{\boldsymbol{\Lambda}}_{1}$ and $\hat{\boldsymbol{\Lambda}}_{4}(\hat{\boldsymbol{\Lambda}}$ is a block tridiagonal matrix). We can place the eigenvalues in $\hat{\Lambda}_{1}$ by the choice of the first $n_{z}$ columns of $\hat{\mathbf{F}}$, so we can make sure that we move the unstable eigenvalues (which derive from the non-minimum phase zeros) into the unit circle. Moreover, because of $(4.24 \mathrm{~g})$, the remaining $n_{c}-n_{z}$ eigenvalues are the stable eigenvalues of $\widetilde{\boldsymbol{\Phi}}_{f}$ (hence we have not changed them by introducing $\boldsymbol{v}[k])$.

In conclusion, by introducing feedback of the form $\boldsymbol{v}[k]=\mathbf{F} \boldsymbol{x}_{c}[k]$ to the ideal control law (3.18) and by designing the feedback gain matrix according to this section, we are able to move all unstable eigenvalues of the filter into the unit circle and keep the already stable eigenvalues in their respective spot.

\subsubsection{Design by Optimization}

The idea of this section is to include the equality demands (4.14), (4.15), (4.18) and the inequality demands (4.21), (4.20) (which ensure that the the inverse filter 
is stabilized) for the design of the feedback gain matrix $\mathbf{F}$ into an optimization problem so that the norm of the resulting $\mathbf{F}$ is minimized to limit its influence on the ideal inversion control law. Therefore, an optimization problem was formulated to design $\mathbf{F}$ and which includes the stability demands (4.21), (4.20) as inequality constraints. Further, the demands (4.14), (4.15), (4.18) are included as equality constraints.

The gain matrix $\mathbf{F}$ is obtained after designing $\hat{\mathbf{F}}$ (according to (4.8e)):

$$
\mathbf{F}=-\hat{\mathbf{F}} \mathbf{W}
$$

As design parameters, we have the $p \cdot n_{z}$ entries of the first $n_{z}$ columns of $\hat{\mathbf{F}}$ (because we set the last $n_{c}-n_{z}$ columns of $\hat{\mathbf{F}}$ to zero in $\left.(4.13)\right)$, so we define

$$
\boldsymbol{x}=\left[\begin{array}{c}
\hat{\boldsymbol{f}}_{1} \\
\vdots \\
\hat{\boldsymbol{f}}_{n_{z}}
\end{array}\right]
$$

as the decision variable (please note that $\boldsymbol{x}$ in this case does not refer to any state variable). Thus we define

$$
\min _{\boldsymbol{x} \in \mathbb{R}^{p n_{z}}}\|\hat{\mathbf{F}} \mathbf{W}\|_{F}^{2}
$$

as the optimization problem, together with the inequality constraints (4.21) and (4.20) and the equality constraints (4.14), (4.15) and (4.18). The squared norm of $\mathbf{F}$ is minimized because it simplifies the calculations later on.

This optimization problem with nonlinear constraints can be either solved with Matlab's fmincon function, or some further analysis can be done to solve it with the Lagrange dualism, which is what will be done here.

The Lagrange dualism is very briefly introduced here, see for example [38] for 
details. Consider an optimization problem in standard form

$$
\begin{array}{cl}
\operatorname{minimize} & f_{0}(\boldsymbol{x}) \\
\text { subject to } & f_{i}(\boldsymbol{x}) \leq 0, \quad i=1, \ldots, m \\
& h_{i}(\boldsymbol{x})=0, \quad i=1, \ldots, p
\end{array}
$$

with decision variable $\boldsymbol{x} \in \mathbb{R}^{n}$, and it is assumed that its domain $\mathcal{D}$ is nonempty (please note that the notation here is independent of the usual notation in this thesis: $n, m$ and $p$ in (4.28) do not refer to the plant order or to the number of plant inputs or outputs). By defining the Lagrangian $L: \mathbb{R}^{n} \times \mathbb{R}^{m} \times \mathbb{R}^{p} \rightarrow \mathbb{R}$ the constraints are taken into consideration:

$$
L(\boldsymbol{x}, \boldsymbol{\mu}, \boldsymbol{\nu})=f_{0}(\boldsymbol{x})+\sum_{i=1}^{m} \mu_{i} f_{i}(\boldsymbol{x})+\sum_{i=1}^{p} \nu_{i} h_{i}(\boldsymbol{x})=f_{0}(\boldsymbol{x})+\boldsymbol{\mu}^{\mathrm{T}} \boldsymbol{f}(\boldsymbol{x})+\boldsymbol{\nu}^{\mathrm{T}} \boldsymbol{h}(\boldsymbol{x})
$$

with

$$
\begin{aligned}
\boldsymbol{f}(\boldsymbol{x}) & =\left[\begin{array}{lll}
f_{1}(\boldsymbol{x}) & \ldots & f_{m}(\boldsymbol{x})
\end{array}\right]^{\mathrm{T}} \\
\boldsymbol{h}(\boldsymbol{x}) & =\left[\begin{array}{lll}
h_{1}(\boldsymbol{x}) & \ldots & h_{p}(\boldsymbol{x})
\end{array}\right]^{\mathrm{T}} \\
\boldsymbol{\mu} & =\left[\begin{array}{lll}
\mu_{1} & \ldots & \mu_{m}
\end{array}\right]^{\mathrm{T}} \\
\boldsymbol{\nu} & =\left[\begin{array}{lll}
\nu_{1} & \ldots & \nu_{p}
\end{array}\right]^{\mathrm{T}} .
\end{aligned}
$$

We refer to $\mu_{i}$ as the Lagrange multiplier associated with the $i$-th inequality constraint $f_{i}(\boldsymbol{x}) \leq 0$; similarly we refer to $\nu_{i}$ as the Lagrange multiplier associated with the $i$-th equality constraint $h_{i}(\boldsymbol{x})=0$. Furthermore, we define the Lagrange dual function $g: \mathbb{R}^{m} \times \mathbb{R}^{p} \rightarrow \mathbb{R}$ as the minimum value of the Lagrangian over $\boldsymbol{x}$ :

$$
g(\boldsymbol{\mu}, \boldsymbol{\nu})=\inf _{\boldsymbol{x} \in \mathcal{D}} L(\boldsymbol{x}, \boldsymbol{\mu}, \boldsymbol{\nu})=\inf _{\boldsymbol{x} \in \mathcal{D}}\left(f_{0}(\boldsymbol{x})+\boldsymbol{\mu}^{\mathrm{T}} \boldsymbol{f}(\boldsymbol{x})+\boldsymbol{\nu}^{\mathrm{T}} \boldsymbol{h}(\boldsymbol{x})\right)
$$

The dual function yields lower bounds on the optimal value $p^{*}$ of the primal problem (4.28):

$$
g(\boldsymbol{\mu}, \boldsymbol{\nu}) \leq p^{*}
$$


so the question is what the best lower bound is that can be obtained by the parameters $\boldsymbol{\mu}, \boldsymbol{\nu}$. This leads to the optimization problem

$$
\begin{array}{ll}
\operatorname{maximize} & g(\boldsymbol{\mu}, \boldsymbol{n u}) \\
\text { subjet to } & \boldsymbol{\mu} \geq \mathbf{0}
\end{array}
$$

where $\boldsymbol{\mu} \geq \mathbf{0}$ means that all elements of the vector $\boldsymbol{\mu}$ should be $\geq 0$. This problem is called the Lagrange dual problem associated with the primal problem (4.28). It is a convex optimization problem because the objective to be maximized over is concave and the constraint is convex. This is the case whether the primal problem (4.28) is convex or not [38].

Next, we want to find an expression for the Lagrange dual function for our problem at hand. The details of the calculations and the definitions of the following matrices are described in Appendix A. It is first noted that the primal problem (4.27) can be expressed as:

minimize $\boldsymbol{x}^{\mathrm{T}} \mathbf{Y} \boldsymbol{x}$

subject to $\boldsymbol{x}^{\mathrm{T}}\left(\mathbf{U}_{j}^{\mathrm{T}} \mathbf{S}_{j} \mathbf{U}_{j}+\mathbf{U}_{j+1}^{\mathrm{T}} \mathbf{S}_{j} \mathbf{U}_{j+1}\right) \boldsymbol{x}-2 \boldsymbol{\epsilon}_{j}^{\mathrm{T}} \boldsymbol{x}+r_{j}<0 \quad i=1,3, \ldots, 2 n_{i}-1$,

$$
\mathrm{MN} \boldsymbol{x}+\boldsymbol{k}<\mathbf{0}
$$

$$
\mathrm{G} \boldsymbol{x}=\mathbf{0}
$$

where the details can be found in Appendix A. After some calculations (see Appendix A for a detailed discussion) the Lagrangian can be written as

$$
L(\boldsymbol{x}, \boldsymbol{\mu}, \boldsymbol{\nu})=\boldsymbol{x}^{\mathrm{T}} \mathbf{Q}(\boldsymbol{\mu}) \boldsymbol{x}+\left(\boldsymbol{\mu}^{\mathrm{T}} \mathbf{P}+\boldsymbol{\nu}^{\mathrm{T}} \mathbf{G}\right) \boldsymbol{x}+\boldsymbol{\mu}^{\mathrm{T}} \boldsymbol{d}
$$

The notation $\mathbf{Q}(\boldsymbol{\mu})$ denotes that the matrix $\mathbf{Q}$ is not constant, but depends on the Lagrangian multiplier $\boldsymbol{\mu}$. It can be shown that $\mathbf{Q}$ is positive definite. Thus, the dual function becomes

$$
g(\boldsymbol{\mu}, \boldsymbol{\nu})=\inf _{\boldsymbol{x} \in \mathbb{R}^{p n_{z}}} L(\boldsymbol{x}, \boldsymbol{\mu}, \boldsymbol{\nu})=\inf _{\boldsymbol{x} \in \mathbb{R}^{p n_{z}}}\left(\boldsymbol{x}^{\mathrm{T}} \mathbf{Q} \boldsymbol{x}+\left(\boldsymbol{\mu}^{\mathrm{T}} \mathbf{P}+\boldsymbol{\nu}^{\mathrm{T}} \mathbf{G}\right) \boldsymbol{x}\right)+\boldsymbol{\mu}^{\mathrm{T}} \boldsymbol{d} .
$$


and a minimum is obtained when (4.36) is differentiated and the derivative is set to zero, since $\mathbf{Q}$ is positive definite. It follows:

$$
\underbrace{\left(\mathbf{Q}+\mathbf{Q}^{\mathrm{T}}\right)}_{:=\widetilde{\mathbf{Q}}} \boldsymbol{x}^{*}+\left(\mathbf{P}^{\mathrm{T}} \boldsymbol{\mu}+\mathbf{G}^{\mathrm{T}} \boldsymbol{\nu}\right) \stackrel{!}{=} \mathbf{0} \Rightarrow \boldsymbol{x}^{*}=-\widetilde{\mathbf{Q}}^{-1}\left(\mathbf{P}^{\mathrm{T}} \boldsymbol{\mu}+\mathbf{G}^{\mathrm{T}} \boldsymbol{\nu}\right)
$$

where $\widetilde{\mathbf{Q}}=\mathbf{Q}+\mathbf{Q}^{\mathrm{T}}$ was defined. Plugging this result back into (4.36) yields

$g(\boldsymbol{\mu}, \boldsymbol{\nu})=L\left(\boldsymbol{x}^{*}, \boldsymbol{\mu}, \boldsymbol{\nu}\right)=\left(\boldsymbol{\mu}^{\mathrm{T}} \mathbf{P}+\boldsymbol{\nu}^{\mathrm{T}} \mathbf{G}\right)\left(\widetilde{\mathbf{Q}}^{-1} \mathbf{Q} \widetilde{\mathbf{Q}}^{-1}-\widetilde{\mathbf{Q}}^{-1}\right)\left(\mathbf{P}^{\mathrm{T}} \boldsymbol{\mu}+\mathbf{G}^{\mathrm{T}} \boldsymbol{\nu}\right)+\boldsymbol{\mu}^{\mathrm{T}} \boldsymbol{d}$

for the dual function, where the two facts

$$
\begin{aligned}
\left(\widetilde{\mathbf{Q}}^{-1}\right)^{\mathrm{T}} & =\left(\widetilde{\mathbf{Q}}^{\mathrm{T}}\right)^{-1} \\
\widetilde{\mathbf{Q}}^{\mathrm{T}} & =\widetilde{\mathbf{Q}}
\end{aligned}
$$

were used [34]. Thus we have

$$
\max _{\boldsymbol{\mu} \geq \mathbf{0}}\left(\boldsymbol{\mu}^{\mathrm{T}} \mathbf{P}+\boldsymbol{\nu}^{\mathrm{T}} \mathbf{G}\right)\left(\widetilde{\mathbf{Q}}^{-1} \mathbf{Q} \widetilde{\mathbf{Q}}^{-1}-\widetilde{\mathbf{Q}}^{-1}\right)\left(\mathbf{P}^{\mathrm{T}} \boldsymbol{\mu}+\mathbf{G}^{\mathrm{T}} \boldsymbol{\nu}\right)+\boldsymbol{\mu}^{\mathrm{T}} \boldsymbol{d}
$$

as the (convex) dual optimization problem. Since we can express any maximization problem as a minimization problem

$$
\max _{\boldsymbol{x} \in \mathcal{D}} f(\boldsymbol{x})=\min _{\boldsymbol{x} \in \mathcal{D}}-f(\boldsymbol{x})
$$

we define

$$
\hat{\mathbf{Q}}=\widetilde{\mathbf{Q}}^{-1}-\widetilde{\mathbf{Q}}^{-1} \mathbf{Q} \widetilde{\mathbf{Q}}^{-1}
$$

so we finally obtain

$$
\min _{\boldsymbol{\mu} \geq \mathbf{0}}\left(\boldsymbol{\mu}^{\mathrm{T}} \mathbf{P}+\boldsymbol{\nu}^{\mathrm{T}} \mathbf{G}\right) \hat{\mathbf{Q}}\left(\mathbf{P}^{\mathrm{T}} \boldsymbol{\mu}+\mathbf{G}^{\mathrm{T}} \boldsymbol{\nu}\right)-\boldsymbol{\mu}^{\mathrm{T}} \boldsymbol{d}
$$

as the dual minimization problem that we want to solve using Matlab's fmincon function. We have to use the fmincon function as we still have to consider a constrained optimization problem. 
Once the optimization problem (4.43) is solved, and we have obtained $\boldsymbol{\mu}^{*}$ and $\boldsymbol{\nu}^{*}$ as its solutions, we can conclude

$$
\boldsymbol{x}^{*}=-\widetilde{\mathbf{Q}}^{-1}\left(\mathbf{P}^{\mathrm{T}} \boldsymbol{\mu}^{*}+\mathbf{G}^{\mathrm{T}} \boldsymbol{\nu}^{*}\right)
$$

by using (4.37), hence the entries of $\hat{\mathbf{F}}$ (and therefore $\mathbf{F}$ as well) are known.

\subsubsection{Discussion of the Approach}

This approach to directly influence the unstable eigenvalues of the inverse filter (which derive from the plant's non-minimum phase zeros) was introduced and developed for several reasons. First, the transformation to modal canonical form offers the possibility to express the eigenvalues of the closed-loop inverse filter in terms of the decision variable $\boldsymbol{x}$ (i.e. the entries of $\mathbf{F}$ ), so that the objective function and the constraints can be analytically used to find the Lagrange dual function (and therefore it enables us to let Matlab solve a convex optimization problem). Moreover, if $\mathbf{F}$ is designed using "classic" pole-placement techniques, multiple problems arise. It is not clear how the filter eigenvalues have to chosen to obtain a satisfying tracking performance, and a $d l q r$ approach requires an iterative design to find adequate weights.

It turned out, however, that this optimization approach has some disadvantages, especially in the implementation. For instance, the original unstable inverse

filter state-transition matrix $\widetilde{\boldsymbol{\Phi}}_{f}$ has a multiple eigenvalue in 0 (see Section 3.2 .1 ), making it harder to transform the matrix to canonical modal form, since generalized eigenvectors have to be used. The transformation matrix $\mathbf{V}$ has a high condition number, and the entries in its inverse $\mathbf{V}^{-1}=\mathbf{W}$ are relatively large. It is argued then that it becomes difficult to minimize the Frobenius norm $\|\hat{\mathbf{F}} \mathbf{W}\|_{F}$ if $\mathbf{W}$ has very large entries. It is noted that if the hybrid approach approach mentioned in Section 3.2.3 is applied, the problem with the multiple eigenvalue vanishes and this approach yields better results than before, but they are still not 
a real improvement to the classic design of adding advances until the inverse filter is stabilized, and the derivation and implementation are much more complicated.

It is concluded that the success of this approach does not only depend on the non-minimum phase zeros of the plant, but also on how "well" the inverse filter can be transformed to canonical modal form. Furthermore, the chosen objective function (i.e. the Frobenius norm of the resulting controller F) may not be ideal to obtain a "good" precision tracking system. Despite this disappointing conclusion, this approach played an important role in this thesis, since the work on this initial idea to involve optimization in the design process of $\mathbf{F}$ led to more thoughts on how optimization can improve tracking and how a corresponding optimization problem has to be formulated. This resulted in the approach presented in Section 4.3.

\subsection{Frequency Optimization Approach}

This section focuses on the idea of optimizing the frequency response of the cascade of the inverse filter and the system whose output is desired to be tracked. In theory, the filter-based tracking system should be a pure delay of $d$ samples $(d \geq r)$, i.e. the ideal frequency response of the cascade is given by

$$
\mathbf{G}_{\text {ideal }}=e^{-j \omega d T} \mathbf{I}_{p}
$$

Let $\mathbf{H}_{c}\left(e^{j \omega T}\right)$ denote the frequency response of the system, and $\mathbf{H}_{f}\left(e^{j \omega T}\right)$ the frequency response of the inverse filter with feedback matrix, $\mathbf{F}$ (defined in (3.56)). Assume that a grid of frequency points $\omega_{k}, k=1, \ldots, N$ is given, based on the frequencies included in the system. In the simulation results presented in Chapter $5, \omega_{N}$ was chosen to be the maximum frequency for which none of the magnitudes of the main diagonal elements of the feedback tracking system shown in Fig. 3.1 has dropped by $3 \mathrm{~dB}$, and $\omega_{1}=0 \frac{\mathrm{rad}}{\mathrm{s}}$ (dc gain). 
According to (4.45), an initial idea for the optimization could be $\mathbf{F}^{*}=\arg \min _{\mathbf{F}} \sum_{k=1}^{N}\left\|\mathbf{H}_{c}\left(e^{j \omega_{k} T}\right) \mathbf{H}_{f}\left(e^{j \omega_{k} T}\right)-e^{-j \omega_{k} d T} \mathbf{I}_{p}\right\|_{\mathrm{F}}^{2} \quad$ s.t. $\quad \widetilde{\boldsymbol{\Phi}}_{f}+\boldsymbol{\Gamma}_{c} \mathbf{F}$ is stable

where $\|\cdot\|_{\mathrm{F}}$ denotes the Frobenius norm. As a quick reminder, $\widetilde{\boldsymbol{\Phi}}_{f}$ denotes the unstable state-transition matrix of the inverse filter that approximately inverts the system with a delay of $d$ samples, and to which the feedback gain matrix $\mathbf{F}$ has not yet been added (inversion is exact for $d=r$ ). This initial optimization problem, even though it is a formulation of what we want to achieve, can prove to be quite wasteful in practice. The reason for that is that the optimization, for a MIMO system, attempts to optimize the off-diagonal elements of $\mathbf{H}_{c}\left(e^{j \omega T}\right) \mathbf{H}_{f}\left(e^{j \omega T}\right)$ as well (i.e. zero magnitude and phase over all frequencies), but this is not necessarily required for a good inversion-based tracking performance. The phase of the offdiagonal elements is not important for the tracking performance if the attenuation is small enough, so the demand for the off-diagonal elements is to "simply" provide a sufficient attenuation over the frequency range of interest.

The following sections deal with different formulation for the objective function of the optimization problem that were considered in this thesis. For better readability, a few definitions are introduced first. Define $z_{k}=e^{j \omega_{k} T}$. Introduce

$$
\mathbf{G}\left(z_{k}\right)=\mathbf{H}_{c}\left(z_{k}\right) \mathbf{H}_{f}\left(z_{k}\right)
$$

and let the elements of $\mathbf{G}\left(z_{k}\right)$ be denoted by $g_{i j}\left(z_{k}\right), i, j=1, \ldots, p$ :

$$
\mathbf{G}\left(z_{k}\right)=\left[\begin{array}{ccc}
g_{11}\left(z_{k}\right) & \ldots & g_{1 p}\left(z_{k}\right) \\
\vdots & \ddots & \vdots \\
g_{p 1}\left(z_{k}\right) & \ldots & g_{p p}\left(z_{k}\right)
\end{array}\right] .
$$

\subsubsection{Main Diagonal Elements}

The main diagonal elements are the main concern of the optimization. For a good tracking performance, the frequency responses $g_{i i}\left(z_{k}\right), i=1, \ldots, p$ have to be 
"close" to the ideal frequency response $g_{\text {ideal }}\left(z_{k}\right)=e^{-j \omega d T}$. Therefore, the following cost function is introduced for the main diagonal elements

$$
\begin{aligned}
J_{\text {main }} & =\sum_{k=1}^{N}\left\|\left[\begin{array}{c}
g_{11}\left(z_{k}\right)-e^{-j \omega_{k} d T} \\
\vdots \\
g_{p p}\left(z_{k}\right)-e^{-j \omega_{k} d T}
\end{array}\right]\right\|_{2}^{2}=\sum_{k=1}^{N} \sum_{i=1}^{p}\left(g_{i i}^{*}\left(z_{k}\right)-e^{j \omega_{k} d T}\right)\left(g_{i i}\left(z_{k}\right)-e^{-j \omega_{k} d T}\right) \\
& =\sum_{k=1}^{N} \sum_{i=1}^{p}\left|g_{i i}\left(z_{k}\right)-e^{-j \omega_{k} d T}\right|^{2}
\end{aligned}
$$

where $g_{i i}^{*}\left(z_{k}\right)$ denotes the conjugate-complex of $g_{i i}\left(z_{k}\right)$. It optimizes the distance between the actual frequency response $g_{i i}\left(z_{k}\right)$ and the desired value in the complex plant over the frequencies of interest.

\subsubsection{Off-Diagonal Elements}

Two approaches were considered for the off-diagonal elements. Since we only care about the attenuation, the first cost function tries to minimize the squared magnitudes of the off-diagonal elements:

$$
J_{\text {off }}=\alpha \sum_{k=1}^{N} \sum_{i=1}^{p} \sum_{\substack{j=1 \\ j \neq i}}^{p}\left|g_{i j}\left(z_{k}\right)\right|^{2}
$$

The factor $\alpha$ can be used to weight the cost function for the off-diagonal elements in comparison to the cost function for the main diagonal elements. Naturally, the weighting factor could also be different for every $k, i$ and $j$, so a factor $\alpha_{k i j}$ would be possible, if weighting for different frequencies and elements is desired.

Another possibility is to define a desired attenuation for the off-diagonal elements. First, let the element $g_{i j}\left(z_{k}\right)$ be given by

$$
g_{i j}\left(z_{k}\right)=a_{i j}\left(z_{k}\right)+j b_{i j}\left(z_{k}\right) .
$$

For $i \neq j$, an attenuation of

$$
\left|g_{i j}\left(z_{k}\right)\right|=\sqrt{a_{i j}^{2}\left(z_{k}\right)+b_{i j}^{2}\left(z_{k}\right)} \stackrel{!}{\leq} R_{a}
$$


is demanded. It can bee seen that $g_{i j}\left(z_{k}\right)$ has to lie within a circle with radius $R_{a}$ in the complex plane, centered in the origin. An idea for a penalty function (see [39]) is given by

$$
J_{\text {off }}=\sum_{k=1}^{N} \sum_{i=1}^{p} \sum_{\substack{j=1 \\ j \neq i}}^{p} e^{\alpha\left(\sqrt{a_{i j}^{2}\left(z_{k}\right)+b_{i j}^{2}\left(z_{k}\right)}-R_{a}\right)} .
$$

The argument of the exponential function was chosen such that it is negative if $g_{i j}\left(z_{k}\right)$ lies within the desired circle, and positive if it is not. Together with an adequate choice of the weighting factor $\alpha, g_{i j}\left(z_{k}\right)$ has a very high contribution to the penalty function if it is outside the desired circle (i.e. if the attenuation at this frequency is higher than desired), and it has basically no contribution if the attenuation demand is satisfied. It goes without saying that $\alpha$ and $R_{a}$ could be chosen differently for every $k, i$ and $j$, if so desired.

\subsubsection{Stability Constraint}

As indicated in (4.46), the optimization has to be constrained. Namely, the

feedback gain matrix $\mathbf{F}$ has to stabilize $\widetilde{\boldsymbol{\Phi}}_{f}$, i.e. the eigenvalues of the matrix $\widetilde{\boldsymbol{\Phi}}_{f}+\boldsymbol{\Gamma}_{c} \mathbf{F}$ must be all located inside the unit circle. Usually, constraint functions $f(\boldsymbol{x})$ must be defined such that they return a negative value if the constraint is satisfied and a positive value otherwise. In our case, one possibility for the constraint function is to consider the spectral radius $\rho$ of the matrix $\boldsymbol{\Phi}_{f}=\widetilde{\boldsymbol{\Phi}}_{f}+\boldsymbol{\Gamma}_{c} \mathbf{F}$. It is defined as the largest absolute value of the eigenvalues of the matrix [34]. Hence, the constraint function could be formulated as

$$
f(\mathbf{F})=\rho\left(\widetilde{\boldsymbol{\Phi}}_{f}+\boldsymbol{\Gamma}_{c} \mathbf{F}\right)-1
$$

Alternatively, a constraint function for every eigenvalue of the filter could be defined:

$$
f_{i}(\mathbf{F})=\left|\lambda_{i}\right|-1, \quad i=1, \ldots, n_{c}
$$

where $\lambda_{i}$ denotes the $i$ th eigenvalue of $\widetilde{\boldsymbol{\Phi}}_{f}+\boldsymbol{\Gamma}_{c} \mathbf{F}$ and $n_{c}$ is the order of the filter. 
Another possibility is to not explicitly constrain the optimization, but to include the stability constraint in the objective function. In order to do so, a penalty function is designed, similar to Section 4.3.2. The desired area in the complex plane, in which the eigenvalues must be kept in, is the unit circle. If $\lambda_{i}=\delta_{i}+j \omega_{i}, i=1, \ldots, n_{c}$, then

$$
J_{\text {eig }}=\sum_{i=1}^{n_{c}} e^{\beta\left(\sqrt{\delta_{i}^{2}+\omega_{i}^{2}}-1\right)}
$$

is a reasonable penalty function for the stability constraint. As before, the eigenvalue $\lambda_{i}$ has a very high contribution if it is outside the unit circle, and hardly any contribution otherwise. To ensure a successful design, the parameter $\beta$ should be assigned a pretty high value (e.g. $\left.\beta=10^{4}\right)$.

\subsubsection{Discussion of the Approach}

In theory, this frequency optimization approach can be used in an attempt to get a sufficient tracking performance within the frequency range of interest, while only using the minimum number of $r$ advances to invert the system, i.e. the cascade of the filter and the closed-loop system is an (approximate) delay of $r$ samples. For most cases, however, the optimization will not be able to find a $\mathbf{F}$ which stabilizes the filter while also maintaining a respectable tracking performance over the desired frequency range, if only $r$ advances are used. That being the case, it is recommended to use a "hybrid approach" between adding delays and designing the feedback matrix $\mathbf{F}$ (via optimization) to stabilize the filter, discussed at the end of Section 3.2.3. Accordingly, the system matrices for the inverse filters are given in (3.56), with $\widetilde{\mathbf{C}}_{f}$ and $\widetilde{\mathbf{D}}_{f}$ being defined in (3.62). A reasonable procedure to find the optimal inverse filter design is to let $s$ run from 0 to $s_{\max }$ and use the number of advances $s$ that produced the smallest value of the objective function.

As an initial value $\mathbf{F}_{0}$, a result from the classical feedback design techniques, presented in Section 4.1, can be used. In particular, it proved to be useful to 
determine $\mathbf{F}_{0}$ via a $d l q r$ design. 


\section{CHAPTER 5}

\section{Example Systems and Simulation Results}

In this chapter, the example systems that were examined in the course of this thesis will be presented, together with the tracking results obtained from simulations in Matlab. The desired reference trajectory will be denoted by $\boldsymbol{r}[k]$, the actual output by $\boldsymbol{y}[k]$.

The feedback matrix $\mathbf{K}_{d}=\left[\begin{array}{ll}\mathbf{K}_{1} & -\mathbf{K}_{2}\end{array}\right]$ for the tracking system in Fig. 3.1 was designed according to the guidelines in [27]. These rules use normalized Bessel poles, which can be found in Table 5.1 [25, 27].

As a rule of thumb, the sampling time $T$ to discretize continuous-time systems can be chosen with respect to the desired settling time $T_{S}$. If $n$ denotes the order of the continuous-time plant and $n_{a}$ the order of the additonal dynamics (see Section $3.1)$, then

$$
T \approx \frac{T_{S}}{20\left(n+n_{a}\right)}
$$

was often used as a rule of thumb in this thesis.

An important performance measure for the tracking systems in Fig. 3.5 and Fig. 3.6 is the precision tracking bandwidth $\omega_{b}$. If all the frequencies included in the reference trajectory lie within the bandwidth $0 \leq \omega \leq \omega_{b}$, it is assumed that the

\begin{tabular}{cl} 
Variable & Pole Locations \\
\hline$s_{1}$ & -4.6200 \\
$s_{2}$ & $-4.0530 \pm j 2.3400$ \\
$s_{3}$ & $-5.0093,-3.9668 \pm j 3.7845$ \\
$s_{4}$ & $-4.0156 \pm j 5.0723,-5.5281 \pm j 1.6553$ \\
$s_{5}$ & $-6.4480,-4.1104 \pm j 6.3142,-5.9268 \pm j 3.0813$ \\
$s_{6}$ & $-4.2169 \pm j 7.5300,-6.2613 \pm j 4.4018,-7.1205 \pm j 1.4540$ \\
\hline
\end{tabular}

Table 5.1: Normalized Bessel poles for 1st through 6th-order systems with 1-second settling time. To get a settling time of $T_{S}$ seconds, divide all poles by $T_{S}$. 
architectures are able to perfectly track this reference. Let $A_{i j}(j \omega)=\left|g_{i j}\left(e^{j \omega T}\right)\right|$ be the magnitude and $\varphi_{i j}(j \omega)=\angle g_{i j}\left(e^{j \omega T}\right)$ be the phase of the frequency response of the transfer function from the $j$ th input to the $i$ th output of the entire filterbased tracking system (shown in Fig. 3.5 and Fig. 3.6, respectively). Assume that we choose a pure sinusoid

$$
r_{i}[k]=\sin (\omega k T)
$$

with frequency $\omega$ as the reference signal for every output, $i=1, \ldots, p$. The difference between the ideal output and the actual output will be used to formulate a condition for the precision tracking bandwidth.

As a first step, we derive the output of the precision tracking system for the chosen reference input. With the presented notation, we obtain

$$
y_{i}[k]=\sum_{j=1}^{p} A_{i j}(j \omega) \sin \left(\omega k T+\varphi_{i j}(j \omega)\right) \quad i=1 \ldots, p
$$

as the steady-state response of the $i$ th ouput [26]. According to Sections 3.3 and 3.4 , the cascade of the inverse filter and the system is an approximate delay of $d$ samples on every output signal. The ideal frequency response of the tracking system (see also Section 4.3) is therefore

$$
\mathbf{G}_{\text {ideal }}=e^{-j \omega d T} \mathbf{I}_{p}
$$

and hence the ideal output for the chosen reference input is

$$
y_{\text {ideal }, i}[k]=\sin \left(\omega k T+\varphi_{0}(j \omega)\right) \quad i=1 \ldots, p
$$

with

$$
\varphi_{0}(j \omega)=-\omega d T
$$

The tracking error $\boldsymbol{\delta}[k]$ will be in this case defined as the difference between the ideal output $\boldsymbol{y}_{\text {ideal }}[k]$ and the actual output $\boldsymbol{y}[k]$, so for the tracking error of the 
ith output

$$
\delta_{i}[k]=y_{\text {ideal }, i}[k]-y_{i}[k]=\sin \left(\omega k T+\varphi_{0}(j \omega)\right)-\sum_{j=1}^{p} A_{i j}(j \omega) \sin \left(\omega k T+\varphi_{i j}(j \omega)\right) .
$$

follows. Obviously, $\boldsymbol{\delta}[k]$ is a (discrete) function of time, $t=k T$, but we want to derive a measure for the tracking error at a specific frequency $\omega$, independent of the time $t$, which we will call $\boldsymbol{\epsilon}(j \omega)$. For this reason, we define

$\epsilon_{i}(j \omega):=\max _{k}\left\{\delta_{i}[k]\right\}=\max _{k}\left\{\sin \left(\omega k T+\varphi_{0}(j \omega)\right)-\sum_{j=1}^{p} A_{i j}(j \omega) \sin \left(\omega k T+\varphi_{i j}(j \omega)\right)\right\}$

as the performance measure for every output. Next, Euler's formula

$$
e^{j x}=\cos (x)+j \sin (x)
$$

will be used to find an expression for $\boldsymbol{\epsilon}(j \omega)[40]^{1}$. This is done by rewriting (5.7) (for ease of notation and better readability $k T$ is replaced by $t$ and the $\omega$-dependency of the magnitudes and the phase shifts is omitted):

$$
\begin{aligned}
\sin \left(\omega t+\varphi_{0}\right)-\sum_{j=1}^{p} A_{i j} \sin \left(\omega t+\varphi_{i j}\right) & =\operatorname{Im}\left\{e^{j\left(\omega t+\varphi_{0}\right)}\right\}-\sum_{j=1}^{p} \operatorname{Im}\left\{A_{i j} e^{j\left(\omega t+\varphi_{i j}\right)}\right\} \\
=\operatorname{Im}\left\{e^{j\left(\omega t+\varphi_{0}\right)}-\sum_{j=1}^{p} A_{i j} e^{j\left(\omega t+\varphi_{i j}\right)}\right\} & =\operatorname{Im}\{e^{j \omega t} \underbrace{\left(e^{j \varphi_{0}}-\sum_{j=1}^{p} A_{i j} e^{j \varphi_{i j}}\right)}_{:=\hat{A}_{i} e^{j \hat{\varphi}_{i}}}\} \\
=\operatorname{Im}\left\{\hat{A}_{i} e^{j \varphi_{i}} e^{j \omega t}\right\} & =\operatorname{Im}\left\{\hat{A}_{i} e^{j\left(\omega t+\varphi_{i}\right)}\right\} \\
& =\hat{A}_{i} \sin \left(\omega t+\hat{\varphi}_{i}\right) .
\end{aligned}
$$

In the light of this observation, it can be concluded that $\delta_{i}[k]$ is a sinusoid with amplitude $\hat{A}_{i}$ and phase $\hat{\varphi}_{i}$, so $\epsilon_{i}(j \omega)$ is defined as the amplitude of this sinusoid.

\footnotetext{
${ }^{1}$ https://ccrma.stanford.edu/ jos/filters/Sum_Sinusoids_Same_Frequency.html
} 
Since the amplitude $\hat{A}_{i}$ is given as the magnitude of the complex number $z=$ $e^{j \varphi_{0}}-\sum_{j=1}^{p} A_{i j} e^{j \varphi_{i j}}$, it can be expressed as

$$
\begin{aligned}
\operatorname{Re}(j \omega) & =\cos \left(\varphi_{0}(j \omega)\right)-\sum_{j=1}^{p} A_{i j}(j \omega) \cos \left(\varphi_{i j}(j \omega)\right) \\
\operatorname{Im}(j \omega) & =\sin \left(\varphi_{0}(j \omega)\right)-\sum_{j=1}^{p} A_{i j}(j \omega) \sin \left(\varphi_{i j}(j \omega)\right) \\
\epsilon_{i}(j \omega) & =\sqrt{(\operatorname{Re}(j \omega))^{2}+(\operatorname{Im}(j \omega))^{2}}
\end{aligned}
$$

where Euler's formula was used again.

Finally, we define the precision tracking bandwidth $\omega_{b}$ to be the largest value $\omega$ for which $\epsilon_{i}(j \omega)<K_{b}$ holds for every output, $i=1, \ldots, p$. For the following simulation results we chose $K_{b}=10^{-2}$, i.e.

$$
\omega_{b}=\text { largest } \omega \text { s.t. } \epsilon_{i}(\omega)<10^{-2}, \quad i=1, \ldots, p \text {. }
$$

The precision tracking bandwidth will for example be used to evaluate how much of the reference trajectory's energy is included in this bandwidth. In order to do so, we introduce (with $\Omega_{k}=\omega_{k} T$ )

$$
R\left(j \Omega_{k}\right)=R[k]=\sum_{n=0}^{N-1} r[n] W_{N}^{k n}
$$

as the discrete Fourier transformation (see for example [41]) of the reference input $r[k]$, where $N$ is the number of samples of $r[k]$ and

$$
W_{N}=e^{-j \frac{2 \pi}{N}}
$$

holds. The value $R[k]$ occurs at the frequency $\omega_{k}=\frac{2 \pi k}{N T}$ with $0 \leq k<N$. According to Parseval's relation, we have

$$
E=\sum_{n=0}^{N-1}|r[n]|^{2}=\frac{1}{N} \sum_{n=0}^{N-1}|R[n]|^{2}
$$

as the energy contained in the reference trajectory [41]. Since the DFT is periodic and $R[-k]=R^{*}[k]$ holds $[41]$, we also have $R[N-k]=R^{*}[k]$. Therefore, the 
amount of the signal's energy that is contained up to the frequency $\omega_{k} \leq \frac{\pi}{T}$ is calculated as

$$
E_{k}=\frac{|R(j 0)|^{2}+2 \cdot \sum_{n=1}^{k}\left|R\left(j \Omega_{n}\right)\right|^{2}}{\sum_{n=0}^{N-1}\left|R\left(j \Omega_{n}\right)\right|^{2}}
$$

in the following sections.

Moreover, two other performance measures were established in the thesis and will be taken into account for the example systems in the following sections. These performance measures were taken from [4], where the tracking of continuous-time SISO systems is discussed. One performance measure $\left(J_{e}\right)$ is introduced to quantify the energy in the tracking error, another one $\left(J_{m}\right)$ to quantify the peak deviation from the desired trajectory. Assume that $y_{i}[k]$ denotes the $i$ th output and $r_{i}[k]$ the corresponding desired trajectory. Then, we can define

$$
J_{e, i}=\int\left\{\left(\left(y_{i}[k]-r_{i}[k]\right) \cdot w[k]\right)^{2}\right\} d t
$$

and

$$
J_{m, i}=\max _{k}\left\{\left(\left(y_{i}[k]-r_{i}[k]\right) \cdot w_{i}[k]\right)^{2}\right\}
$$

as the respective performance measures for one output, where $w_{i}[k]$ is a weighting function defined by

$$
w_{i}[k]= \begin{cases}\frac{1}{\left|r_{i}[k]\right|} & \text { if }\left|r_{i}[k]\right| \geq r_{i, t} \\ \frac{1}{r_{i, t}} & \text { otherwise }\end{cases}
$$

and $r_{i, t}>0$ denotes a threshold value for the $i$ th output. In the following, it is set to

$$
r_{i, t}=0.1 \cdot \max _{k}\left|r_{i}[k]\right|,
$$

i.e. $10 \%$ of the maximum absolute value of the reference trajectory for the $i$ th output. If the reference for the $i$ th output is $r_{i}[k]=0$ for all $k$, it is set to $r_{i, t}=1$. The weighting function was introduced so that the tracking error $y_{i}[k]-r_{i}[k]$ is 
normalized and becomes comparable for different systems. However, if the reference input is zero or close to zero, the performance measures would become very large if they were divided by $r_{i}[k]$, and therefore the threshold $r_{i, t}$ was introduced. Matlab's trapz function was used to carry out the numerical integration in (5.17).

For MIMO systems, the performance measures for the single outputs will be combined, so that

$$
J_{e}=\frac{1}{p} \sum_{i=1}^{p} J_{e, i}
$$

and

$$
J_{m}=\max _{i} J_{m, i}
$$

will be used as the performance measures in the following sections.

Another measure that will be discussed is the (absolute) tracking error $\delta_{i}[k], i=1, \ldots, p$ for every output; that is $\delta_{i}[k]=y_{i}[k]-r_{i}[k]$. This is not to be confused with the stability robustness bounds $\delta_{1}$ and $\delta_{2}$ introduced in Section 3.1, as they will also be mentioned in the discussions and evaluations of the example systems.

The "standard" approach to calculate the CSF and IMP in the following sections will be the design presented in Section 3.2.2, i.e. advances will be added until all the eigenvalues of the inverse filter are contained in the chosen pole radius $R$. This result will then be contrasted and compared with a design that involves a feedback gain matrix $\mathbf{F}$ in order to stabilize the inverse filter. Most of the time a hybrid approach between adding advances and designing a feedback matrix will yield the best result.

Please note that all the Bode plots shown in the following sections are for the closed-loop system shown in Fig. 3.5 and Fig. 3.6, respectively. Thus, they are not indicative of gain or phase margin of the control system. 


\subsection{H-Frame System}

In this section, an H-Frame $X Y$ positioning system, which consists of two stationary motors, eight pulleys and a single drive belt, is considered. A detailed description of the system modeling and an 8th-order state-space model can be found in [42]. The system has two inputs and two outputs. The inputs are the voltages to the two motor power amplifiers, and the tracked outputs are the $x$ and $y$ positions of the cart.

The choice of the closed-loop poles of the feedback tracking system will be based on the pole and zero locations of the continuous-time plant. It has no finite zeros, but the following poles:

$$
\begin{aligned}
& s_{1,2}=-20.0068 \pm j 329.0277 \quad s_{6}=-43.8959 \\
& s_{3,4}=-21.9751 \pm j 249.2963 s_{7,8}=0 \\
& s_{5}=-15.6059 \text {. }
\end{aligned}
$$

Together with the additional dynamics, which were chosen to be integrators (see Section 3.1), the design model in (3.1) is a 10th-order system. A settling time of $T_{S}=0.1 \mathrm{~s}$ is desired, so the sampling time was chosen to be $T=5 \times 10^{-4} \mathrm{~s}$ (see (5.1)). According to the rules in [27], damping was added to the complex plant poles $s_{1}$ to $s_{4}$, and the remaining six poles were chosen to be $\frac{s_{6}}{T_{S}}$ (see Table 5.1). These poles were mapped using the $\mathrm{ZOH}$ pole-mapping formula, $\lambda_{i}=e^{s_{i} T}$, and the algorithm from [27] was used to calculate the (discrete-time) feedback gain matrix $\mathbf{K}_{d}$. The attained input-multiplicative and input-feedback stability robustness bounds were $\delta_{1}=0.6490$ and $\delta_{2}=0.7395$, respectively. After $\mathbf{K}_{d}$ is calculated, the (digital) feedback tracking system (Fig. 3.1) can be formed. The 
poles are located at

$$
\begin{array}{ll}
\lambda_{1,2}=0.9640 \pm j 0.1600 & \lambda_{7,8}=0.9689 \pm j 0.0213 \\
\lambda_{3,4}=0.9696 \pm j 0.1212 & \lambda_{9,10}=0.9650 \pm j 0.0070 \\
\lambda_{5,6}=0.9784 \pm j 0.0369 &
\end{array}
$$

while

$$
\begin{array}{ll}
\eta_{1}=-9.8030 & \eta_{4}=-0.9945 \\
\eta_{2}=-9.8280 & \eta_{5}=-0.1002 \\
\eta_{3}=-0.9912 & \eta_{6}=-0.1006
\end{array}
$$

are the zeros of the tracking system, which coincides with the zeros of the discretized plant. Thus, the feedforward filters become unstable due to the presence of the non-minimum phase (sampling) zeros $\eta_{1}$ and $\eta_{2}$.

\section{Command Shaping Filter}

The CSF can invert the closed-loop system in Fig. 3.1 exactly with a delay of $r=2$ samples. Since the largest absolute value of the eigenvalues of the feedback tracking system is $\left|\lambda_{\max }\right|=0.9791$, the pole radius $R$ for the CSF becomes $R=$

0.9896 (see (3.51)). The CSF needs $s=14$ additional advances to move the eigenvalues of the filter within the pole radius $R$, so the cascade of filter and feedback tracking system is an approximate delay of $d=16$ samples.

The Bode plot of the entire precision tracking system (shown in Fig. 3.5) can be seen in Fig. 5.1. The Bode plot of the feedback tracking system (FTS), i.e. the system in Fig. 3.1, is shown is as well. The precison tracking bandwidth achieved with the CSF is $\omega_{b}=149.5312 \frac{\mathrm{rad}}{\mathrm{s}}$.

Figures 5.2a and 5.2c show the tracking performance achieved with the CSF and $s=14$ additional delays. The chosen reference input causes the cart to move along a square whose sides have length $2.5 \mathrm{~cm}$. The corresponding absolute tracking errors $\delta_{i}=y_{i}-r_{1}, i=1,2$ can be seen in Figures $5.2 \mathrm{~b}$ and $5.2 \mathrm{~d}$. The 
(a) From input 1 to output 1
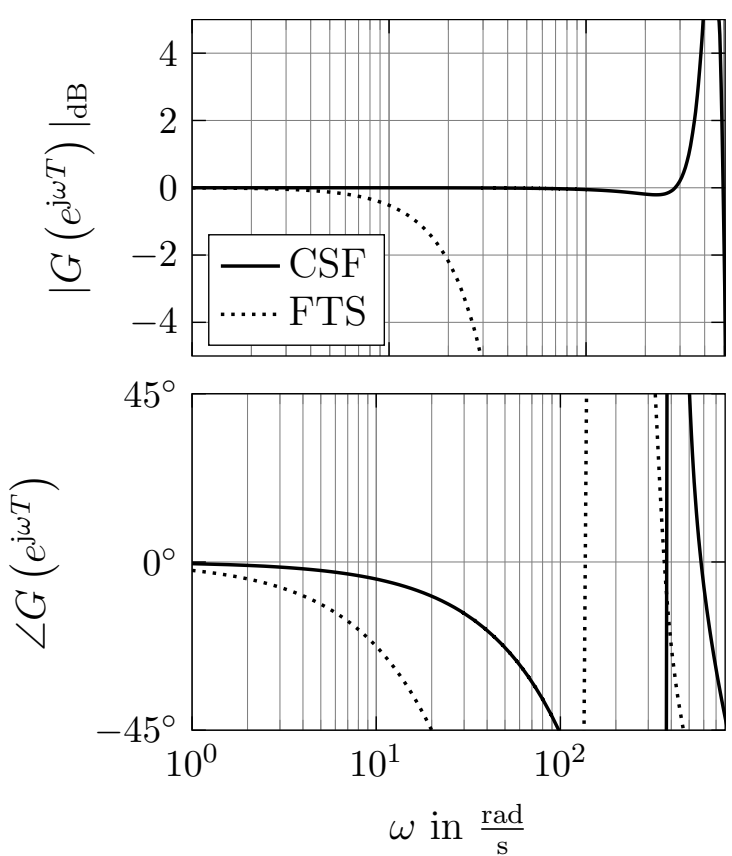

(c) From input 1 to output 2
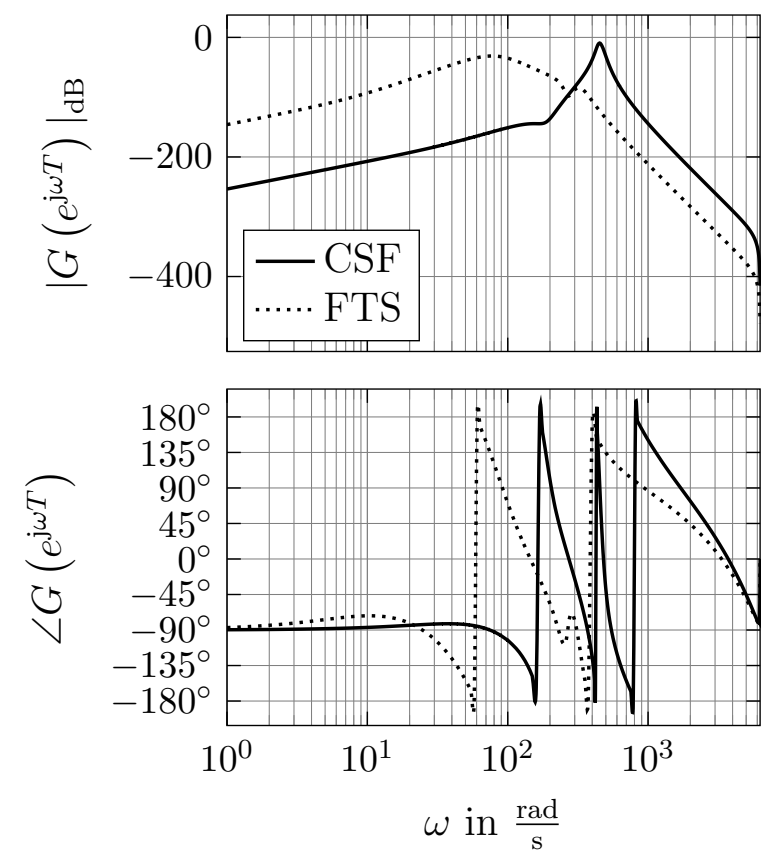

(b) From input 2 to output 1
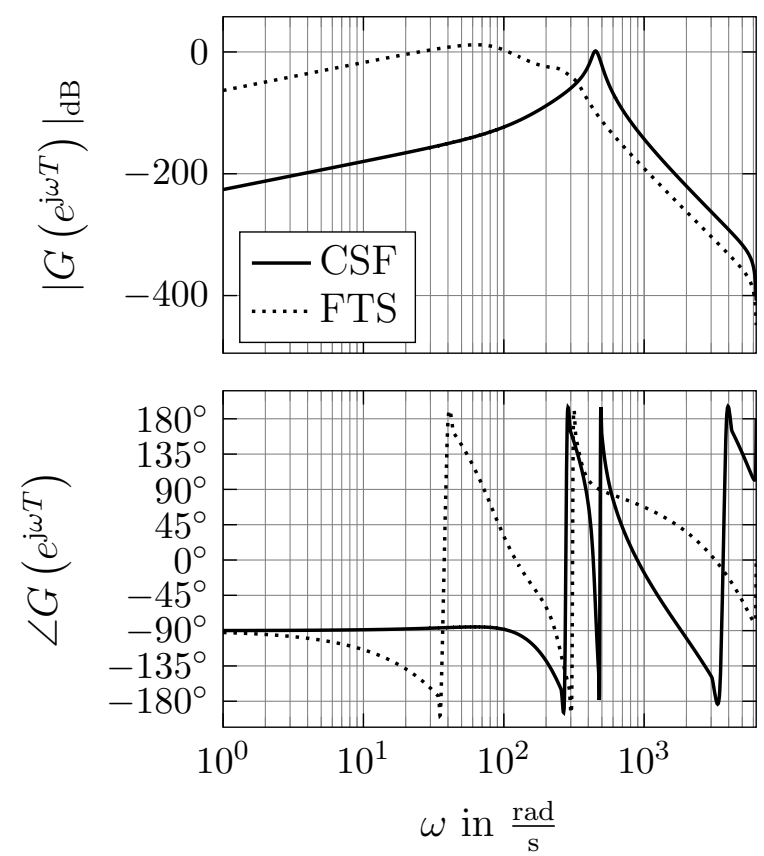

(d) From input 2 to output 2
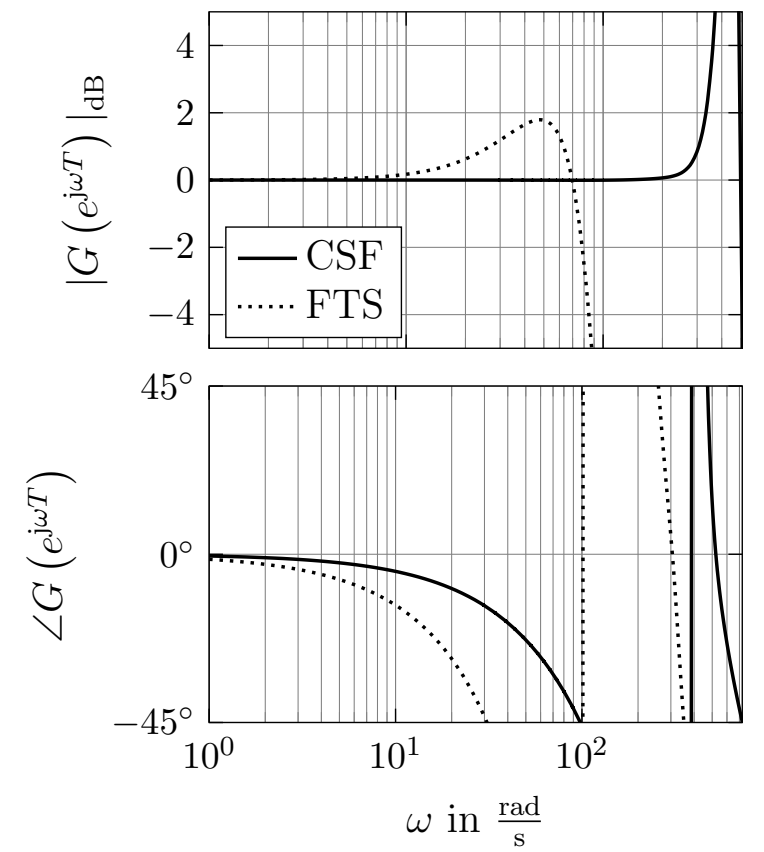

Figure 5.1: Frequency response of the H-Frame precision tracking system comparing a standard feedback tracking system (FTS) without feedforward, and the same feedback system with a feedforward CSF filter 
tracking error is never higher than $1 \times 10^{-3} \mathrm{~cm}$ for both outputs. The corresponding performance measures are $J_{e}=2.4659 \times 10^{-7}$ and $J_{m}=1.7671 \times 10^{-5}$, respectively. The achieved precision tracking bandwidth with this design contains almost 100\% of the reference input's energy.

It is possible to improve the tracking performance of the CSF if a hybrid approach between adding advances and the frequency optimization is used. For the H-Frame system, the unconstrained optimization, using (4.50) as penalty functions for the off-diagonal elements $(\alpha=10)$ and (4.56) as penalty function for the eigenvalue constraint $\left(\beta=1 \times 10^{4}\right)$, together with adding $s=9$ advances (on top of the $r$ advances already needed to derive the (unstable) CSF), gave the best result. The initial value $\mathbf{F}_{0}$ was determined using a $d l q r$ design, with $\mathbf{Q}=10^{-10} \cdot \mathbf{I}_{n_{c}}$ and $\mathbf{S}=100 \cdot \mathbf{I}_{p}$. The maximum frequency that was contained in the frequency grid was $\omega_{N}=91.9841 \frac{\mathrm{rad}}{\mathrm{s}}$.

After the optimization, the spectral radius of the CSF is $\rho\left(\boldsymbol{\Phi}_{f}\right)=0.9983$ and it reaches an objective function value of $J=8.9884 \times 10^{-7}$. Since $s=9$ additional advances were added, the cascade of the CSF and the closed-loop system is an approximate delay of $d=11$ samples (in contrast to the $d=16$ samples that were needed before). The actual frequency response of this CSF can be seen in Fig. 5.3. The result achieved with the frequency optimization approach (solid line) is contrasted with the result from "simply" adding $s=14$ delays (dotted line); the precision tracking bandwidth could be roughly doubled to $\omega_{b}=298.7422 \frac{\mathrm{rad}}{\mathrm{s}}$.

Figure 5.4 shows the tracking errors for the two outputs with the frequency optimization approach. While the tracking error $\delta_{2}$ stays roughly the same, the tracking error $\delta_{1}$ could be improved from $\delta_{1 \text {,old }}=1 \times 10^{-5} \mathrm{~m}$ to $\delta_{1, \text { new }}=4 \times 10^{-6} \mathrm{~m}$, i.e. by $\frac{\delta_{1, \text { old }}-\delta_{1, \text { new }}}{\delta_{1, \text { old }}}=60 \%$. This also shows in the performance measures, they could be further bettered to $J_{e}=2.9857 \times 10^{-8}$ and $J_{m}=2.8290 \times 10^{-6}$. 


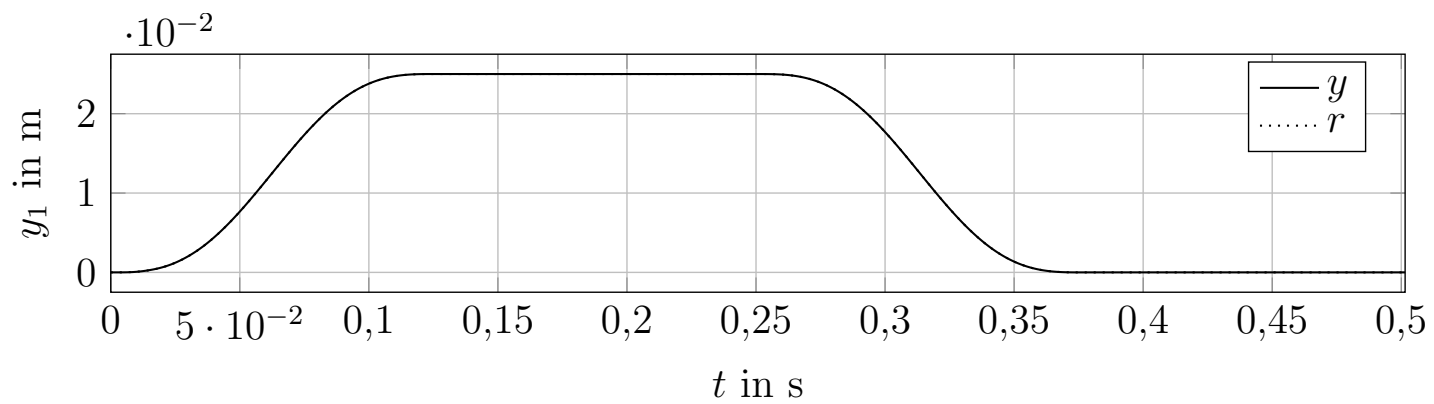

(a) Tracking of ouput 1

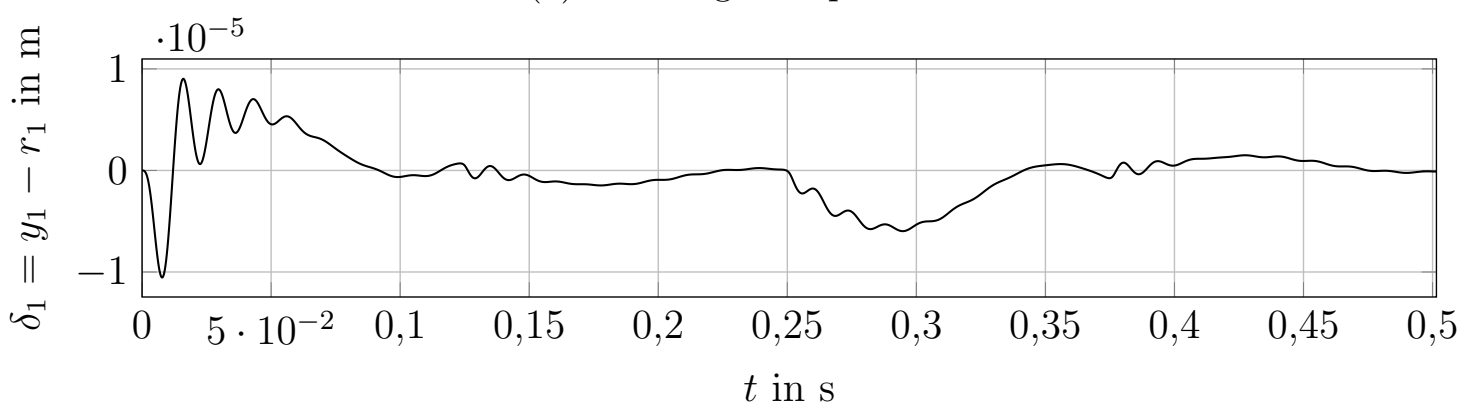

(b) Tracking error of ouput 1

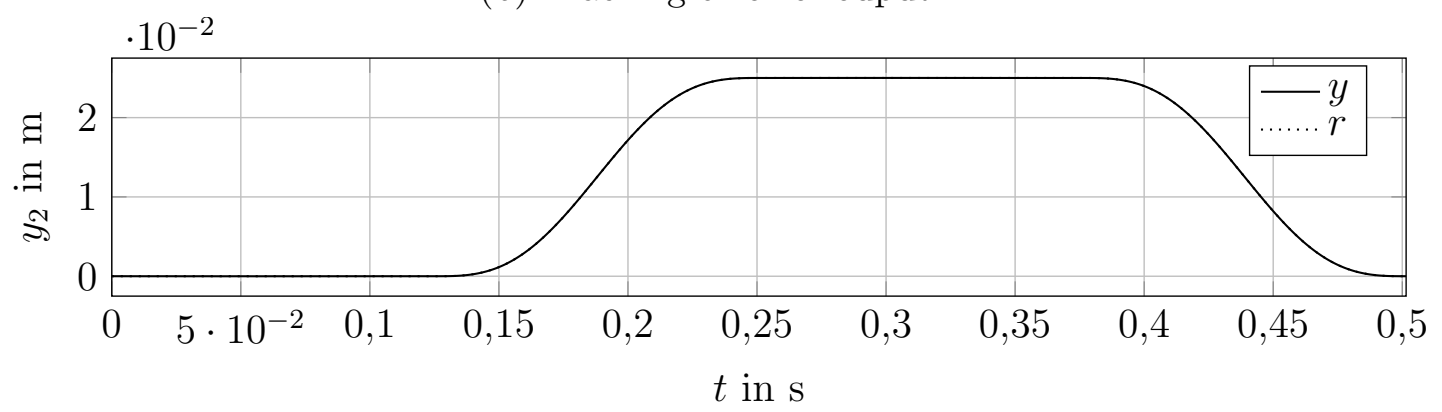

(c) Tracking of ouput 2

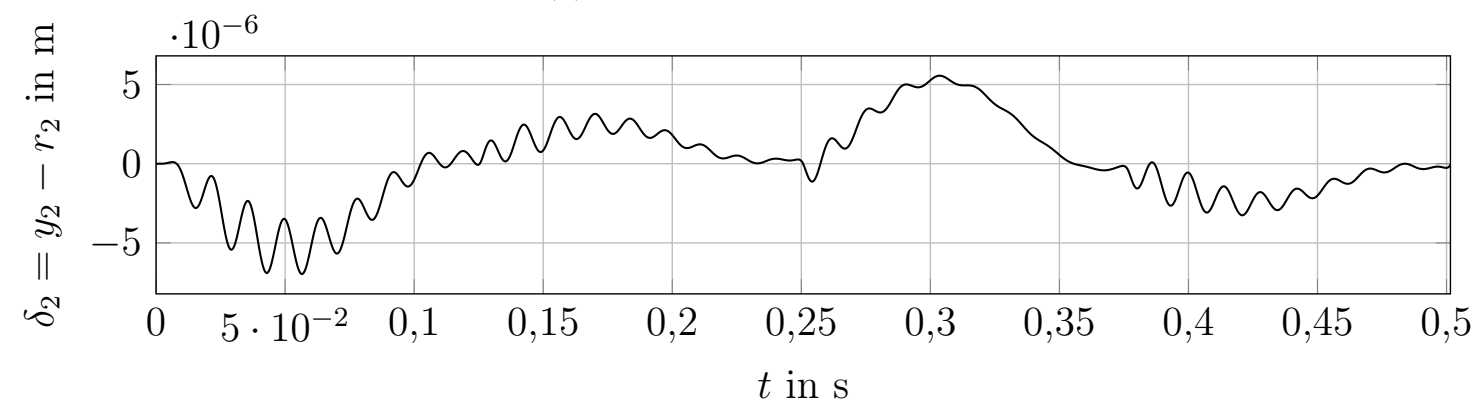

(d) Tracking error of output 2

Figure 5.2: H-Frame tracking (CSF) 
(a) From input 1 to output 1
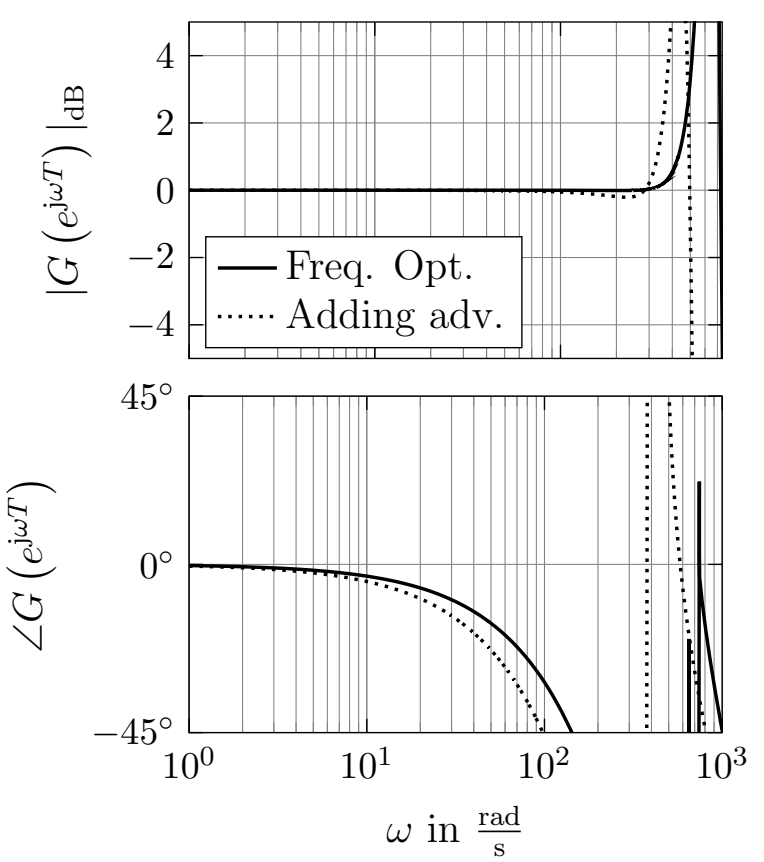

(c) From input 1 to output 2
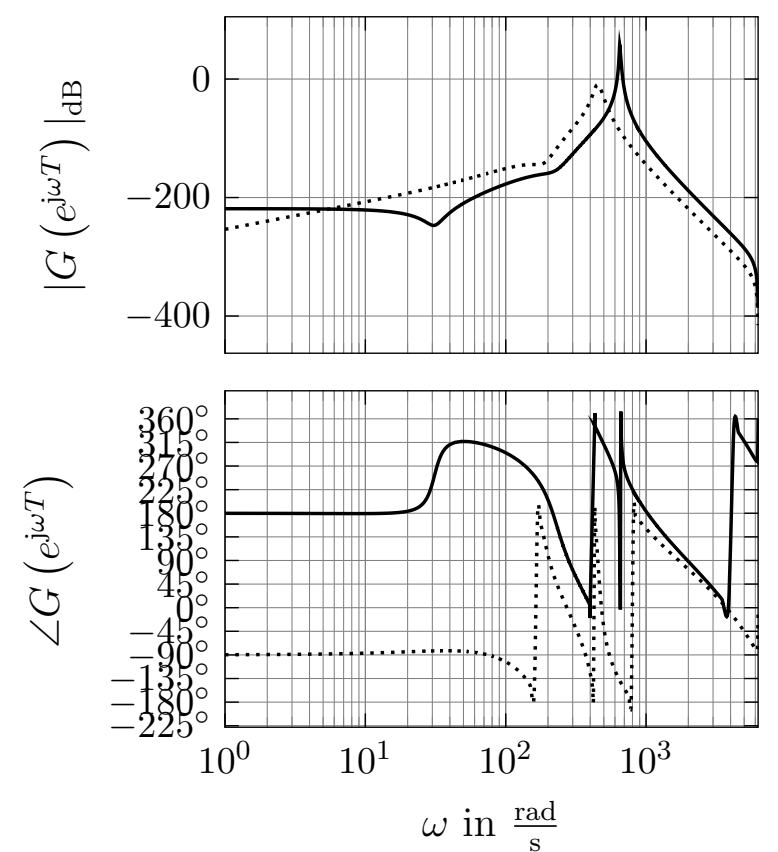

(b) From input 2 to output 1
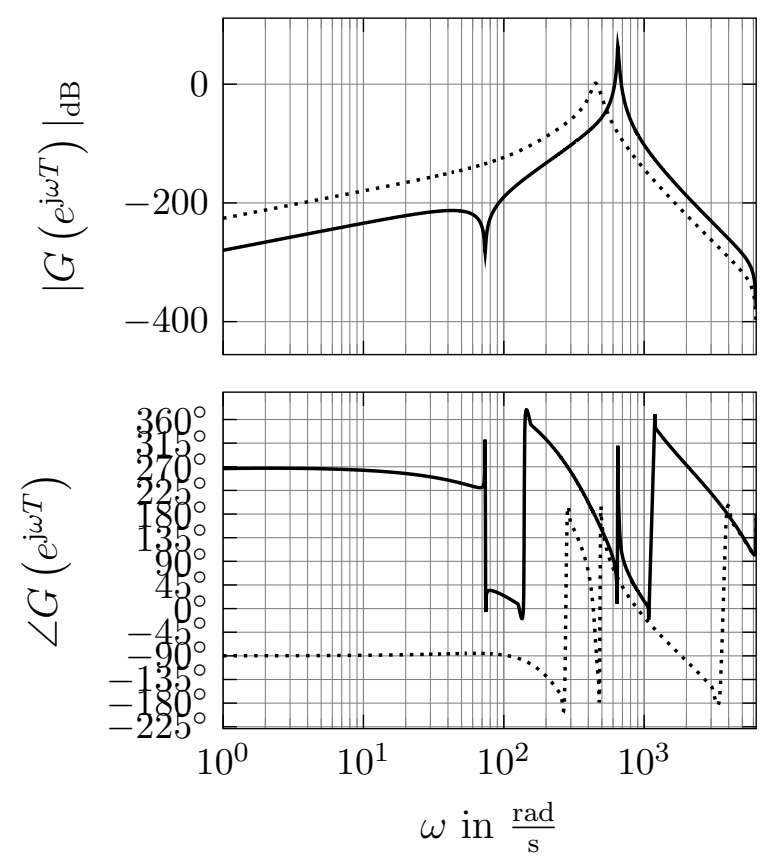

(d) From input 2 to output 2
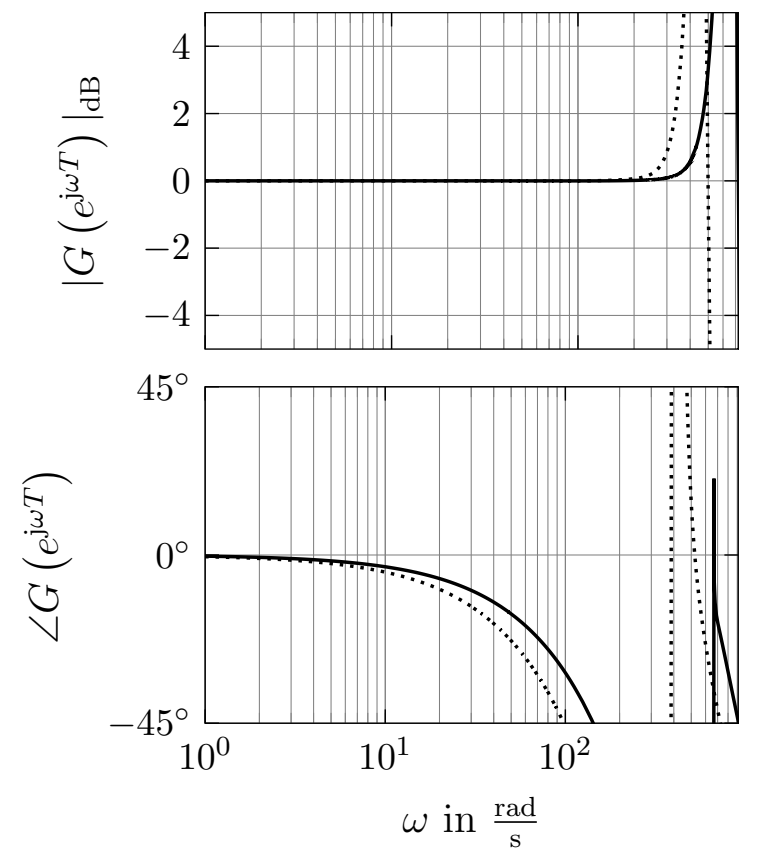

Figure 5.3: Frequency response of the H-Frame precision tracking system (CSF frequency optimization) 


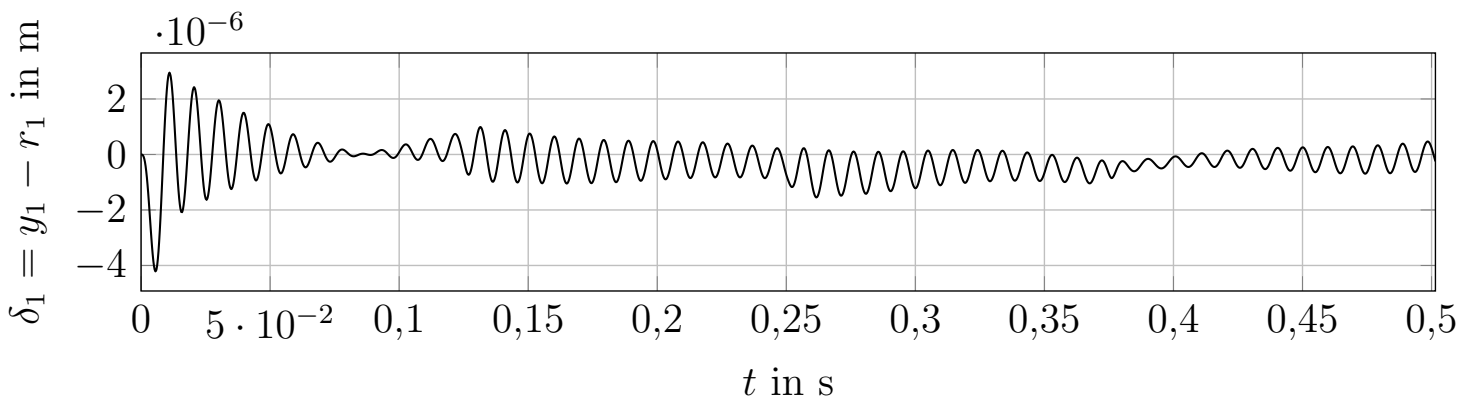

(a) Tracking error of ouput 1

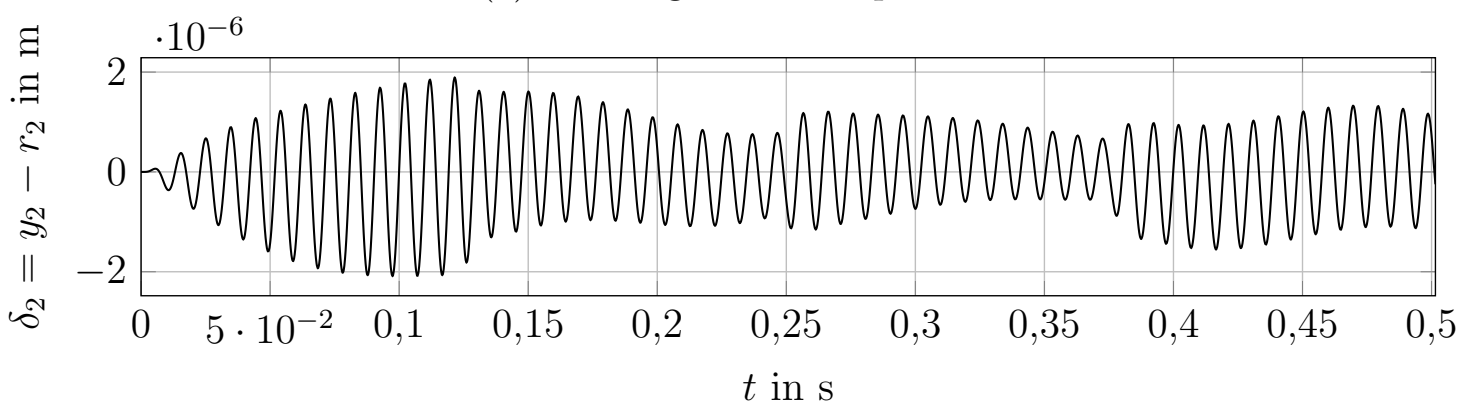

(b) Tracking error of output 2

Figure 5.4: H-Frame tracking (CSF - frequency optimization) 
Finally, it is noted that only cart and motor positions can be measured, but not the velocities, which are also included as states in the plant model. Therefore, an observer was added to validate the tracking performance if only the output $\boldsymbol{y}[k]$ is available for controlling the system (see Section 3.5 for details). The desired observer pole locations were calculated via a $d l q r$ design on the system $\left(\boldsymbol{\Phi}^{\mathrm{T}}, \mathbf{C}^{\mathrm{T}}\right)$, with weighting matrices $\mathbf{Q}=100 \cdot \mathbf{I}_{n}$ and $\mathbf{S}=\mathbf{I}_{p}$ (since the continuous-time plant does not have any zeros, the guidelines in [27] were not applied). The locations returned by this design were then used in the algorithm from [27], in order to find the observer gain $\mathbf{L}$ which ensures the desired pole locations and optimizes the stability robustness bounds. The resulting feedback tracking system suffers from a reduced stability robustness in comparison to the previous design, $\delta_{1}=0.4159$ and $\delta_{2}=0.3818$.

With this observer design, the spectral radius of $\boldsymbol{\Phi}_{c}$ increases to $\rho\left(\boldsymbol{\Phi}_{c}\right)=$ 0.9943, hence the pole radius becomes $R=0.9971$. The feedback tracking system can still be exactly inverted with a delay of $r=2$ samples, and $s=10$ additional delays are required to stabilize the CSF, making the tracking system an approximate delay of $d=12$ samples. The precision tracking bandwidth becomes $\omega_{b}=261.9769 \frac{\mathrm{rad}}{\mathrm{s}}$. The improvement in comparison to the standard CSF design without observer can be explained with the larger pole radius and the resulting fewer number of advances needed to keep the filter poles within this radius. If the same pole radius $(R=0.9971)$ is used without the observer, a similar precision tracking bandwidth is obtained. The resulting tracking errors of the CSF tracking architecture with an observer included are shown in Fig. 5.5. The absolute tracking error $\delta_{1}$ is better in comparison to the initial CSF result in Fig. 5.2, again due to the larger pole radius $R$ and the resulting fewer number of delays. In general it can be stated that the CSF architecture with an included observer is still able to 


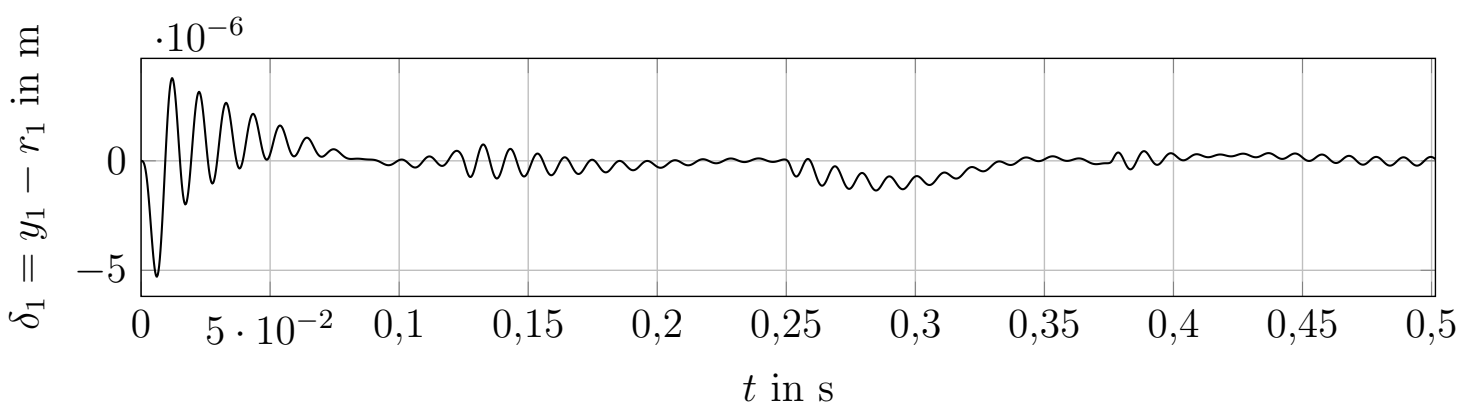

(a) Tracking error of ouput 1

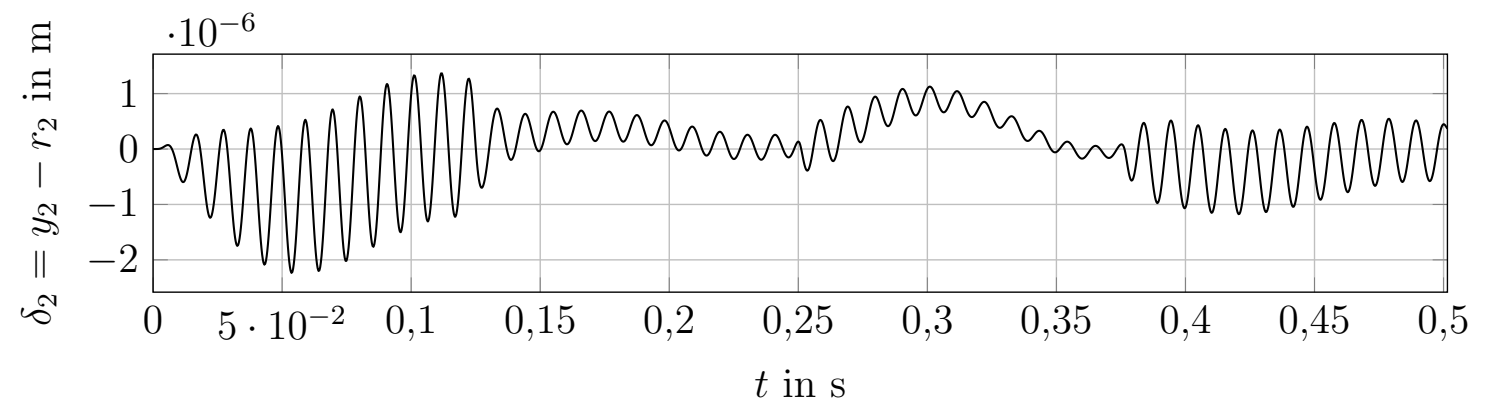

(b) Tracking error of output 2

Figure 5.5: H-Frame tracking (CSF - with observer)

track the reference trajectory.

As a next step, to validate the robustness of the tracking architecture, it is assumed that the system description is subject to uncertainties. The inverse filter design was based on the nominal plant model, but when the simulation was carried out, every parameter of the H-Frame system (see [42] for a description of the parameters) was changed by $10 \%$. The same observer was used as in the previous result. As to be expected, adding uncertainties substantially reduced the precision tracking bandwidth, we now have $\omega_{b}=3.1145 \frac{\mathrm{rad}}{\mathrm{s}}$. It has to be evaluated via a simulation if this bandwidth is sufficient to track the desired reference without visible tracking errors. The plot of the tracking performance and the corresponding tracking errors is shown in Fig. 5.6. Especially for the second output, the tracking error gets worse, as deviations between reference input $r_{2}[k]$ and plant output 
$y_{2}[k]$ in Fig. 5.6b are visible. Although this result is a decline in comparison to the previous results, it can still be argued that good tracking is achieved. The maximum absolute tracking error is $\left|\delta_{\max }\right|=0.9817 \mathrm{~mm}$, while the amplitude of the reference trajectory is $r_{\max }=2.5 \mathrm{~cm}$, so the tracking error is only around $4 \%$ of this amplitude. In addition, the performance measures are $J_{e}=0.0045$ and $J_{m}=0.1542$, which is considerably larger than the prior values, but still relatively small.

\section{Inverse Modified Plant}

The modified plant can be inverted exactly with a delay of $r=1$ sample. The algorithm needs $s=1$ more advances to stabilize the inverse filter, so that the cascade of the IMP and the modified plant is an approximate delay of $d=2$ samples. The spectral radius of the modified plant is $\rho\left(\Phi_{c}\right)=0.9803$, the pole radius becomes $R=0.9902$. The resulting Bode plot can be seen in Fig. 5.7, the result is contrasted with the result of the standard feedback tracking system. A clear improvement is noticed in comparison to the feedback tracking system. In particular, the IMP is able to greatly better the precision tracking bandwidth $\omega_{b}$; it obtains a value of $\omega_{b}=1.4551 \times 10^{3} \frac{\mathrm{rad}}{\mathrm{s}}$ which is more than the fourfold of the best CSF result. Additionally, the other performance measures shrink as well, the IMP reaches $J_{e}=1.4852^{-14}$ and $J_{m}=1.0782 \times 10^{-11}$. The plots of the tracking errors are shown in Fig. 5.8, and they reveal very high frequency oscillation in the tracking error at some time instances. These oscillations, however, have a very small amplitude. The tracking error is never higher than $\left|\delta_{\max }\right|=8.3 \mathrm{~nm}$ (which constitutes an improvement of $99.79 \%$ in comparison to the best CSF result).

In the IMP case, the frequency optimization approach yields very similar results in comparison to the standard design. An unconstrained optimization was solved with (4.50) as cost function for the off-diagonal elements, (4.56) as penalty 


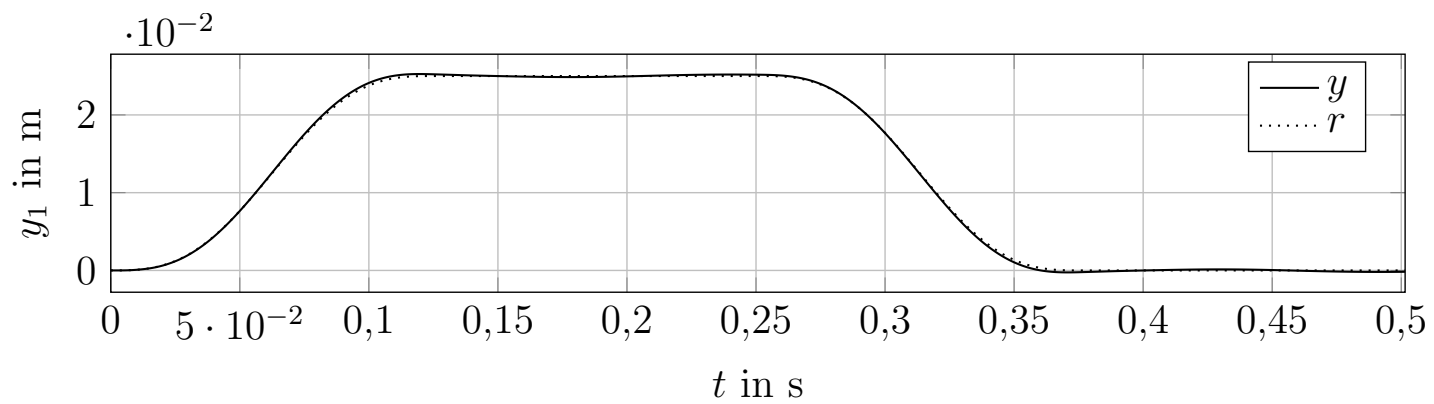

(a) Tracking of ouput 1

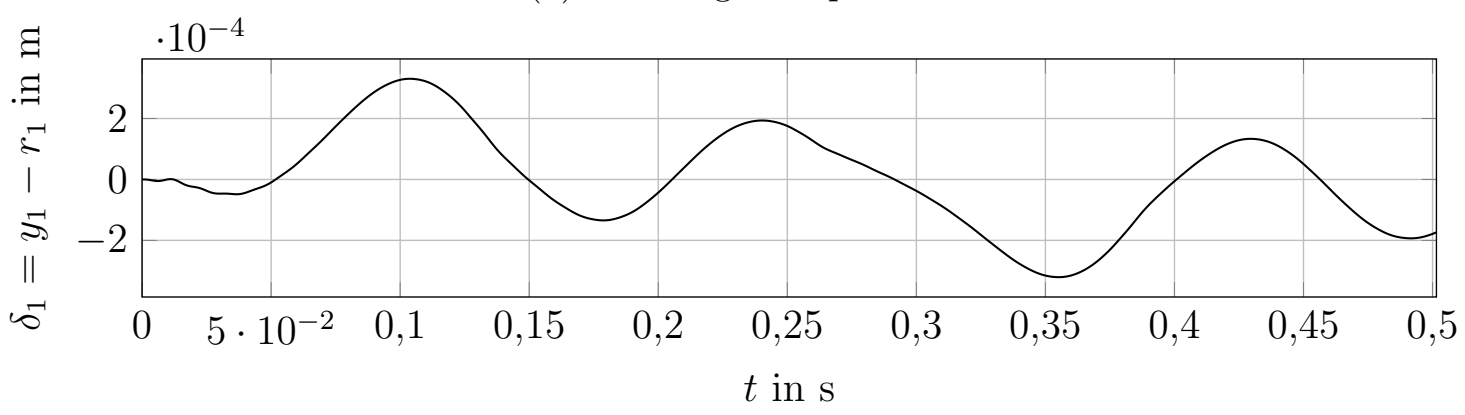

(b) Tracking error of ouput 1

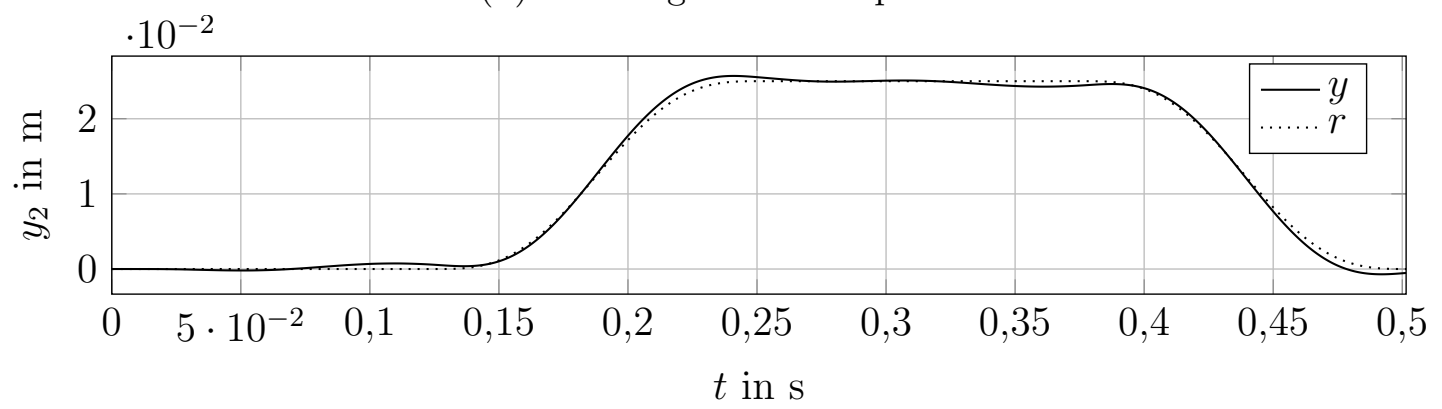

(c) Tracking of ouput 2

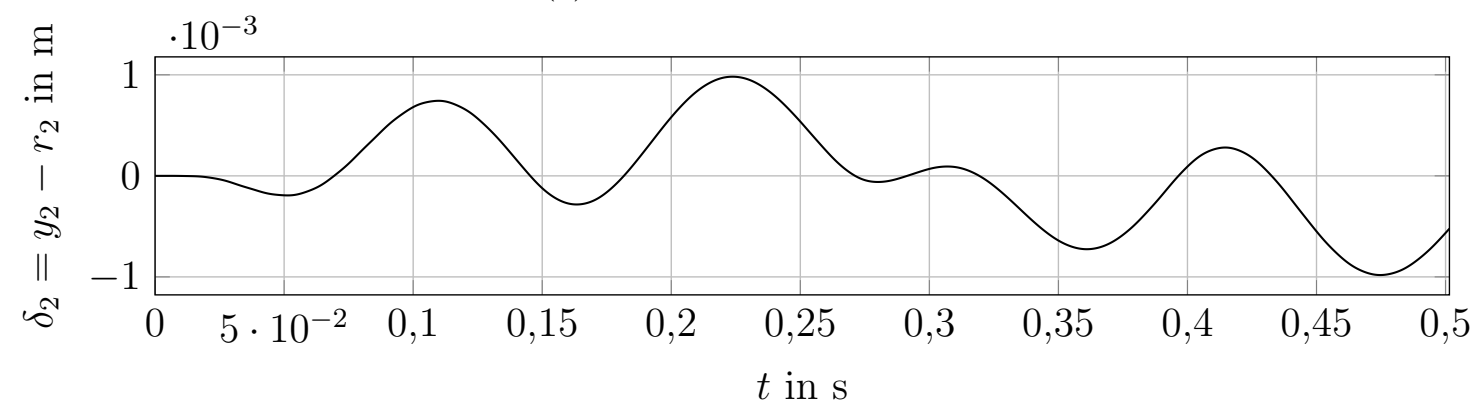

(d) Tracking error of output 2

Figure 5.6: H-Frame tracking (CSF - observer with uncertainties) 


\begin{tabular}{c|cccccc} 
& CSF & CSF - freq. opt. & CSF - unc. & IMP & IMP - freq. opt. & IMP - unc. \\
\hline$\omega_{b}$ in $\frac{\text { rad }}{\mathrm{s}}$ & 149.5312 & 298.7422 & 3.1145 & $1.4551 \times 10^{3}$ & $1.4556 \times 10^{3}$ & 3.1354 \\
$J_{e}$ & $2.4659 \times 10^{-7}$ & $2.9857 \times 10^{-8}$ & 0.0045 & $1.4852 \times 10^{-14}$ & $9.8952 \times 10^{-15}$ & 0.0045 \\
$J_{m}$ & $1.7671 \times 10^{-5}$ & $2.8290 \times 10^{-6}$ & 0.1542 & $1.0782 \times 10^{-11}$ & $1.0826 \times 10^{-11}$ & 0.1540
\end{tabular}

Table 5.2: H-Frame tracking results

function for the stability constraint $\left(\beta=10^{4}, \omega_{N}=91.9841 \frac{\mathrm{rad}}{\mathrm{s}}\right)$ and $s=s_{\max }=1$ additional advance.

The results for an uncertain plant in combination with an observer are comparable to the CSF results.

All the results can be seen in Table 5.2.

\subsection{Atomic Force Microscope}

The model for an Atomic Force Microscope (AFM) was taken from [4], where also details on the AFM can be found. As a versatile instrument, the AFM is able to image nanoscale structures, and it is of particular interest for control engineers since the imaging depends utterly on the feedback control loop. In this section, a model for motion in the $X$ direction will be considered [4]. It is given as the discrete-time transfer function model

$$
\begin{array}{r}
P_{X X}(z)=\frac{-0.0014(z-0.0061)(z-1.7824)}{(z-0.8884)(z-0.8572 \pm j 0.4032)} \\
\times \frac{(z-1.1264 \pm j 0.4627)(z-0.8762 \pm j 0.3766)}{(z-0.8717 \pm j 0.2742)(z-0.9716 \pm j 0.2022)}
\end{array}
$$

which uses a sampling rate of $f=20.833 \mathrm{kHz}$, so the sampling time is $T=\frac{1}{f}=$ $0.048 \mathrm{~ms}$.

In order to use the rules given in [27] for the design of the feedback tracking system, the poles of the discrete-time model are first mapped back to the continuous-time domain, using $s_{i}=\frac{1}{T} \ln \left(\lambda_{i}\right)$. The result is

$$
\begin{aligned}
& s_{1,2}=-1.1281 \times 10^{3} \pm j 9.1595 \times 10^{3} \quad s_{5,6}=-1.5858 \times 10^{2} \pm j 4.2745 \times 10^{3} \\
& s_{3,4}=-1.8778 \times 10^{3} \pm j 6.3490 \times 10^{3} \quad s_{7}=-2.4652 \times 10^{3} .
\end{aligned}
$$


(a) From input 1 to output 1
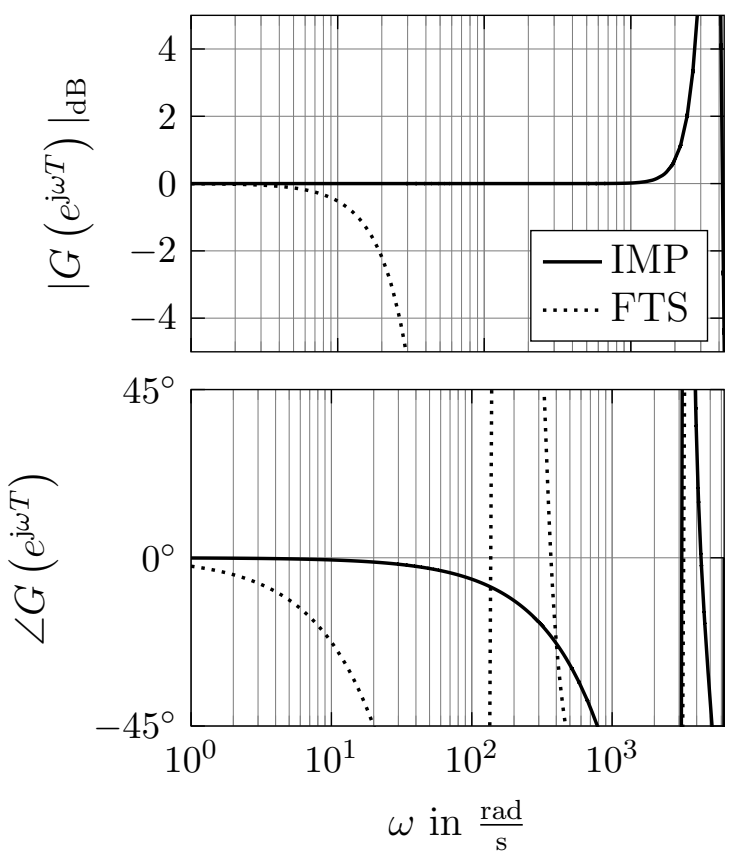

(c) From input 1 to output 2
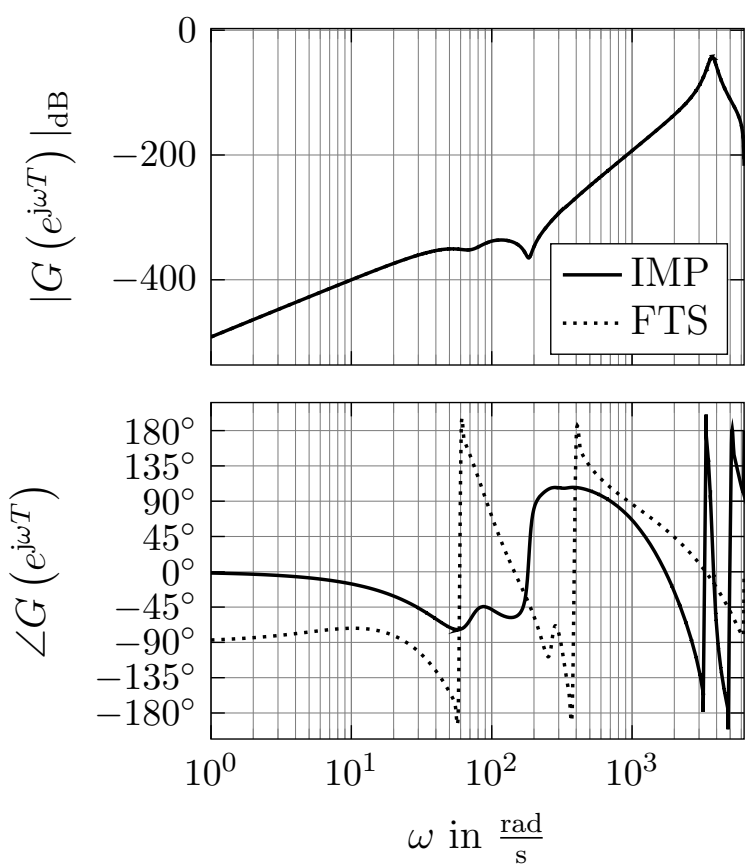

(b) From input 2 to output 1
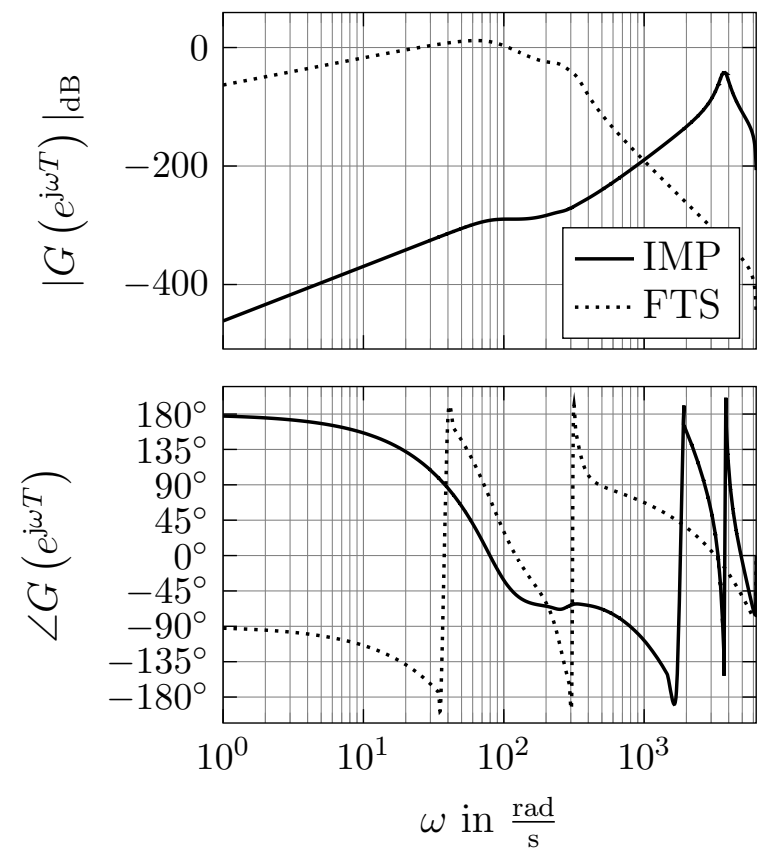

(d) From input 2 to output 2
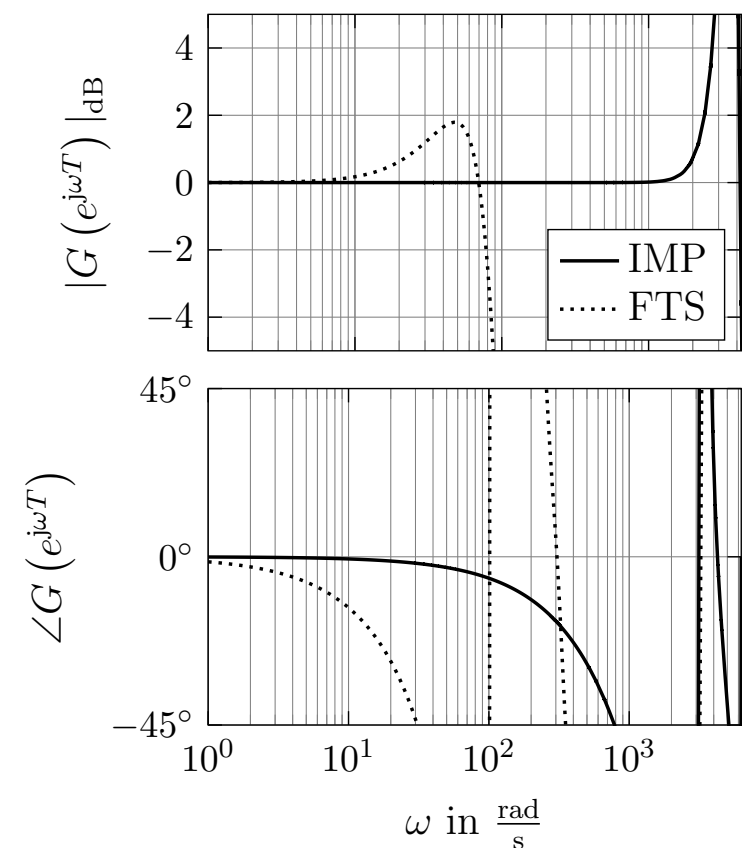

Figure 5.7: Frequency response of the H-Frame precision tracking system (IMP) 


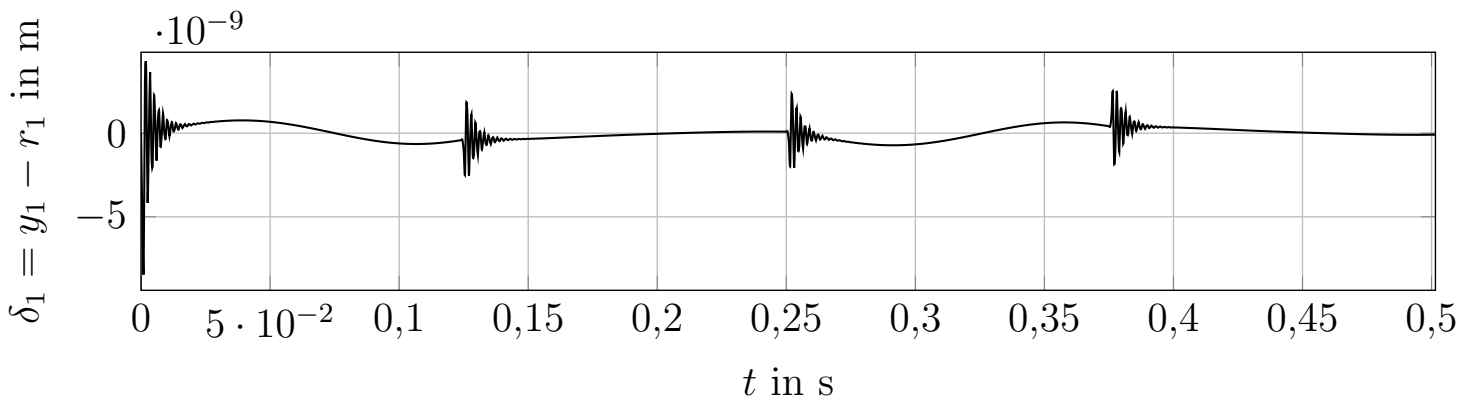

(a) Tracking error of ouput 1

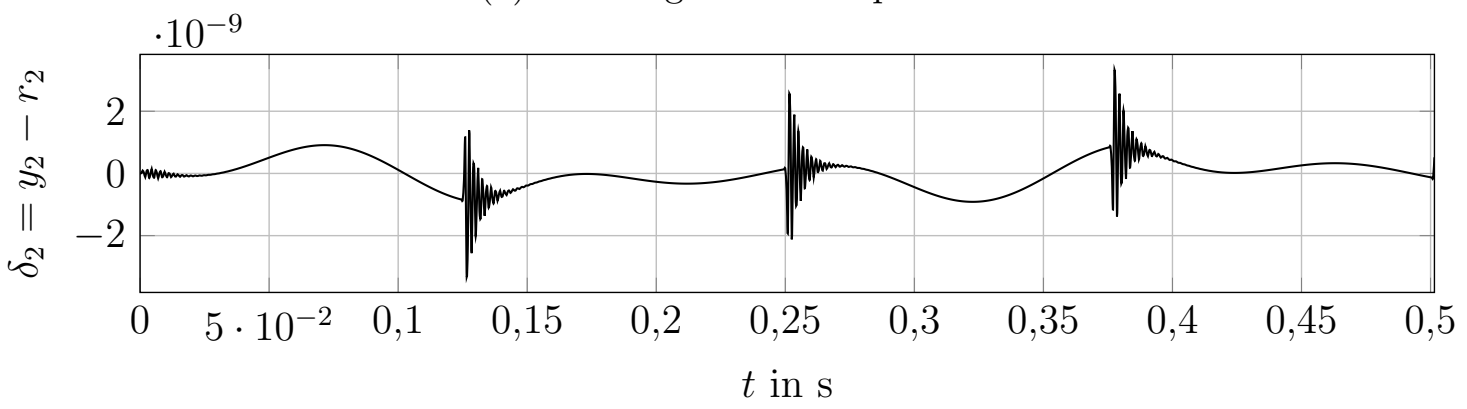

(b) Tracking error of output 2

Figure 5.8: H-Frame tracking (IMP) 
Together with integral additional dynamics, the design model (3.8) is a 8th-order system, with an additional eigenvalues in 0.

For this application, the desired settling time was set to $T_{S}=2.5 \mathrm{~ms}$. As closed-loop pole locations in the continuous-time domain, we keep the imaginary part of every pole and slide the real parts over to $\operatorname{Re}\left\{s_{i}\right\}=-2000, i=1, \ldots, 8$. For integral additional dynamics, the eigenvalue in 0 is moved to $s_{8}=-2050$, so that the closed-loop system has only distinct eigenvalues. Since the model for the $X$ motion is a SISO system (i.e. the algorithm in [27] returns the same robustness bounds as any other pole placement algorithm), Matlab's place command was used to calculate $\mathbf{K}_{d}$. After the feedback design, the digital feedback tracking system has poles at

$$
\begin{array}{ll}
\lambda_{1,2}=0.8221 \pm j 0.3867 & \lambda_{7}=0.9063 \\
\lambda_{3,4}=0.8666 \pm j 0.2726 & \lambda_{8}=0.9085 \\
\lambda_{5,6}=0.8894 \pm j 0.1851 &
\end{array}
$$

and zeros at

$$
\begin{aligned}
\eta_{1} & =0.0061 & \eta_{4} & =1.7824 \\
\eta_{2,3} & =0.8762 \pm j 0.3766 & \eta_{5,6} & =1.1264 \pm j 0.4627 .
\end{aligned}
$$

The zeros of the feedback tracking system are the same as the zeros of the discretetime plant. The resulting robustness bounds are $\delta_{1}=1$ and $\delta_{2}=0.8443$.

The reference trajectory is a triangular wave, whose fundamental frequency is $f_{0}=100 \mathrm{~Hz}$ and ranges from $-9 \mu \mathrm{m}$ to $9 \mu \mathrm{m}$ (because the microscope shall move in a "back-and-forth motion" [4]). For the simulation, the Fourier series of this signal was formed and the first $N$ harmonics were included into the reference trajectory. 
The result for the Fourier series is

$$
\begin{aligned}
b_{k} & =\frac{3.6 \times 10^{-5}}{k^{2} \pi^{2}}\left(\sin \left(k \frac{\pi}{2}\right)-\sin \left(k \frac{3 \pi}{2}\right)\right) \\
r(t) & =\sum_{k=1}^{\infty} b_{k} \sin (\underbrace{2 \pi f_{0} k}_{=\omega_{k}} \cdot t) .
\end{aligned}
$$

For the simulations in the following sections, the maximum frequency included in the reference trajectory $r(t)$ was $f_{\max }=1900 \mathrm{~Hz}$.

\section{Command Shaping Filter}

The CSF can invert the AFM model exactly with a delay of $r=2$ samples. Since $\left|\lambda_{\max }\right|=0.9085$, we have $R=0.9542$. It takes $s=14$ additional advances until the CSF is able to keep the eigenvalues of $\boldsymbol{\Phi}_{f}$ within the pole radius $R$. The cascade of the CSF and the closed-loop system becomes an approximate delay of $d=16$ samples. The Bode plot of the cascade can be seen in Fig. 5.9. The corresponding precision tracking bandwidth is $\omega_{b}=567.0700 \frac{\mathrm{rad}}{\mathrm{s}}$

Figure 5.10 shows the tracking performance (Fig. 5.10a) of the CSF as well as the resulting absolute tracking error $\delta$ (Fig. 5.10b). It can be seen that the tracking error reaches its highest value during the first rising edge of the reference trajectory, it is approximately $\left|\delta_{\max }\right|=1.8 \mu \mathrm{m}$. After this initial peak, the tracking error always stays within a range of $\pm 0.35 \mu \mathrm{m}$ around $0 \mathrm{~m}$ (indicated by the red dashed lines in Fig. 5.10b). The largest tracking errors occur at the maximums and minimums of the reference trajectory. The CSF reaches $J_{e}=4.3419 \times 10^{-4}$ and $J_{m}=0.9543$ as performance measures.

Again, the hybrid approach between frequency optimization and adding advances can be used to design the CSF. The best result is obtained for an unconstrained optimization with (4.56) as penalty function for the eigenvalue constraint and $\beta=1 \times 10^{4}$. Moreover, $s=11$ additional delays were used, and the maximum frequency included in the optimization was $\omega_{N}=1.6897 \times 10^{3} \frac{\mathrm{rad}}{\mathrm{s}}$. The spectral 

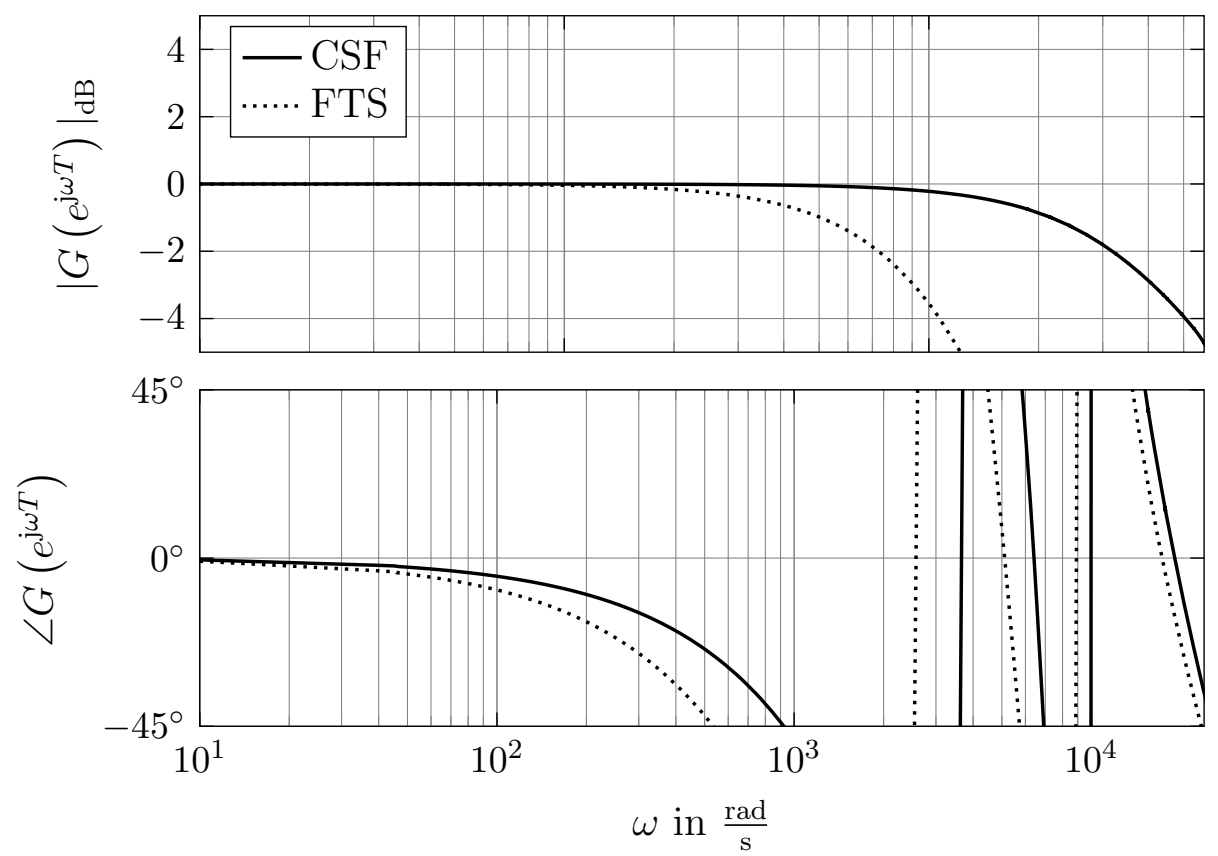

Figure 5.9: Frequency response of the AFM precision tracking system (CSF)

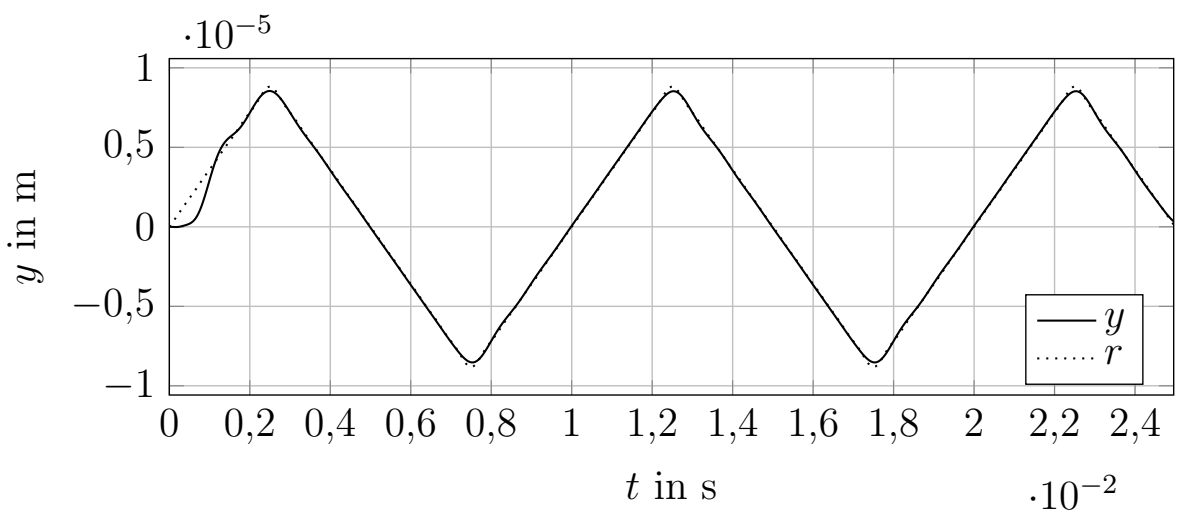

(a) Tracking result

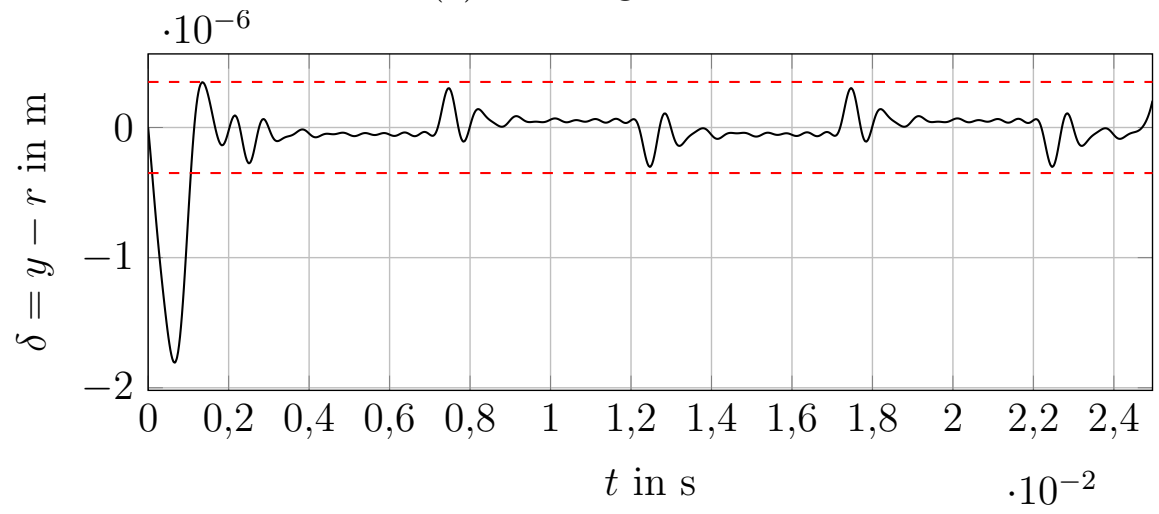

(b) Tracking error

Figure 5.10: AFM tracking (CSF) 

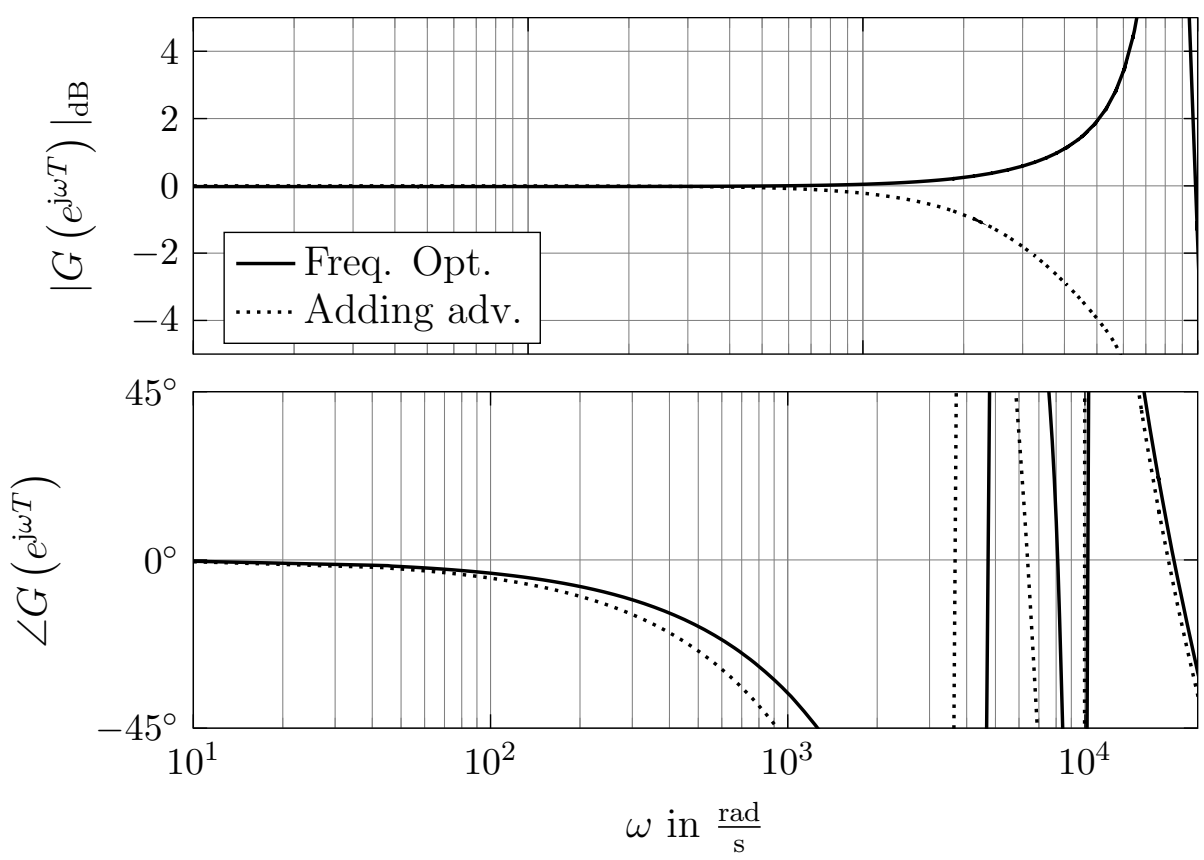

Figure 5.11: Frequency response of the AFM precision tracking system (CSF frequency optimization)

radius achieved with this approach is $\rho\left(\boldsymbol{\Phi}_{f}\right)=0.9799$.

The resulting Bode plot can be seen in Fig. 5.11, while Fig. 5.12 shows the tracking performance and error.

The precison tracking bandwidth could be greatly extended to $\omega_{b}=1.6897 \times$ $10^{3} \frac{\mathrm{rad}}{\mathrm{s}}$ (so the optimization design ensures perfect tracking over the desired frequency range), which roughly triples the previous bandwidth. The absolute value of the maximum tracking error could be improved by around $22 \%$ to $\left|\delta_{\max }\right|=1.4 \mu \mathrm{m}$. During the first maximum, however, the tracking error is larger than before $(0.77 \mu \mathrm{m}$ instead of $0.35 \mu \mathrm{m})$. After the first maximum of the reference trajectory, the tracking error stays within a range of around $\pm 0.35 \mu \mathrm{m}$ as well (as shown by the red dashed lines in Fig. 5.12b). The performance measures $J_{e}$ and $J_{m}$ do not change significantly.

Ultimately, an uncertain plant is considered, and it is assumed that the statevariables of the plant are not measurable, so that an observer has to be designed. If 


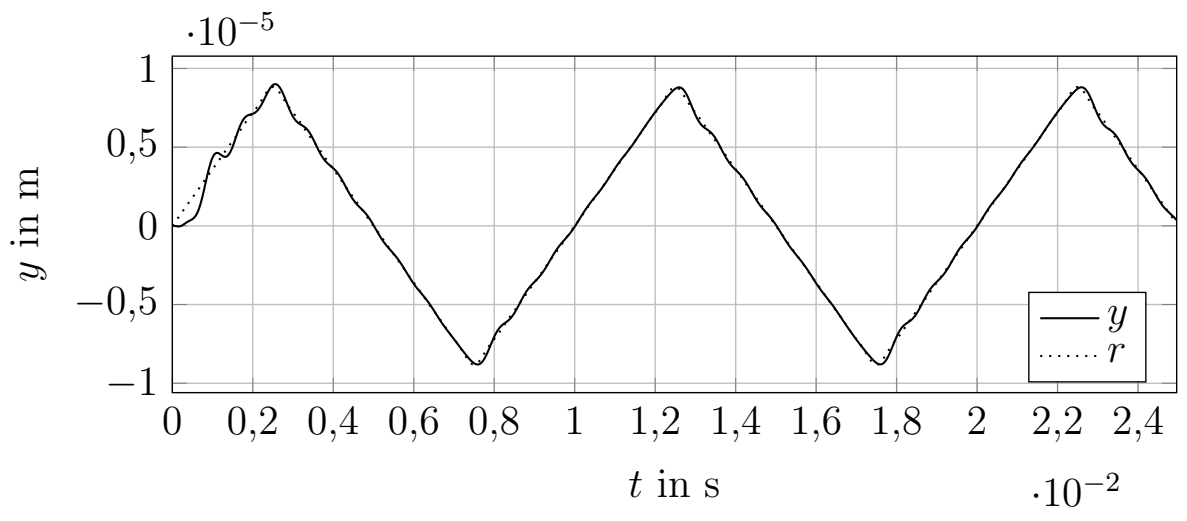

(a) Tracking performance of the AFM precision tracking system (CSF - frequency optimization)

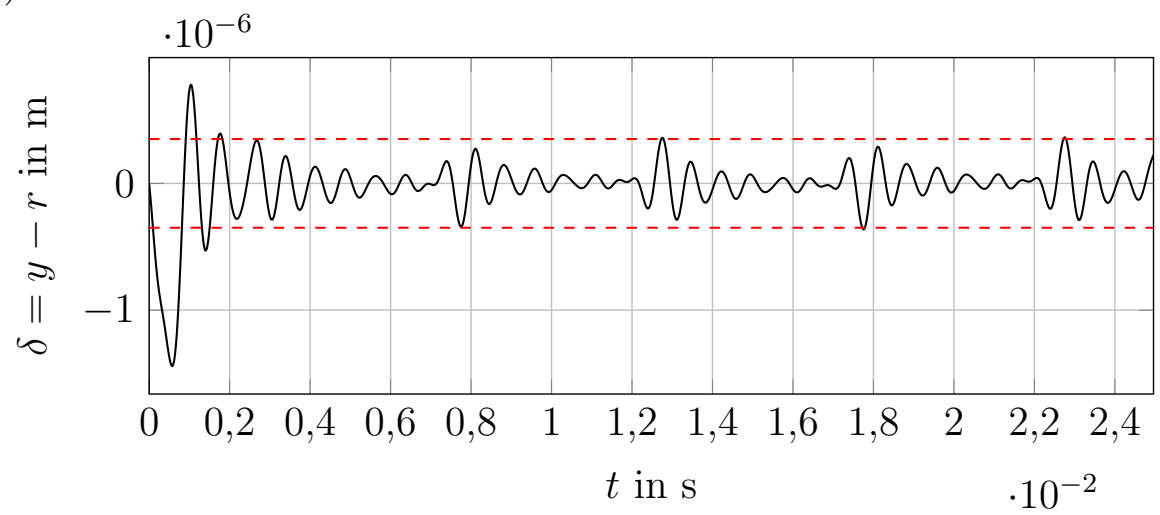

(b) Tracking error of the AFM precision tracking system (CSF - frequency optimization)

Figure 5.12: AFM tracking (CSF - frequency optimization) 
the discrete-time plant (5.26) is mapped back to continuous-time, it has six zeros, three of which are non-minimum phase. The observer gain matrix $\mathbf{L}$ is calculated according to the guidelines in [27]: the desired settling time of the observer is set to $T_{S, O}=0.5 \mathrm{~ms}$ and the minimum phase zeros are kept as poles of the tracking system and the non-minimum phase zeros are reflected on the imaginary axis and used as poles. The remaining seventh observer pole is set to the first order Bessel pole $\left(\frac{s_{1}}{T_{S, O}}\right.$, see Table 5.1) and the observer gain is calculated with place on the system $\left(\boldsymbol{\Phi}^{\mathrm{T}}, \mathbf{C}^{\mathrm{T}}\right)$. The resulting stability robustness bounds are $\delta_{1}=1$ and $\delta_{2}=0.5372$.

After that, the inverse filter is formed with the nominal plant model (5.26), but simulations are carried out with an uncertain plant model. This uncertain model is formed as follows: since $\boldsymbol{\Phi}$ denotes the nominal plant state-transition matrix, let $\widetilde{\boldsymbol{\Phi}}$ denote the state-transition matrix of the perturbed plant. Then, $\widetilde{\boldsymbol{\Phi}}$ is obtained by changing every entry of $\boldsymbol{\Phi}$ by $10 \%$, i.e. $\widetilde{\boldsymbol{\Phi}}=(1+\Delta) \boldsymbol{\Phi}$ and $\Delta=0.1$. The result is shown in Fig. 5.13. Especially during the first rising edge, the tracking system struggles to track the desired output, the output oscillates a bit around the reference input. This, however, results in a smaller absolute tracking error, as it decreases to $\left|\delta_{\max }\right|=1.5 \mu \mathrm{m}$. The main problem of the CSF in this case is that it does not reach the maximum or minimum value of the reference trajectory anymore, the absolute values at the turnaround points of the actual output are smaller than the respective values of the reference. This leads to the behaviour that the bounds of the tracking error after the initial peak grow in comparison to the previous results, the tracking error now stays within a range of $\pm 0.67 \mu \mathrm{m}$ (as indicated by the red dashed lines in Fig. 5.13b), which almost doubles the range of the initial CSF result.

The precision tracking bandwidth drops down to $\omega_{b}=190.8842 \frac{\mathrm{rad}}{\mathrm{s}}$, the performance measures are $J_{e}=5.2750 \times 10^{-4}$ and $J_{m}=0.9249$. As for the H-Frame 


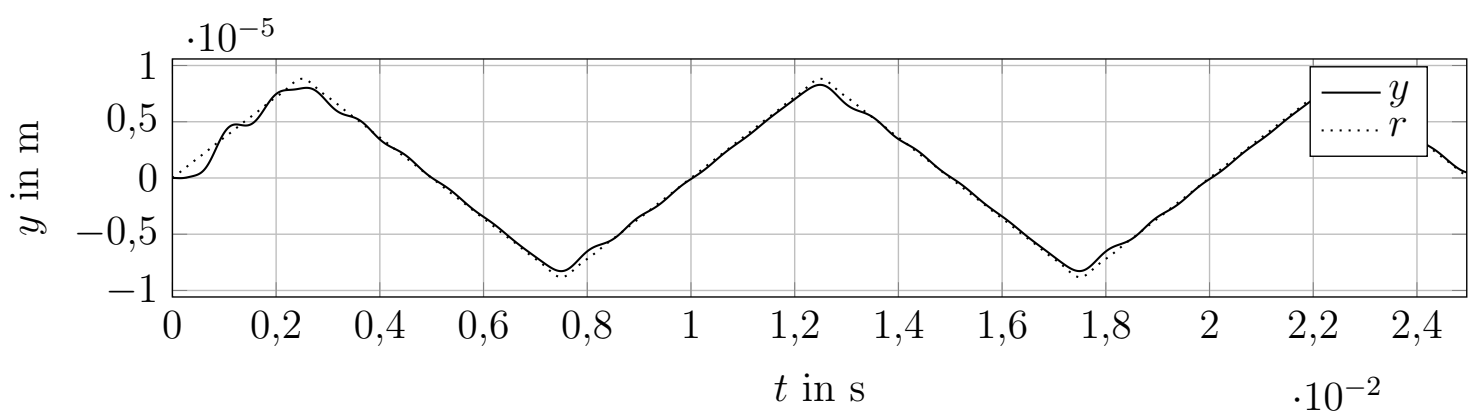

(a) Tracking performance of the AFM precision tracking system

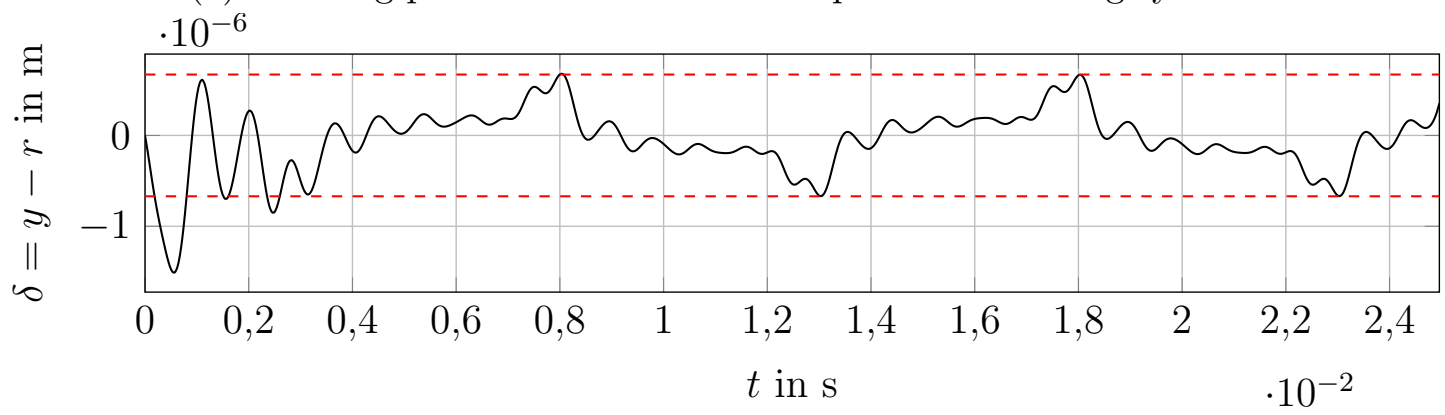

(b) Tracking error of the AFM precision tracking system

Figure 5.13: AFM tracking (CSF - uncertain plant with observer) 
system in Section 5.1, it can be concluded that acceptable tracking performances are achieved in the presence of plant uncertainties and observer state estimations.

\section{Inverse Modified Plant}

In the case of the AFM, it was examined whether the IMP delivers more satisfying results if not only integral additional dynamics are used, but the dynamics of the reference trajectory are included as additional dynamics. It can be seen in (5.30) that the reference trajectory consists of a sum of sine waves with frequency $\omega_{k}$. For the following simulations, the first harmonic $\omega_{1}$ of the reference was included in the additional dynamics, i.e. the matrix $\boldsymbol{\Phi}_{f}$ is ought to have the two (discrete-time) eigenvalues $\lambda_{1}=e^{j \omega_{1} T}$ and $\lambda_{2}=e^{-j \omega_{1} T}$. In order to guarantee that the design model (3.8) is controllable, the additional dynamics are given in controllable canonical form:

$$
\begin{array}{ll}
\boldsymbol{\Phi}_{a} & =\left[\begin{array}{cc}
0 & 1 \\
-1 & \left(e^{j \omega_{1} T}+e^{-j \omega_{1} T}\right)
\end{array}\right] \\
\boldsymbol{\Gamma}_{a} & =\left[\begin{array}{l}
0 \\
1
\end{array}\right] .
\end{array}
$$

This design did improve the overall performance of the IMP precision tracking system, especially the precision tracking bandwidth.

Therefore, the design model is a 9th-order system, which is designed in the same way as before (i.e. the real parts of the eigenvalues are moved to -2000). After the feedback tracking system design, the modified plant has the following (digital) poles

$$
\begin{array}{ll}
\lambda_{1,2}=0.9523 \pm j 0.2426 & \lambda_{5,6}=0.8243 \pm 0.3093 \\
\lambda_{3,4}=0.8167 \pm j 0.3784 & \lambda_{7}=0.6950
\end{array}
$$

and zeros

$$
\begin{aligned}
\eta_{1} & =0.0061 & \eta_{4} & =1.7824 \\
\eta_{2,3} & =0.8762 \pm j 0.3766 & \eta_{5,6} & =1.1264 \pm j 0.4627
\end{aligned}
$$



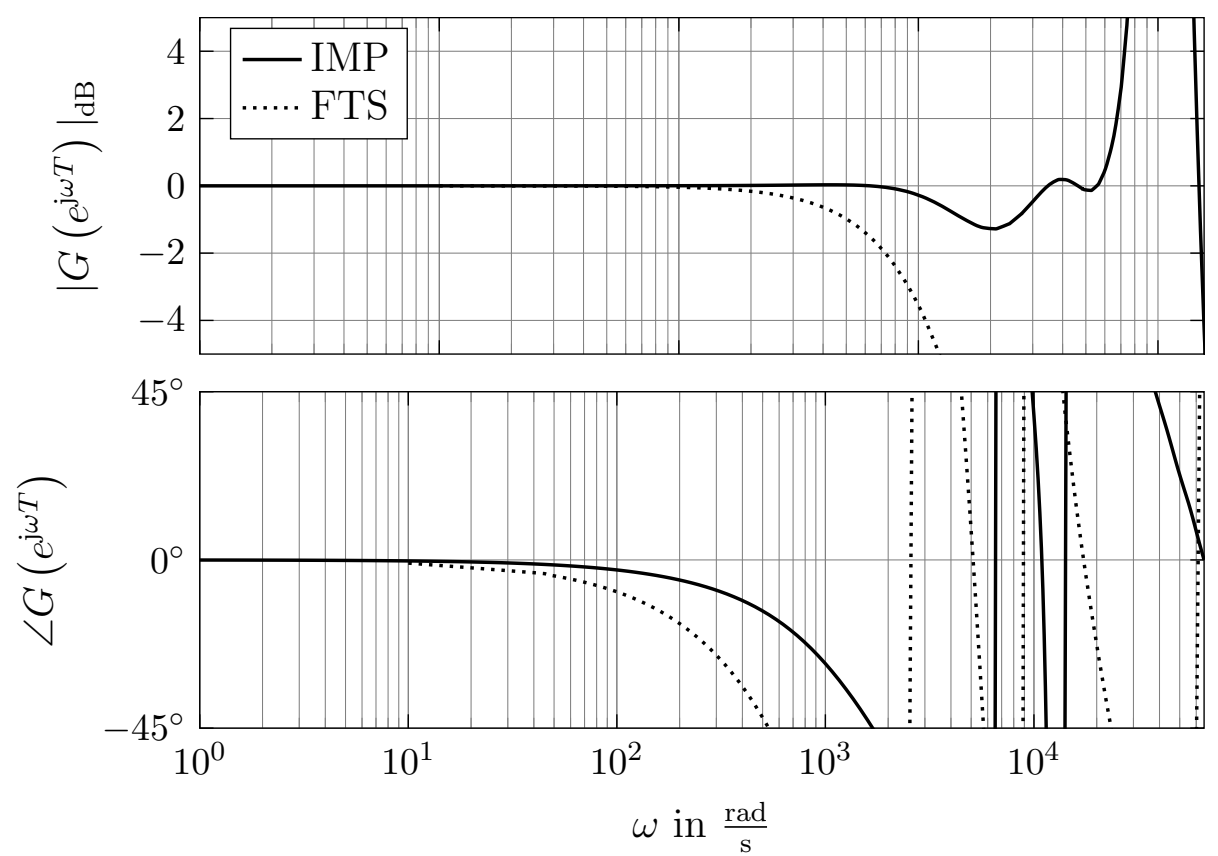

Figure 5.14: Frequency response of the AFM precision tracking system (IMP)

which coincides with the plant's zeros.

The IMP can invert the modified plant exactly with a delay of $r=1$ sample. Additional $s=9$ advances are needed to keep all the eigenvalues of the filter within $R=0.9914$. The cascade of the IMP and the modified plant is an approximate delay of $d=10$ samples. The Bode plot is shown in Fig. 5.14. The precision tracking bandwidth of $\omega_{b}=909.6986 \frac{\mathrm{rad}}{\mathrm{s}}$ is larger than for the first presented CSF result, but smaller than for the hybrid CSF approach.

In Fig. 5.15 the tracking performance (Fig. 5.15a) and error (Fig. 5.15b) can be seen. Similar to the tracking performance of the CSF, the tracking error has a peak during the first rising edge of the reference trajectory and at the first maximum. The maximum tracking error is $\left|\delta_{\max }\right|=1.3 \mu \mathrm{m}$ for the IMP, this is an improvement of $28 \%$ in comparison to the CSF, and also a slight improvement in comparison to the hybrid CSF approach. After this initial peak, the tracking error stays in between $\pm 3.5 \mu \mathrm{m}$, just as for the CSF. While $J_{e}$ has about the same value 


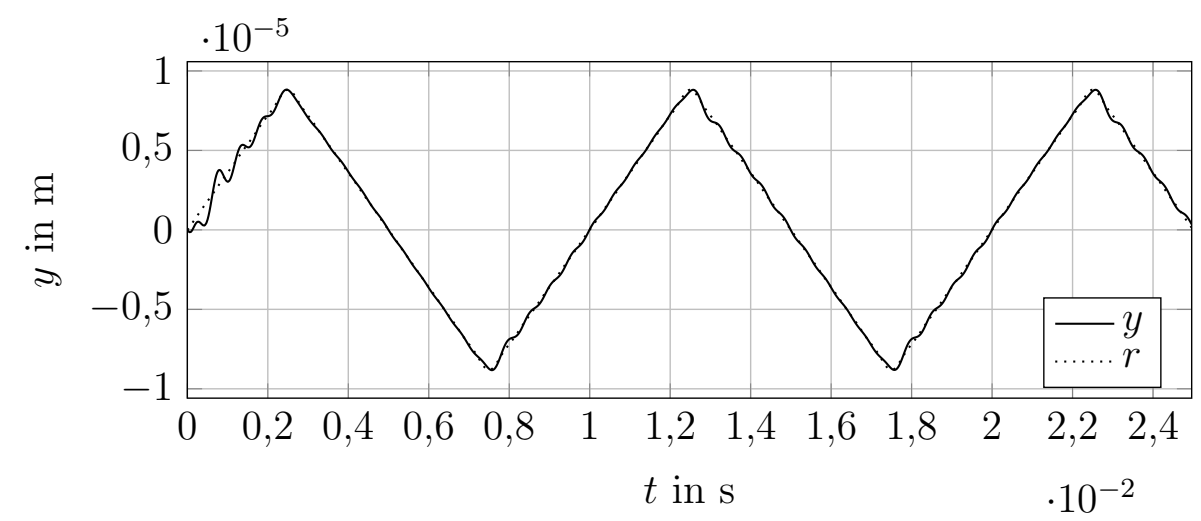

(a) Tracking performance of the AFM precision tracking system (IMP)

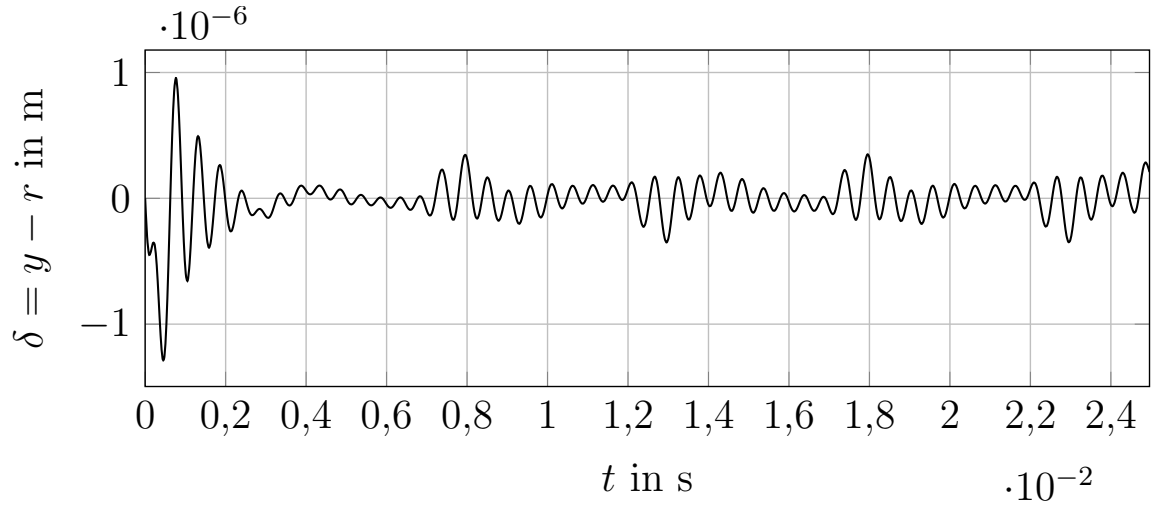

(b) Tracking error of the AFM precision tracking system (IMP)

Figure 5.15: AFM tracking (IMP)

as in the CSF case, $J_{m}$ was improved by $30.43 \%$ to $J_{m}=0.6639$ in comparison to the CSF results.

The best frequency optimization result was obtained when $s=8$ additional advances were added, and an unconstrained optimization problem was solved with (4.56) as penalty function for the stability constraint $\left(\beta=10^{4}, \omega_{N}=1.6897 \times\right.$ $\left.10^{3} \frac{\mathrm{rad}}{\mathrm{s}}\right)$

The tracking results for the AFM can be found in Table 5.3.

\section{Comparison to Source}

In this section, the tracking performance achieved with the CSF and IMP will be compared to the tracking results in [4]. In this paper, several different 


\begin{tabular}{c|cccc} 
& CSF & CSF - freq. opt. & IMP & IMP - freq. opt. \\
\hline$\omega_{b}$ in $\frac{\mathrm{rad}}{\mathrm{s}}$ & 567.0700 & $1.6897 \times 10^{3}$ & 909.6986 & $1.722 \times 10^{3}$ \\
$J_{e}$ & $5.3416 \times 10^{-4}$ & $4.0184 \times 10^{-4}$ & $2.5914 \times 10^{-4}$ & $2.9145 \times 10^{-4}$ \\
$J_{m}$ & 0.9543 & 0.9059 & 0.6639 & 0.6673
\end{tabular}

Table 5.3: AFM tracking results

approaches were considered (e.g $\mathcal{H}_{\infty}$ or $\ell_{1}$ control) to design a feedback controller $C$ and a feedforward controller $F$. Due to the fact that several approaches were considered, multiple tracking performances were presented in [4]. In general, the results presented in [4] have a larger maximal tracking error $\left|\delta_{\max }\right|$. This large tracking error is not only observed at the first turnaround point, but at every minimum and maximum of the reference trajectory.

The first approach presented is a $\mathcal{H}_{\infty}$ design. During the rising/falling edges of the reference trajectory, a good tracking performance is achieved. At the turnaround points, however, the tracking error is at almost $\left|\delta_{\max }\right|=2 \mu \mathrm{m}$. In contrast to this result, the best CSF performance improves the maximum tracking error by $30 \%$; the IMP by $35 \%$. Furthermore, the maximum tracking error for the CSF and the IMP occurred during the first rising edge, the tracking error at the later turnaround points is much smaller for both CSF and IMP: the maximum tracking error for both architectures at the later turnaround points is $0.35 \mu \mathrm{m}$, which improves the error by $82.5 \%$ in contrast to the $\mathcal{H}_{\infty}$ design.

If $C$ and $F$ are designed via an approximate model-inversion presented in $[1,23]$, the tracking error at the turnaround points could be improved to be at around $|\delta \max |=1 \mu \mathrm{m}[4]$, but it achieves a worse tracking performance away from the turnaround points. Nevertheless, CSF and IMP improve the tracking error by $65 \%$ at the turnaround points. 


\subsection{Scanning Tunneling Microscope}

Models for the $x$ - and $y$-Dynamics for a Scanning Tunneling Microscope (STM) were taken from [6]. In this application, a piezo scanner moves the STM probe across a sample surface. During this movement, the distance between the STM probe and the sample surface can be concluded from the measurement of the tunneling current, which makes it possible to form images of the sample's surface topology. The piezo scanner moves the probe parallel to the sample surface $(x-y$ axes), and the positioning of the probe is crucial, since positioning errors can lead to distortions in the images [6]. The (continuous-time) transfer function $G_{x}(s)$ of the $x$-Dynamics (from the input voltage $u_{x}$ in $\mathrm{V}$ to the piezo-position $p_{x}$ in $\AA$ ) is given by

$$
G_{x}(s)=29.28 \frac{(s-9.274 \pm j 41.659)(s+2.484 \pm j 30.434)}{(s+0.188 \pm j 31.326)(s+0.857 \pm j 24.570)(s+7.263)(s+3.198)}
$$

while

$G_{y}(s)=15.26 \frac{(s+0.7135 \pm j 16.6719)(s-44.8250 \pm j 63.1009)}{(s+0.3722 \pm j 25.6469)(s+0.2239 \pm j 31.3441)(s+8.566)(s+3.866)}$

holds for the $y$-Dynamics (from the input voltage $u_{y}$ in $\mathrm{V}$ to the piezo-position $p_{y}$ in $\AA$ ) [6]. It is important to mention that the Laplace variable $s$ is in $\frac{\mathrm{rad}}{\mathrm{ms}}$. In this thesis, the two models for $x$ - and $y$-Dynamics were combined to one single state-space system (i.e. the overall systems consists of two decoupled subsytems) with input $\boldsymbol{u}=\left[\begin{array}{ll}u_{x} & u_{y}\end{array}\right]^{\mathrm{T}}$ and output $\boldsymbol{y}=\left[\begin{array}{ll}p_{x} & p_{y}\end{array}\right]^{\mathrm{T}}$, so that the algorithms from Section 3.2 can be applied. Naturally, the state-space models contains

$$
\begin{aligned}
s_{1,2} & =-0.188 \pm j 31.326 & s_{7,8} & =-0.3722 \pm j 25.6469 \\
s_{3,4} & =-0.857 \pm j 24.570 & s_{9,10} & =-0.2239 \pm j 31.3441 \\
s_{5} & =-7.263 & s_{11} & =-8.566 \\
s_{6} & =-3.198 & s_{12} & =-3.866
\end{aligned}
$$


the poles of the models for the $x$ - and $y$-Dynamics as eigenvalues $\left(s_{1}-s_{6}\right.$ belong to the $x$-model, $s_{7}-s_{12}$ to the $y$-model).

Together with the additional dynamics, the design model is a 14th-order system. For the design of the feedback tracking system, the desired settling time is set to $T_{S}=0.5 \mathrm{~ms}$. According to the rules in [27], damping was added to the complex poles, and the remaining six real-valued poles $\left(s_{5}, s_{6}, s_{11}, s_{12}\right.$ and the two integrator poles from the additional dynamics) were moved to the 6th order Bessel poles (see Table 5.1). Based on the desired settling time, the sampling time was chosen to be $T=2 \mu \mathrm{s}$. The discrete-time modified plant has four non-minimum phase zeros, as well as the feedback tracking system. The tracking system (without inverse filter) has a precision tracking bandwidth of $\omega_{b}=0.1 \frac{\mathrm{rad}}{\mathrm{ms}}$.

The reference trajectory causes the STM probe to move in a raster pattern [6]. First, the probe is moved from the center to the top-left point of the image area. During the forward (left-right) scan, the y-position is fixed, and it is incremented while the x-position is returned back to the left. This procedure is repeated until the entire desired are is scanned. In this case, the scan rate is $s_{r}=\frac{1}{T_{r}}=250 \mathrm{~Hz}$, i.e. $T_{r}$ is the time to complete one back-and-forth motion. This is illustrated in Fig. 5.16.

\section{Command Shaping Filter}

The feedback tracking system can be exactly inverted with a delay of $r=2$ samples. Because the spectral radius is $\rho\left(\boldsymbol{\Phi}_{c}\right)=0.9985$, the pole radius is $R=$ 0.9993. The CSF needs $s=36$ additional advances to keep the eigenvalues of the inverse filter within that radius, hence the cascade of filter and feedback tracking system is an approximate delay of $d=38$ samples. The CSF achieves a precision tracking bandwidth of $\omega_{b}=1.9189 \frac{\mathrm{rad}}{\mathrm{ms}}$, which extends the bandwidth almost by a factor of 20 in comparison to the simple feedback tracking system. 


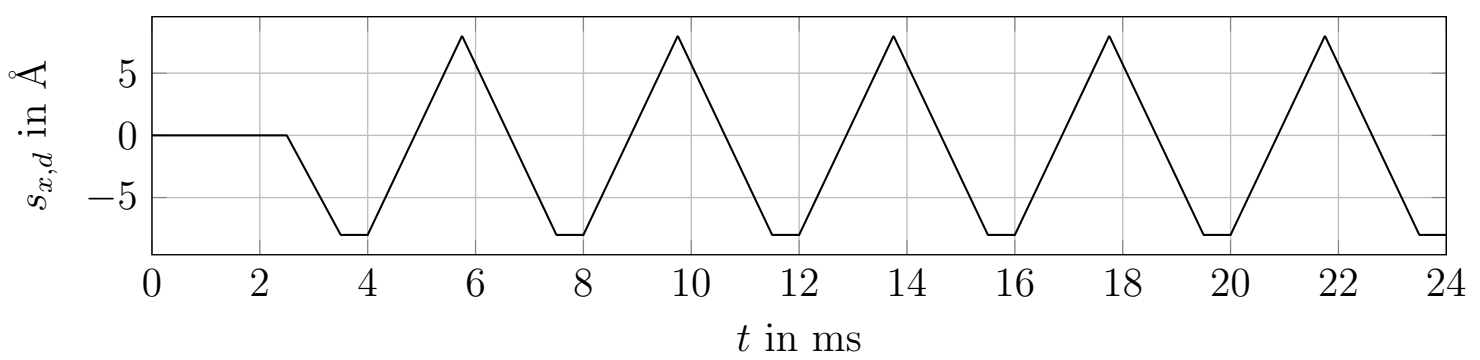

(a) Reference trajectory for the $x$-axis

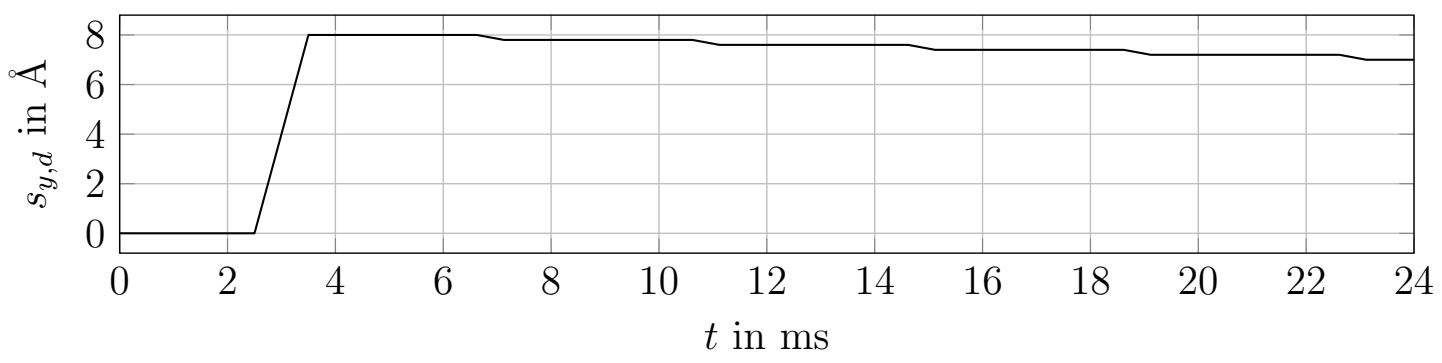

(b) Reference trajectory for the $y$-axis

Figure 5.16: STM reference trajectories

The tracking error is always smaller than $\left|\delta_{\max }\right|=0.26 \AA$, while the maximum tracking error in the simulation results presented in [6] is at approximately $\left|\delta_{\max }\right|=$ $0.6 \AA$, i.e. the tracking error shrank by $57 \%$ in comparison to the result in [6]. It can be stated that the tracking error for the first output, $\delta_{1}$, oscillates with a relatively high frequency around $0 \AA$. This also translates to the input voltage $u_{x}$, so it would be necessary to evaluate on the physical system if this control input was realizable.

The performance measures obtained by the CSF are $J_{e}=0.0162$ and $J_{m}=$ 0.0357 .

\section{Inverse Modified Plant}

The modified plant needs $r=1$ delay to be perfectly inverted. Its spectral radius is $\rho\left(\Phi_{c}\right)=0.9986$, consequently the pole radius becomes $R=0.9993$ and for this $R$, the IMP requires $s=27$ additional delays. The corresponding precision 


\begin{tabular}{c|cc} 
& CSF & IMP \\
\hline$\omega_{b}$ in $\frac{\mathrm{rad}}{\mathrm{ms}}$ & 1.9189 & 2.6906 \\
$J_{e}$ & 0.0162 & 0.0039 \\
$J_{m}$ & 0.0357 & 0.0366
\end{tabular}

Table 5.4: STM tracking results

tracking bandwidth is $\omega_{b}=2.6906 \frac{\mathrm{rad}}{\mathrm{ms}}$, thus the bandwidth was increased by $40 \%$ in comparison to the CSF result. However, the maximum absolute tracking error is a bit higher than for CSF tracking, namely $\left|\delta_{\max }\right|=0.34 \AA$. The performance measures for the IMP are $J_{e}=0.0039$ and $J_{m}=0.0366$. Especially $J_{e}$ is much smaller than for the CSF case, we have $\frac{J_{e, \mathrm{CSF}}}{J_{e, \mathrm{IMP}}} \approx 4$.

A summary of the obtained results is given in Table 5.4.

\subsection{Bell 205 Helicopter}

In $[18,19]$, the linearized model of a Bell 205 helicopter is considered. The model is a non-minimum-phase near non-hyperbolic system (i.e. the non-minimum phase zeros are close to the imaginary axis), and the model represents the helicopter at a nominal $5^{\circ}$ pitch attitude, with mid-range weight, a mid-position center of gravity, and operating in-ground effect at near sea level [18]. 
The $(\mathbf{A}, \mathbf{B}, \mathbf{C})$ continous-time model is given as [19]:

$$
\begin{aligned}
& \mathbf{A}=\left[\begin{array}{cccccccc}
0 & 0.03 & 0.18 & -0.01 & -0.42 & 0.08 & -9.81 & 0 \\
-0.1 & -0.39 & 0.09 & -0.1 & -0.72 & 0.68 & 0 & 0 \\
0.01 & -0.01 & -0.19 & 0 & 0.23 & 0.04 & 0 & 0 \\
0.02 & 0 & -0.41 & -0.05 & -0.27 & 0.27 & 0 & 9.81 \\
0.03 & -0.02 & -0.88 & -0.04 & -0.57 & 0.14 & 0 & 0 \\
-0.01 & -0.02 & -0.06 & 0.07 & -0.32 & -0.71 & 0 & 0 \\
0 & 0 & 1 & 0 & 0 & 0 & 0 & 0 \\
0 & 0 & 0 & 0 & 1 & 0 & 0 & 0
\end{array}\right] \\
& \mathbf{B}=\left[\begin{array}{cccc}
0.08 & 0.13 & 0 & 0 \\
-1.17 & 0.04 & 0 & 0.01 \\
0 & -0.07 & 0 & 0.01 \\
-0.04 & 0 & 0.11 & 0.19 \\
-0.04 & 0 & 0.22 & 0.17 \\
0.17 & 0 & 0.03 & -0.47 \\
0 & 0 & 0 & 0 \\
0 & 0 & 0 & 0
\end{array}\right] \\
& \mathbf{C}=\left[\begin{array}{llllllll}
1 & 0 & 0 & 0 & 0 & 0 & 0 & 0 \\
0 & 1 & 0 & 0 & 0 & 0 & 0 & 0 \\
0 & 0 & 0 & 1 & 0 & 0 & 0 & 0 \\
0 & 0 & 0 & 0 & 0 & 1 & 0 & 0
\end{array}\right]
\end{aligned}
$$

The input vector is $\boldsymbol{u}=\left[\begin{array}{llll}\delta_{C} & \delta_{B} & \delta_{A} & \delta_{P}\end{array}\right]^{\mathrm{T}}$ where $\delta_{C}$ is collective, $\delta_{B}$ longitudinal, $\delta_{A}$ lateral cyclic and $\delta_{P}$ tail rotor collective, the output vector is $\boldsymbol{y}=\left[\begin{array}{llll}U & W & V & R\end{array}\right]^{\mathrm{T}}$ where $U$ is forward, $W$ vertical, $V$ lateral velocity and $R$ yaw rate. The plant has the following poles

$$
\begin{array}{ll}
s_{1,2}=0.1313 \pm j 0.5839 & s_{7}=-1.0006 \\
s_{3,4}=0.1590 \pm j 0.4181 & s_{8}=-0.4893 \\
s_{5,6}=-0.5004 \pm j 0.3499 &
\end{array}
$$

and the design model (3.8) additionally has four poles in 0, from the integral additional dynamics. A dlqr design was used to calculate the feedback gain $\mathbf{K}_{d}$ which stabilizes the plant and leads to the following (digital) poles of the feedback 


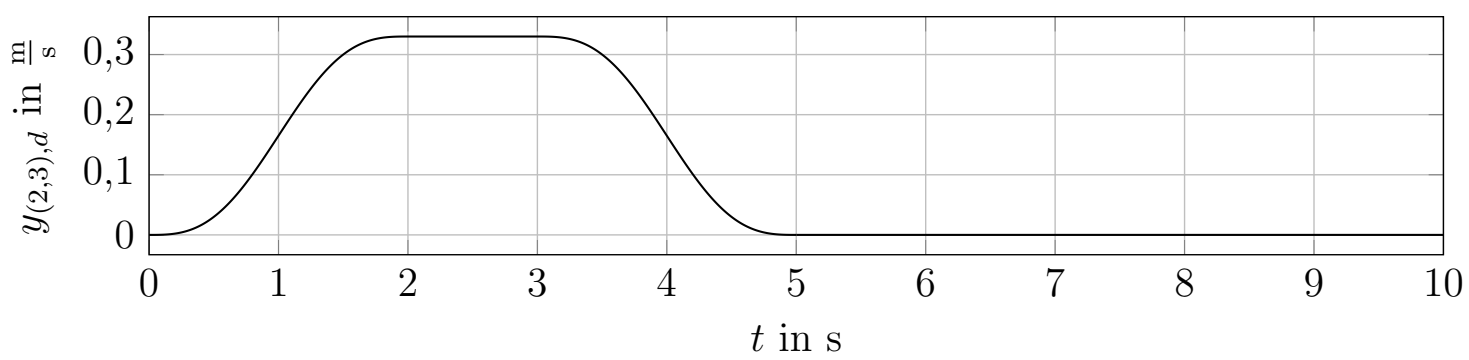

Figure 5.17: Helicopter reference trajectory for $y_{2}$ and $y_{3}$

tracking system, with a sampling time $T=0.004 \mathrm{~s}$ :

$$
\begin{array}{lrl}
\lambda_{1,2}=0.9512 \pm j 0.0464 & \lambda_{7,8}=0.9805 \pm j 0.0132 \\
\lambda_{3,4}=0.9683 \pm j 0.0304 & \lambda_{9,10}=0.9993 \pm j 0.0132 \\
\lambda_{5,6}=0.9823 \pm j 0.0153 & \lambda_{11,12}=0.9970 \pm j 0.0150
\end{array}
$$

while

$$
\begin{aligned}
& \eta_{1,2}=1.0000 \pm j 0.0172 \\
& \eta_{3,4}=0.9998 \pm j 0.0091
\end{aligned}
$$

holds for the zeros. The regulator achieves $\delta_{1}=0.5040$ and $\delta_{2}=0.9533$ as stability robustness bounds.

The inverse filter shall help to keep the forward velocity and the yaw rate $\left(y_{1}\right.$ and $\left.y_{4}\right)$ at zero and to track the trajectory shown in Fig. 5.17 with the vertical and lateral velocities $\left(y_{2}\right.$ and $\left.y_{3}\right)$.

\section{Command Shaping Filter}

The feedback tracking system possesses a spectral radius of $\rho\left(\mathbf{\Phi}_{c}\right)=0.9993$, the pole radius is $R=0.9997$. The system can be exactly inverted with a delay of $r=2$ samples, but the CSF algorithm needs $s=121$ additional advances to maintain the desired pole radius. With the chosen sampling time $T$, this corresponds to a preview time of $T_{\text {preview }}=0.484 \mathrm{~s}$. Apparently, this preview time is too large for the approximation made in Section 3.2 to be valid, since the tracking results with 
this pole radius were far away from being satisfactory. For that reason, we ask the inversion algorithm to "simply" stabilize the CSF without further pole radius demands, i.e. $R$ is set to $R=1$. Now, $s=32$ additional advances are needed until the algorithm terminates. The CSF is pretty successful in keeping $y_{1}$ and $y_{4}$ at zero. The absolute value of $y_{4}[k]$ is never larger than $4.4 \times 10^{-3} \frac{\mathrm{rad}}{\mathrm{s}}$, and $y_{1}[k]$ is upper bounded by $8.4 \times 10^{-4} \frac{\mathrm{m}}{\mathrm{s}}$. Moreover, the tracking performance of $y_{3}[k]$ is pretty well, too: the tracking error is always stays within $\left|\delta_{3, \max }\right|=4.4 \times 10^{-3} \frac{\mathrm{m}}{\mathrm{s}}$. The second output, however, does not perform as good as the third one. The maximum absolute value of the tracking error is $\left|\delta_{2, \max }\right|=16.2 \times 10^{-3} \frac{\mathrm{m}}{\mathrm{s}}$, so four times larger than for $y_{3}$.

The CSF has a precision tracking bandwidth of $\omega_{b}=0.2081 \frac{\mathrm{rad}}{\mathrm{s}}$, and the limiting transfer function is $g_{22}\left(e^{j \omega T}\right)$. The frequencies that are included in the discrete Fourier transform (DFT) if $N=2502$ values $r_{2}[k]$ are considered (from $t_{0}=0 \mathrm{~s}$ to $t_{\mathrm{end}}=10 \mathrm{~s}$ with $\left.\Delta t=t_{i}-t_{i-1}=T\right)$ are $\omega_{k}=\frac{2 \pi \cdot k}{N \cdot T}, k=0, \ldots, N-1$. It follows that the closest (nonzero) frequency included in the DFT to $\omega_{b}$ is $\omega_{1}=$ $0.62781 \frac{\mathrm{rad}}{\mathrm{s}}$, but only $83.45 \%$ of the energy of the reference trajectory shown in Fig. 5.17 is included up to $\omega_{1}$, which explains the relatively poor tracking performance of the second output.

For the CSF, the performance measures are $J_{e}=0.0143$ and $J_{m}=0.0847$.

A hybrid approach between frequency optimization and adding advances has been carried out for the helicopter as well. It proved to be most successful to solve an unconstrained minimization problem with (4.56) as penalty function for the stability constraint and (4.50) as cost function for the off-diagonal elements, where $\alpha=10$ and $\beta=10^{4}$ were used as weights. The best result was obtained when $s=4$ additional advances were added to the inversion control law, and then $\mathbf{F}$ was designed to minimize the frequency error between the tracking system and the 
ideal tracking behaviour, so that the cascade of inverse filter and feedback tracking system is an approximate delay of $d=6$ samples. After the optimization (with $\left.\omega_{N}=17.829 \frac{\mathrm{rad}}{\mathrm{s}}\right)$, the CSF has a spectral radius of $\boldsymbol{\Phi}_{f}=0.9998$.

With this approach, the precision tracking bandwidth is $\omega_{b}=0.6889 \frac{\mathrm{rad}}{\mathrm{s}}$, which is more than three times higher than the prior precision tracking bandwidth. Now, the closest frequency to $\omega_{b}$ is $\omega=1.2556 \frac{\mathrm{rad}}{\mathrm{s}}$, and $97.86 \%$ of the reference trajectory's energy is included within this frequency range.

The absolute tracking error of the second output could be decreased significantly by this approach, however, the third output now shows the worst tracking performance. The tracking error is, in this case, upper bounded by $\left|\delta_{\max }\right|=5.8 \times 10^{-3} \frac{\mathrm{m}}{\mathrm{s}}$, which is an improvement of $31 \%$ in comparison to the worst previous result. Additionally, $y_{1}$ could be kept closer to zero, the absolute value of $y_{1}$ is now upper bounded by $1.8 \times 10^{-5} \frac{\mathrm{m}}{\mathrm{s}}$, the bound for $y_{4}$ shrank slightly to $3 \times 10^{-4} \frac{\mathrm{m}}{\mathrm{s}}$

Both performance measures could be reduced significantly: $J_{e}$ now reads as $J_{e}=7.565 \times 10^{-4}$, i.e. we have a factor of almost 20 between this result and the previous one. The other performance measure, $J_{m}$, was decreased to $J_{m}=0.0016$, meaning that the factor between this result and the previous one is even higher, namely almost 55 .

\section{Inverse Modified Plant}

The spectral radius of the modified plant is $\rho\left(\boldsymbol{\Phi}_{c}\right)=0.9993$, so the pole radius would theoretically be $R=0.9996$, but similar to the CSF, the IMP would need a very large amount of additional delays $(s=238)$ to maintain this pole radius and the obtained tracking performance is pretty bad. Therefore, the pole radius is again set to $R=1$. Because the modified plant can be inverted exactly with a delay of $r=1$ sample, and the algorithm needs $s=51$ additional advances to 


\begin{tabular}{c|cccc} 
& CSF & CSF - freq. opt. & IMP & IMP - freq. opt. \\
\hline$\omega_{b}$ in $\frac{\mathrm{rad}}{\mathrm{s}}$ & 0.2081 & 0.6889 & 0.4417 & 1.3847 \\
$J_{e}$ & 0.0143 & $7.565 \times 10^{-4}$ & 0.2696 & 0.002 \\
$J_{m}$ & 0.0847 & 0.0016 & 1.1542 & 0.114
\end{tabular}

Table 5.5: Bell 205 Helicopter tracking results

stabilize the IMP, the whole tracking system is an approximate delay of $d=52$ samples.

In comparison to the CSF, slightly worse results are obtained. The deviation from $0 \frac{\mathrm{m}}{\mathrm{s}}$ for outputs one and four are comparable to the error in $y_{1}$ for the CSF, they are $\left|\delta_{1, \max }\right|=3.6 \times 10^{-3} \frac{\mathrm{m}}{\mathrm{s}}$ and $\left|\delta_{4, \max }\right|=3.9 \times 10^{-3} \frac{\mathrm{m}}{\mathrm{s}}$. In the IMP case, the limiting factor (bandwidth-wise) is the third output, the IMP reaches a precision tracking bandwidth of $\omega_{b}=0.4417 \frac{\mathrm{rad}}{\mathrm{s}}$. The maximum tracking errors for $y_{2}$ and $y_{3}$ are $\left|\delta_{2, \max }\right|=8 \times 10^{-3} \frac{\mathrm{m}}{\mathrm{s}}$ and $\left|\delta_{3, \max }\right|=35.5 \times 10^{-3} \frac{\mathrm{m}}{\mathrm{s}}$, respectively. The large value for $\left|\delta_{3, \max }\right|$ in comparison to the frequency optimization approach in the CSF case can be explained with the smaller precision tracking bandwidth. This manifests itself in the performance measures as well, as they increase to $J_{e}=0.2696$ and $J_{m}=1.1542$.

The best frequency optimization approach is obtained when $s=2$ additional advances are added, and then the optimization is carried out with (4.50) and (4.56) as cost and penalty function, respectively $\left(\alpha=10, \beta=10^{4}, \omega_{N}=17.829 \frac{\mathrm{rad}}{\mathrm{s}}\right)$. The precison tracking bandwidth becomes $\omega_{b}=1.3847 \frac{\mathrm{rad}}{\mathrm{s}}$, and $J_{e}=0.002, J_{m}=$ 0.114 follows for the performance measures. The precision tracking bandwidth in comparison to the CSF optimization result turns out to be larger, while the performance measures get slightly worse.

All the results can be found in Table 5.5. 


\section{Comparison to Source}

In [19], an adaption for the method from [15] is presented (see Section 2.1 for details). This adaption is introduced in order to be able to deal with nonhyperbolic systems. In the framework presented in [19], non-minimum phase zeros close to the imaginary axis result in a very large preactuation time. Therefore, the major idea presented in [19] is to first introduce feedback to move the nonminimum phase zeros further away from the imaginary axis, and then to apply the stable inversion for non-minimum phase system from [15]. It is a trade-off between precision tracking and required preactuation time.

The trade-off approach in [19] especially suffers from a (comparatively) poor tracking performance for the yaw rate $R$ (output $y_{4}$ ), for it has a maximum value

of $\left|\delta_{4, \max }\right| \approx 0.015 \frac{\mathrm{rad}}{\mathrm{s}}$. The maximum tracking error for the forward velocity $\left(y_{1}\right)$ is $\left|\delta_{1, \max }\right| \approx 2 \times 10^{-3} \frac{\mathrm{m}}{\mathrm{s}}$. It has to be noticed that the tracking errors are so small that they cannot be distinguished from zero if the approach from [15] is used without moving the near non-hyperbolic zeros first, so this result cannot be compared with the CSF and IMP performance. The apprehension that this results in a large preactuation time, however, proved to be justified, i.e. the practicality of this result is limited.

On the other hand, the tracking errors of the vertical and lateral velocities are not shown in detail in [19], so that a discussion and comparison of the results is not possible.

\subsection{Multilink Flexible Manipulator}

The model for a multilink flexible manipulator was taken from [43]. They are used in fields like assembling of electronic hardware, space exploration or precision welding, but they suffer from vibrating links at high operation speeds, which delays the precise positioning of the end effector [43]. Since conditioning of the 


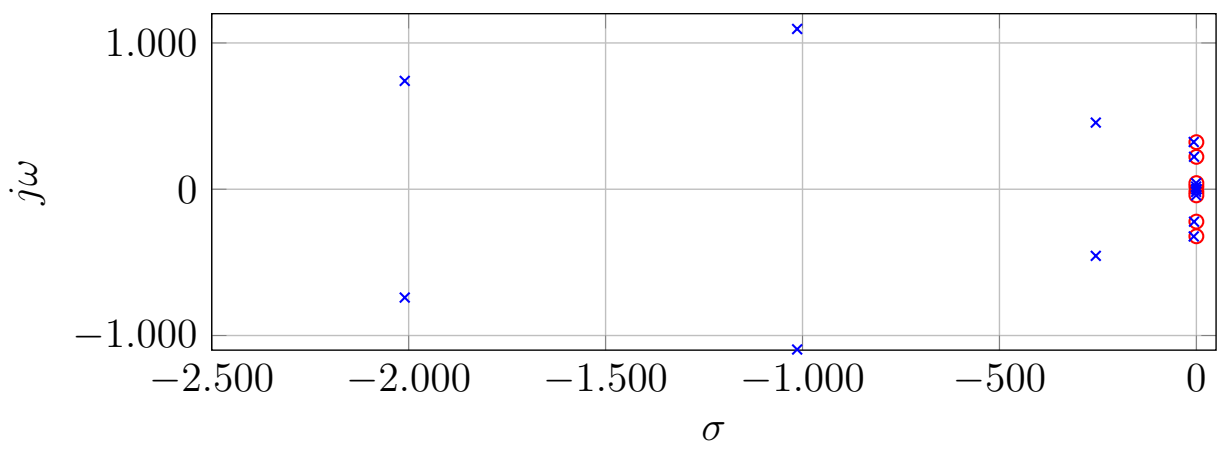

Figure 5.18: Pole zero map of the continuous-time flexible manipulator plant

manipulator excitation signal can reduce the link vibrations, the authors in [43] proposed an inverse controller to damp the vibrations.

The considered plant is a two link, 3D fexlible manipulator with three rotary joints driven by de servomotors; the model for the manipulator is a 17th-order, three input and three output, system with the joint angles as outputs. The pole zero map is shown in Fig. 5.18. The plant has eight zeros, all of them are very close to the imaginary axis. Four of the plant zeros are located in the left-half plane, the remaining four in the right half plane of the complex plane. The design model (3.8) has three additional poles in 0 (integrator additional dynamics).

For the design of the feedback tracking system, the desired settling time is set to $T_{S}=2.5 \mathrm{~s}$, which leads to a sampling time of $T=6.25 \mathrm{~ms}$. According to the rules in [27], ten plant eigenvalues were kept at their respective spot, damping was added to four of them and the remaining six were chosen as the 6th-order Bessel poles. The feedback gain was calculated with the algorithm in [27], $\delta_{1}=0.4713$ and $\delta_{2}=0.6574$ were attained as the stability robustness bounds. The resulting feedback tracking system has four non-minimum phase zeros on the unit circle.

A step with an amplitude of $20^{\circ}$ lasting for $10 \mathrm{~s}$ after which the manipulator goes back to its vertical position for ten more seconds is the reference trajectory. Since a step would cause infinite joint velocities during the rising and falling edges, 
leading to a potential mechanical breakdown of the manipulator, the reference is filtered with with the following lowpass-filter [43]:

$$
f(s)=\frac{1}{(\kappa s+1)^{n}} .
$$

The order of the filter is set to $n=2$, and with the adjustable paramter $\kappa$ the filter roll-off can be changed, which determines the speed of the response [43]. In this case, it was chosen to be $\kappa=0.2$.

\section{Command Shaping Filter}

The spectral radius for the feedback tracking system is $\rho\left(\boldsymbol{\Phi}_{c}\right)=0.9895$ so that the pole radius becomes $R=0.9948$. The exact inversion is possible with $r=2$ delays, the CSF, however, needs $s=17$ additional delays to attain the pole radius $R$, which makes the filter-based tracking system an approximate delay of $d=19$ samples.

With this pole radius, the CSF achieves a precision tracking bandwidth of $\omega_{b}=$ $3.2099 \frac{\mathrm{rad}}{\mathrm{s}}$, and the tracking error is never larger than $\left|\delta_{\max }\right|=0.49^{\circ}$. Furthermore, the performances measures $J_{e}=0.001$ and $J_{m}=0.2120$ were obtained.

The CSF needs a relatively large number of delays, given the fact that the non-minimum phase zeros are located right on the stability border. Therefore, the pole radius was adjusted and set to $R=0.9999$, i.e. the CSF is supposed to just stabilize the inverse filter. With this new $R$, the CSF algorithm only needs $s=3$ additional advances. This siginficantly improves the tracking performance of the CSF: the precison tracking bandwidth is extended to $\omega_{b}=14.2610 \frac{\mathrm{rad}}{\mathrm{s}}$, while the other performance measures shrank to $J_{e}=2.9685 \times 10^{-5}$ and $J_{m}=2.7230 \times 10^{-4}$, respectively. The tracking error is now upper bounded by $\left|\delta_{\max }\right|=0.04^{\circ}$.

For this system, stabilization of the inverse filter with the help of feedback via a $d l q r$ design proved to be quite successful (see Section 4.1). As weighting matrices, $\mathbf{Q}=10^{-10} \cdot \mathbf{I}_{n_{c}}$ and $\mathbf{S}=100 \cdot \mathbf{I}_{p}$ were chosen. Since $r=2$ delays are still 
needed to invert the system, the cascade of CSF and feedback tracking system is an approximate delay of $r=2$ samples. With the $d l q r$ design, perfect tracking should be achieved over the whole considered frequency grid, $\omega_{b} \geq 500 \frac{\mathrm{rad}}{\mathrm{s}}$. The performance measures are much smaller as well, they become $J_{e}=1.4294 \times 10^{-7}$ and $J_{m}=2.2870 \times 10^{-5}$.

Clearly, this result is due to the ideal circumstances of the simulation. Therefore, an observer was added to the system and uncertainties were simulated. A $d l q r$ design on the system $\left(\boldsymbol{\Phi}^{\mathrm{T}}, \mathbf{C}^{\mathrm{T}}\right)$ was used to calculate the desired observer pole locations, with $\mathbf{Q}=10^{3} \cdot \mathbf{I}_{n}$ and $\mathbf{S}=\mathbf{I}_{p}$ as weighting matrices. Then, the algorithm from [27] was applied to obtain the observer gain matrix $\mathbf{L}$ with optimized stability robustness. The stability robustness bounds reduced to $\delta_{1}=0.3981$ and $\delta_{2}=0.5011$ in comparison to the non observer-based tracking system. As for the uncertainty, the inverse filter was based on the nominal plant model, but for the simulation, every entry of the plant's state-transition matrix was changed by $0.5 \%$, i.e. $\widetilde{\boldsymbol{\Phi}}=(1+\Delta) \boldsymbol{\Phi}$ and $\Delta=0.005$.

As to be expected, adding an observer and uncertainties aggravated the tracking performance. The best result was obtained with a frequency optimization approach, where $s=7$ additional delays were added before the design of the feedback gain matrix $\mathbf{F}$, i.e. the tracking system is an approximate delay of $d=9$ samples. The cost function (4.50) was used for the off-diagonal elements and (4.56) as penalty function for the stability constraint, with $\alpha=10$ and $\beta=10^{4}$. The precision tracking bandwidth dropped down to $\omega_{b}=0.0439 \frac{\mathrm{rad}}{\mathrm{s}}$, the performance measures became $J_{e}=1.5939$ and $J_{m}=3.8149$. Even though the precision tracking bandwidth reduced significantly, a sufficient tracking performance may still have been achieved for the desired reference trajectory. The tracking errors at the end of the rising and falling edges are way larger than before, $\left|\delta_{\max }\right| \approx 3.5^{\circ}$, and 


\begin{tabular}{c|cccccc} 
& CSF & CSF - dlqr & CSF - uncert. & IMP & IMP - dlqr & IMP - uncert. \\
\hline$\omega_{b}$ in $\frac{\mathrm{rad}}{\mathrm{s}}$ & 3.2099 & $\geq 500$ & 0.0439 & 0.8475 & $\geq 500$ & 0.0461 \\
$J_{e}$ & 0.001 & $1.4294 \times 10^{-7}$ & 1.5939 & 0.0446 & $3.9575 \times 10^{-21}$ & 1.6945 \\
$J_{m}$ & 0.212 & $2.2870 \times 10^{-5}$ & 3.8149 & 0.2820 & $9.3296 \times 10^{-21}$ & 3.9381
\end{tabular}

Table 5.6: Multilink Flexible Manipulator tracking results

it has to be evaluated on the physical system if that error is acceptable or not.

\section{Inverse Modified Plant}

The spectral radius of the modified plant is $\rho\left(\mathbf{\Phi}_{c}\right)=0.9888$, the corresponding pole radius is $R=0.9944$, and the IMP needs $s=14$ additional advances. The result achieved with this pole radius is slightly worse than the initial CSF result. The precision tracking bandwidth is $\omega_{b}=0.8475 \frac{\mathrm{rad}}{\mathrm{s}}$, while $J_{e}=0.0446$ and $J_{m}=$ 0.2820 holds.

Again, changing the pole radius to $R=0.9999$ yields better results (only $s=1$ additional advance is required), but the best result is obtained with a feedback approach via a dlqr design. As in the CSF case, the entire considered frequency grid belongs to the precision tracking bandwidth $\left(\omega_{b} \geq 500 \frac{\mathrm{rad}}{\mathrm{s}}\right)$, and the performance measures almost completely disappear: $J_{e}=3.9575 \times 10^{-21}$ and $J_{m}=9.3296 \times 10^{-21}$. Then, the same changes were made to the simulation settings as in the previous section, i.e. an observer and plant uncertainties were added. In the IMP case, the best result is achieved when $s=3$ additional advances are added before the frequency optimization. The precison tracking bandwidth and the performance measures are comparable to the CSF case.

The results are summarized in Table 5.6.

\section{Comparison to Source}

In [43], a continuous-time feedforward filter is proposed, in a closed-loop inversion feedforward architecture (see Fig. 2.1). Similar to the feedback approach in Section 3.2.3, an additive term is added to the inverse control law to move the 
eigenvalues of the inverse filter into the stability region. Several filter parameters $\kappa$ were considered (see $(5.41))$. For high values of $\kappa($ e.g. $\kappa=0.7)$, the authors detected that the inverse filter could not improve the tracking in comparison to non-filter based tracking. For small values (e.g. $\kappa=0.2$ ), the output trajectories settled faster to the desired angles (first a step to $20^{\circ}$, and then return back to $0^{\circ}$ ) and are also closer to the desired trajectory. For these filter parameters, however, the tracking system produces overshoot. For $\kappa=0.2$, the overshoot is around $35 \%$ on the worst channel $\left(27^{\circ}\right.$ instead of the desired $\left.20^{\circ}\right)$, and when the manipulator is supposed to return to the initial $0^{\circ}$ angles, the worst output "shoots over" up to almost $-10^{\circ}$. In this regard, a clear enhancement can be seen in the simulation results obtained by the CSF and IMP, as no overshooting is noticeable when the nominal plant is used in the simulations. When uncertainties were added to the plant model, the absolute tracking error was still smaller than $10^{\circ}$. Furthermore, the control effort is comparable for $\kappa=0.2$ for the inverse filter proposed in [43] and the CSF and IMP filters discussed in the previous subsection. Clearly, it has to be mentioned that in [43], actual experimental results are considered while in this thesis, only simulations were carried out.

\subsection{Overhead Crane}

In [44], a linearized model for an overhead crane is presented. It consists of a cart and a load, which is connected to the cart via a rope. The model (linearized around the origin of the state-space) is given as the continuous-time state-space 


\begin{tabular}{lll} 
Parameter & Symbol & Value \\
\hline cart friction & $c_{1}$ & $85 \frac{\mathrm{Nm}}{\mathrm{s}}$ \\
load friction & $c_{2}$ & $2.6 \frac{\mathrm{kgm}^{2}}{\mathrm{~s}}$ \\
load mass & $m$ & $20 \mathrm{~kg}$ \\
cart mass & $M$ & $38 \mathrm{~kg}$ \\
nominal rope length & $l$ & $1.61 \mathrm{~m}$
\end{tabular}

Table 5.7: Overhead Crane parameters

system $(\mathbf{A}, \mathbf{B}, \mathbf{C})$

$$
\begin{aligned}
& \mathbf{A}=\left[\begin{array}{cccc}
0 & 1 & 0 & 0 \\
0 & \frac{-c_{1}}{M} & \frac{m g}{M} & \frac{-c_{2}}{M} \\
0 & 0 & 0 & 1 \\
0 & \frac{c_{1}}{M l} & \frac{-g(m+M)}{M l} & \frac{(M+m) c_{2}}{M m l^{2}}
\end{array}\right] \\
& \mathbf{B}=\left[\begin{array}{c}
0 \\
\frac{1}{M} \\
0 \\
-\frac{1}{M l}
\end{array}\right] \\
& \mathbf{C}=\left[\begin{array}{llll}
1 & 0 & l & 0
\end{array}\right]
\end{aligned}
$$

where the input $u$ is the force applied to the cart and the output $y$ is the horizontal position of the suspended load. The parameters of the system are given in Table 5.7 , see [44] for more details.

The poles of the feedback tracking system were based on the continuous-time poles of the plant, which are located at

$$
\begin{aligned}
s_{1,2} & =-0.2650 \pm j 2.8792 \\
s_{3} & =-1.6303 \\
s_{4} & =0
\end{aligned}
$$

The design model (3.8) has an extra pole in 0, resulting from the integral additional dynamics.

For the design of the feedback tracking system, a settling time of $T_{S}=0.5 \mathrm{~s}$ is desired, which leads to $T=5 \mathrm{~ms}$ as the choice for the sampling time. Taking the guidelines in [27] into account, damping is added to the complex pole pair $s_{1,2}$, and the third order Bessel poles (see Table 5.1) were used for the remaining three 
poles. After the poles were mapped to discrete-time and the feedback gain $\mathbf{K}_{d}$ was calculated with place, the resulting (digital) feedback tracking system has

$$
\begin{aligned}
\lambda_{1,2} & =0.9604 \pm j 0.03641 \\
\lambda_{3,4} & =0.9548 \pm j 0.0137 \\
\lambda_{5} & =0.9511
\end{aligned}
$$

as eigenvalues and

$$
\begin{aligned}
& \eta_{1}=2.4452 \\
& \eta_{2}=-2.9112 \\
& \eta_{3}=-0.2202
\end{aligned}
$$

as zeros. Hence, the (digital) feedback tracking system has two non-minimum phase zeros, $\eta_{1}$ and $\eta_{2}$. The stability robustness bounds are $\delta_{1}=0.6404$ and $\delta_{2}=0.9006$.

The reference input should cause the center of gravity of the suspended crane load to move by $300 \mathrm{~mm}$, in a time interval $\tau=2 \mathrm{~s}$. In [44], a $\tau$-parameterized transition polynomial is proposed as the desired output function. The normalized reference input is given as

$$
\bar{r}(t ; \tau)= \begin{cases}0 & \text { if } t<0 \\ \frac{(2 n+1) !}{n ! \tau^{2 n+1}} \sum_{k=0}^{n} \frac{(-1)^{n-k} \tau^{k} t^{2 n-k+1}}{k !(n-k) !(2 n-k+1)} & \text { if } 0 \leq t \leq \tau \\ 1 & \text { if } t>\tau\end{cases}
$$

and it allows "an arbitrarily smooth transition between 0 and 1", and it can be shown that $\bar{r}(t ; \tau) \in \mathcal{C}^{(n)}$ [44]. In this thesis, $n$ was set to $n=2$, the resulting trajectory is shown in Fig. 5.19.

\section{Command Shaping Filter}

The feedback tracking system can be inverted exactly with a delay of $r=2$ samples. The pole radius for the inversion algorithm is set to $R=0.9806$, for the 


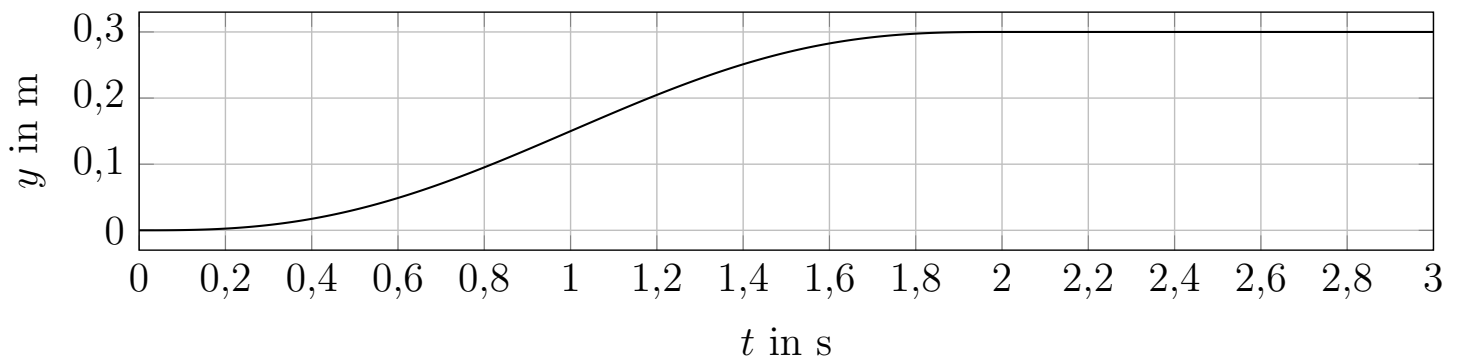

Figure 5.19: Overhead crane reference trajectory

spectral radius is $\rho\left(\mathbf{\Phi}_{c}\right)=0.9611$ in this case. The CSF algorithm needs $s=5$ additional advances to terminate; that is the filter-based tracking system is an approximate delay of $d=7$ samples. The resulting precision tracking bandwidth is $\omega_{b}=62.0089 \frac{\mathrm{rad}}{\mathrm{s}}, 99.27 \%$ of the reference input's energy is contained up to this frequency. The (absolute) tracking error has a peak of $\left|\delta_{\max }\right|=4.8 \times 10^{-6} \mathrm{~m}$ at the beginning of the reference input at $k T=0.025 \mathrm{~s}$. After this initial peak, the error stabilizes itself at around zero (it ranges in between $|\delta|=1 \times 10^{-9} \mathrm{~m}$ and $|\delta|=1 \times 10^{-12} \mathrm{~m}$ ) during the rising edge of the reference input. At the end of the rising edge at $k T \approx 2 \mathrm{~s}$, the tracking error has another (little) peak with $|\delta|=7.7 \times 10^{-8} \mathrm{~m}$. The resulting performance measures are $J_{e}=4.4344 \times 10^{-10}$ and $J_{m}=2.5234 \times 10^{-8}$.

The best optimization result is attained when (4.56) is used as penalty function for the stability constraint and $s=s_{\max }=5$ additional advances are considered, with a weight of $\beta=10^{4}$ and $\omega_{N}=5.7309 \frac{\mathrm{rad}}{\mathrm{s}}$. This approach, however, does not deliver significantly better results.

\section{Inverse Modified Plant}

The modified plant has a spectral radius of $\rho\left(\boldsymbol{\Phi}_{c}\right)=0.9717$, resulting in $R=0.9859$ as the pole radius. The modified plant can be exactly inverted with $r=1$ delay, and the IMP algorithm needs $s=4$ additional advances to keep the 


\begin{tabular}{c|cccc} 
& CSF & CSF - freq. opt. & IMP & IMP - freq. opt. \\
\hline$\omega_{b}$ in $\frac{\mathrm{rad}}{\mathrm{s}}$ & 62.0089 & 62.1108 & 57.7365 & 57.7365 \\
$J_{e}$ & $4.4344 \times 10^{-11}$ & $4.4342 \times 10^{-11}$ & $2.8911 \times 10^{-11}$ & $2.8911 \times 10^{-11}$ \\
$J_{m}$ & $2.5234 \times 10^{-8}$ & $2.5276 \times 10^{-8}$ & $1.6488 \times 10^{-9}$ & $1.6488 \times 10^{-9}$
\end{tabular}

Table 5.8: Overhead Crane tracking results

pole radius. Thus, the tracking system becomes an approximate delay of $d=5$ samples, while $\omega_{b}=57.7365 \frac{\mathrm{rad}}{\mathrm{s}}$ holds for the actual precision tracking bandwidth, which is somewhat worse than the CSF result, but this bandwidth still contains the same energy as the CSF bandwidth.

Just as the CSF result, the IMP tracking error has a peak at the beginning of the reference trajectory, with $\left|\delta_{\max }\right|=1.2 \times 10^{-6} \mathrm{~m}$, and it has another (smaller) peak at the end of the reference with $|\delta|=1.2 \times 10^{-7} \mathrm{~m}$. The performance measures become $J_{e}=2.8911 \times 10^{-11}$ and $J_{m}=1.6488 \times 10^{-9}$.

Again, the best optimization result can be observed if the maximum number of additional advances is considered, $s=s_{\max }=4$, but no noticeable improvement is obtained.

Finally, all the tracking results are shown in Table 5.8.

\section{Comparison to Source}

In [44], a dynamic inversion technique is presented by the authors to design suitable position (and velocity) set-point feedforward signals. In their approach, they apply a continuous-time input-output inversion and are able to get rid of the postaction (postaction means that the invertng signal reaches its steady-state after the transition time), which usually occurs as a problem in motion control inversion problems and interferes with the practicability of these approaches [44].

Besides the desired output trajectory, [44] specifies limitations for the control input $u$. The cart is actuated with a brushless servomotor through a pulley and toothed belt system, and the relationship between the motor torque $T$ and the 
force applied to the cart $u$ (the input signal of the plant model) is given by

$$
u=\eta r i T
$$

where $i$ is the reduction ratio of an epicycloidal speed reducer, $\eta$ its mechanical efficiency and $r$ the pulley radius [44]. The maximum continuous motor torque is $T_{\max }=3 \mathrm{Nm}$, and the peak is at $T_{\text {peak }}=6 \mathrm{Nm}$. It is noted, however, that there has to be a typing error in (5.47) (for example because the units are not consistent, $u$ is in Newtons, $r$ in meters and $T$ in Newton-meters, so $[r T]=\mathrm{Nm}^{2}$ holds for the unit of the product of torque and radius). It is assumed that at least the reciprocal of $r$ has to be used in (5.47) (the units are consistent then), but it is not known whether the reciprocals of $\eta$ and $i$ have to be taken as well. Anyway, the motor torque following the CSF and IMP design is larger than $T_{\text {peak }}$, but it cannot be evaluated if this would be the case for an experiment on the real physical system as well, since it is unknown how (5.47) has to be modified to adequately describe the system's dynamics.

A similar behaviour as in the CSF and IMP cases for the tracking error can be seen in the results presented in [44] for the position control, i.e. it has a relatively sharp peak at the beginning of the reference trajectory and converges towards zero in the course of the tracking process. Its maximum value is at around $\left|\delta_{\max }\right|=$ $0.8 \mathrm{~mm}$, which is worse than the CSF results of $\left|\delta_{\max }\right|=4.8 \mu \mathrm{m}$. Naturally, the tracking results are hard to compare since they were obtained from an experimental setup in [44], while simulations were used in this thesis.

\subsection{Two Discs}

In [15], a flexible structure consisting of two discs which are connected by a thin freely rotating shaft is considered for tracking. The input is the voltage $U(t)$ applied to a DC motor, while the output is the angular rotation (in degrees) of the second disc, $\theta_{2}$ (the disc further away from the motor). The plant is given as the 
continuous-time state-space model:

$$
\begin{aligned}
& \mathbf{A}=\left[\begin{array}{cccc}
0 & 1 & 0 & 0 \\
-3.656 & -0.436 & 3.573 & -0.091 \\
0 & 0 & 0 & 1 \\
3.245 & -0.126 & -3.259 & -0.076
\end{array}\right] \\
& \mathbf{B}=\left[\begin{array}{c}
0 \\
21.9027 \\
0 \\
3.588
\end{array}\right] \\
& \mathbf{C}=\left[\begin{array}{llll}
0 & 0 & 1 & 0
\end{array}\right] .
\end{aligned}
$$

The plant has the eigenvalues

$$
\begin{aligned}
& s_{1,2}=-0.077 \pm j 2.6131 \\
& s_{3,4}=-0.1783 \pm j 0.1229
\end{aligned}
$$

and a complex non-minimum phase zero pair at $z=0.1666 \pm j 4.8412$. The design model (3.8) has an extra pole in 0 , due to the integral additional dynamics. In this thesis, the continuous-time plant is discretized with a sampling time $T=0.3 \mathrm{~s}$. This values was chosen because a settling time of $T_{S}=30 \mathrm{~s}$ is desired for the feedback tracking system (Fig. 3.1). The feedback tracking system was designed as follows (according to the guidelines in [27]): the second eigenvalue pair $s_{3,4}$ is kept, and the remaining poles in the continuous-time domain are chosen as the third order Bessel poles (see Table 5.1). After these pole locations were mapped into the discrete-time domain using the $\mathrm{ZOH}$ pole-mapping formula, and place was used to calculate the feedback gain $\mathbf{K}_{d}$, the (digital) eigenvalues of the feedback tracking system are

$$
\begin{aligned}
& \lambda_{1,2}=0.6713 \pm j 0.6693 \quad \lambda_{5}=0.9511 \\
& \lambda_{3,4}=0.9604 \pm j 0.0364
\end{aligned}
$$

and

$$
\begin{aligned}
\eta_{1,2} & =0.1144 \pm j 1.0503 \\
\eta_{3} & =-0.8969
\end{aligned}
$$




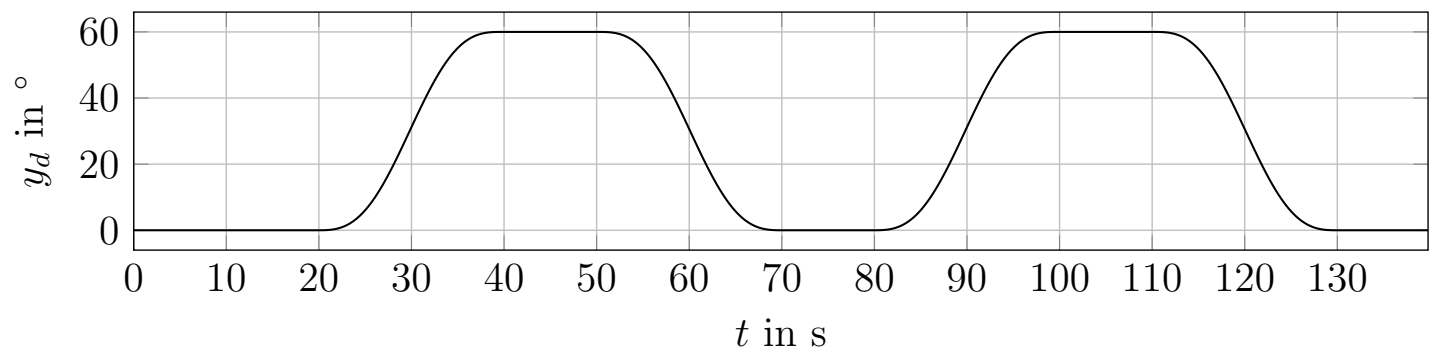

Figure 5.20: Two discs reference trajectory

holds for the zeros. The stability robustness bounds $\delta_{1}=1$ and $\delta_{2}=0.9251$ are reached. It is the task of the inversion algorithm to stabilize the non-minimum phase zero $\eta_{1,2}$. It was not possible to exactly replicate the reference trajectory that was used in the source, since in [15], it was generated on-line. The trajectory used in this thesis can be seen in Fig. 5.20.

\section{Command Shaping Filter}

The feedback tracking system can be exactly inverted with a delay of $r=2$ samples. The pole radius for the filter design was chosen to be $R=0.9806$, as $\rho\left(\boldsymbol{\Phi}_{c}\right)=0.9611$ holds. To attain this pole radius, $s=3$ additional advances are required, making the cascade of CSF and feedback tracking system an approximate delay of $d=5$ samples.

The CSF reaches a precision tracking bandwidth of $\omega_{b}=0.6842 \frac{\mathrm{rad}}{\mathrm{s}}$. After simulations have been carried out, it can be concluded that this bandwidth is sufficient: the tracking error is never higher than $\left|\delta_{\max }\right|=0.01^{\circ}$ for the desired reference. The performance measures are $J_{e}=1.0266 \times 10^{-5}$ and $J_{m}=2.0780 \times$ $10^{-6}$.

The optimization approach to design a stabilizing feedback matrix $\mathbf{F}$ can be used to enhance the tracking performance. When an unconstrained optimization with (4.56) as penalty function $\left(\beta=10^{4}\right)$ and $\omega_{N}=0.143 \frac{\mathrm{rad}}{\mathrm{s}}$ is used, where $\mathbf{F}_{0}$ is calculated with a dlqr design $\left(\mathbf{Q}=10^{-10} \cdot \mathbf{I}_{n_{c}}, \mathbf{S}=100 \cdot \mathbf{I}_{p}\right)$, the precision 
tracking bandwidth becomes $\omega_{b}=1.5239 \frac{\mathrm{rad}}{\mathrm{s}}$ which more than doubles the previous bandwidth. The maximum tracking error shrinks down to $\left|\delta_{\max }\right|=0.002^{\circ}$, and the performance measures become $J_{e}=1.5010 \times 10^{-6}$ and $J_{m}=1.2895 \times 10^{-7}$.

\section{Inverse Modified Plant}

The spectral radius of the modifed plant is $\rho\left(\boldsymbol{\Phi}_{c}\right)=0.9480$, correspondingly the pole radius becomes $R=0.9740$. While the modified plant can be inverted exactly with a delay of $r=1$ samples, it takes the algorithm $s=2$ more advances to move the filter eigenvalues inside the desired pole radius, which is why the filter-based tracking system is an approximate delay of $d=3$ samples.

The achieved precision tracking bandwidth is the same as for the initial CSF design, $\omega_{b}=0.6842 \frac{\mathrm{rad}}{\mathrm{s}}$, though the other performances measures differ from the CSF result. The maximum tracking error is slightly worse $\left(\left|\delta_{\max }\right|=0.016^{\circ}\right)$, but since the maximum value of the reference input is $r_{\max }=60^{\circ}$, this deviation is negligible. The performance measures $J_{e}$ and $J_{m}$ stay roughly the same.

As in the CSF case, slightly better results can be obtained if the frequency optimization approach is used to design a feedback matrix $\mathbf{F}$ to stabilize this filter. For the IMP, however, it proved to be the best choice to add $s=1$ additional delay and then design the feedback $\mathbf{F}$, i.e. the hybrid approach between adding delays and optimizing $\mathbf{F}$ is used (Section 3.2.3). The initial value is the same as in the CSF case (but naturally, the considered system is different). The most significant refinement in comparison to the standard IMP design is the precision tracking bandwidth, it could be expanded to $\omega_{b}=1.0943 \frac{\mathrm{rad}}{\mathrm{s}}$ (this is faintly worse than the CSF optimization result though).

The results are summarized in Table 5.9. 


\begin{tabular}{c|cccc} 
& CSF & CSF - freq. opt. & IMP & IMP - freq. opt. \\
\hline$\omega_{b}$ in $\frac{\mathrm{rad}}{\mathrm{s}}$ & 0.6842 & 1.5239 & 0.6842 & 1.0943 \\
$J_{e}$ & $1.0266 \times 10^{-5}$ & $1.5010 \times 10^{-6}$ & $8.3159 \times 10^{-5}$ & $8.3649 \times 10^{-6}$ \\
$J_{m}$ & $2.0780 \times 10^{-5}$ & $1.2895 \times 10^{-7}$ & $7.2629 \times 10^{-6}$ & $7.5322 \times 10^{-7}$
\end{tabular}

Table 5.9: Two Discs tracking results

\section{Comparison to the Source}

As mentioned earlier, a real comparison is difficult since the reference trajectory was created on-line in [15], so it is not exactly the same as the one shown in Fig. 5.20. Further, the results presented in [15] are experimental results, and not simulations.

In [15], the internal (or zero) dynamics are decoupled into a stable and unstable part, and bounded solutions are found for both parts . For the unstable dynamics, however, an infinite preview time is required (i.e. the desired output must be completely specified, which the author does not want to assume). Therefore, the solution for the unstable dynamics are approximated using a finite preview time $T_{p}$ (details can also be found in Section 2). Two experiments were carried out and presented, the first one uses a preview time of $T_{p}=20 \mathrm{~s}$, the second one uses $T_{p}=50 \mathrm{~s}$.

The tracking performance for the first preview time is rather bad, as large tracking errors can be observed during the rising edges of the reference trajectory. For the first rising edge, the output first goes down to $\theta_{2} \approx-20^{\circ}$, before it approaches the reference again. This behaviour vanishes for $T_{p}=50 \mathrm{~s}$, but still small tracking erros are present, this time during the falling edges (albeit they are neglectably small in comparison to the other preview time). Even though the preview time in [15] and the advances needed by the inversion algorithm in this thesis are hardly comparable, it can be noted that both the best CSF and MP results only need $d=2$ delays (i.e. a "preview" of $T_{p}=0.6 \mathrm{~s}$ ). 


\section{CHAPTER 6}

\section{Conclusion}

Methods to approximately invert linear non-minimum phase MIMO systems were presented. The success of the inversion techniques was confirmed with simulation results.

Two different tracking architectures were considered. The first one is the command shaping filter (CSF), shown in Fig. 3.5, which inverts a feedback-based tracking system to extend the precision tracking bandwidth. The second one is the inverse modified plant (IMP, see Fig. 3.6), which inverts a modified plant that is part of a feedback tracking system.

First, an approach to add advances in order to design a stable inverse was presented. This makes the cascade of the inverse filter and the system to be inverted an approximate decoupled system of pure delays. The inverse filter design was extended to a feedback approach to stabilize the filter, which led in general to a fewer number of required delays. Several possibilities to calculate the corresponding feedback gain matrix were discussed, among which especially a frequency optimization approach excelled.

An advantage of the IMP is that the additional dynamics of the feedback tracking system can be based on the reference trajectory's dynamics, which showed to be an improvement for some systems. However, this could also be interpreted as a disadvantage, since the CSF always got its best tracking results with "standard" integrator additional dynamics, thus the CSF performance apparently depends less on the chosen reference input and the architecture does not have to be changed if a different signal is desired to be tracked. It can be noted, though, that the IMP tends to achieve the larger precision tracking bandwidth. The CSF, however, has 
some practical advantages, e.g. the modified plant (which is inverted by the IMP algorithm) is not guaranteed to be stable.

An advantage of the "standard" way to compute the CSF and IMP (i.e. adding advances until the filter is stabilized) is a very easy implementation, and the derivation of this filter is based on a reasonable approximation. A disadvantage may be that the designer has a rather limited influence on the resulting tracking performance, as the only parameter he can change is the pole radius $R$. The design of the feedback tracking system has an influence on the tracking performance as well, but it is not clear how changes in $\left(\boldsymbol{\Phi}_{a}, \boldsymbol{\Gamma}_{a}\right)$ or $\mathbf{K}_{d}$ will translate to the tracking performance. On the other hand, if the frequency optimization approach is used, the designer has some more influence on the inverse filter, e.g. through the choice of the considered frequency grid, which allows him to a certain degree to affect the precision tracking bandwidth. Moreover, it is possible to test several values for the optimization parameters and different formulations for the objective function, as presented in Section 4.3. Clearly, the design of the inverse filter with this approach becomes an iterative task, which is more complicated than the standard design.

As a conclusion, it can be stated that the choice of the tracking architecture and the filter design technique depends on the tracking problem and the system under consideration; in the ideal case both architectures are tested with different design techniques in simulations and the best performance is selected.

\subsection{Areas of Future Work}

A problem that has to be further examined is the proof that the eigenvalues of the inverse filter (3.21c) contain the zeros of the discrete-time plant (3.2). In Section 3.2.1 it was assumed that (3.23) holds for the transmission zeros of a system, but as mentioned in Section 3.2.1, other definitions exist in the literature. It has to be evaluated when these two different definitions coincide, since it is 
thought that they do for the class of systems that were considered during this thesis. The results from Section 3.2.1 were confirmed by Matlab computations and simulations, i.e. the inverse filter (3.21c) always contained the zeros of the plant (3.2). Furthermore, an argument has to be given that the invariant zeros of system (3.63) include the invariant zeros of the plant (3.2) if the order of the additional dynamics is not equal to the number of plant outputs.

The optimization approach presented in Section 4.3 proved to be pretty successful in designing an inverse filter. In the current implementation, however, it does have some flaws. It takes a very long time until the optimization terminates, especially for high order systems like the Bell 205 Helicopter or the Multilink Flexible Manipulator. For that reason, the gradient of the objective function could be calculated and implemented in Matlab to speed up the optimization.

Further, the inversion methods may be tested on more example systems and experiments should be carried out to confirm the achieved success in the simulations. 


\section{LIST OF REFERENCES}

[1] J. T. Wen and B. Potsaid, "An experimental study of a high performance motion control system," in Proceedings of the 2004 American Control Conference, vol. 6, 2004, pp. 5158-5163.

[2] K. Åström, P. Hagander, and J. Sternby, "Zeros of sampled systems," Automatica, vol. 20, no. 1, pp. 31 - 38, 1984. [Online]. Available: http://www.sciencedirect.com/science/article/pii/0005109884900621

[3] Ying Wu and Qingze Zou, "Robust-inversion-based 2DOF-control design for output tracking: Piezoelectric actuator example," in 2007 46th IEEE Conference on Decision and Control, 2007, pp. 2451-2457.

[4] L. Y. Pao, J. A. Butterworth, and D. Y. Abramovitch, "Combined Feedforward/Feedback Control of Atomic Force Microscopes," in 2007 American Control Conference, 2007, pp. 3509-3515.

[5] J. A. Butterworth, L. Y. Pao, and D. Y. Abramovitch, "The effect of nonminimum-phase zero locations on the performance of feedforward modelinverse control techniques in discrete-time systems," in 2008 American Control Conference, 2008, pp. 2696-2702.

[6] Qingze Zou and S. Devasia, "Preview-based optimal inversion for output tracking: application to scanning tunneling microscopy," IEEE Transactions on Control Systems Technology, vol. 12, no. 3, pp. 375-386, 2004.

[7] S. S. Aphale, S. Devasia, and S. O. R. Moheimani, "Achieving high-bandwidth nanopositioning in presence of plant uncertainties," in 2008 IEEE/ASME International Conference on Advanced Intelligent Mechatronics, 2008, pp. 943948.

[8] K. Leang, Q. Zou, and S. Devasia, "Feedforward control of piezoactuators in atomic force microscope systems," IEEE Control Systems, vol. 29, pp. 70-82, 2009.

[9] M. W. Oppenheimer and D. B. Doman, "Control of an unstable, nonminimum phase hypersonic vehicle model," in 2006 IEEE Aerospace Conference, 2006, pp. 7 pp.-.

[10] D. Y. Abramovitch, S. B. Andersson, L. Y. Pao, and G. Schitter, "A Tutorial on the Mechanisms, Dynamics, and Control of Atomic Force Microscopes," in 2007 American Control Conference, 2007, pp. 3488-3502. 
[11] L. Dai, X. Li, Y. Zhu, and M. Zhang, "Quantitative Analysis on Tracking Error Under Different Control Architectures and Feedforward Methods," in 2019 American Control Conference (ACC), 2019, pp. 5680-5686.

[12] B. P. Rigney, L. Y. Pao, and D. A. Lawrence, "Nonminimum Phase Dynamic Inversion for Settle Time Applications," IEEE Transactions on Control Systems Technology, vol. 17, no. 5, pp. 989-1005, 2009.

[13] J. A. Butterworth, L. Y. Pao, and D. Y. Abramovitch, "The effect of nonminimum-phase zero locations on the performance of feedforward modelinverse control techniques in discrete-time systems," in 2008 American Control Conference, 2008, pp. 2696-2702.

[14] M. Tomizuka, "Zero Phase Error Tracking Algorithm for Digital Control," Journal of Dynamic Systems, Measurement, and Control, vol. 109, no. 1, pp. 65-68, 03 1987. [Online]. Available: https://doi.org/10.1115/1.3143822

[15] Q. Zou and S. Devasia, "Preview-based stable-inversion for output tracking," in Proceedings of the 1999 American Control Conference (Cat. No. 99CH36251), vol. 5, 1999, pp. 3544-3548 vol.5.

[16] G. Marro, D. Prattichizzo, and E. Zattoni, "Convolution profiles for right inversion of multivariable non-minimum phase discrete-time systems," Automatica, vol. 38, pp. 1695-1703, 102002.

[17] T. Shiraishi and H. Fujimoto, "Trajectory tracking control method based on zero-phase minimum-phase factorization for nonminimum-phase continuoustime system," in 2012 12th IEEE International Workshop on Advanced Motion Control (AMC), 2012, pp. 1-6.

[18] L. Jetto, V. Orsini, and R. Romagnoli, "Optimal transient performance under output set-point reset," International Journal of Robust and Nonlinear Control, vol. 26, no. 13, pp. 2788-2806, 2016. [Online]. Available: https://onlinelibrary.wiley.com/doi/abs/10.1002/rnc.3475

[19] S. Devasia, "Output Tracking with Nonhyperbolic and Near Nonhyperbolic Internal Dynamics: Helicopter Hover Control," Journal of Guidance, Control, and Dynamics, vol. 20, no. 3, pp. 573-580, 1997. [Online]. Available: https://doi.org/10.2514/2.4079

[20] M. M. Michałek, "Simple causal fixed-structure feedforward control law for general continuous-time LTI SISO systems," in 2014 European Control Conference (ECC), 2014, pp. 61-66.

[21] T.-C. Tsao and M. Tomizuka, "Adaptive Zero Phase Error Tracking Algorithm for Digital Control," Journal of Dynamic Systems, Measurement, and Control, vol. 109, no. 4, pp. 349-354, 12 1987. [Online]. Available: https://doi.org/10.1115/1.3143866 
[22] H. Fujimoto, Y. Hori, and A. Kawamura, "Perfect tracking control based on multirate feedforward control with generalized sampling periods," IEEE Transactions on Industrial Electronics, vol. 48, no. 3, pp. 636-644, 2001.

[23] B. Potsaid and J. T. Wen, "High performance motion tracking control," in Proceedings of the 2004 IEEE International Conference on Control Applications, 2004., vol. 1, 2004, pp. 718-723 Vol.1.

[24] R. J. Vaccaro, Y. Boudria, M. Jouaneh, and A. S. Zaki, "Precision Tracking for an H-Frame XY Positioning System," 2015, unpublished.

[25] R. J. Vaccaro, Y. Boudria, and M. Jouaneh, "Inversion of MIMO Control Systems for Precision Tracking," 2014, unpublished.

[26] R. J. Vaccaro, Digital Control: A State-Space Approach. New York, NY: McGraw-Hill, 1995.

[27] R. J. Vaccaro, "An optimization approach to the pole-placement design of robust linear multivariable control systems," in 2014 American Control Conference, 2014, pp. 4298-4305.

[28] R. J. Vaccaro, "Stability Robustness," 2018, unpublished.

[29] A. Emami-Naeini and P. V. Dooren], "Computation of zeros of linear multivariable systems," Automatica, vol. 18, no. 4, pp. 415 - 430, 1982. [Online]. Available: http://www.sciencedirect.com/science/article/pii/ 000510988290070X

[30] J. Lunze, Regelungstechnik 2, 7th ed. Springer, 2013.

[31] G. Roppenecker and H.-P. Preuss, "Übersichtsaufsatz / Survey Paper: Nullstellen und Pole linearer Mehrgrößensysteme Teil 1: Grundlagen und Definitionen / Zeros and poles of linear multivariable systems Part 1: Basic concepts and definitions," at - Automatisierungstechnik, vol. 30, no. 1-12, pp. 219 - 225, 1982. [Online]. Available: https: //www.degruyter.com/view/journals/auto/30/1-12/article-p219.xml

[32] A. MacFarlane and N. Karcanias, "Poles and zeros of linear multivariable systems: A survey of the algebraic, geometric and complex-variable theory," International Journal of Control - INT J CONTR, vol. 24, pp. 33-74, 071976.

[33] K. M. Moudgalya, Digital control. Chichester, England: John Wiley \& Sons, Ltd, 2007.

[34] G. H. Golub and C. F. Van Loan, Matrix Computations, 4th ed. The Johns Hopkins University Press, 2013. 
[35] C.-T. Chen, Linear System Theory and Design, 3rd ed. USA: Oxford University Press, Inc., 1998.

[36] K. Zhou and J. C. Doyle, Essentials of Robust Control. Prentice-Hall, 1998.

[37] J. Lunze, Regelungstechnik 1, 11st ed. Springer, 2016.

[38] S. Boyd and L. Vandenberghe, Convex Optimization. USA: Cambridge University Press, 2004.

[39] U. Konigorski, "Entwurf robuster strukturbeschränkter Zustandsregelungen durch Polgebietsvorgabe mittels Straffunktionen / Design of robust structurally-constrained state feedback controllers by pole region assignment using penalty functions," at - Automatisierungstechnik, vol. 35, no. 1-12, pp. 254 - 258, 1987. [Online]. Available: https://www.degruyter.com/view/ journals/auto/35/1-12/article-p254.xml

[40] J. O. Smith, Introduction to Digital Filters with Audio Applications. http://ccrma.stanford.edu/ jos/filters/, accessed 07/03/2020, online book.

[41] A. V. Oppenheim, R. W. Schafer, and J. R. Buck, Discrete-Time Signal Processing, 2nd ed. USA: Prentice-Hall, Inc., 1999.

[42] K. S. Sollmann, M. K. Jouaneh, and D. Lavender, "Dynamic Modeling of a Two-Axis, Parallel, H-Frame-Type XY Positioning System," IEEE/ASME Transactions on Mechatronics, vol. 15, no. 2, pp. 280-290, 2010.

[43] W. Njeri, M. Sasaki, and K. Matsushita, "Enhanced vibration control of a multilink flexible manipulator using filtered inverse controller," ROBOMECH Journal, vol. 5, no. 28, 2018.

[44] F. Padula, A. Visioli, D. Facchinetti, and A. Saleri, "A dynamic inversion approach for oscillation-free control of overhead cranes," in 2015 IEEE 20th Conference on Emerging Technologies Factory Automation (ETFA), 2015, pp. $1-6$.

[45] S. Ross, A First Course in Probability, 8th ed. Pearson Prentice Hall, 2010. 


\section{APPENDIX}

\section{Derivation of the Dual Problem}

In this appendix, detailed calculations for the derivation of the Lagrange dual function are presented (see Section 4.2.2).

First, the inequality (4.21), (4.20) and equality (4.14), (4.15), (4.18) constraints that were included in the optimization problem shall be expressed in terms of the decision variable $\boldsymbol{x}$. As a reminder, $\boldsymbol{x}$ is defined as

$$
\boldsymbol{x}=\left[\begin{array}{c}
\hat{\boldsymbol{f}}_{1} \\
\vdots \\
\hat{\boldsymbol{f}}_{n_{z}}
\end{array}\right]
$$

i.e. the first $n_{z}$ columns of $\hat{\mathbf{F}}$. For better readability, the bigger part of these calculations is omitted (because they just involve a lot of indexing) and only the results are presented. Note that $\boldsymbol{\gamma}_{\psi, j}^{\mathrm{T}}$ denotes the $j$ th row of matrix $\boldsymbol{\Gamma}_{\psi}$ defined in (4.8c), $n_{i}$ the number of complex non-minimum-phase zeros and $n_{z}$ the number of all non-minimum phase zeros (see Section 4.2.1).

First, the constraint (4.14) is considered. It can be expressed as

$$
\left[\begin{array}{c}
\boldsymbol{\Gamma}_{1} \mathbf{T}_{1} \\
\vdots \\
\boldsymbol{\Gamma}_{n_{i}} \mathbf{T}_{n_{i}}
\end{array}\right] \boldsymbol{x}=\mathbf{0}
$$

with (blkdiag refers to the Matlab function of the same name):

$$
\begin{aligned}
& \boldsymbol{\Gamma}_{i}=\left[\begin{array}{c}
\operatorname{blkdiag}(\overbrace{\boldsymbol{\gamma}_{\psi, 2 i-1}^{\mathrm{T}}, \ldots, \boldsymbol{\gamma}_{\psi, 2 i-1}^{\mathrm{T}}}^{n_{z}-2 \text { times }}) \\
\operatorname{blkdiag}(\underbrace{\gamma_{\psi, 2 i}^{\mathrm{T}}, \ldots, \boldsymbol{\gamma}_{\psi, 2 i}^{\mathrm{T}}}_{n_{z}-2 \text { times }})
\end{array}\right] \\
& \mathbf{T}_{i}=\left[\begin{array}{ll}
\mathbf{I}_{p(2 i-2)} & {\left[\begin{array}{ll}
\mathbf{0}_{p\left(n_{z}-2 i\right) \times 2 p} & \mathbf{I}_{p\left(n_{z}-2 i\right)}
\end{array}\right] .}
\end{array}\right.
\end{aligned}
$$


Next, we care about the constraint (4.15). We get

$$
\left[\begin{array}{c}
\widetilde{\boldsymbol{\Gamma}}_{1} \widetilde{\mathbf{T}}_{1} \\
\vdots \\
\widetilde{\boldsymbol{\Gamma}}_{n_{i}} \widetilde{\mathbf{T}}_{n_{i}}
\end{array}\right] \boldsymbol{x}=\mathbf{0}
$$

with

$$
\begin{aligned}
& \widetilde{\boldsymbol{\Gamma}}_{i}=\operatorname{blkdiag}(\overbrace{\gamma_{\psi, 2 n_{i}+i}^{\mathrm{T}}, \ldots, \boldsymbol{\gamma}_{\psi, 2 n_{i}+i}^{\mathrm{T}}}^{n_{z}-1 \text { times }})
\end{aligned}
$$

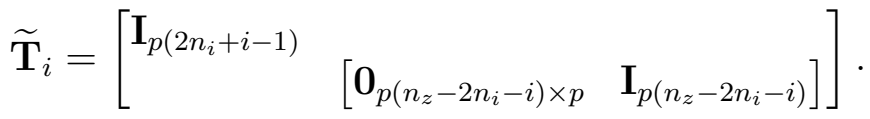

Finally, we write (4.18) as

$$
\left[\begin{array}{c}
\hat{\boldsymbol{\Gamma}}_{1} \hat{\mathbf{T}}_{1} \\
\vdots \\
\hat{\boldsymbol{\Gamma}}_{n_{i}} \hat{\mathbf{T}}_{n_{i}}
\end{array}\right] \boldsymbol{x}=\mathbf{0}
$$

with

$$
\begin{aligned}
& \hat{\boldsymbol{\Gamma}}_{i}=\left[\begin{array}{cc}
\boldsymbol{\gamma}_{\psi, 2 i-1}^{\mathrm{T}} & -\boldsymbol{\gamma}_{\psi, 2 i}^{\mathrm{T}} \\
\boldsymbol{\gamma}_{\psi, 2 i}^{\mathrm{T}} & \boldsymbol{\gamma}_{\psi, 2 i-1}^{\mathrm{T}}
\end{array}\right] \\
& \hat{\mathbf{T}}_{i}=\left[\begin{array}{ll}
{\left[\begin{array}{ll}
\mathbf{0}_{p \times p(2 i-2)} & \mathbf{I}_{p}
\end{array}\right]} & {\left[\begin{array}{ll}
\mathbf{I}_{p} & \mathbf{0}_{p \times p\left(n_{z}-2 i\right)}
\end{array}\right] .}
\end{array}\right.
\end{aligned}
$$

As a next step, we consider the inequality constraints. The calculations are presented in more detail here, since the absolute value is a nonlinear function and is therefore harder to find an expression in terms of $\boldsymbol{x}$. First, we take a look at (4.21). This is the constraint that the real-valued unstable eigenvalues have to be moved into the unit circle. Since (4.21) is the absolute value of a real number, we can rewrite it as

$$
-1<\lambda_{j}-\gamma_{\psi, j} \hat{\boldsymbol{f}}_{j}<1 \quad j=2 n_{i}+1,2 n_{i}+2, \ldots, n_{z}
$$

which gives us the two demands

$$
\begin{array}{r}
\lambda_{j}-\boldsymbol{\gamma}_{\psi, j}^{\mathrm{T}} \hat{\boldsymbol{f}}_{j}-1<0 \\
-\lambda_{j}+\boldsymbol{\gamma}_{\psi, j}^{\mathrm{T}} \hat{\boldsymbol{f}}_{j}-1<0
\end{array}
$$


for each $j$ which can be expressed as

$$
\mathrm{MN} \boldsymbol{x}+\boldsymbol{k}<\mathbf{0}
$$

where

$$
\begin{aligned}
\mathbf{M} & =\left[\begin{array}{ccc}
-\gamma_{\psi, 2 n_{i}+1}^{\mathrm{T}} & & \\
& \ddots & \\
& & -\boldsymbol{\gamma}_{\psi, n_{z}}^{\mathrm{T}} \\
\boldsymbol{\gamma}_{\psi, 2 n_{i}+1}^{\mathrm{T}} & & \\
& \ddots & \\
& & \boldsymbol{\gamma}_{\psi, n_{z}}^{\mathrm{T}}
\end{array}\right] \\
\mathbf{N} & =\left[\begin{array}{cc}
\mathbf{0}_{p\left(n_{z}-2 n_{i}\right) \times 2 p n_{i}} & \mathbf{I}_{p\left(n_{z}-2 n_{i}\right)}
\end{array}\right] \\
\boldsymbol{k}= & {\left[\begin{array}{c}
\lambda_{2 n_{i}+1}-1 \\
\vdots \\
\lambda_{n_{z}}-1 \\
-\left(\lambda_{2 n_{i}+1}+1\right) \\
\vdots \\
-\left(\lambda_{n_{z}}+1\right)
\end{array}\right] . }
\end{aligned}
$$

Finally, the constraints for the complex eigenvalues (4.20) have to be considered. We rewrite $(4.20)$ as

$$
\sqrt{\delta_{j}^{2}-2 \delta_{j} \boldsymbol{\gamma}_{\psi, j}^{\mathrm{T}} \hat{\boldsymbol{f}}_{j}+\left(\boldsymbol{\gamma}_{\psi, j}^{\mathrm{T}} \hat{\boldsymbol{f}}_{j}\right)^{2}+\omega_{j}^{2}-2 \omega_{j} \boldsymbol{\gamma}_{\psi, j}^{\mathrm{T}} \hat{\boldsymbol{f}}_{j+1}+\left(\boldsymbol{\gamma}_{\psi, j}^{\mathrm{T}} \hat{\boldsymbol{f}}_{j+1}\right)^{2}}<1
$$

which is fulfilled if

$$
\delta_{j}^{2}-2 \delta_{j} \boldsymbol{\gamma}_{\psi, j}^{\mathrm{T}} \hat{\boldsymbol{f}}_{j}+\left(\boldsymbol{\gamma}_{\psi, j}^{\mathrm{T}} \hat{\boldsymbol{f}}_{j}\right)^{2}+\omega_{j}^{2}-2 \omega_{j} \boldsymbol{\gamma}_{\psi, j}^{\mathrm{T}} \hat{\boldsymbol{f}}_{j+1}+\left(\boldsymbol{\gamma}_{\psi, j}^{\mathrm{T}} \hat{\boldsymbol{f}}_{j+1}\right)^{2}-1<0
$$

holds. Because of

$$
\begin{aligned}
\boldsymbol{\gamma}_{\psi, j}^{\mathrm{T}} & =\left[\begin{array}{ccc}
\gamma_{j 1} & \ldots & \gamma_{j p}
\end{array}\right] \\
\hat{\boldsymbol{f}}_{j} & =\left[\begin{array}{c}
\hat{f}_{1 j} \\
\vdots \\
\hat{f}_{p j}
\end{array}\right]
\end{aligned}
$$

we have

$$
\boldsymbol{\gamma}_{\psi, j}^{\mathrm{T}} \hat{\boldsymbol{f}}_{j}=\sum_{i=1}^{p} \gamma_{i j} \hat{f}_{i j}
$$


We use the multinomial theorem [45]

$$
\left(x_{1}+x_{2}+\cdots+x_{m}\right)^{k}=\sum_{k_{1}+k_{2}+\cdots+k_{m}=k}\left(\begin{array}{c}
k \\
k_{1}, k_{2}, \ldots, k_{m}
\end{array}\right) \prod_{t=1}^{m} x_{t}^{k_{t}}
$$

where

$$
\left(\begin{array}{c}
k \\
k_{1}, k_{2}, \ldots, k_{m}
\end{array}\right)=\frac{k !}{k_{1} ! k_{2} ! \ldots k_{m} !}
$$

to calculate $\left(\boldsymbol{\gamma}_{\psi, j}^{\mathrm{T}} \hat{\boldsymbol{f}}_{j}\right)^{2}$ and $\left(\boldsymbol{\gamma}_{\psi, j}^{\mathrm{T}} \hat{\boldsymbol{f}}_{j+1}\right)^{2}$.

In our case, $k=2$ and $m=p$, so we obtain

$$
\left(\sum_{i=1}^{p} \gamma_{i j} \hat{f}_{i j}\right)^{2}=\sum_{i=1}^{p}\left(\gamma_{j i} \hat{f}_{m, j}\right)^{2}+2 \sum_{m=1}^{p-1} \sum_{n=m+1}^{p}\left(\gamma_{j m} \hat{f}_{m j}\right)\left(\gamma_{j n} \hat{f}_{n j}\right) .
$$

With this result, (4.20) can be expressed as

$$
\begin{aligned}
& \boldsymbol{x}^{\mathrm{T}}\left(\mathbf{U}_{j}^{\mathrm{T}}\left(\boldsymbol{\Gamma}_{j}+2 \mathbf{R}_{j}\right) \mathbf{U}_{j}+\mathbf{U}_{j+1}^{\mathrm{T}}\left(\boldsymbol{\Gamma}_{j}+2 \mathbf{R}_{j}\right) \mathbf{U}_{j+1}\right) \boldsymbol{x}-2\left(\delta_{j} \gamma_{\psi, j}^{\mathrm{T}} \mathbf{U}_{j}+\omega_{j} \gamma_{\psi, j} \mathbf{U}_{j+1}\right) \boldsymbol{x}+\delta_{j}^{2}+\omega_{j}^{2}-1 \\
& <0 \quad j=1,3, \ldots, 2 n_{i}-1
\end{aligned}
$$

in terms of $\boldsymbol{x}$, with the definitons

$$
\begin{aligned}
\mathbf{R}_{i} & =\left[\begin{array}{c}
\mathbf{I}_{p-1} \\
\mathbf{0}_{1 \times p-1}
\end{array}\right]\left[\begin{array}{lll}
\gamma_{j, 1} & & \\
& \ddots & \\
& & \gamma_{j, p-1}
\end{array}\right]\left[\begin{array}{c}
\boldsymbol{\gamma}_{i}^{\mathrm{T}} \mathbf{E}_{1} \\
\vdots \\
\gamma_{i}^{\mathrm{T}} \mathbf{E}_{p-1}
\end{array}\right] \\
\mathbf{E}_{n} & =\left[\begin{array}{lll}
\boldsymbol{e}_{n+1} & \ldots & \boldsymbol{e}_{p}
\end{array}\right]\left[\begin{array}{c}
\boldsymbol{e}_{n+1}^{\mathrm{T}} \\
\vdots \\
\boldsymbol{e}_{n_{z}}^{\mathrm{T}}
\end{array}\right] \\
\boldsymbol{\Gamma}_{i} & =\left[\begin{array}{lll}
\gamma_{i 1}^{2} & \\
& \ddots & \gamma_{i p}^{2}
\end{array}\right] \\
\mathbf{U}_{i} & =\left[\begin{array}{lll}
\mathbf{0}_{p \times p(i-1)} & \mathbf{I}_{p} & \mathbf{0}_{p \times p\left(n_{z}-i\right)}
\end{array}\right]
\end{aligned}
$$

and where $\boldsymbol{e}_{i} \in \mathbb{R}^{p}$ denotes the $i$ th standard basis vector. As an example, for $p=2$ we would have

$$
\boldsymbol{e}_{1}=\left[\begin{array}{l}
1 \\
0
\end{array}\right] \quad \boldsymbol{e}_{2}=\left[\begin{array}{l}
0 \\
1
\end{array}\right]
$$


For better readability, we introduce

$$
\begin{aligned}
\mathbf{S}_{j} & :=\boldsymbol{\Gamma}_{j}+2 \mathbf{R}_{j} \\
\boldsymbol{\epsilon}_{j}^{\mathrm{T}} & =\delta_{j} \boldsymbol{\gamma}_{\psi, j}^{\mathrm{T}} \mathbf{U}_{j}+\omega_{j} \boldsymbol{\gamma}_{\psi, j} \mathbf{U}_{j+1} \\
r_{j} & =\delta_{j}^{2}+\omega_{j}^{2}-1
\end{aligned}
$$

so (A.19) becomes

$$
\boldsymbol{x}^{\mathrm{T}}\left(\mathbf{U}_{j}^{\mathrm{T}} \mathbf{S}_{j} \mathbf{U}_{j}+\mathbf{U}_{j+1}^{\mathrm{T}} \mathbf{S}_{j} \mathbf{U}_{j+1}\right) \boldsymbol{x}-2 \boldsymbol{\epsilon}_{j}^{\mathrm{T}} \boldsymbol{x}+r_{j}<0 \quad j=1,3, \ldots, 2 n_{i}-1
$$

Next, the objective function $\|\hat{\mathbf{F}} \mathbf{W}\|_{F}^{2}$ will be expressed in terms of $\boldsymbol{x}$. We have

$$
\|\hat{\mathbf{F}} \mathbf{W}\|_{F}^{2}=\sum_{i=1}^{p} \sum_{j=1}^{n_{c}}\left(\sum_{k=1}^{n_{z}} \hat{f}_{i k} w_{k j}\right)^{2}
$$

with

$$
\begin{aligned}
\hat{\mathbf{F}} & =\left[\begin{array}{cccccc}
\hat{f}_{11} & \ldots & \hat{f}_{1 n_{z}} & 0 & \ldots & 0 \\
\vdots & \ddots & \vdots & \vdots & \ddots & \vdots \\
\hat{f}_{p 1} & \ldots & \hat{f}_{p n_{z}} & 0 & \ldots & 0
\end{array}\right] \\
\mathbf{W} & =\left[\begin{array}{ccc}
w_{11} & \ldots & w_{1 n_{c}} \\
\vdots & \ddots & \vdots \\
w_{n_{c} 1} & \ldots & w_{n_{c} n_{c}}
\end{array}\right]
\end{aligned}
$$

and, when we use the multinomial theorem again, we get

$$
\|\hat{\mathbf{F}} \mathbf{W}\|_{F}^{2}=\boldsymbol{x}^{\mathrm{T}} \mathbf{Y} \boldsymbol{x}
$$


with

$$
\begin{aligned}
& \mathbf{Y}=\sum_{i=1}^{p} \sum_{j=1}^{n_{c}} \mathbf{R}_{i}^{\mathrm{T}} \mathbf{W}_{j} \mathbf{R}_{i}+\mathbf{J}_{i} \widetilde{\mathbf{W}}_{j} \mathbf{R}_{i} \\
& \mathbf{R}_{i}=\left[\begin{array}{c}
\boldsymbol{e}_{i}^{\mathrm{T}} \\
\boldsymbol{e}_{p+i}^{\mathrm{T}} \\
\vdots \\
\boldsymbol{e}_{\left(n_{z}-1\right) p+i}^{\mathrm{T}}
\end{array}\right] \quad \boldsymbol{e}_{i} \in \mathbb{R}^{p \cdot n_{z}} \\
& \mathbf{W}_{j}=\left[\begin{array}{ccc}
w_{1 j}^{2} & & \\
& \ddots & \\
& & w_{n_{z j}}^{2}
\end{array}\right] \\
& \mathbf{J}_{i}=2\left[\begin{array}{llll}
\boldsymbol{e}_{i} & \boldsymbol{e}_{p+1} & \ldots & \boldsymbol{e}_{\left(n_{z}-2\right) p+i}
\end{array}\right] \quad \boldsymbol{e}_{i} \in \mathbb{R}^{p \cdot n_{z}} \\
& \widetilde{\mathbf{W}}_{j}=\left[\begin{array}{lll}
w_{1 j} & & \\
& \ddots & \\
& & w_{\left(n_{z}-1\right) j}
\end{array}\right]\left[\begin{array}{c}
\widetilde{\boldsymbol{w}}_{j}^{\mathrm{T}} \mathbf{E}_{1} \\
\vdots \\
\widetilde{\boldsymbol{w}}_{j}^{\mathrm{T}} \mathbf{E}_{n_{z}-1}
\end{array}\right] \\
& \mathbf{E}_{n}=\left[\begin{array}{lll}
\boldsymbol{e}_{n+1} & \ldots & \boldsymbol{e}_{n_{z}}
\end{array}\right]\left[\begin{array}{c}
\boldsymbol{e}_{n+1}^{\mathrm{T}} \\
\vdots \\
\boldsymbol{e}_{n_{z}}^{\mathrm{T}}
\end{array}\right] \quad \boldsymbol{e}_{i} \in \mathbb{R}^{n_{z}} \\
& \widetilde{\boldsymbol{w}}_{j}^{\mathrm{T}}=\left[\begin{array}{lll}
w_{1 j} & \ldots & w_{n_{z} j}
\end{array}\right] .
\end{aligned}
$$

Together with the previous results we can write the optimization problem as:

$$
\begin{array}{ll}
\operatorname{minimize} & \boldsymbol{x}^{\mathrm{T}} \mathbf{Y} \boldsymbol{x} \\
\text { subject to } & \boldsymbol{x}^{\mathrm{T}}\left(\mathbf{U}_{j}^{\mathrm{T}} \mathbf{S}_{j} \mathbf{U}_{j}+\mathbf{U}_{j+1}^{\mathrm{T}} \mathbf{S}_{j} \mathbf{U}_{j+1}\right) \boldsymbol{x}-2 \boldsymbol{\epsilon}_{j}^{\mathrm{T}} \boldsymbol{x}+r_{j}<0 \quad i=1,3, \ldots, 2 n_{i}-1, \\
& \mathbf{M N} \boldsymbol{x}+\boldsymbol{k}<\mathbf{0} \\
& \mathbf{G} \boldsymbol{x}=\mathbf{0}
\end{array}
$$


where

$$
\mathbf{G}=\left[\begin{array}{c}
\boldsymbol{\Gamma}_{1} \mathbf{T}_{1} \\
\vdots \\
\boldsymbol{\Gamma}_{n_{i}} \mathbf{T}_{n_{i}} \\
\tilde{\boldsymbol{\Gamma}}_{1} \tilde{\mathbf{T}}_{1} \\
\vdots \\
\tilde{\boldsymbol{\Gamma}}_{n_{i}} \tilde{\mathbf{T}}_{n_{i}} \\
\hat{\boldsymbol{\Gamma}}_{1} \hat{\mathbf{T}}_{1} \\
\vdots \\
\hat{\boldsymbol{\Gamma}}_{n_{i}} \hat{\mathbf{T}}_{n_{i}}
\end{array}\right]
$$

was defined for better readability.

Next, we want to introduce the Lagrangian with parameters $\boldsymbol{\mu} \in \mathbb{R}^{2 n_{z}-3 n_{i}}$ and $\boldsymbol{\nu} \in \mathbb{R}^{n_{z}\left(n_{z}-1\right)}$ (we have $2 n_{z}-3 n_{i}$ inequality constraints and $n_{z}\left(n_{z}-1\right)$ equality constraints in (A.28)). Let $\boldsymbol{\mu}$ be partitioned as

$$
\begin{aligned}
\boldsymbol{\mu}_{1} & =\left[\begin{array}{lll}
\mu_{1} & \ldots & \mu_{n_{i}}
\end{array}\right]^{\mathrm{T}} \\
\boldsymbol{\mu}_{2} & =\left[\begin{array}{lll}
\mu_{n_{i}+1} & \ldots & \mu_{2 n_{z}-3 n_{i}}
\end{array}\right]^{\mathrm{T}} \\
\boldsymbol{\mu} & =\left[\begin{array}{l}
\boldsymbol{\mu}_{1} \\
\boldsymbol{\mu}_{2}
\end{array}\right]
\end{aligned}
$$

SO

$$
\begin{aligned}
L(\boldsymbol{x}, \boldsymbol{\mu}, \boldsymbol{\nu}) & =\boldsymbol{x}^{\mathrm{T}} \mathbf{Y} \boldsymbol{x}+\sum_{i=1}^{n_{i}} \mu_{i}\left(\boldsymbol{x}^{\mathrm{T}}\left(\mathbf{U}_{2 i-1}^{\mathrm{T}} \mathbf{S}_{2 i-1} \mathbf{U}_{2 i-1}+\mathbf{U}_{2 i}^{\mathrm{T}} \mathbf{S}_{2 i-1} \mathbf{U}_{2 i}\right) \boldsymbol{x}-2 \boldsymbol{\epsilon}_{2 i-1}^{\mathrm{T}} \boldsymbol{x}+r_{2 i-1}\right) \\
& +\boldsymbol{\mu}_{2}^{\mathrm{T}}(\mathbf{M N} \boldsymbol{x}+\boldsymbol{k})+\boldsymbol{\nu}^{\mathrm{T}} \mathbf{G} \boldsymbol{x}
\end{aligned}
$$

follows for the Lagrangian. We take a closer look at the sum:

$$
\begin{aligned}
& \sum_{i=1}^{n_{i}} \mu_{i}\left(\boldsymbol{x}^{\mathrm{T}}\left(\mathbf{U}_{2 i-1}^{\mathrm{T}} \mathbf{S}_{2 i-1} \mathbf{U}_{2 i-1}+\mathbf{U}_{2 i}^{\mathrm{T}} \mathbf{S}_{2 i-1} \mathbf{U}_{2 i}\right) \boldsymbol{x}-2 \boldsymbol{\epsilon}_{2 i-1}^{\mathrm{T}} \boldsymbol{x}+r_{2 i-1}\right) \\
& =\boldsymbol{x}^{\mathrm{T}}\left(\sum_{i=1}^{n_{i}} \mu_{i}\left(\mathbf{U}_{2 i-1}^{\mathrm{T}} \mathbf{S}_{2 i-1} \mathbf{U}_{2 i-1}+\mathbf{U}_{2 i}^{\mathrm{T}} \mathbf{S}_{2 i-1} \mathbf{U}_{2 i}\right)\right) \boldsymbol{x}-2\left(\sum_{i=1}^{n_{i}} \mu_{i} \boldsymbol{\epsilon}_{2 i-1}^{\mathrm{T}}\right) \boldsymbol{x}+\sum_{i=1}^{n_{i}} \mu_{i} r_{2 i-1} .
\end{aligned}
$$


With the definitions

$$
\begin{aligned}
\mathbf{H}\left(\boldsymbol{\mu}_{1}\right)= & \sum_{i=1}^{n_{i}} \mu_{i}\left(\mathbf{U}_{2 i-1}^{\mathrm{T}} \mathbf{S}_{2 i-1} \mathbf{U}_{2 i-1}+\mathbf{U}_{2 i}^{\mathrm{T}} \mathbf{S}_{2 i-1} \mathbf{U}_{2 i}\right) \\
\mathcal{E}= & {\left[\begin{array}{c}
\boldsymbol{\epsilon}_{1}^{\mathrm{T}} \\
\boldsymbol{\epsilon}_{3}^{\mathrm{T}} \\
\vdots \\
\boldsymbol{\epsilon}_{2 n_{i}-1}^{\mathrm{T}}
\end{array}\right] } \\
\boldsymbol{r}= & {\left[\begin{array}{c}
r_{1} \\
r_{3} \\
\vdots \\
r_{2 n_{i}-1}
\end{array}\right] }
\end{aligned}
$$

we can simplify the sum to

$$
\begin{aligned}
& \boldsymbol{x}^{\mathrm{T}}\left(\sum_{i=1}^{n_{i}} \mu_{i}\left(\mathbf{U}_{2 i-1}^{\mathrm{T}} \mathbf{S}_{2 i-1} \mathbf{U}_{2 i-1}+\mathbf{U}_{2 i}^{\mathrm{T}} \mathbf{S}_{2 i-1} \mathbf{U}_{2 i}\right)\right) \boldsymbol{x}-2\left(\sum_{i=1}^{n_{i}} \mu_{i} \boldsymbol{\epsilon}_{2 i-1}^{\mathrm{T}}\right) \boldsymbol{x}+\sum_{i=1}^{n_{i}} \mu_{i} r_{2 i-1} \\
& =\boldsymbol{x}^{\mathrm{T}} \mathbf{H} \boldsymbol{x}-2 \boldsymbol{\mu}_{1}^{\mathrm{T}} \boldsymbol{\mathcal { E }} \boldsymbol{x}+\boldsymbol{\mu}_{1}^{\mathrm{T}} \boldsymbol{r} .
\end{aligned}
$$

Please note that $\mathbf{H}$ depends on the first $n_{i}$ entries of the Lagrangian multiplier $\boldsymbol{\mu}$. This result yields

$$
L(\boldsymbol{x}, \boldsymbol{\mu}, \boldsymbol{\nu})=\boldsymbol{x}^{\mathrm{T}} \mathbf{Y} \boldsymbol{x}+\boldsymbol{x}^{\mathrm{T}} \mathbf{H} \boldsymbol{x}-2 \boldsymbol{\mu}_{1}^{\mathrm{T}} \mathcal{E} \boldsymbol{x}+\boldsymbol{\mu}_{1}^{\mathrm{T}} \boldsymbol{r}+\boldsymbol{\mu}_{2}^{\mathrm{T}}(\mathbf{M N} \boldsymbol{x}+\boldsymbol{k})+\boldsymbol{\nu}^{\mathrm{T}} \mathbf{G} \boldsymbol{x}
$$

for the Lagrangian, after we plugged these definitions back into (A.31).

Additionally, we define

$$
\begin{aligned}
& \mathbf{Q}=\mathbf{Y}+\mathbf{H} \\
& \mathbf{P}=\left[\begin{array}{l}
-2 \mathcal{E} \\
\mathbf{M N}
\end{array}\right] \\
& \boldsymbol{d}=\left[\begin{array}{l}
\boldsymbol{r} \\
\boldsymbol{k}
\end{array}\right]
\end{aligned}
$$

so we finally obtain

$$
L(\boldsymbol{x}, \boldsymbol{\mu}, \boldsymbol{\nu})=\boldsymbol{x}^{\mathrm{T}} \mathbf{Q} \boldsymbol{x}+\left(\boldsymbol{\mu}^{\mathrm{T}} \mathbf{P}+\boldsymbol{\nu}^{\mathrm{T}} \mathbf{G}\right) \boldsymbol{x}+\boldsymbol{\mu}^{\mathrm{T}} \boldsymbol{d}
$$

as the Lagrangian. 
As a next step, we want to examine the matrix Q. It is defined as the sum of two matrices, $\mathbf{Y}$ and $\mathbf{H}$. It can be shown that $\mathbf{Y}$ is an upper triangular matrix where the main diagonal entries consist of the terms

$$
\sum_{j=1}^{n_{c}} w_{1 j}^{2} \quad \sum_{j=1}^{n_{c}} w_{2 j}^{2} \quad \ldots \quad \sum_{j=1}^{n_{z}} w_{n_{z} j}^{2} .
$$

For each $i=1,2, \ldots, n_{z}$, the sum $\sum_{j=1}^{n_{c}} w_{i j}^{2}$ appears $p$ times on the main diagonal, so $\mathbf{Y}$ has the form $(*$ denotes an element that could be $\neq 0$ )

$$
\mathbf{Y}=\left[\begin{array}{cccc}
\sum_{j=1}^{n_{c}} w_{1 j}^{2} & * & \ldots & * \\
0 & \ddots & \ddots & \vdots \\
\vdots & \ddots & \ddots & * \\
0 & \cdots & 0 & \sum_{j=1}^{n_{c}} w_{n_{z} j}^{2}
\end{array}\right]
$$

Because $\mathbf{W}$ is the inverse of the transformation matrix $\mathbf{V}$ which transformed $\widetilde{\boldsymbol{\Phi}}_{f}$ to modal canonical form, we know that $\mathbf{W}$ has full rank and therefore does not posses a row full of zeros. Thus we can conclude that $\mathbf{Y}$ is an upper triangular matrix with main diagonal entries $>0$, so $\mathbf{Y}$ is positive definite.

Moreover, it can be shown that

$$
\begin{aligned}
\mathbf{H} & =\left[\begin{array}{cccc}
\mu_{1} \tilde{\mathbf{S}}_{1} & & & \\
& \ddots & & \tilde{\mathbf{S}}_{2 n_{i}-1} \\
& & \mathbf{0}_{p\left(n_{z}-2 n_{i}\right) \times p\left(n_{z}-2 n_{i}\right)}
\end{array}\right] \\
\tilde{\mathbf{S}}_{j} & =\left[\begin{array}{llll}
\mathbf{S}_{j} & \\
& \mathbf{S}_{j}
\end{array}\right] \\
\mathbf{S}_{j} & =\left[\begin{array}{cccc}
\gamma_{j 1}^{2} & * & \ldots & * \\
0 & \ddots & \ddots & \vdots \\
\vdots & \ddots & \ddots & * \\
0 & \cdots & 0 & \gamma_{j p}^{2}
\end{array}\right]
\end{aligned}
$$

holds, so $\mathbf{S}_{j}$ is an upper triangular matrix with elements $\geq 0$ on the main diagonal. As a consequence, $\mathbf{H}$ is an upper triangular matrix with elements $\geq 0$ on the main diagonal as well. This leads to the result that $\mathbf{Q}=\mathbf{Y}+\mathbf{H}$ is an upper triangular matrix with elements $>0$ on the main diagonal, so it is positive definite. 


\section{BIBLIOGRAPHY}

Abramovitch, D. Y., Andersson, S. B., Pao, L. Y., and Schitter, G., "A tutorial on the mechanisms, dynamics, and control of atomic force microscopes," in 2007 American Control Conference, 2007, pp. 3488-3502.

Aphale, S. S., Devasia, S., and Moheimani, S. O. R., "Achieving high-bandwidth nanopositioning in presence of plant uncertainties," in 2008 IEEE/ASME International Conference on Advanced Intelligent Mechatronics, 2008, pp. 943948.

Butterworth, J. A., Pao, L. Y., and Abramovitch, D. Y., "The effect of nonminimum-phase zero locations on the performance of feedforward modelinverse control techniques in discrete-time systems," in 2008 American Control Conference, 2008, pp. 2696-2702.

Butterworth, J. A., Pao, L. Y., and Abramovitch, D. Y., "The effect of nonminimum-phase zero locations on the performance of feedforward modelinverse control techniques in discrete-time systems," in 2008 American Control Conference, 2008, pp. 2696-2702.

Dai, L., Li, X., Zhu, Y., and Zhang, M., "Quantitative analysis on tracking error under different control architectures and feedforward methods," in 2019 American Control Conference (ACC), 2019, pp. 5680-5686.

Devasia, S., "Output tracking with nonhyperbolic and near nonhyperbolic internal dynamics: Helicopter hover control," Journal of Guidance, Control, and Dynamics, vol. 20, no. 3, pp. 573-580, 1997. [Online]. Available: https://doi.org/10.2514/2.4079

Fujimoto, H., Hori, Y., and Kawamura, A., "Perfect tracking control based on multirate feedforward control with generalized sampling periods," IEEE Transactions on Industrial Electronics, vol. 48, no. 3, pp. 636-644, 2001.

Konigorski, U., "Entwurf robuster strukturbeschränkter Zustandsregelungen durch Polgebietsvorgabe mittels Straffunktionen / Design of robust structurally-constrained state feedback controllers by pole region assignment using penalty functions," at - Automatisierungstechnik, vol. 35, no. 1-12, pp. 254 - 258, 1987. [Online]. Available: https://www.degruyter.com/view/ journals/auto/35/1-12/article-p254.xml

Leang, K., Zou, Q., and Devasia, S., "Feedforward control of piezoactuators in atomic force microscope systems," IEEE Control Systems, vol. 29, pp. 70-82, 2009. 
Marro, G., Prattichizzo, D., and Zattoni, E., "Convolution profiles for right inversion of multivariable non-minimum phase discrete-time systems," Automatica, vol. 38, pp. 1695-1703, 102002.

Michałek, M. M., "Simple causal fixed-structure feedforward control law for general continuous-time lti siso systems," in 2014 European Control Conference (ECC), 2014, pp. 61-66.

Oppenheimer, M. W. and Doman, D. B., "Control of an unstable, nonminimum phase hypersonic vehicle model," in 2006 IEEE Aerospace Conference, 2006, pp. 7 pp.-.

Pao, L. Y., Butterworth, J. A., and Abramovitch, D. Y., "Combined feedforward/feedback control of atomic force microscopes," in 2007 American Control Conference, 2007, pp. 3509-3515.

Potsaid, B. and Wen, J. T., "High performance motion tracking control," in Proceedings of the 2004 IEEE International Conference on Control Applications, 2004., vol. 1, 2004, pp. 718-723 Vol.1.

Qingze Zou and Devasia, S., "Preview-based optimal inversion for output tracking: application to scanning tunneling microscopy," IEEE Transactions on Control Systems Technology, vol. 12, no. 3, pp. 375-386, 2004.

Rigney, B. P., Pao, L. Y., and Lawrence, D. A., "Nonminimum phase dynamic inversion for settle time applications," IEEE Transactions on Control Systems Technology, vol. 17, no. 5, pp. 989-1005, 2009.

Shiraishi, T. and Fujimoto, H., "Trajectory tracking control method based on zero-phase minimum-phase factorization for nonminimum-phase continuoustime system," in 2012 12th IEEE International Workshop on Advanced Motion Control (AMC), 2012, pp. 1-6.

Åström, K., Hagander, P., and Sternby, J., "Zeros of sampled systems," Automatica, vol. 20, no. 1, pp. 31 - 38, 1984. [Online]. Available: http://www.sciencedirect.com/science/article/pii/0005109884900621

Tomizuka, M., "Zero Phase Error Tracking Algorithm for Digital Control," Journal of Dynamic Systems, Measurement, and Control, vol. 109, no. 1, pp. 65-68, 03 1987. [Online]. Available: https://doi.org/10.1115/1.3143822

Tsao, T.-C. and Tomizuka, M., "Adaptive Zero Phase Error Tracking Algorithm for Digital Control," Journal of Dynamic Systems, Measurement, and Control, vol. 109, no. 4, pp. 349-354, 12 1987. [Online]. Available: https://doi.org/10.1115/1.3143866 
Vaccaro, R. J., "An optimization approach to the pole-placement design of robust linear multivariable control systems," in 2014 American Control Conference, 2014, pp. 4298-4305.

Vaccaro, R. J., Digital Control: A State-Space Approach. McGraw-Hill, 1995.

Wen, J. T. and Potsaid, B., "An experimental study of a high performance motion control system," in Proceedings of the 2004 American Control Conference, vol. 6, 2004, pp. 5158-5163.

Ying Wu and Qingze Zou, "Robust-inversion-based 2dof-control design for output tracking: Piezoelectric actuator example," in 2007 46th IEEE Conference on Decision and Control, 2007, pp. 2451-2457.

Zou, Q. and Devasia, S., "Preview-based stable-inversion for output tracking," in Proceedings of the 1999 American Control Conference (Cat. No. 99CH36251), vol. 5, 1999, pp. 3544-3548 vol.5. 